\title{
Investigation of myelin maintenance and turnover by inducible MBP knock-out in adult mice
}

\author{
Dissertation \\ for the award of the degree \\ "Doctor rerum naturalium" (Dr.rer.nat.) \\ of the Georg August University Göttingen \\ within the doctoral program \\ Molecular Physiology of the Brain \\ of the Georg-August University School of Science (GAUSS)
}

Submitted by

Martin Meschkat

From

Witten, Germany

Göttingen,

25.04 .2019 


\section{Examination Board}

\section{Thesis Committee}

Dr. Wiebke Möbius (1. Referee)

Electron Microscopy Core Unit

Max Planck Institute of Experimental Medicine

Prof. Dr. Dr. Hannelore Ehrenreich (2. Referee)

Research Group Clinical Neuroscience

Max Planck Institute of Experimental Medicine

Dr. Katrin Willig

Research Group Optical Nanoscopy in Neuroscience

University Medical Center Göttingen

\section{Extended Examination Board}

Prof. Dr. Christine Stadelmann-Nessler

Institut für Neuropathologie, Klinik für Neurologie

University Medical Center Göttingen

Prof. Dr. Thomas Bayer

Abteilung für Molekulare Psychiatrie, Klinik für Psychiatrie

University Medical Center Göttingen

Prof. Dr. Susann Boretius

Functional Imaging Laboratory

German Primate Center

Date of oral examination: 13.06.2019 


\section{Affidavit}

I hereby declare that the PhD thesis entitled "Investigation of myelin maintenance and turnover by inducible MBP knock-out in adult mice" has been written independently by myself with no other contributory sources and aids than quoted.

Göttingen, 25th April 2019

Martin Meschkat 


\section{Acknowledgements}

First of all, I would like to thank my supervisor Dr. Wiebke Möbius for intensive discussion and a lot of support. Her door was always open and she gave me the freedom to follow my own ideas. From her I learned new ways to visualize my science for which I am very grateful.

I would like to thank Prof. Klaus-Armin Nave for his support, scientific discussions and giving me the opportunity to conduct my research in his laboratory.

I would like to express my gratitude to my thesis committee members Prof. Dr. Hannelore Ehrenreich and Dr Katrin Willig for their interest in my project and discussion during the thesis committee meetings. I would also like to mention Prof Michael Hörner. He was a source of optimism and encouragement during my first two thesis committee meetings and will be missed. I also thank Prof. Dr. Christine Stadelmann, Prof. Dr. Thomas Bayer and Prof. Dr.Susann Boretius for being part of my examination board.

I thank my collaborators: Dr. Olaf Jahn and Lars Piepkorn for a large and complex proteome dataset, intensive discussion and ongoing support with its analysis. I would like to thank Prof. Dr. Britta Brügger and her team for the lipidome dataset.

I am very grateful for the enormous support I got from the team of the Electron microscopy core unit. Boguscha, Torben, Christos and Anna were helping along the whole project and we had many joyful moments. I would like to thank Boguscha and Torben for all their patience and support in the lab. I learned many things from them and they were involved in all EM experiments. I would like to thank Anna Steyer for FIB measurements and training. A big thanks to Christos Nardis for his helpful sarcasm and a lot of fruitful discussion about 3D image analysis and quantification.

I am very very grateful for all the awesome technical support by Ramona Jung, Ulli Bode, Anette Fahrenholz and Ursula Kutzke. They made my life so much easier and helped me countless times.

I would also like to thank the teamin the animal house especially Cornelia Casper, Sarah Schulze and Tanja Hoffmeister who did an great job in taking care of our mice.

My thank also goes to Dr. Marie-Theres Weil for an great time in the lab and fascinating discussion. I am also thankful for scientific and personal discussion with Dr. Kathrin Kusch, Dr. Theresa Kungel, Iva Tzvetanova PhD. and Dr. Hauke Werner.

I would like to thank my colleagues and friends (in alphabetical order) Andrea Trevisiol, Jan Seidel, Lena Spieth, Maria Eichel, Niklas Michel, Ramona Jung, Sophie Siems, Stefan Berghoff, Tim Düking, Tobias Buscham, Ulrich Weikert, Jan Winchenbach and all the other people in the Nave lab and the MPI EM. I really enjoyed the long evenings in the lab, the cooking events, the Phd sports, the very cool retreats and am thankful for all the help and support over these years.

Especially I would to thank Ramona and Tim for a lot of emotional support, for all the coffee breaks without coffee, for the laughter and positivity and for just being there.

I thank Michaela Schmalstieg and Gabriele Endo for help with administrative issues.

For IT support I am grateful to Hajo Horn, Rolf Merker and Lothar Demel. 
The funding for this $\mathrm{PhD}$ research was provided by the Cluster of Excellence Nanoscale Microscopy and Molecular Physiology of the Brain (CNMPB) and the department of Neurogenetics for which I am grateful.

I would like to highlight how much I appreciate the invaluable help and support of my friends outside of the institute. My closest friend Max who helped me sort my thoughts and supported me for all these years. Also, I would like to mention all the close Göttinger friends Julia, Felix, Jule, Philipp, Tim, Evi, Hannes, Max and Moritz for their friendship and support.

All this would not have been possible without the constant support of my parents Beate and Reinhard Meschkat and my brother Timo. They motivated me to always follow my dreams and do what I considered to be right. Thank you for all your love, the guidance and that you believe in me. 
Für meine Familie 
The most exciting phrase to hear in science, the one that heralds new discoveries, is not 'Eureka', but 'That's funny...'

Isaac Asimov 


\section{Abstract}

\section{The turnover and maintenance of myelin in the adult CNS}

Myelin is a multilayered membrane structure generated by oligodendrocytes in the CNS that insulates and supports axons. While most of the oligodendrocyte population differentiates early in life and persist for most of the lifetime, myelin proteins are slowly turned over and replaced within weeks to months. However, the mechanisms of this turnover and replacement processes within the myelin sheath are largely unknown. One reason is the absence of a suitable model system. To study myelin turnover in the adult animal a model system is required that allows to investigate myelin maintenance under conditions of ongoing turnover, survival of oligodendrocytes and the avoidance of acute inflammation. In this thesis a new model system was established that meets these requirements and was used to study turnover within the individual myelin sheath. This model system is based on a strategy for the visualization of myelin renewal by the application of an inducible deletion of myelin basic protein ( $M b p$ ) expression in the adult. For this purpose, ablation of MBP was induced in young adult mice after completion of developmental myelination at the age of 8 weeks. After the knock-out was induced, any newly synthetized myelin membrane lacked MBP and therefore the essential component for myelin compaction. The absence of compaction served as a marker for newly made myelin and was studied by electron microscopy. Using volume imaging by focused ion beam scanning electron microscopy (FIBSEM) we observed the emergence of non-compact myelin membranes at the inner tongue of the juxta-paranodal region. The localized emergence of these membranes suggests the juxta-paranode as a site of MBP integration within the myelin sheath. Furthermore, the progressive loss of compact myelin after ablation of MBP synthesis was quantified and a shortening of internodes in the optic nerve by 50\% within 19-21 weeks was observed. This shows that under these conditions half of the internode is turned over within 4-5 months. We performed proteome analysis of the optic nerve eight, 16 and 40 weeks after Mbp knockout induction to investigate systemic responses after loss of de novo MBP synthesis and found reduced abundance for proteins associated to the myelin sheath and cell adhesion molecules while many cytoskeleton-associated proteins were increased in abundance. These results are in accordance with the detected loss of the compact myelin compartment. To identify potential mechanisms for oligodendrocyte intrinsic or extrinsic recycling of myelin we analyzed individual axons in 3D FIB-SEM volumes and detected an increased number of myelin spheres at the inner tongue of the oligodendrocytes. We suggest that these myelin spheres are very likely involved in disposal of aged myelin. Visualization of the observed processes of membrane integration and removal were made possible by combination of high-quality structural preservation, 3D FIB-SEM microscopy and the novel mouse model system. In conclusion, this study introduced and characterized a powerful tool to study myelin maintenance and turnover and used this to shed light on the process of myelin turnover and the half-life of a myelin sheath in CNS of adult mice. 


\section{List of abbreviations:}

\begin{tabular}{|c|c|}
\hline A & Ampere \\
\hline Aa & Amino acid \\
\hline APS & Ammonium persulfate \\
\hline Bp & Base pair \\
\hline BSA & Bovine serum albumin \\
\hline${ }^{\circ} \mathrm{C}$ & Degree celsius \\
\hline $\mathrm{CC}$ & Corpus Callosum \\
\hline $\mathrm{rCC}$ & Costal Corpus Callosum \\
\hline $\mathrm{cCC}$ & Caudal Corpus Callosum \\
\hline Ctx & Cortex \\
\hline Chol & Cholesterol \\
\hline Cit & Citrulline \\
\hline CNP & 2',3'-cyclic nucleotide 3'-phosphodiesterase \\
\hline CNS & Central nervous system \\
\hline d & Day \\
\hline DAPI & 4'-6'-Diamidino-2-phenylindole \\
\hline $\mathrm{ddH} 2 \mathrm{O}$ & deionized milli q-water comparable to double distilled water \\
\hline DMEM & Dulbecco's modified eagle's medium \\
\hline DMSO & Dimethylsulfoxide \\
\hline DNA & Deoxyribonucleic acid \\
\hline dNTP & Deoxynucleotide triphosphate \\
\hline EDTA & Ethylenediaminetetraacetic acid \\
\hline EM & Electron microscopy \\
\hline Epon & Epoxy resin \\
\hline FIB-SEM & Focussed Ion beam scanning electron microscopy \\
\hline g & Gram \\
\hline Golli & Genes of the oligodendrocyte lineage \\
\hline GS & Goat serum \\
\hline $\mathrm{h}$ & Hour \\
\hline HBSS & Hank's buffered saline \\
\hline IHC & Immunohistochemistry \\
\hline ip & Intraperitoneal \\
\hline $\mathrm{kDa}$ & Kilodalton \\
\hline $\mathrm{m}$ & Meter \\
\hline M & Molar \\
\hline MAG & Myelin associated glycoprotein \\
\hline MBP & Myelin basic protein \\
\hline $\min$ & Minute \\
\hline MOG & Myelin oligodendrocyte glycoprotein \\
\hline MS & Multiple Sclerosis \\
\hline n.s. & Non-significant \\
\hline n.opt & Optic nerve \\
\hline OsO4 & Osmiumtetroxyde \\
\hline $\mathrm{PB}$ & Phosphate buffer \\
\hline PBS & Phosphate buffered saline \\
\hline PCR & Polymerase chain reaction \\
\hline PFA & Paraformaldeyhde \\
\hline
\end{tabular}




$\begin{array}{ll}\text { PIP2 } & \text { Phosphatidylinositol-4,5-bisphosphat } \\ \text { PIP3 } & \text { Phosphatidylinositol }(3,4,5) \text {-trisphosphate } \\ \text { PLP } & \text { Proteolipid protein } \\ \text { PNS } & \text { Peripheral nervous system } \\ \text { ROI } & \text { Region of interest } \\ \text { pti } & \text { post tamoxifen injection } \\ \text { PVDF } & \text { Polyvinylidene difluoride } \\ \text { rpm } & \text { Rotations per minute } \\ \text { SD } & \text { Standard deviation } \\ \text { SDS } & \text { Sodiumdodecylsulfate } \\ \text { SEM } & \text { Scanning electron microscopy } \\ \text { TBS } & \text { TRIS-buffered saline } \\ \text { TEMED } & \text { Tetramethylenediamine } \\ \text { V } & \text { Volt } \\ \text { W } & \text { Weeks } \\ \text { WB } & \text { Western blot } \\ \text { WT } & \text { Wild type } \\ \text { w/v } & \text { Weight per volume } \\ \text { v/v } & \text { Volume per volume }\end{array}$




\section{Contents}

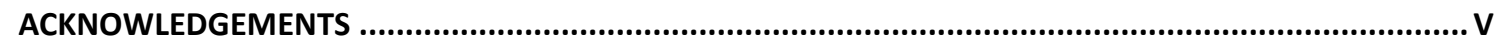

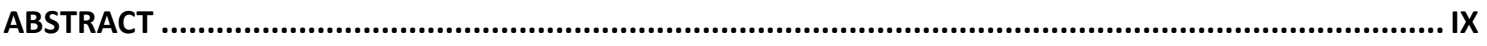

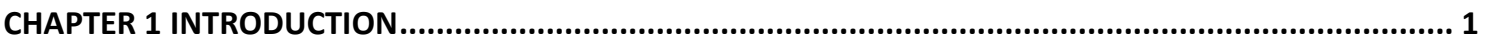

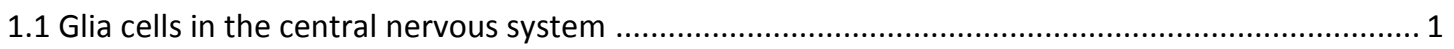

1.2 Myelin insulates axons and accelerates signal transduction ................................................... 1

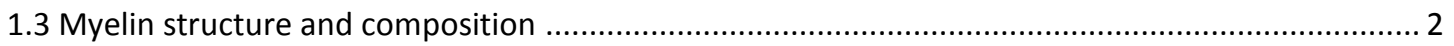

1.4 Myelin basic protein acts as molecular zipper of CNS myelin .................................................... 4

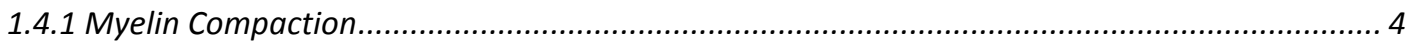

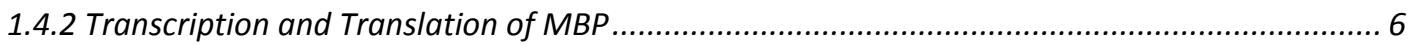

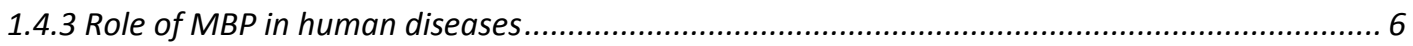

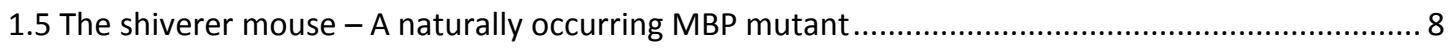

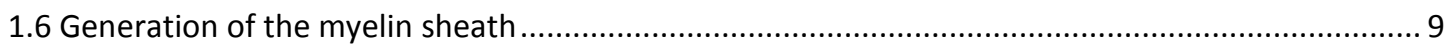

1.7 Myelin Turnover - Half-life of myelin components .............................................................. 12

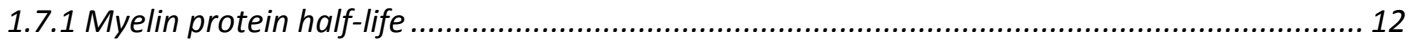

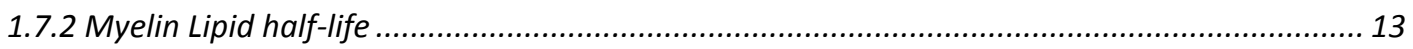

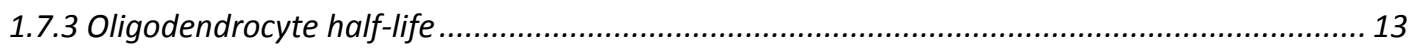

1.8 Myelin Degradation - Removal and recycling of myelin ........................................................ 15

1.9 Models for Demyelination - myelin turnover ......................................................................... 16

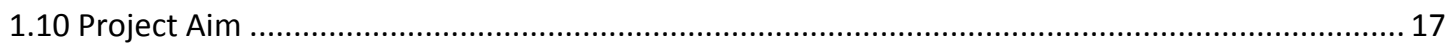

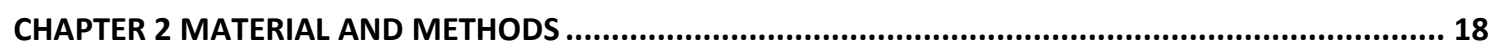

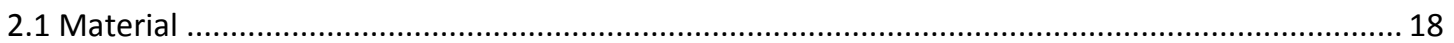

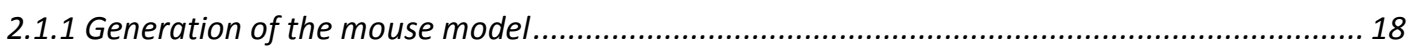

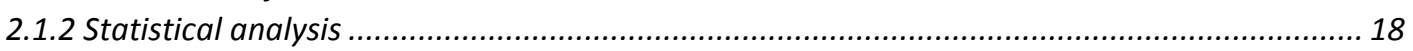

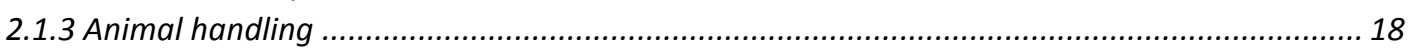

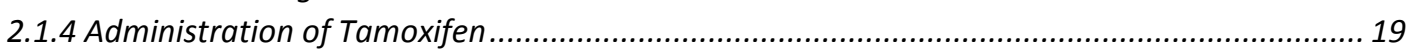

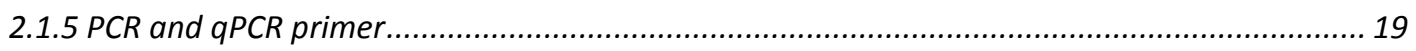

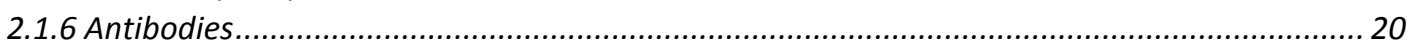

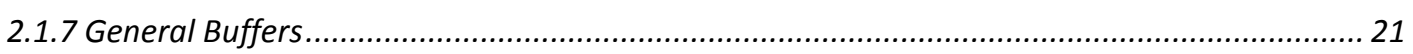

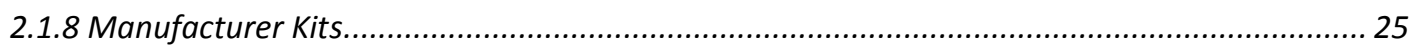

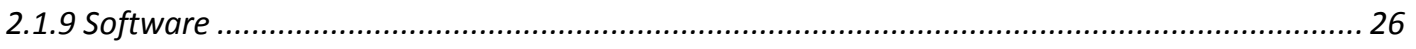

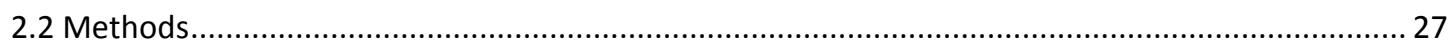

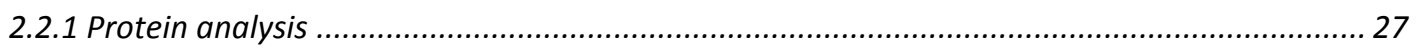

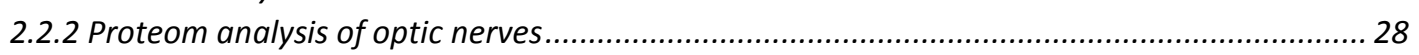

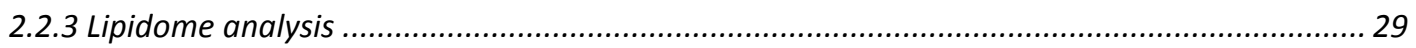

2.2.4 Isolation of oligodendrocytes by magnetic cell isolation (MACS) ....................................... 29

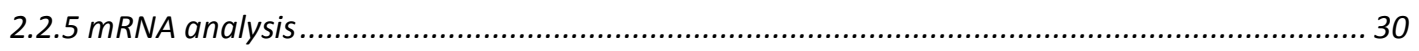

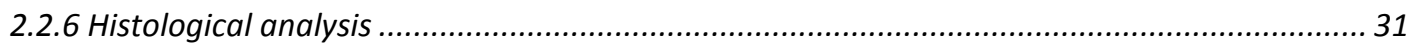

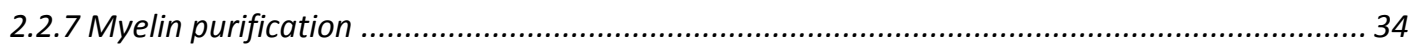

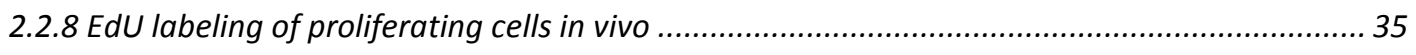

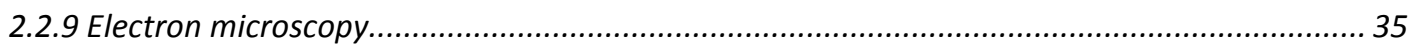

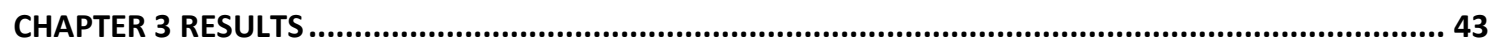

3.1 Generation of an inducible MBP knock-out mouse model.......................................................... 43

3.2 Decrease of Mbp mRNA after knock-out induction ............................................................... 44

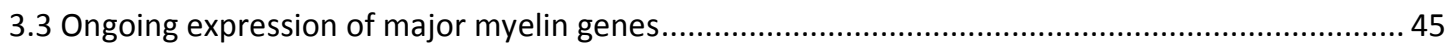

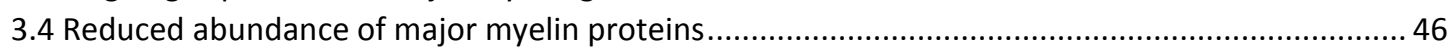

3.5 Tremor and motor impairments occur months after MBP ablation............................................ 49

3.6 Emergence and origin of non-compact membrane processes at the inner tongue ........................51 
3.7 Loss of myelinated profiles and decreased myelin thickness.....

3.8 No obvious ultrastructural changes in PNS and in the absence of tamoxifen ...............................60

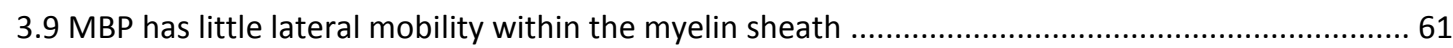

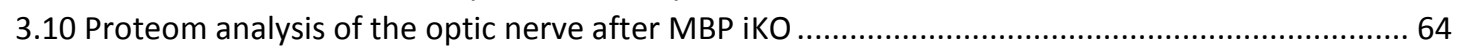

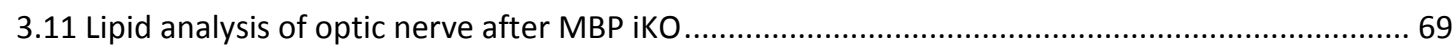

3.12 Microglial activation and astrogliosis upon MBP iKO ................................................................ 70

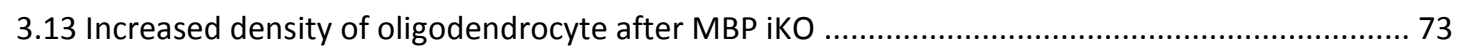

3.14 Cholesterol synthesis is affected on tissue level but not in oligodendrocytes............................. 75

3.15 Internodal shortening after MBP iKO .................................................................................... 78

3.16 Non-compact membrane tubules appear preferentially at the juxta-paranode ......................... 80

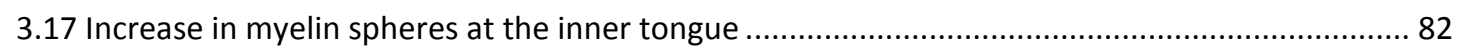

3.18 Signs of remyelination 52 weeks after knock-out induction .................................................... 87

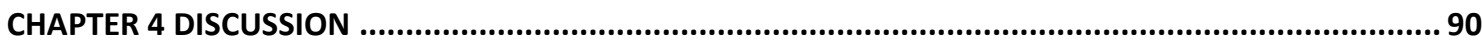

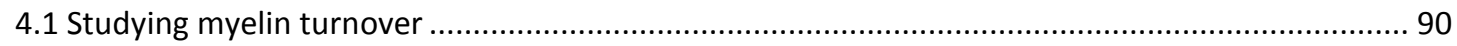

4.2 A new mouse model to study adult myelin turnover ........................................................... 91

4.3 Adult myelin turnover - Insertion of new membrane........................................................... 93

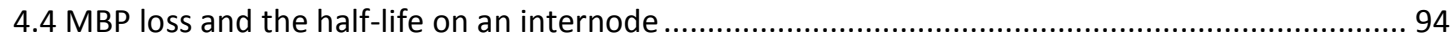

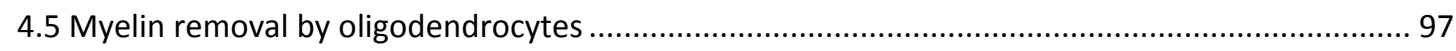

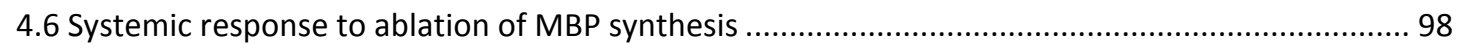

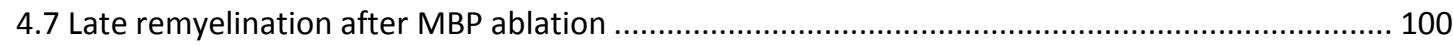

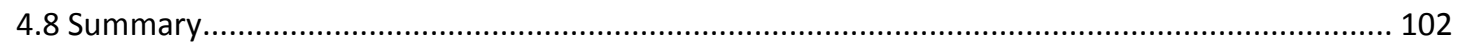

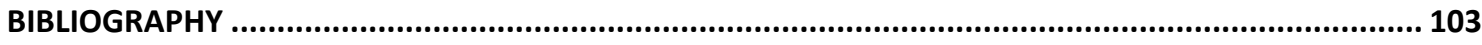

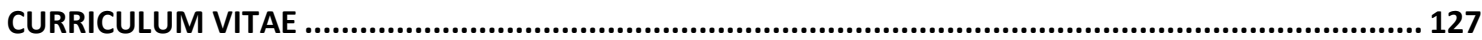




\section{Figures}

Figure 1-1: Cross section of an axon in the optic nerve. 3

Figure 1-2 Assembly of the major dense line upon formation of compact myelin ................................... 5

Figure 1-3 MBP is required for the formation of compact myelin ...................................................... 8

Figure 1-4 Process of myelination in the CNS.............................................................................. 10

Figure 1-5 Molecular organization of Adhesion proteins at the node of Ranvier ................................... 11

Figure 1-6 Microglial recycling of myelin fragments.................................................................... 15

Figure 3-1 Tamoxifen-inducible mouse model of Mbp ablation in adult mice ...................................... 43

Figure 3-2 MBP mRNA decreases within 20 days after first day of tamoxifen injections ......................... 44

Figure 3-3 The expression of PLP, Golli, MAG, MOG and CNP is not affected after MBP knock-out......... 45

Figure 3-4 Protein abundance of myelin proteins is decreases after MBP knock-out ............................ 47

Figure 3-5 Immunofluorescence staining for MBP in brain and optic nerve ......................................... 48

Figure 3-6 Tremor assessment and development of body weight after knockout induction .................... 50

Figure 3-7 Ablation of MBP results in formation of non-compact membrane tubules ............................. 51

Figure 3-8 Myelin tubulations are oligodendrocyte-derived membrane ............................................... 52

Figure 3-9 Membrane tubules occur predominantly at the inner tongue ..............................................53

Figure 3-10 Progressive loss of myelinated and increase in unmyelinated axons after ablation of MBP.. 56

Figure 3-11 Loss of compact myelin is observed in optic nerve, corpus callosum and spinal cord ............55

Figure 3-12 Increased corrected g-ratio in optic nerve ......................................................................5 58

Figure 3-13 Corrected g-ratio in spinal cord and rostral corpus callosum ............................................ 59

Figure 3-14 No obvious abnormalities in PNS myelin after MBP knock-out.............................................6 60

Figure 3-15 No myelin abnormalities in the absence of tamoxifen induced recombination .....................61

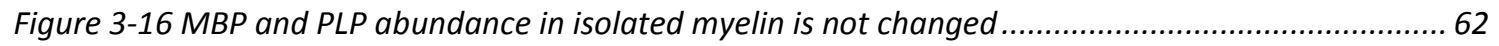

Figure 3-17 MBP has limited lateral mobility within the myelin sheath ................................................63

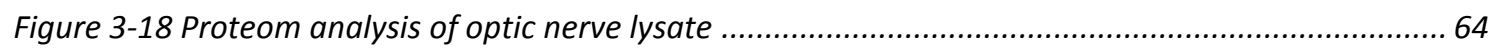

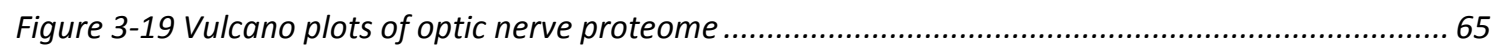

Figure 3-20 Relative protein composition shows reduction in major myelin proteins .............................6 66

Figure 3-21 Top 25 significantly increased and decreased proteins in optic nerve .................................6 67

Figure 3-22 Pathways enrichment of proteins with significantly increased abundance ........................... 68

Figure 3-23 Lipidome analysis after MBP iKO .............................................................................. 70

Figure 3-24 Progressive low grade astrogliosis, microgliosis and axonal pathology in Fimbria ................ 71

Figure 3-25 Progressive low grade astrogliosis, microgliosis and axonal pathology in the spinal cord..... 72

Figure 3-26 No significant increase in cell death after MBP iKO ........................................................ 73

Figure 3-27 Oligodendrocyte numbers are increased after MBP ablation ........................................... 75

Figure 3-28 Decreased cholesterol synthesis in corpus callosum $52 \mathrm{~W}$ pti ................................................. 76

Figure 3-29 Isolated $\mathrm{O4}^{+}$cells show no decrease in cholesterol synthesis genes ................................... 77

Figure 3-30 Internodal shortening after ablation of $M B P$................................................................. 80

Figure 3-31 Non-compact membrane tubules appear preferentially at the juxta-paranode.................... 82

Figure 3-32 Microglia and astrocytes take up myelin ......................................................................... 83

Figure 3-33 Thinning of compact myelin and increased number of myelin spheres at the inner tongue .. 85

Figure 3-34 Visualization of myelin spheres at the inner tongue ........................................................8 86

Figure 3-35 Remyelination in the optic nerve $52 \mathrm{~W}$ pti.......................................................................8 89

Figure 3-36 Increase in Mbp and Plp mRNA as signs of oligodendrocyte maturation and remyelination. 88

Figure 4-1 Indications for internodal shortening including paranode retraction .................................. 95

Figure 4-2 Presence of non-compact myelin tubules and myelin debris may inhibit remyelination ........ 101 


\section{Chapter 1 Introduction}

\subsection{Glia cells in the central nervous system}

The central nervous system (CNS) controls essential functions of the body and mind including the coordination of movement, regulating body functions such as heart rate, release of hormones and even more complex tasks like learning and memory. The mammalian central nervous system is composed of the brain and the spinal cord and is made up of a variety of cell types mainly divided into neurons and glia. While neurons generate, transmit and integrate information via changes in electrical potential, glial cells create the necessary framework and infrastructure for neurons to function. They are mainly divided in astrocytes, microglia and oligodendrocytes. Glial cells are essentially involved in all brain functions and are approximately as numerous as neurons in the human CNS (Allen and Barres, 2009; von Bartheld et al., 2016) with recent estimates suggesting that in human neocortex approximately $75 \%$ of glial cells are oligodendrocytes, $5 \%$ microglia and $20 \%$ are astrocytes (Pelvig et al., 2008; Butt and Verkhratsky, 2013) Astrocytes enclose endothelial cells, provide trophic support for other cell types, maintaining the homeostasis of water and ions in the brain (Kandel et al., 2012), contact and enclose synapses with their fine perisynaptic processes (Allen and Eroglu, 2017) and are involved in synaptic recycling (Allen and Barres, 2009). Microglia are the resident immune and inflammatory cells of the CNS and its primary defense against pathogens. They are involved in recycling of dead cells, pruning of synapses (Kandel et al., 2012) and the uptake of myelin (Safaiyan et al., 2016). A third branch of glial cells are the myelin forming oligodendrocytes. In vertebrates, the conduction of electrical signals along axons is accelerated through the formation of a multilamellar, lipid rich structure called myelin. The maintenance and turnover of myelin in the CNS of adult mice will be the main focus of this thesis.

\subsection{Myelin insulates axons and accelerates signal transduction}

Myelin, the insulating material of the nervous system, was first described by Rudolf Virchow in the early 19th century. He found myelin in tissue sections and described it as "nerve glue" that is providing stability and support for surrounding nerve cells (Virchow, 1854; Boullerne, 2016). Myelin evolved around 500 million years ago in cartilaginous fish as a new development of the vertebrate lineage (Zalc et al., 2008) and to date it is present in all living vertebrate species (Nawaz et al., 2013). Vertebrate myelin is a specialized multi-layered and lipid rich membrane that can be found in the peripheral nervous system where it is generated by Schwann cells and in the central nervous system where it is formed by oligodendrocytes. It provides several key advantages that might explain its success in the vertebrate lineage. The ensheathment of an axon by a multilayered myelin sheath allows a 10 - fold increase in conduction speed by saltatory signal propagation (Hartline and Colman, 2007). The insulation and increase in conduction speed allows for smaller axonal diameters (Baumann and Pham-Dinh, 2001), up to a 1000-fold lower energy consumption and higher signal fidelity compared to unmyelinated axons with similar conduction speed (Hartline and Colman, 2007; Wang et al., 2008). 
Oligodendrocytes, beside astrocytes, also provide metabolites for axonal energy production (Pellerin and Magistretti, 1994; Fünfschilling et al., 2012; Lee et al., 2012). The total length of an axon can be up to 1 meter and mitochondria occupy a relative constant percentage of $1.5 \%$ of axonal volume. This suggests a correlation between axonal diameter and energy requirements (Perge et al., 2009). The generation of large amounts of ATP in these mitochondria is required to maintain the axonal membrane potential by the activity of the $\mathrm{Na}+$ K+-ATPase (Almeida et al., 2001; Saez et al., 2014; Philips and Rothstein, 2017). Since neurons have high metabolic demand and low energy storage capacity in their long processes they are partly dependent on astrocytes and oligodendrocytes to generate the required amount of energy for restoration of the membrane potential (Philips and Rothstein, 2017). This observation is supported by experiments from Fünfschilling and colleagues who have suggested lactate as a possible metabolite in support of axons (Fünfschilling et al., 2012). The transfer of metabolites between oligodendrocyte and axon is mediated by the shuttling of lactate and pyruvate by monocarboxylate transporters located in the paranodal myelin (Saab et al., 2016). The provided lactate is then converted into pyruvate by neuronal lactate dehydrogenase and can enter the tricarboxylic acid (TCA) cycle to be used for axonal ATP synthesis (Fünfschilling et al., 2012; Lee et al., 2012; Saab et al., 2013). The lack of support by oligodendrocytes and a disturbed axon-glia unit are likely contributors to multiple sclerosis and amyotrophic lateral sclerosis where neurons are metabolically stressed emphasizing the importance of metabolic support for the maintenance of axonal health (Campbell and Mahad, 2014; Philips and Rothstein, 2017).

\subsection{Myelin structure and composition}

On ultrastructural level, as observed by electron microscopy, the myelin sheath (Figure 1-1) is a multilayered structure with alternating electron lucent and electron dense layers with a periodicity of $12 \mathrm{~nm}$ (Baumann and Pham-Dinh, 2001). The electron lucent layer is the intraperiod line formed by compaction of the outer myelin membrane layers by proteolipid protein (PLP). The darker layer is the major dense line that is formed by myelin basic protein (MBP) which compacts the cytosolic inner leaflets of the myelin membrane (Morell and Quarles, 1999). The thickness of the myelin sheath is dependent on the axonal diameter and can be described as the ratio between the axonal diameter divided by the diameter of the fiber including the myelin sheath. This parameter is called g-ratio and a range between 0.6 to 0.7 has been found to ensure optimal insulation (Rushton, 1951; Morell and Quarles, 1999; Hartline and Colman, 2007).

Myelin is divided into two compartments with specific molecular compositions: Noncompact and compact myelin. Non compact myelin contains cytoplasm and proteins like myelin-associated glycoprotein (MAG), or 2',3'-cyclic nucleotide 3'-phosphodiesterase (CNP), cytoskeletal elements like actin, tubulin or septins and organelles such as mitochondria, peroxisomes and vesicles (Aggarwal et al., 2011b; Patzig et al., 2016; Rinholm et al., 2016). The non-compact myelin compartment, namely the inner and outer tongue as well as cytoplasmic channels within the myelin sheath connect the oligodendrocyte to its myelin compartment (Nave and Werner, 2014; Snaidero et al., 2014). 


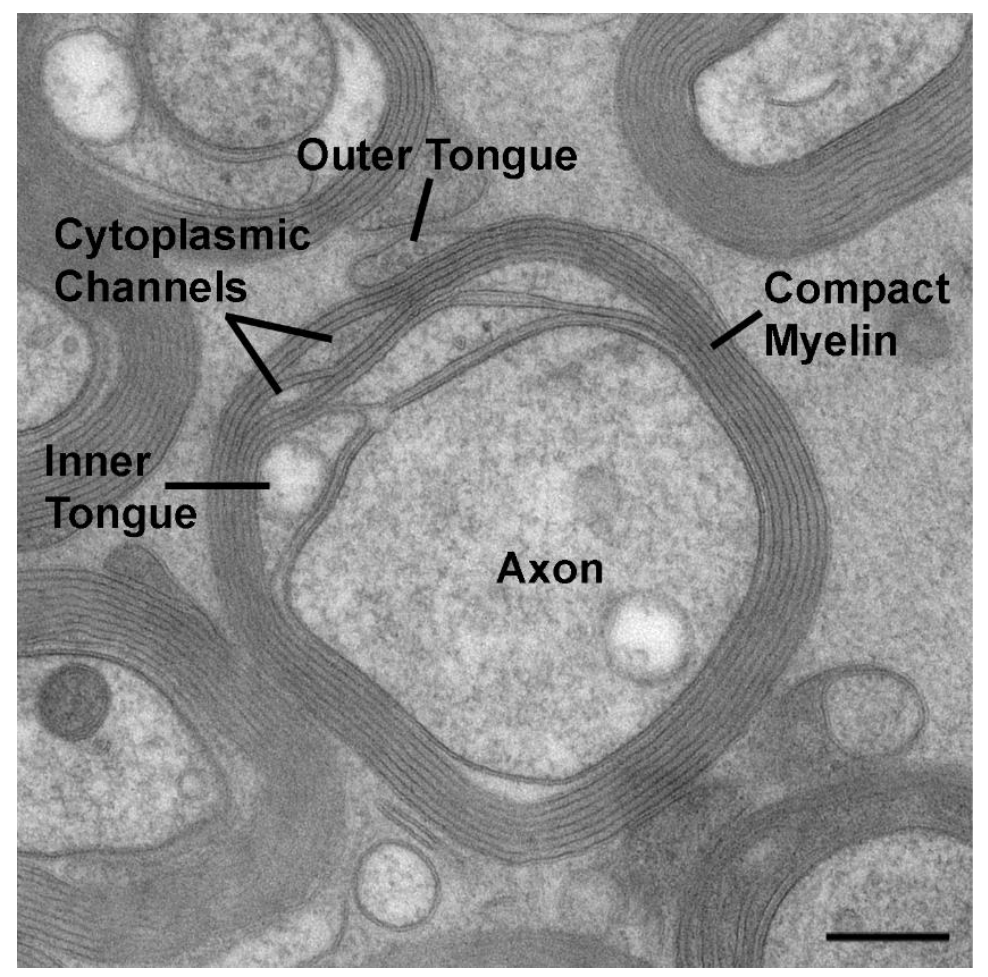

Figure 1-1: Cross section of an axon in the optic nerve

The retinal ganglion cell axon is wrapped by several layers of compact myelin with a dark major dense line and a lighter intraperiod line. The oligodendrocyte is in contact with its myelin compartment via non-compacted myelin areas such as the inner and outer tongue and the cytoplasmic channels. TEM Micrograph, High pressure frozen and freeze substituted optic nerve, scale bar $200 \mathrm{~nm}$.

Myelin membrane is composed of $70-80 \%$ lipids and $20-30 \%$ proteins having a high lipid content compared to other plasma membranes which mostly have equal ratio of lipids and proteins (Norton and Autili, 1965; Pfeiffer et al., 1993). The most abundant lipids in rodent myelin are cholesterol (27\%), galactosylcerebroside (GalC) (24\%) and ethanolaminecontaining plasmalogens (14\%) with GalC being the most typical myelin lipid as its amount is directly proportional to the amount of myelin in the brain (Morell and Quarles, 1999). Ablation of the synthesis of certain lipid classes is influencing myelination as for example the biosynthesis of cholesterol which is rate limiting for myelin biogenesis (Saher et al., 2005,2009 ). In contrast GalC synthesis by UDP glycosyltransferase 8 (ugt8a) is not required for the formation of myelin membrane but the formation of the node of Ranvier. In the absence of GalC, mice have normal appearing compact myelin but develop hindlimb paralysis and extensive myelin vacuolation suggesting that GalC might have a role in myelin maintenance (Coetzee et al., 1996; Dupree et al., 1998).

Major proteins of the myelin sheath include proteolipid protein (PLP), myelin oligodendrocyte glycoprotein (MOG), myelin-associated glycoprotein (MAG), 2',3'-cyclic nucleotide 3'-phosphodiesterase (CNP) and myelin basic protein (MBP).

Proteolipid protein (PLP), the most abundant myelin protein (17\%) (Jahn et al., 2009), belongs to the tetraspanin protein family and stabilizes the intraperiod line in compact myelin. Mice with ablated PLP have a compact myelin sheath with a condensed intraperiod line but develop axonal swellings and degeneration within the first year of life (Griffiths et al., 1998; Lüders et al., 2017). The ablation of PLP in adult animals after completion of myelination results in axonal pathology (Griffiths et al., 1998). This suggests that its ongoing presence is required for the maintenance of axonal support by oligodendrocytes (Lüders et 
al., 2019). The importance of PLP as a myelin component is reflected by different diseases which are associated either with mutations or overexpression such as in different forms of Pelizaeus-Merzbacher disease or are caused by the lack of PLP as in X-linked spastic paraplegia type 2 (Hobson and Kamholz, 1993; Yool et al., 2000)

Myelin oligodendrocyte glycoprotein (MOG) is a glycoprotein expressed on the oligodendrocyte cell surface and the outer tongue of the myelin sheath (Brunner et al., 1989). Due to its exposed location and high accessibility to immune cells it is frequently used as an autoantigen in immune mediated models of multiple sclerosis (Berger and Reindl, 2000; Iglesias et al., 2001; Pöllinger et al., 2009). The exact biological function of MOG is unknown but it is suggested to have adhesive function and to interact with the cytoskeleton to regulate stability of microtubules (Dyer et al., 1994; Johns and Bernard, 1999)

Myelin-associated glycoprotein (MAG) is a type I transmembrane glycoprotein that binds to gangliosides thereby maintaining the myelin-axon spacing (Pronker et al., 2016). It has adhesive functions and is primarily localized to adaxonal myelin. MAG is found in the periaxonal space along the myelin internode and implicated in axo-glial interaction, initial adhesion during myelination and signal transduction through Fyn kinase (Umemori et al., 1994; Schnaar et al., 1998; Quarles, 2007). MAG knock-out mice exhibit delayed myelination, disrupted periaxonal space and progressive axonal degeneration (Li et al., 1994; Yin et al., 1998). An interesting function of MAG in the PNS is the modulation of the axonal size. Decreased axonal caliber and reduced neurofilament spacing in PNS axons can be observed upon MAG knock-out (Yin et al., 1998).

2',3'-cyclic nucleotide 3'-phosphodiesterase (CNP) is an enzyme localized in all parts of the non-compact myelin including inner and outer tongue, cytoplasmic myelin channels and the lateral loops (Vogel et al., 1988; Snaidero et al., 2014). It is expressed early in oligodendrocyte maturation and is known to bind to the cytoskeleton and implicated in axonal survival (Lappe-Siefke et al., 2003). Recent studies have linked CNP to the maintenance and formation of cytosolic channels in myelin. CNP is promoting actin assembly by opening of cytosolic channels in myelin thereby functioning as an antagonist to myelin compaction (Snaidero et al., 2017)

\subsection{Myelin basic protein acts as molecular zipper of CNS myelin}

\subsubsection{Myelin Compaction}

By accounting for $8 \%$ of the myelin protein content MBP is the second most abundant CNS myelin protein after PLP and required for the formation of compact myelin (Vassall et al., 2015). It is an intrinsically unstructured protein with a strong positive charge of +19 at physiological pH and a high degree of random coils (Hill et al., 2002). Through its high positive charge it interacts electrostatically with negatively charged lipids in the plasma membrane such as phosphatidyl serine and PIP2 (Boggs et al., 1977). Computational modeling based on small angle X-ray scattering (Ridsdale et al., 1997) suggests that MBP folds into a $\mathrm{C}$ shape upon interaction with opposing negatively charged lipid membranes (Raasakka et al., 2017). This conformational change enables its adhesive function and allows the self-activation of MBP and in turn triggers the formation of an MBP network (Figure 1-2) (Vassall et al., 2015). This network brings myelin membranes in close 
apposition in a zipper like way and forms a molecular sieve that extrudes cytoplasm and membrane bound proteins with more then 20-30 amino acids independent of their charge from the compact myelin (Aggarwal et al., 2011b). Upon compaction, MBP is thought to be the main constituent of the major dense line (Mizuhira and Ozawa, 1967; Min et al., 2009).

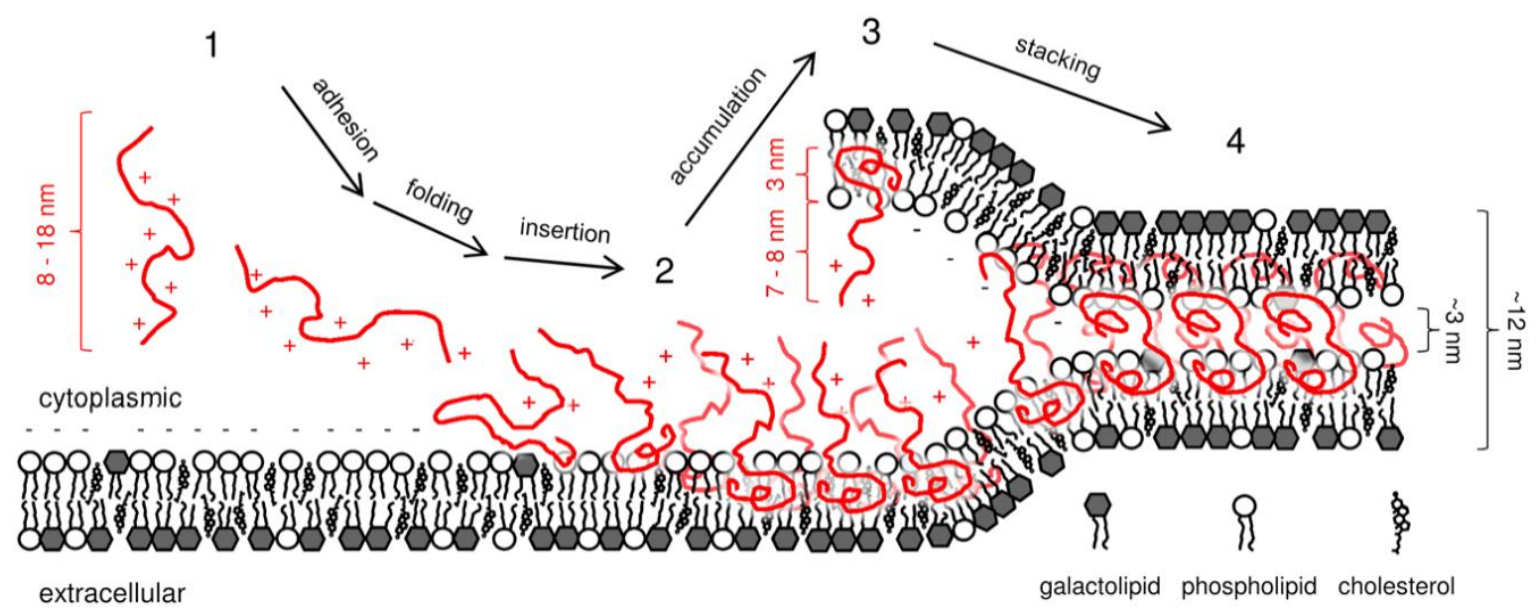

Figure 1-2 Assembly of the major dense line upon formation of compact myelin

Unfolded MBP is inserted into nascent myelin membrane and binds to the negatively charged cytoplasmic leaflet dominated by phospholipids through electrostatic interaction. Upon binding, MBP is partially integrated into the leaflet and changing its conformational state upon binding of negatively charged lipid. Once a critical threshold of MBP molecules is reached it adheres to the second cytoplasmic leaflet and folds into its adhesive C shape and forms the major dense line by extruding the cytoplasm. Adapted from Raasakka et al., 2017.

Other known functions of MBP are associated with interaction partners such as calcium dependent binding to calmodulin, a highly acidic calcium sensor (Libich and Harauz, 2008; Majava et al., 2010) and the interaction with cytoskeletal elements such as actin and tubulin (Harauz and Libich, 2009; Bamm et al., 2011). During myelination MBP is also involved in actin disassembly while CNP has been shown to promote actin assembly (Aggarwal et al., 2011b; Zuchero et al., 2015; Snaidero et al., 2017). This interplay between MBP and CNP is involved in the formation of non-compact cytosolic channels within the compact myelin which are suggested to be a transfer route for metabolites and signaling molecules (Snaidero et al., 2014, 2017). 


\subsubsection{Transcription and Translation of MBP}

The MBP gene encodes two different families of proteins: the Golli MBP isoforms (GOLLI: genes of the oligodendrocyte lineage) and the classical MBP isoforms localized in myelin. Golli MBP isoforms encode three proteins named BG21, J37 and TP8 which localize to the oligodendrocyte nucleus (Reyes and Campagnoni, 2002). These isoforms are expressed during intermediate stages of oligodendrocyte maturation and seems to be involved in the calcium homeostasis and membrane elaboration of oligodendrocytes during myelination (Campagnoni et al., 1993; Paez et al., 2007) Golli MBP isoforms are not specific to oligodendrocytes but are also expressed in neurons in the CNS and PNS (Landry et al., 1997) and in cells of the immune system such as macrophages or B cells with unknown function (Pribyl et al., 1993).

In mice six classic MBP isoforms are expressed (21.5, 20.2, 18.5, 17.24, 17.22 and $14 \mathrm{kDa})$ of which the 14 and $18.5 \mathrm{kDa}$ isoforms represent with $70 \%$ the majority of the transcripts (Barbarese et al., 1977; Akiyama et al., 2002). The 14 and $18 \mathrm{kDa}$ isoforms are known to have a high membrane affinity and are expressed late in myelination as they are required for myelin compaction. Expression of the $14 \mathrm{kDa}$ isoform alone is sufficient to rescue myelination in shiverer mice (Kimura et al., 1989). In contrast, the less abundant isoforms $17 \mathrm{kDa}$ and $21.5 \mathrm{kDa}$ localize to the nucleus of young oligodendrocytes suggesting a potential regulatory role in myelination (Allinquant et al., 1991; Pedraza, 1997). While MBP is highly conserved on a genetic level, the isoform profile in human CNS is slightly different with the following isoforms being expressed 21.5, 20.2, 18.5, 17.24, 17.22 kDa of which the $18 \mathrm{kDa}$ isoform is most abundant (Kamholz et al., 1986). MBP is also present in the PNS myelin but it is not required for peripheral myelination (Kirschner and Ganser, 1980). In the PNS the adhesive function for myelin compaction is facilitated by myelin protein P0 (Sakamoto et al., 1987; Martini et al., 1995) and potentially also by PMP22 (Suresh et al., 2010; Ruskamo et al., 2014)

In oligodendrocytes myelin associated MBP isoforms are not translated in the soma. Instead MBP mRNA is bound in RNA granules and targeted by its 3'UTR (untranslated region) to the plasma membrane and into myelinating processes and are enriched in the myelin fraction (Colman et al., 1982; Kristensson et al., 1986; Torvund-Jensen et al., 2018). Translation is triggered by phosphorylation of ribonucleoprotein A2 by Fyn kinase resulting in strictly controlled local MBP mRNA translation at sites of membrane compaction (White et al., 2008; Torvund-Jensen et al., 2014)

\subsubsection{Role of MBP in human diseases}

During its lifetime MBP can undergo a large number of post-translational modifications including phosphorylation, citrullination/deimination (modification of arginine to citrulline), deamidation (modification of an amide), acylation (addition of an acyl group) and arginine methylation (addition of an methyl group) (Zhang et al., 2012). These modifications can lower the charge of MBP resulting in decreased membrane adhesion and changes in functional properties. Modifications like acetylation and citrullination of MBP can even provoke an immune reaction (Zamvil et al., 1986; Cao et al., 1999). The strong positive charge of MBP of +19 is reduced by 1 for every added citrullinated arginine suggesting that citrullination can play a role in myelin decompaction and demyelinating diseases by lowering the binding affinity of MBP to membranes. This is supported by in vitro 
experiments where high levels of MBP citrullination have been shown to perturb membrane stacking and in vivo evidence for increased MBP citrullination after demyelination by mild cuprizone treatment (Beniac et al., 2000; Caprariello et al., 2018). The level of highly citrullinated MBP is increased 2-3 fold in chronic MS patients (Moscarello et al., 1994) and citrullinated MBP was found to be ten times more susceptible to cathepsin D degradation compared to non-citrullinated MBP (Cao et al., 1999). Up to $90 \%$ of MBP in Marburg syndrome patients and up to 45\% of MBP in chronic MS patients is highly citrullinated compared to $20 \%$ in healthy brains (Ridsdale et al., 1997) supporting a role for citrullination in demyelinating diseases.

In contrast to PLP, where several mutations are known to cause severe neurological defects like Pelizaeus-Merzbacher disease (PMD) or spastic paraplegia type 2 (SPG2) (Hobson and Kamholz, 1993), there is little evidence for human diseases associated with MBP mutation or loss. In humans MBP is localized to chromosome 18 position 18q23. Deletion of this part of chromosome 18, called 18q- syndrome or De-Grouchy-Syndrome is a rare partial monosomy that renders patients haploinsufficient for MBP. While the chromosomal deletion affects many genes the loss of MBP is considered to be responsible for delayed myelination and myelin defects in these patients as observed by magnetic resonance imaging (Ono et al., 1994; Arguedas and Batchelor, 2009). However, there is one reported case where a verified MBP haploinsufficiency does not result in an obvious loss of compact myelin despite abnormal MRI signals (Tanaka et al., 2012).

While no monogenetic disease is associated with loss of MBP its abundance is affected in some neurological disorders. Patients with schizophrenia or bipolar disorders have reduced levels of MBP in the frontal and temporal lobe and the entorhinal cortex (Chambers and Perrone-Bizzozero, 2004; Parlapani et al., 2009). The relevance of reduced MBP levels in the cortex is supported by Poggi and colleagues (Poggi et al., 2016). In their study mice heterozygous for MBP showed mostly normal behavior but were mildly hypomyelinated and had a low-grade inflammation in the prefrontal cortex. Interestingly, MBP heterozygosity led to subtle effects on sensorimotor gating in form of reduced prepulseinhibition and late-onset catatonia which hints towards a cortical network dysfunction caused by subtle myelin changes (Poggi et al., 2016). 

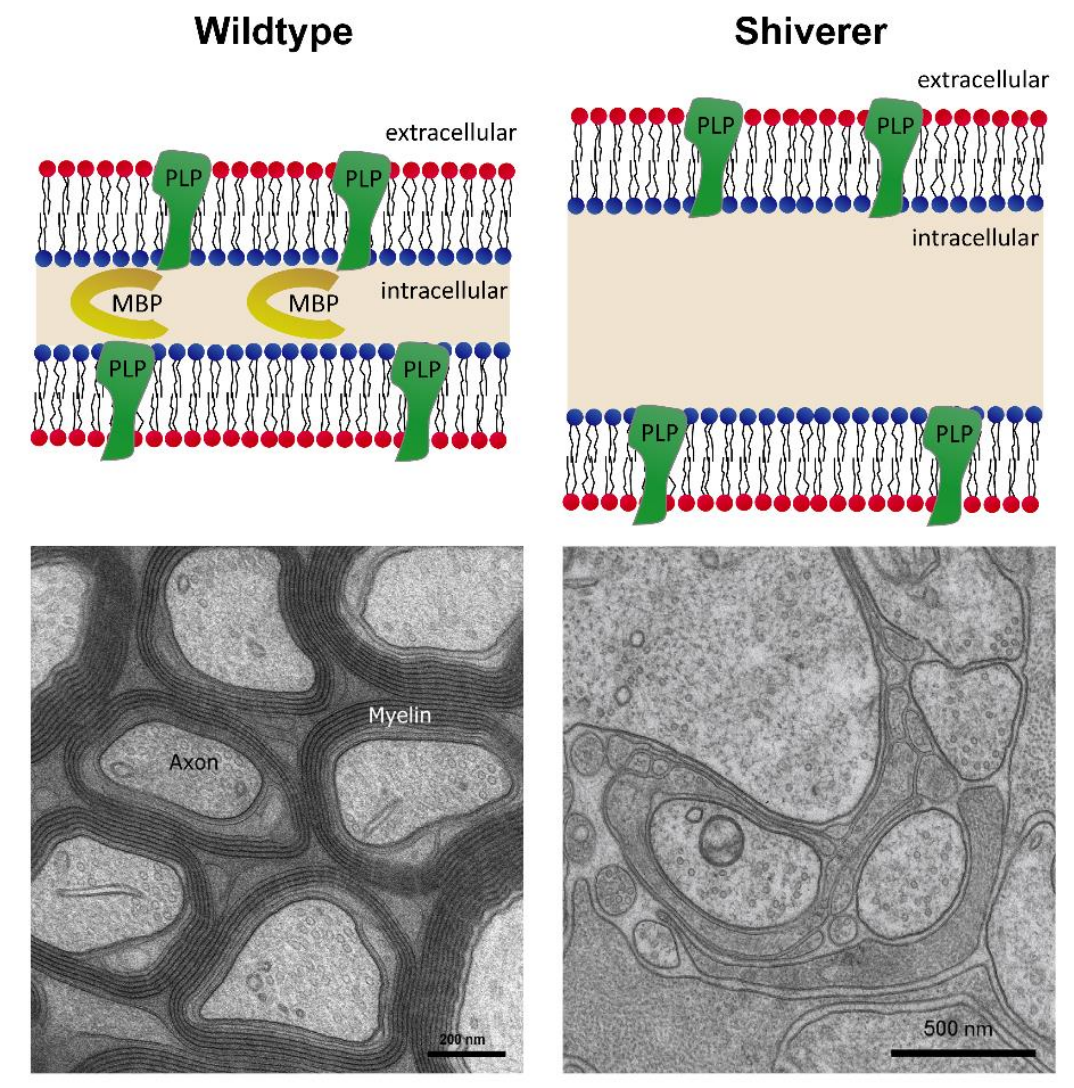

Figure 1-3 MBP is required for the formation of compact myelin

Oligodendrocytes from shiver mice are not able to form mature compact myelin membrane. Despite the lack of MBP, oligodendroglia ensheathment takes place but is not sufficient to maintain axonal insulation. TEM micrograph of High pressure frozen optic nerve at the age of 10 weeks.

\subsection{The shiverer mouse - A naturally occurring MBP mutant}

A naturally occurring mouse mutant for MBP called shiverer was described in 1977 (Doolittle and Schweikart, 1977; Chernoff, 1981). Shiverer mice have an autosomal recessive deletion of exon 2 to 7 of the classical MBP locus and are unable to form compact myelin. These mice begin to shiver at the end of the second postnatal week, the peak of myelination. During the following weeks shiverer mice develop a progressively increasing tremor, tonic convulsions and seizures before they die prematurely at an age of 8 - 11 weeks (Chernoff, 1981). Shiverer CNS has very little myelin that appears compact but lacks the major dense line. Instead non-compacted oligodendrocyte processes are present.

Despite the lack of MBP, the initial wrapping of myelin takes place with likely support by the adhesive function of other myelin proteins like PLP and MAG, resulting in non-compact myelin membranes wrapping axons (Stoffel et al., 1997) (Figure 1-3). While many MBP isoforms exist, the expression of the $14 \mathrm{kDA}$ classical MBP isoform is sufficient to rescue myelination defects in shiverer, suggesting a certain functional redundancy between isoforms (Kimura et al., 1989). A second, naturally occurring MBP mouse mutant is the socalled myelin deficient mouse ( $m l d$ ). These mice have an inversion of exon 2 in a duplicated MBP gene resulting in antisense RNA expression and reduced MBP mRNA and subsequently protein levels (Okano et al., 1987; Readhead and Hood, 1990) The shiverer phenotype could 
be recapitulated by generating mice expressing an antisense MBP construct (Katsuki et al., 1988).

The consequences of MBP knock-out are the almost complete loss of compact myelin and nodal structures as well as impaired nerve signal conduction. Decreased nerve conduction velocity in shiverer animals was measured in several white matter tracts and was reduced by $40 \%$ in spinal cord, by $20 \%$ in corpus callosum and by $60 \%$ in optic nerve (Rasband et al., 1999; Sinha et al., 2006; Windrem et al., 2008). Large caliber axons in shiverer mice show less focal sodium channel clustering (Rasband and Shrager, 2000) but increased overall sodium and potassium channel density suggesting a compensatory mechanism to preserve excitability in the absence of compact myelin (Noebels et al., 1991; Wang et al., 1995). Further changes observed in shiverer mice are decreased ganglioside and cholesterol content in CNS myelin (Bird et al., 1978; Uschkureit et al., 2000), increased proliferation of $\mathrm{NG2}^{+}$cells (Bu et al., 2004) and increased enzymatic activity of cytochrome C oxidase implicating increased mitochondrial activity in spinal cord axons (Andrews et al., 2006).

In contrast to the CNS shiverer, PNS myelin has normal periodic structure, sheath thickness and unchanged conduction velocity (Privat et al., 1979; Uschkureit et al., 2000) Only minor alterations have been observed such as slight decompaction at the inner tongue and twice as many Schmidt-Lantermann incisures (Rosenbluth, 1980; Gould et al., 1995).

Taken together the lack of MBP in shiverer mice results in severe CNS dysmyelination and axonal ion channels disorganization resulting in tremor, seizures and death within 10 weeks of age. Interestingly a double knock-out for PLP and MBP appears less affected, has more myelin layers and a longer lifetime, which led the authors to speculate that expression of PLP in the absence of MBP inhibits membrane wrapping around the axon (Stoffel et al., 1997).

\subsection{Generation of the myelin sheath}

Myelination begins at the level of oligodendrocyte progenitor cells (OPC) that are derived from neural stem and progenitor cells during late embryonic development (Kessaris et al., 2006). These cells are highly proliferative and migrate throughout the CNS. In mice formation of myelin begins at birth in the spinal cord and is mostly completed at postnatal day 60 by myelination of most brain regions (Baumann and Pham-Dinh, 2001). An essential part of this process is the synthesis of large amounts of protein and lipids. The formation of internodes happens in a matter of days and a single oligodendrocyte can produce up to 5000 $\mu \mathrm{m}^{2}$ myelin membrane with up to 160 membrane layers per day as shown in cat (Hildebrand et al., 1993, 1994; Pfeiffer et al., 1993). Through a staged maturation process (Figure 1-4) OPCs differentiate into premyelinating and then myelinating oligodendrocytes (Nave and Werner, 2014). OPCs reach out to an unmyelinated axon to form an OPC-axon contact and extend membrane processes towards the axon (Figure 1-4). The myelin sheath is divided into abaxonal myelin (outer part of the myelin sheath) and the adaxonal part of the myelin sheath, which is in close contact to the axon and comprises of the inner tongue. The nascent myelin sheath undergoes polarization mediated by PI(3,4,5)P3-dependent actin polymerization to form a growing edge followed by spiral extension of plasma membrane around the axon (Aggarwal et al., 2011a; Zuchero et al., 2015). By membrane trafficking towards the growing myelin sheath it undergoes lateral and radial expansion and new membrane is added at the inner tongue (Snaidero et al., 2014; Nawaz et al., 2015; Zuchero et al., 2015). In the abaxonal myelin MBP protein is synthesized and compaction 
progresses mostly from the abaxonal to the adaxonal site with a delay of two to three myelin layers behind the leading edge (Hildebrand et al., 1994; Snaidero et al., 2014). The leading edge of the oligodendrocytes moves underneath the already present myelin layers and extends radially and longitudinally forming an internode (Snaidero et al., 2014).

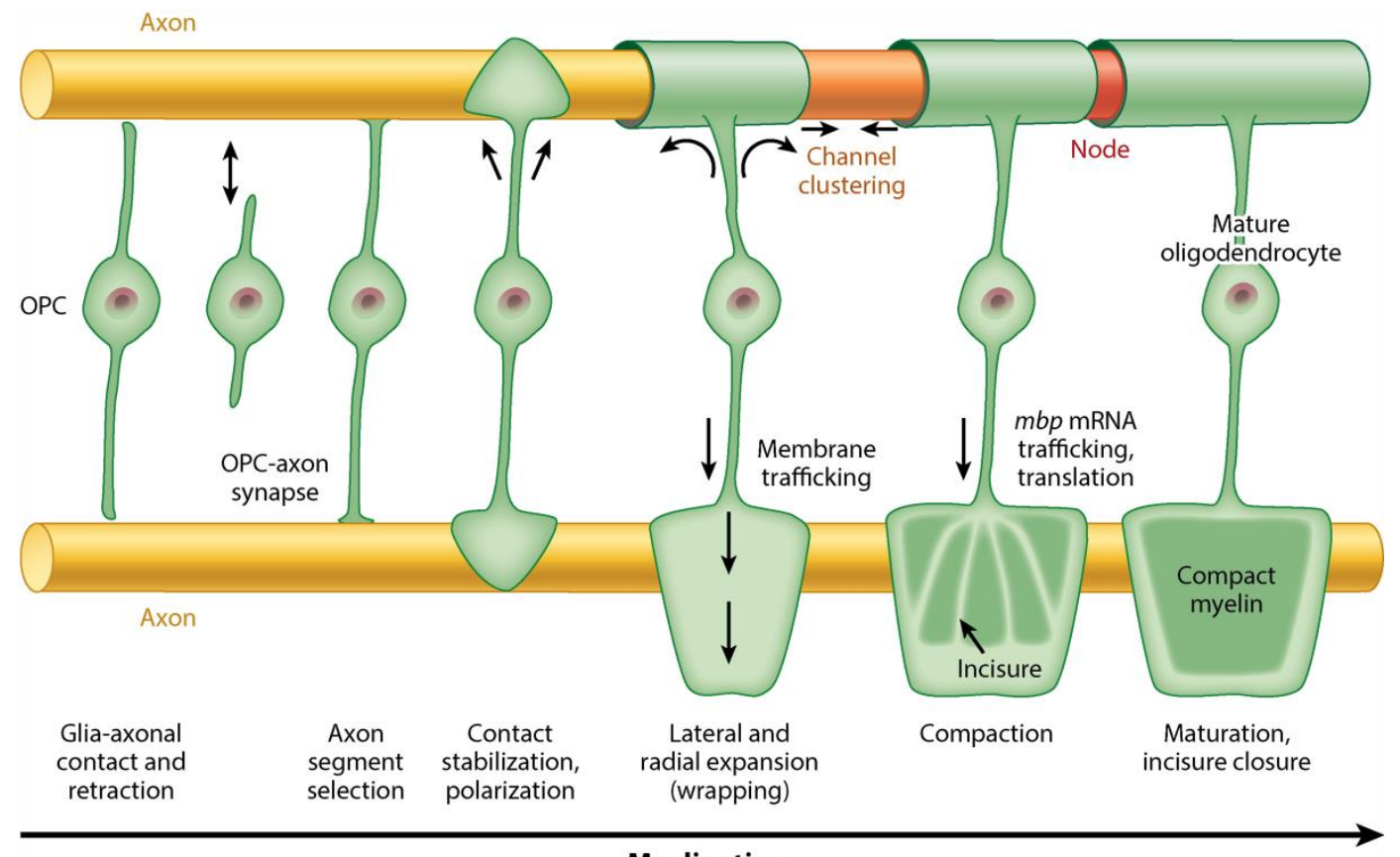

Myelination

Figure 1-4 Process of myelination in the CNS

Following migration of the OPC to the site of myelin formation, the OPC extends processes to survey the surrounding for axons that require myelination. After contact establishment the nascent myelin sheath adheres to the axon and starts lateral and radial expansion to ensheath the target axon. During ensheathment the OPC matures into a myelinating oligodendrocyte and begins to express compact myelin markers. MBP protein is then locally translated and begins to compact myelin from the abaxonal to the adaxonal sheet. Modified from Nave and Werner, 2014.

The lateral extension of the nascent myelin sheath and the covering of the axonal membrane induces the clustering of voltage gated sodium and potassium channels and formation of the node of Ranvier (Rasband and Peles, 2015). During lateral myelin sheath extension $\mathrm{Na}^{+}$ channels accumulate adjacent to the membrane processes. A node forms when $\mathrm{Na}^{+}$channel accumulations from two adjacent myelin sheets fuse to form ion channel clusters. This is facilitated by glial derived extracellular matrix that clusters NF186, the formation of axon glial junctions as diffusion barrier and the axonal emergence of cytoskeletal scaffold proteins stabilizing the $\mathrm{Na}^{+}$channels (Rasband et al., 1999; Susuki et al., 2013). 


\begin{tabular}{|c|c|c|c|}
\hline Internode & Juxta-Paranode & Paranode & $\begin{array}{c}\text { Node } \\
\text { of Ranvier }\end{array}$ \\
\hline
\end{tabular}
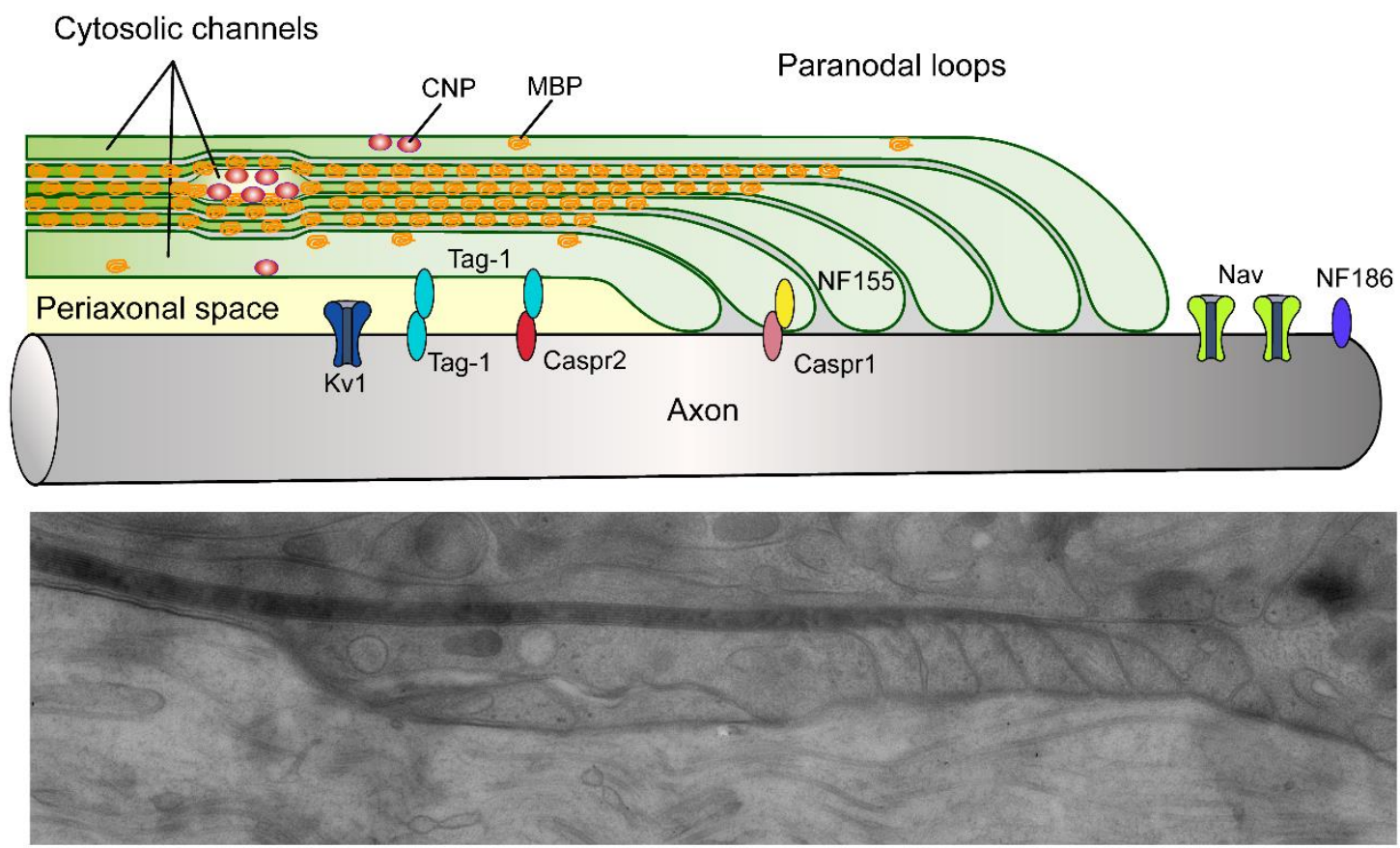

Figure 1-5 Molecular organization of Adhesion proteins at the node of Ranvier

Simplified illustration of adhesion proteins and ion channels in the axonal and oligodendrocyte membrane. (TEM micrograph: Node of Ranvier in an optic nerve longitudinal section).

The nodal region (Figure 1-5) can be divided into distinct domains: the juxta-paranode, the paranodal axoglial junctions (paranode) and the node of Ranvier with its nodal gap. The node of Ranvier is characterized by the absence of myelin and the enriched localization of sodium $\left(\mathrm{Na}^{+}\right)$and potassium $\left(\mathrm{K}^{+}\right)$channel clusters required for rapid de- and repolarization of the axonal membrane during action potential conduction. $\mathrm{Na}^{+}$channels including Nav1.1, Nav1.2 and Nav1.6 to Nav1.9 (Fjell et al., 2000; Boiko et al., 2001; Duflocq et al., 2008; Rasband and Peles, 2015) allow ion flux over the axonal membrane while the $\mathrm{K}^{+}$channels including KCNC1, KCNQ2, and KCNQ3 regulate axonal excitability (Devaux et al., 2004; Battefeld et al., 2014; King et al., 2014). Beside the described channels required for axon potential propagation the node of Ranvier is enriched in specialized cytoskeletal elements such as ankyrin $G$ and $\beta I V$-spectrin that are tethering the channels to the axonal cytoskeleton (Kordeli et al., 1995; Berghs et al., 2000). While the node is not covered by myelin some nodes are contacted by processes of perinodal astrocytes or oligodendrocyte precursors. These are suggested to buffer the perinodal extracellular ion concentration but the exact function remains to be investigated (Black and Waxman, 1988; Butt et al., 1994, 1999).

The node is directly flanked by the paranode, a specialized axonal glial contact site between axonal membrane and paranodal loops of the oligodendrocyte. Here, myelin lamellae form cytoplasmic spiraling loops that are connected to the axonal membrane by septate like junctions. The paranode is involved in the attachment of the myelin sheath to the axon and 
the electric separation of node and internode (Rosenbluth, 2009). Adhesion proteins localized at the paranode are neurofilament 155 (NF155) on the site of the oligodendrocyte and its binding partners Caspr1 as well as contactin (CNTN) on the axonal membrane (Rasband and Peles, 2015).

The juxta-paranode is covered by compact myelin and located between paranode and internode. Here, delayed rectifier potassium channels (Kv1.1 and Kv1.2) are expressed which are suggested to stabilize conduction and are involved in the maintenance of internodal resting potential during myelin formation (Chiu and Ritchie, 1980; Tao-Cheng and Rosenbluth, 1984; Arroyo et al., 2001; Chang and Rasband, 2013). Juxtaparanodal adherence is mediated by adhesion proteins TAG1 on the oligodendrocyte membrane and its respective partner CASPR2 and on the axonal membrane (Chang and Rasband, 2013). Axo-glia adhesion at the internode is achieved by the oligodendrocyte proteins MAG binding to axonal gangliosides and CADM4 binding CADM3 on the axonal side (Rasband and Peles, 2015; Elazar et al., 2019). The correct assembly and maintenance of nodes of Ranvier is essential for signal conduction. Disruption of the node of Ranvier on the glial or the neuronal site for example during autoimmune neuropathies like Guillain-Barré syndrome, toxin ingestion (Isbister and Kiernan, 2005; Yuki and Hartung, 2012) or loss of nodal components (Dupree et al., 1998) results in impaired signal conduction or even conduction block (Susuki, 2013).

\subsection{Myelin Turnover - Half-life of myelin components}

\subsubsection{Myelin protein half-life}

Once myelin has been generated and axonal wrapping is completed, myelin is considered mostly mature. This maturation is region-dependent. While most axons in the optic nerve are myelinated around postnatal day 60 (Biffiger et al., 2000), myelination is ongoing in the corpus callosum until at least postnatal day 120 (Waxman and Bennett, 1972; Remahl and Hildebrand, 1982; Yates and Juraska, 2007). Myelin that has been laid down by the oligodendrocyte and wrapped around an axon was for a long time considered stable and subject to little change.

However, aged proteins need to be removed and replaced by de novo synthesis. This replenishment is required to provide accurate protein homeostasis and to prevent the accumulation of dysfunctional proteins. The determining factor of protein turnover is the half-life or half replacement time of proteins until they are degraded. While most proteins are exchanged relatively fast within hours or days, some have been found to be extraordinary stable (Sabri et al., 1974; Price et al., 2010; Savas et al., 2012; Toyama et al., 2013; Fornasiero et al., 2018). Examples for very stable proteins with long half-lives are nucleosomes (Histone H2A up to 690 days), nuclear pore complexes (Nup188 h up to 67 days) and especially myelin proteins (e.g. MOG up to 164 days) (Fornasiero et al., 2018).

Studies from Sabri and colleagues in 1974 investigating the lifetime of individual myelin proteins found that compact myelin proteins like MBP and PLP had a high degree of "metabolic stability" (Sabri et al., 1974). However, with the given technical limitations they 
were unable to accurately calculate the half-life of MBP and considered it to be very stable (Sabri et al., 1974). Recent studies have confirmed that some of the most long lived structures reside in the myelin fraction (Toyama et al., 2013; Fornasiero et al., 2018). With extensive mathematical modeling of amino acid re-utilization, more precise half-lives of long-lived proteins have been calculated. Myelin basic protein was found to have a half-life between 82 days in cortex, 101 days in cerebellum and 70 days in a long pulse chase experiment (described in Supplementary Table 1) (Fornasiero et al., 2018) but also other myelin proteins like PLP (80 days), Claudin11 (139 days) or MOG (164 days) show a very long half-life. Other myelin proteins that reside mostly in the non-compact myelin have shorter half-lives as for example CNP with about 53 and MAG with about 36 days. In summary, compared to the previously mentioned general protein half-life of 3 to 13 days the protein components of myelin are exceptionally stable. However, compact myelin is a tightly compacted membrane structure and proteins are probably entrapped in this compartment. The protein turnover rates in myelin might also be subject to changes. Disease or aging could influence the cellular maintenance machinery and change the turnover rate of certain components (Visscher et al., 2016).

\subsubsection{Myelin Lipid half-life}

Lipids within the myelin sheath show a high lateral mobility (Dawson and Gould, 1976) and the membrane fluidity is suggested to be dependent on its cholesterol content (Rosetti et al., 2008; Maxfield and van Meer, 2010). With over 25\% total lipid content cholesterol is enriched two fold in myelin compared to other plasma membranes (van Meer et al., 2008) and its synthesis is rate limiting for myelination (Saher et al., 2005, 2009; Mathews et al., 2014). Cholesterol has a very long half replacement time of 359 days in the myelin sheath of adult mice presumably being its most stable lipid (Ando et al., 2003; Saher et al., 2005). Half replacement times for other myelin lipids including cerebroside ( 94 days), ganglioside GM1 (102 days), phosphatidylethanolamine (25 days) and phosphatidylcholine (25 days) suggest that the lipids like the protein components of the myelin sheath are relatively stable but need to undergo turnover within months after myelination (Ando et al., 2003). The mechanism of removal and replacement of these lipids is unknown; the previously mentioned high lateral mobility of lipids within the sheet might allow continuous replacement through the soma of the oligodendrocyte.

An interesting observation in the half replacement time of lipids as well as proteins is the occurrence of two pools with different turnover kinetics. Ando and colleagues concluded that in myelin of adult mice $60 \%$ of the lipids undergo rapid and $40 \%$ undergo slow exchange. For MBP it was also described that it has two pools with faster (21 days) and slower turnover (Sabri et al., 1974). It could be speculated that lipids and proteins have a certain half-replacement time directly after synthesis that is increasing as soon as they are integrated into larger stable structures like the myelin sheath.

\subsubsection{Oligodendrocyte half-life}

In rodents new oligodendrocytes are continuously generated during the adult life of an animal (Dimou et al., 2008; Rivers et al., 2008; Psachoulia et al., 2009; Kang et al., 2010; Tripathi et al., 2017). Despite the large number of cells generated, only very few of these 
cells integrate into white matter tracts and form myelin sheaths (Young et al., 2013; Tripathi et al., 2017). Adult born oligodendrocytes that do integrate differ from developmentally born oligodendrocytes as they form more but shorter myelin sheets (Young et al., 2013) and especially after remyelination also thinner myelin (Harrison et al., 1972; Gledhill and McDonald, 1977; Duncan et al., 2017). Therefore, myelination in the adult CNS probably underlies different parameters compared to developmental myelination.

Recent studies on the lifetime of oligodendrocytes in rodents show that oligodendrocytes survive for most of the life span of a mouse (Tripathi et al., 2017). The formation of new myelin and adult de novo myelination seems to be involved in learning of a motor task as shown by OPC proliferation and oligodendrocyte maturation (Young et al., 2013; McKenzie et al., 2014). Similar results have been obtained for the lifetime of oligodendrocytes in the human CNS. Based on two studies by Yeung and colleagues the majority of oligodendrocytes in the human CNS are formed until the age of 5 and survive up to 40 years (Yeung et al., $2014,2019)$. In contrast to rodents there seems to be no major proliferation of OPCs or oligodendrocytes during adulthood. Yeung and colleagues also investigated the age of oligodendrocytes in different multiple sclerosis lesions and found no signs of adult oligodendrogenesis in so called shadow plaques which are believed to be remyelinated lesions (Yeung et al., 2019). This finding supports the provocative idea that in the human CNS different to the rodent CNS, mature myelinated oligodendrocytes could be involved in and needed for remyelination (Jäkel et al., 2019; Yeung et al., 2019).

The long life of oligodendrocytes and the lack of evidence for their replacement together with the described long but finite half-lives of myelin proteins and lipids raise the question of myelin maintenance in the adult animal. Protein and lipid turnover of adult myelin are required to maintain functionality of the myelin sheath. The previously mentioned studies found that, beside the long lifetime of the oligodendrocytes, the myelin compartment itself is turned over constantly (Toyama et al., 2013; Yeung et al., 2014; Fornasiero et al., 2018). Whether this necessary turnover is achieved by removal and replacement of whole myelin sheaths or by replenishing myelin components within in a sheath remains to be determined. 


\subsection{Myelin Degradation - Removal and recycling of myelin}

To maintain the structural integrity as well as plasticity of myelin internodes the CNS needs mechanisms to turnover aged and dysfunctional components without replacing the complete oligodendrocyte. This is especially true for the myelin compartment since the lipid rich membrane is relatively inert and challenging to recycle. It is not yet clear how dysfunctional proteins during steady state turnover gets removed from compacted myelin to enter the degradative system. Here, it is necessary to distinguish two distinct situations where myelin removal is required: The loss of myelin as a pathological process in a demyelinating disease state like in multiple sclerosis, neuromyelitis optica or other demyelinating diseases (Neumann et al., 2009; Clarner et al., 2012; Romanelli et al., 2016; Weil et al., 2016) and the removal of myelin components for intrinsic turnover and normal maintenance in an functional oligodendrocyte. The removal of myelin debris after disease or injury is mediated by several cell types. During a demyelinating process, myelin is taken up by microglia (Neumann et al., 2009; Romanelli et al., 2016), astrocytes (Morizawa et al., 2017) and endothelial cells (Zhou et al., 2019).

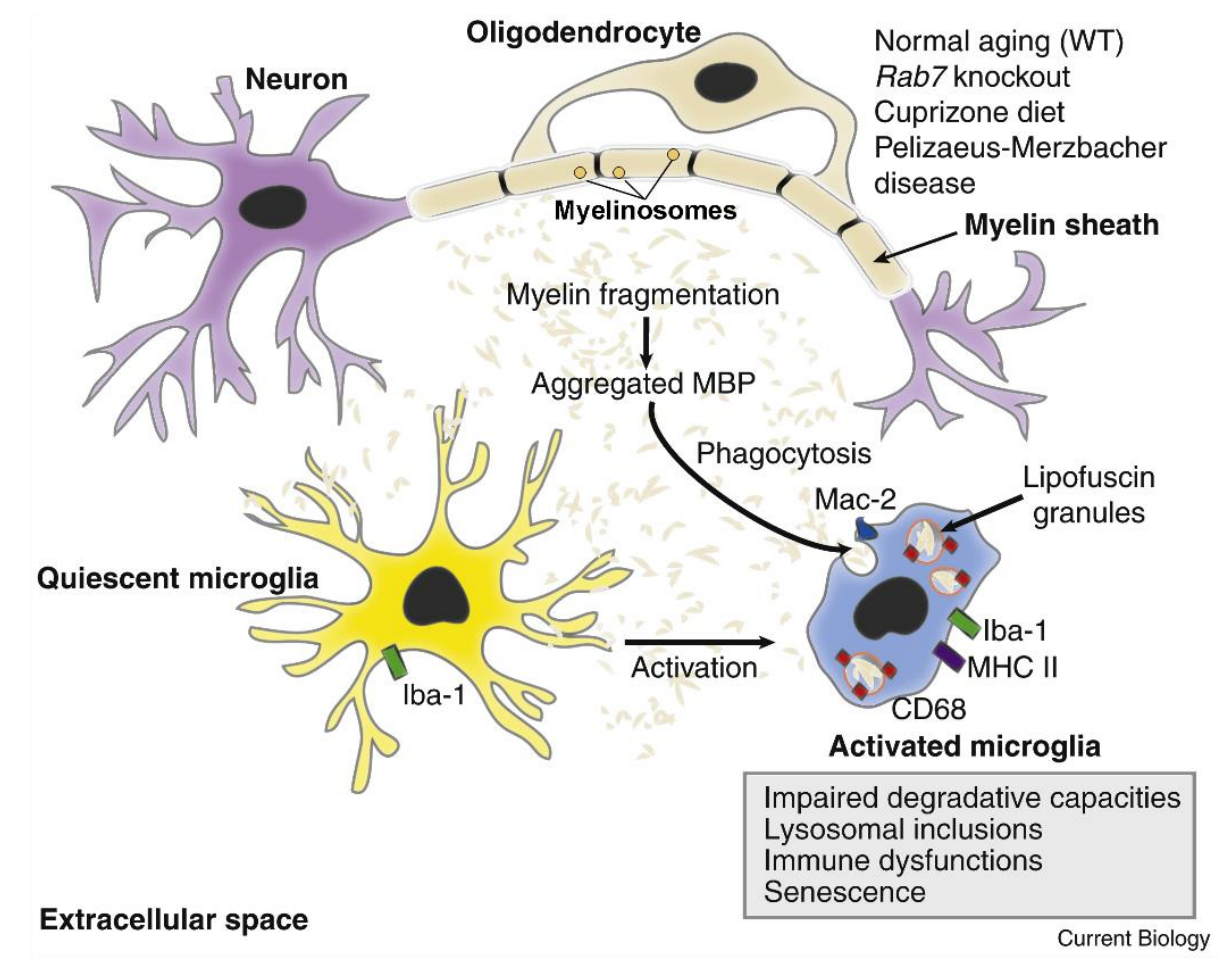

Figure 1-6 Microglial recycling of myelin fragments

Microglial activation and expression of CD68 by sensing of myelin debris followed by Mac- 2 mediated phagocytosis of myelin debris and formation of intracellular cholesterol crystals modified from Thériault and Rivest, 2016.

The removal of myelin debris (Figure 1-6) is triggered by an inflammatory microenvironment and promotes the recruitment of microglia (Kotter et al., 2006; Napoli and Neumann, 2010). Uptake of myelin debris is required to reduce break down products and clear the environment from factors that are inhibitory for remyelination (Kotter et al., 2006). Ineffective myelin debris clearance is partially responsible for the poor myelin 
regenerative ability in the CNS compared to the PNS making myelin removal an essential step in remyelination (Arandjelovic and Ravichandran, 2015). Microglial uptake of myelin debris is partially mediated via the TREM2 and the LRP1 receptor. Knock-out of TREM2 or LRP1 results in slowed myelin debris uptake and hinders remyelination (Gaultier et al., 2009; Cantoni et al., 2015; Jay et al., 2015; Poliani et al., 2015). The capacity of microglia to take up and digest myelin is not unlimited. Upon phagocytosis of large amounts of myelin, as occurring in demyelinating lesion models, microglia can form intracellular cholesterol crystals to store excessive lipids and can be visualized by electron microscopy (CantutiCastelvetri et al., 2018). The catabolic conversion of myelin fragments to lipids and metabolites might support remyelination but it is so far unclear whether oligodendrocytes have direct access to the metabolites generated by myelin degradation (Cantuti-Castelvetri et al., 2018).

The importance of microglia and also astrocytes for the clearance of myelin debris is well known (Kotter et al., 2006; Romanelli et al., 2016). However, it remains unclear whether and how microglia affect steady state myelin turnover in healthy myelin sheaths. Microglia actively take up myelin fragments released from aged myelin sheaths but this microglial myelin turnover is getting less effective with aging (Safaiyan et al., 2016). Uptake of myelin fragments, also called Marchi bodies (Hildebrand and Aldskogius, 1976; Persson and Berthold, 1991; Persson et al., 1992) or myelin spheres (Hill et al., 2018) were discussed as part of the turnover process (Persson and Berthold, 1991). How these myelin fragments are released or removed from the myelin sheath is yet unknown. However, oligodendrocytes, beside microglia and astrocytes also express LRP1 or degradation enzymes like Cathepsin D (Gaultier et al., 2009; Zhang et al., 2014) which might enable them to actively recycle myelin from their own myelin sheath.

\subsection{Models for Demyelination - myelin turnover}

Demyelinating diseases can be induced by external stimuli like toxins, viruses, traumata and by autoimmune reactions such as observed in diseases like multiple sclerosis or neuromyelitis optica. Furthermore, inherited defects such as Plp mutations in some forms of Pelizaeus-Merzbacher disease lead to demyelination (Hobson and Kamholz, 1993; Tan and Koralnik, 2010; Papadopoulos and Verkman, 2012; Graziano and Cardile, 2015). To understand these diseases different mouse models have been developed to study individual aspects of demyelinating conditions and different types of insults on the myelinating system. Experimental autoimmune encephalomyelitis (EAE) is used in mice to model autoimmune and inflammatory aspects of demyelination (Kornek et al., 2000). Paradigms to model toxin based demyelination are for example targeted injection of lysolecithin that disrupts myelin membranes (Plemel et al., 2018) or feeding of the chopper chelator cuprizone that leads to inhibition of mitochondrial complex IV, with selective toxicity for oligodendrocytes (Matsushima and Morell, 2001; Torkildsen et al., 2008). The advantage of these models is the fast demyelination ranging from weeks in EAE to days in cuprizone or lysolecithin models (Plemel et al., 2018) and the fast spontaneous remyelination within days to weeks as for example seen in the cuprizone model (Matsushima and Morell, 2001). Genetic models for demyelination include for example the targeted ablation of the myelin transcription factor myelin regulatory factor (MYRF) or the expression of the diphtheria toxin A subunit in adult oligodendrocytes (Traka et al., 2010; Koenning et al., 2012). 
These fast and rather strong effects on the myelinating system makes them excellent models to study myelin breakdown as well as remyelination, but a central hallmark of these model systems is their strong effect on oligodendroglial health and survival. The loss of oligodendrocytes in these models makes them less suitable for the investigation of steady state myelin turnover and maintenance hence new models are required to study myelin turnover.

\subsection{Project Aim}

The turnover and maintenance of myelin are important elements of the oligodendrocyte homeostasis. Throughout the long lifetime of oligodendrocytes (Yeung et al., 2014; Tripathi et al., 2017) mechanisms are required to continuously replace myelin and its components. Turnover of myelin was so far investigated on protein level by determining half-lives of proteins found in the myelin fraction. These studies have shown that myelin proteins are especially long lived and are renewed in the range of weeks to months (Fornasiero et al., 2018). However, little is known about the turnover of the adult myelin sheath itself as a structure. To investigate steady state myelin turnover a genetic model system is required that allows the visualization of turnover mechanisms under conditions of unperturbed myelin synthesis that avoids oligodendrocyte death and acute inflammation. Such a model will allow the characterization of homeostatic myelin renewal and replacement in a functional oligodendrocyte. For this study a genetic mouse model was developed that fulfils these criteria. In this thesis, I aim to investigate the site of myelin turnover within the myelin sheath. This will be approached by using the ablation of MBP synthesis in mature myelinating oligodendrocytes. The emergence of non-compact myelin membrane in the absence of MBP will be used to investigate sites of myelin component insertion into the mature myelin sheath. The detailed aims were:

1) The characterization of the newly established inducible $M b p$ knock-out model regarding motor impairment, inflammation, oligodendrocyte survival and proliferation as well as myelin gene expression to validate it as a model to study myelin turnover. 2) The characterization of any structural changes as a consequence of the ablation of MBP de novo synthesis. 3) The localization of MBP-free non-compact myelin membranes within the myelin sheath using 3D focused ion beam scanning electron microscopy (FIB-SEM) of the optic nerve to investigate the sites of membrane integration in a mature myelin sheath. 4) Characterization of the consequences of compact myelin loss in the optic nerve by proteome and lipid analysis as well as loss of axonal myelin coverage to estimate the halflife of a myelin sheath after ablation of MBP synthesis. 5) The investigation of myelin removal processes within the myelin sheath using 3D FIB-SEM.

To summarize, in this thesis I investigate the consequences of MBP synthesis ablation in mature oligodendrocytes as a tool to address aspects of myelin turnover and the maintenance of the compact myelin compartment. 


\section{Chapter 2 Material and Methods}

\subsection{Material}

Chemicals were purchased from Sigma-Aldrich GMBP, Merck KGaA or SERVA unless stated otherwise. Laboratory plastic materials were purchased from BD-Falcon (Falcon tubes), Bio-rad (Western Blot equipment), Gilson and Eppendorf (Pipettes and Eppendorf cups) or Brand (Pipet tips)

\subsubsection{Generation of the mouse model}

To facilitate the ablation of MBP the flox mouse line $\left(M b p^{f / f f l}\right.$, internal line nomenclature MBPFLX) was generated. Embryonic stem cells (ES) harboring a modified allele of the $M b p$ gene (Mbp ${ }^{\mathrm{tm} 1 \mathrm{a}}$ ) were acquired from the European Conditional Mouse Mutagenesis Program (Eucomm). ES cells were microinjected into blastocysts derived from FVB mice and embryos were transferred to pseudopregnant foster mothers. For the ES clone B02 (MBPFLX) germline transmission was achieved by breeding with C57BL/6N female mice. The resulting offspring harbored the Mbp-lacZ neo allel. The construct including the lacZ gene and a neomycin resistance cassette was excised by breeding to mice expressing a FLIP recombinase (129S4/SvJaeSor-Gt(ROSA)26Sortm1(FLP1)Dym/J; backcrossed into C57BL/6N). The resulting offspring harbored an Mbp-flox allel in which exon 1 of the classical Mbp gene is flanked by loxP sites (Figure 3-1). Genotyping of the Mbp flox allel was performed by genomic polymerase chain reaction (PCR) using the following primers (5'GGGTGATAGACTGGAAGGGTTG), (5'-GCTAACCTGGATTGAGCTTGC) and (5'- CAACGGGTTCTTCTGTTAGTCC). To inactivate Mbp expression in mature oligodendrocytes, exon 1 was excised in vivo by breeding to mice expressing an tamoxifen inducible CreErt2 under the control of the Plp promotor (Leone et al., 2003). Genotyping of the CreErt2 allele was performed using the following primers (5'-CAGGGTGTTATAAGCAATCCC), (5'-CCTGGA AAATGCTTCTGTCCG) including a primer pair for CNP as positive control (5'-GCCTTCAAACTGTCCATCTC) and (5'-CCCAGCCCTTTTATTACCAC)

\subsubsection{Statistical analysis}

Statistical analysis was performed using Graphpad Prism 7. If not stated otherwise significance was calculated by two-tailed unpaired t-test. Levels of significance were displayed as $\mathrm{p}<0.05\left(^{*}\right), \mathrm{p}<0.01\left(^{* *}\right)$ and $\mathrm{p}<0.001\left(^{* * *}\right)$. Linear regressions and one-phase decay were calculated in Graphpad Prism 7 using the mean of animals per time point.

\subsubsection{Animal handling}

All experiments were performed in accordance with the German animal welfare law and the regulations of the State of Lower Saxony for animal experiments. The animals were kept with 2-5 animals per cage in $12 \mathrm{~h}$ light dark-cycles and bred in the animal facility of the Max Planck Institute for Experimental Medicine. 


\subsubsection{Administration of Tamoxifen}

For knock-out induction 8-9W old mice were intraperitoneal injected with 1 mg Tamoxifen $(100 \mu \mathrm{l}$ of $10 \mathrm{mg} / \mathrm{ml}$ tamoxifen in corn oil) for 5 consecutive days, followed by a two-day break and 5 more days of injection. The resulting dosage is $10 \mathrm{mg}$ per mouse over a 12 day period. The day after the last tamoxifen injection was considered 0 days post tamoxifen. Mice were analyzed 2 to 12 months after tamoxifen induction.

\subsubsection{PCR and qPCR primer}

Table 1: Primers used in this thesis

\begin{tabular}{ll} 
Primer & \multicolumn{2}{l}{ Fwd Primer [5'-3'] } \\
\hline Housekeeper \\
RPS13 & CGAAAGCACCTTGAGAGGAA \\
Ppia & CACAAACGGTTCCCAGTTTT \\
RPLP0 & GATGCCCAGGGAAGACAG \\
& \\
Myelin Genes \\
Mag & AGGATGATGGGGAATACTGGT \\
Mog & ACCTGCTTCTTCAGAGACCACT \\
Cnp & CGCTGGGGCAGAAGAATAC \\
Mbp & GCCTCCGTAGCCAAATCC \\
Plp1 & CTCCAAAAACTACCAGGACTATGAG \\
Car2 & CAAGCACAACGGACCAGA \\
Olig2 & AGACCGAGCCAACACCAG \\
Pdgfr A & AAGACCTGGGCAAGAGGAAC \\
Aif1 & CCGAGGAGACGTTCAGCTAC \\
S100b & AACAACGAGCTCTCTCACTTCC \\
Aqp4 & TGGAGGATTGGGAGTCACC \\
Gfap & TGCTCCTGCTTCGAGTCCTT \\
Golli & CCTCAGAGGACAGTGATGTGTTT
\end{tabular}

Rev Primer [5'-3']

TTCCAATTAGGTGGGAGCAC

TTCCCAAAGACCACATGCTT

ACAATGAAGCATTTTGGATAATCA

AAGGATTATGGGGGCAAACT GGGGTTGACCCAATAGAAGG AAGGCCTTGCCATACGATCT GCCTGTCCCTCAGCAGATT

AGGGCCCCATAAAGGAAGA

ATGAGCAGAGGCTGTAGG

AAGCTCTCGAATGATCCTTCTTT

GAACCTGTCTCGATGGCACT

TGTTTTTCTCCTCATACATCAGAATC

CTCCATCACTTTGTCCACCA

TGAACACCAACTGGAAAGTGA

TCAAGAGGAACATCGTGGTAAAGA

AGCCGAGGTCCCATTGTT

Cholesterol genes

$\begin{array}{lll}\text { Hmgcs1 } & \text { AGCCAAAATCATTCAGGGTAAA } & \text { GGACCGCTGCTATTCTGTCT } \\ \text { Hmgcr } & \text { TGATTGGAGTTGGCACCAT } & \text { TGGCCAACACTGACATGC } \\ \text { Fdft1 } & \text { GGACCGCTGCTATTCTGTCT } & \text { TCAATCAGACCAGTCGCAGC } \\ \text { Dhc24 } & \text { GGTCATGACGGACGACGTA } & \text { AGGGCTTGTAGTAACTGCCAAT } \\ \text { Cyp51 } & \text { TTAGAACAGAAAGCAGTGTGTGG } & \text { TGCATCTATCAAGTAAATTCAGATCC } \\ \text { Srebp2 } & \text { ACCTAGACCTCGCCAAAGGT } & \text { GCACGGATAAGCAGGTTTGT } \\ \text { Fdps } & \text { TGTAAGCCGCAAACATCTTG } & \text { CCAATTTCTGGTTCCCATTC } \\ \text { Apoe } & \text { GACCCTGGAGGCTAAGGACT } & \text { AGAGCCTTCATCTTCGCAAT } \\ \text { Apod } & \text { ACGGAAACATCGAAGTGCTAA } & \text { TGGCTTCACCCTTTACTTGG } \\ \text { Ldlr } & \text { GATGGCTATACCTACCCCTCAA } & \text { TGCTCATGCCACATCGTC }\end{array}$




\subsubsection{Antibodies}

Table 2: Primary antibodies used in this thesis

\begin{tabular}{|c|c|c|c|}
\hline Antigen & Host species & Method & Manufacturer \\
\hline Actin & Monoclonal mouse & WB (1:5000) & Millipore \\
\hline APP & Monoclonal mouse & Paraffin IHC (1:750) & Chemicon \\
\hline CD3 & Monoclonal rat & Paraffin IHC (1:150) & Abcam \\
\hline CNPase & Monoclonal mouse & Paraffin IHC (1:150) & $\begin{array}{l}\text { Sigma } \\
\text { Clone 11-5B }\end{array}$ \\
\hline Iba1 & Polyclonal goat & Paraffin IHC (1:200) & $\begin{array}{l}\text { Abcam } \\
\text { ab5076 }\end{array}$ \\
\hline Mac3 & Monoclonal rat & Paraffin IHC (1:500) & BD Pharmingen 553322 \\
\hline MBP & Monoclonal rat & Cryo IHC (1:400) & Serotec MCA409S \\
\hline MBP & Polyclonal rabbit & $\begin{array}{l}\text { IHC }(1: 200) \\
\text { WB }(1: 500)\end{array}$ & $\begin{array}{l}\text { Dako A0623 } \\
\text { Discontinued }\end{array}$ \\
\hline MBP & Polyclonal rabbit & $\begin{array}{l}\text { IHC }(1: 4000) \\
\text { IEM: }(1: 250)\end{array}$ & $\begin{array}{l}\text { Custom made (Kathrin } \\
\text { Kusch) }\end{array}$ \\
\hline Nav 1.6 & Polyclonal rabbit & Cryo IHC (1:500) & Alomone, ASC-009 \\
\hline Caspr1 & Monoclonal mouse & Cryo IHC (1:500) & Neuromabs \\
\hline GFAP & Monoclonal mouse & Paraffin IHC (1:200) & Novocastra \\
\hline $\begin{array}{l}\text { PLP } \\
\text { (A431) }\end{array}$ & Polyclonal rabbit & Paraffin IHC (1:500) & $\begin{array}{l}\text { K.-A. Nave } \\
\text { custom made A431 }\end{array}$ \\
\hline Olig2 & Monoclonal mouse & Paraffin IHC (1:100) & $\begin{array}{l}\text { Gift from J. Alberta, } \\
\text { custom made }\end{array}$ \\
\hline CAII & Polyclonal rabbit & Paraffin IHC (1:150) & $\begin{array}{l}\text { Gift from Sahid G. } \\
\text { custom made }\end{array}$ \\
\hline PDGFR $\alpha$ & Monoclonal Rabbit & Paraffin IHC (1:500) & Cell Signaling 3174 \\
\hline
\end{tabular}


Table 3: Secondary antibodies used in this thesis

\begin{tabular}{llll}
\hline $\begin{array}{l}\text { Antibody } \\
\alpha \text {-rabbit-HRP }\end{array}$ & $\begin{array}{l}\text { Host species } \\
\text { Goat }\end{array}$ & $\begin{array}{l}\text { Method } \\
\text { IHC }(1: 10.000)\end{array}$ & $\begin{array}{l}\text { Manufacturer } \\
\text { Dianova }\end{array}$ \\
$\alpha$-mouse-HRP & Goat & IHC $(1: 10.000)$ & Dianova \\
$\begin{array}{l}\alpha \text {-mouse-Alexa } \\
\text { 488, 555, 594 or } 633\end{array}$ & Donkey & IHC $(1: 1000)$ & Dianova \\
$\begin{array}{l}\alpha \text {-rabbit-Alexa } \\
\text { 488, 555,594 or } 633\end{array}$ & Donkey & IHC $(1: 1000)$ & Dianova \\
$\alpha$-rat-Alexa 488,555 & Donkey & IHC $(1: 1000)$ & Dianova
\end{tabular}

\subsubsection{General buffers}

All buffers are prepared using $\mathrm{ddH}_{2} \mathrm{O}$ (deionized milli q-water) if not stated otherwise.

\section{Phosphate buffered saline (PBS)}

10X PBS stock solution (1L):

$1370 \mathrm{mM} \mathrm{NaCL} \quad[80.0 \mathrm{~g}]$

$27 \mathrm{mM} \mathrm{KCl} \quad[2.0 \mathrm{~g}]$

$80 \mathrm{mM} \mathrm{Na}_{2} \mathrm{HPO}_{4} \quad[14.4 \mathrm{~g}]$

$20 \mathrm{mM} \mathrm{KH}_{2} \mathrm{PO}_{4} \quad$ [2.4 g]

pH was adjusted to 7.1 using $\mathrm{NaOH}$

\section{0x Tris-buffered saline (TBS) (1L)}

$500 \mathrm{mM}$ Tris/HCl, pH $7.5 \quad[60.6 \mathrm{~g}]$

$1.5 \mathrm{M} \mathrm{NaCl} \quad$ [87.6 g]

\section{Protein biochemistry buffer}

\section{Modified RIPA Buffer}

$1 \mathrm{x}$ TBS

$1 \mathrm{mM}$ EDTA

$0.5 \%[\mathrm{w} / \mathrm{v}]$ Sodium deoxycholate

$1.0 \%[\mathrm{v} / \mathrm{v}]$ Triton X-100

cOmplete $^{\mathrm{Tm}}$ Mini protease inhibitor (Roche Diagnostics $\mathrm{GmbH}$ )

1 tablet per $10 \mathrm{ml}$ of RIPA buffer

Protease inhibitor was added freshly to the RIPA buffer before use. 
4X SDS-Sample buffer $(10 \mathrm{ml})$

$1.67 \mathrm{ml} 1.5 \mathrm{M}$ TRIS $\mathrm{pH} 6.8$

$80 \mu \mathrm{l} 0.5$ M EDTA pH 8.0

$0.8 \mathrm{~g}$ SDS

$2 \mathrm{ml} 0.2 \%$ bromphenol blue

$400 \mu \mathrm{l} \beta$-mercaptoethanol

$4 \mathrm{ml}$ glycerol

Added up to $10 \mathrm{~mL}$ with ddH2O and stored at $-20^{\circ} \mathrm{C}$

1x TBS with Tween-20 (TBS-T for WB)

$50 \mathrm{mM}$ Tris/HCl, pH 7.5

$150 \mathrm{mM} \mathrm{NaCl}$

$0.05 \%[\mathrm{v} / \mathrm{v}]$ Tween-20

10x SDS running buffer (Laemmli buffer) (1L)

$250 \mathrm{mM}$ Tris base $\quad[144 \mathrm{~g}]$

$1.92 \mathrm{M}$ Glycine $\quad[30.28 \mathrm{~g}]$

$1 \%[\mathrm{w} / \mathrm{v}] \mathrm{SDS} \quad[10 \mathrm{~g}]$

Transfer buffer (1L)

$39 \mathrm{mM}$ Tris base $\quad$ [29.25 g]

$48 \mathrm{mM}$ Glycine $\quad[58.148 \mathrm{~g}]$

$10 \%[\mathrm{v} / \mathrm{v}]$ Methanol $[100 \mathrm{ml}]$

\section{Milk blocking buffer for WB}

$5 \%[\mathrm{w} / \mathrm{v}]$ non-fat dry milk powder (Hema) in $1 \mathrm{x}$ TBS-T

\section{Protein marker for SDS-Page}

PageRuler Plus Prestained Protein Ladder 10-250kDa (Thermo Scientific, Cheshire, UK)

\section{Buffers and reagents for electron microscopy}

\section{Phosphate buffer (PB):}

$0.1 \mathrm{M}$ phosphate buffer (PB) was prepared as follows (1L):

$0.04 \mathrm{M} \mathrm{NaH}_{2} \mathrm{PO}_{4} \cdot \mathrm{H}_{2} \mathrm{O}$

[1.8 g]

$0.16 \mathrm{M} \mathrm{Na}_{2} \mathrm{HPO}_{4} \cdot 2 \mathrm{H}_{2} \mathrm{O}$

[15.5 g]

Volume was added up to $1 \mathrm{~L}$ with $\mathrm{ddH}_{2} \mathrm{O}$ 


\title{
Karlsson and Schultz buffer 0.2M Stock solution:
}

0.2 M Karlsson and Schultz phosphate buffer was prepared as follows (1L):

$3.6 \mathrm{~g} \mathrm{NaH} \mathrm{PO}_{4} \cdot \mathrm{H}_{2} \mathrm{O}$

$31 \mathrm{~g} \mathrm{Na}_{2} \mathrm{HPO}_{4} \cdot 2 \mathrm{H}_{2} \mathrm{O}$

$10 \mathrm{~g} \mathrm{NaCl}$

Volume was added up to $1 \mathrm{~L}$ with $\mathrm{ddH}_{2} \mathrm{O}$

\section{Karlsson-Schultz fixative (Electron microscopy)}

$4 \%[\mathrm{w} / \mathrm{v}] \mathrm{PFA}$

$2.5 \%[\mathrm{v} / \mathrm{v}]$ Glutaraldehyde

In 0.1 M Karlsson and Schultz phosphate buffer

\author{
Immuno Karlsson-Schultz fixative (Cryo-Immuno Electron microscopy) \\ $4 \%[\mathrm{w} / \mathrm{v}]$ PFA \\ $0.25 \%[\mathrm{v} / \mathrm{v}]$ Glutaraldehyde \\ In 0.1 M Karlsson and Schultz phosphate buffer
}

\section{Durcupan resin for FIB-SEM sample embedding}

Durcupan is a resin mixture used for samples embedded for FIB-SEM microscopy. In comparison to Epon it forms a harder and more inert resin. It is less prone to waving artifacts during cross-section milling and interacts less with the electron and the ion beam which results in less imaging artifacts in FIB-SEM milling and imaging. The Durcupan kit (Durcupan $^{\mathrm{TM}}$ ACM, 44610, Sigma-Aldrich) consists of 4 components that are mixed on a magnetic stirrer.

For $43.4 \mathrm{~g}$ Durcupan the following components were combined:

Component A, M epoxy resin (Sigma-Aldrich, 44611)

Component B, hardener 964 (Sigma-Aldrich, 44612)

$20 \mathrm{~g}$

Component C, accelerator 960 (DY 060) (Sigma-Aldrich, 44613)

$0.6 \mathrm{~g}$

Then $0.2 \mathrm{~g}$ component $\mathrm{D}$ was added as accelerator and crosslinker. The resulting mixture was slowly stirred for additional 20 mins before use.

\section{Epoxy Resin (Epon) for TEM sample embedding:}

For $47.1 \mathrm{~g}$ Epon the following components were mixed on a magnetic stirrer

Glycidether 100 (Serva, 21045)

$21.4 \mathrm{~g}$

2-Dodecenylsuccinic acid anhydride (DDSA) (Serva, 20755) $\quad 14.4 \mathrm{~g}$

Methylnadic anhydride(MNA) (Serva, 29452)

$11.3 \mathrm{~g}$

Solution was slowly stirred for 15 mins.

Then $0.84 \mathrm{~mL}$ 2,4,6-Tris(dimethyl-aminomethyl-)-phenol (DMP-30) (Serva) was added as accelerator. The resulting mixture was slowly stirred for additional $20 \mathrm{~min}$ before use. 


\section{Formvar solution}

$612.5 \mathrm{mg}$ Formvar (Plano, Wetzlar, Germany) is dissolved in $50 \mathrm{~mL}$ Chloroform and stirred for at least 30 minutes. The resulting solution is stored at RT protected from light.

\section{Richardson's Methylene blue / Azure II blue}

Staining solution for lipid-rich regions in electron microscopy semi thin sections. Stock solutions were prepared as follows:

$1 \%$ [w/v] Azure II (Merck, Darmstadt, Germany) in ddH2O (stock solution I)

$1 \%[\mathrm{w} / \mathrm{v}]$ Methylene Blue (Merck, Darmstadt, Germany) in $1 \%$ sodium borate (stock solution II)

For a working solution equal volumes of the stock solutions were mixed in a syringe and filtered directly before use. Working solution was used for a maximum of $24 \mathrm{~h}$.

\section{Immunohistochemistry buffers:}

\section{Paraformaldehyde}

$4 \%[\mathrm{w} / \mathrm{v}]$ Paraformaldehyde

In $0.1 \mathrm{M}$ Phosphate buffer

\section{Citrate buffer (0.01 M, pH 6.0) (Immunohistochemistry)}

$1.8 \mathrm{mM}$ Citric acid $\left(\mathrm{C}_{6} \mathrm{H}_{8} \mathrm{O}_{7} \cdot \mathrm{H}_{2} \mathrm{O}\right)$

$8.2 \mathrm{mM}$ Sodium citrate $\left(\mathrm{C}_{6} \mathrm{H}_{5} \mathrm{O}_{7} \mathrm{Na}_{3} \cdot 2 \mathrm{H}_{2} \mathrm{O}\right)$

Citrate buffer was always prepared fresh

\section{Triple-Blocking solution for immunohistochemistry}

For $100 \mathrm{ml}$ Stock solution (100\%) the following blocking reagents were diluted in $100 \mathrm{~mL} 1 \mathrm{X}$ PBS:

$2 \mathrm{~mL}$ Fetal calf serum (FCS)

$2 \mathrm{~g}$ Bovine serum albumin (BSA)

$2 \mathrm{~g}$ fish skin gelatin

\section{$10 \%$ BSA solution for immunocytochemistry}

For a 10\% stock solution $10 \mathrm{~g}$ Bovine serum albumin (BSA) were diluted in $100 \mathrm{ml} 1 \mathrm{x}$ PBS.

\section{Tris buffer (pH 7.6)}

$50 \mathrm{mM}$ Tris/HCl, $\mathrm{pH} 7.6$

$0.9 \%[\mathrm{w} / \mathrm{v}] \mathrm{NaCl}$ 


\section{Mounting media}

Eukitt Kindler for DAB labeled sections (Kindler, Freiburg, Germany)

Aqua-Poly/Mount Polysciences for fluorescence labeled sections (Polysciences, Eppelheim, Germany)

\subsubsection{Manufacturer Kits}

Kits were used according to the manufacturer's guidelines

Table 4: Commercial kits used in this thesis

\begin{tabular}{|c|c|c|}
\hline Kit & Usage & Manufacturer \\
\hline Pierce BCA protein assay & $\begin{array}{l}\text { Protein quantification for } \\
\text { WB }\end{array}$ & $\begin{array}{l}\text { Thermo Scientific, } \\
\text { Cheshire, UK }\end{array}$ \\
\hline RNAeasy mini prep & RNA isolation & Quiagen, Hilden, Germany \\
\hline $\begin{array}{l}\text { SuperScript III First-Str. } \\
\text { Synth. }\end{array}$ & $\begin{array}{l}\text { Synthesis of cDNA from } \\
\text { RNA }\end{array}$ & Invitrogen \\
\hline DAB Zytomed Kit & DAB IHC & Zytomed Systems GMBH \\
\hline $\begin{array}{l}\text { Click-iT EdU Alexa Fluor } \\
647\end{array}$ & $\begin{array}{l}\text { EdU detection in paraffin } \\
\text { sections }\end{array}$ & $\begin{array}{l}\text { (Thermo Scientific, } \\
\text { Cheshire, UK) }\end{array}$ \\
\hline
\end{tabular}

Adult Brain Dissociation Kit Oligodendrocyte isolation mouse and rat with Anti-04 from adult mouse brain MicroBeads, and MS

Columns
Miltenyi Biotec $\mathrm{GmbH}$, Bergisch Gladbach, Germany 


\subsubsection{Software}

Table 5: Software used in this thesis

\begin{tabular}{lll} 
Software & Application & Manufacturer/Developer \\
\hline $\begin{array}{l}\text { Adobe Photoshop } \\
\text { CS5 }\end{array}$ & Image processing & Adobe \\
GraphPad Prism 7 & $\begin{array}{l}\text { Statistical analysis and data } \\
\text { visualization }\end{array}$ & www.graphpad.com \\
ImageJ & $\begin{array}{l}\text { Image processing and } \\
\text { quantification }\end{array}$ & $\begin{array}{l}\text { NIH, } \\
\text { http://rsbweb.nih.gov/ij/ }\end{array}$ \\
ZEN & Image processing and export & Zeiss \\
LasAF & Image processing and export & Leica \\
Imaris & 3D Data visualization & Bitplane \\
Microscopy Image & EM data segmentation \& analysis & www.mib.helsinki.fi/ \\
Browser (MIB) & & \\
IMOD 4.9 & EM data segmentation & University of Colorado \\
& & Boulder \\
Excel 2013 & Data analysis & Microsoft \\
Matlab 2016 & Data analysis & Mathworks \\
Illustrator CS5 & Figure design & Adobe \\
Rstudio 1.2 & Data analysis & RStudio Inc. \\
& &
\end{tabular}




\subsection{Methods}

\subsubsection{Protein analysis}

\subsubsection{Tissue lysis}

For whole brain Lysate, one hemisphere was homogenized in $4 \mathrm{ml}$ modified RIPA buffer using a T-10 basic Ultra-Turrax (IKA, IKA®-Werke GmbH \& CO. KG, Staufen Germany) for 20-30 sec on speed setting 3-4. The homogenate was then centrifuged at $13.000 \mathrm{rpm}$ for 10 min at $4^{\circ} \mathrm{C}$ and the supernatant was transferred to a new tube. Protein concentration was determined in triplicates using the BCA protein assay kit (Pierce) according to the manufacturer's manual.

\subsubsection{Sodium Dodecyl Sulfate Polyacrylamide Gel Electrophoresis}

Proteins were separated according to their electrophoretic mobility and size by Sodium Dodecyl Sulfate Polyacrylamide Gel Electrophoresis (SDS-PAGE). $1.5 \mathrm{~mm}$ thick Gels were self-casted using the BioRad system and contained a stacking and a $10 \%$ or $12 \%$ separation gel (Table 6). Samples for loading were prepared with equal protein amounts, mixed with SDS-sample buffer as well as $5 \%$ [v/v] mercaptoethanol and heated to $40^{\circ} \mathrm{C}$ for 10 minutes before loading. As a size reference $3 \mu \mathrm{l}$ of the PageRuler Plus pre-stained protein ladder (Thermo Scientific, Cheshire, UK) were loaded in the outermost left lane. Electrophoresis was performed in a Biorad gel chamber filled with running buffer at $200 \mathrm{~V}$ until the dye front left the gel. Gels were subsequently used for Western blotting to detect proteins of interest by using antibodies.

\subsubsection{Western blot}

To transfer proteins from the SDS-PAGE gel onto a PVDF Membrane (Amersham) a BioRad WetBlot chamber was used. The PVDF membrane was activated in $100 \%$ [v/v] methanol for $1 \mathrm{~min}$ then washed two times in $\mathrm{ddH}_{2} 0$ before transfer. The gel and the respective membrane together with 4 filter papers on each side were transferred into a BioRad WetBlot chamber and transfer was carried out for $45 \mathrm{~min}$ at $38 \mathrm{~V}$ in $1 \mathrm{x}$ transfer buffer. To prevent nonspecific antibody binding, membranes were blocked in 5\% non-fat dry milk in TBS-T for $1 \mathrm{~h}$ at RT. After blocking a primary antibody against the protein of interest was diluted in $5 \%$ non-fat dry milk in $1 \mathrm{x}$ TBS with $0.1 \%$ Tween 20 (TBS-T) and incubated overnight at $4^{\circ} \mathrm{C}$ on a rotator. On the next day the membrane was washed three times for at least 15 min each with TBS-T followed by incubation with a horseradish peroxidaseconjugated secondary antibody (dilution 1:10.000) in fresh 5\% non-fat dry milk in TBS-T for $1 \mathrm{~h}$ at RT on a rotator. After incubation the membrane was washed three times for 15 mins with TBS-T to remove unbound antibody. The membrane was developed using an advanced fluorescent and ECL Imager (Intas) and the Enhanced Chemiluminescence (ECL) Immunoblot detection kit Western Lightning (Perkin Elmer Life Sciences). The detected signals were saved as TIF files and analyzed using Image (NIH) and compared to an appropriate loading control to determine relative protein amounts. 
Table 6 Polyacrylamide gel preparation

\begin{tabular}{|c|c|c|}
\hline Reagent & $\begin{array}{l}\text { Stacking Gel } \\
{[1 \text { gel }]}\end{array}$ & $\begin{array}{l}\text { Separation Gel }(12 \%) \\
\text { [1 gel] }\end{array}$ \\
\hline $\mathrm{ddH}_{2} \mathrm{O}$ & {$[1.025 \mathrm{ml}]$} & {$[3.9 \mathrm{ml}]$} \\
\hline Acrylamid/ & $4 \%[\mathrm{w} / \mathrm{v}]$ & $12 \%[\mathrm{w} / \mathrm{v}]$ \\
\hline Bisacrylamid 29:1 & {$[0.325 \mathrm{ml}]$} & {$[3.725 \mathrm{ml}]$} \\
\hline 0.5M Tris HCL pH 6.8 & $\begin{array}{l}125 \mathrm{mM} \text { Tris} / \mathrm{HCl} \mathrm{pH} 6.8 \\
{[0.625 \mathrm{ml}]}\end{array}$ & - \\
\hline 1.5M Tris HCL pH 8.8 & - & $\begin{array}{l}0.4 \mathrm{M} \text { Tris } / \mathrm{HCl} \mathrm{pH} 8.8 \\
{[3.25 \mathrm{ml}]}\end{array}$ \\
\hline $\begin{array}{l}10 \% \text { Sodium Dodecyl } \\
\text { Sulfate }\end{array}$ & - & $\begin{array}{l}0.1 \%[\mathrm{w} / \mathrm{v}] \mathrm{SDS} \\
{[92 \mu \mathrm{l}]}\end{array}$ \\
\hline $10 \%$ Ammonium-persulfate & $\begin{array}{l}0.05 \%[\mathrm{w} / \mathrm{v}] \text { APS } \\
{[12.5 \mu \mathrm{l}]}\end{array}$ & $\begin{array}{l}0.03 \%[\mathrm{w} / \mathrm{v}] \text { APS } \\
{[31.25 \mu \mathrm{l}]}\end{array}$ \\
\hline $\begin{array}{l}\text { Tetramethylethylene- } \\
\text { diamine (TEMED) }\end{array}$ & $\begin{array}{l}0.1 \%[\mathrm{v} / \mathrm{v}] \text { TEMED } \\
{[2.5 \mu \mathrm{l}]}\end{array}$ & $\begin{array}{l}0.08 \%[\mathrm{v} / \mathrm{v}] \text { TEMED } \\
{[7.5 \mu \mathrm{l}]}\end{array}$ \\
\hline
\end{tabular}

\subsubsection{Proteom analysis of optic nerves}

Whole optic nerves were homogenized in $250 \mu$ ice cold modified RIPA buffer using teflon beads and a Precellys 24 tissue homogenizer (Bertin instruments, France). Homogenisation was carried out at a frequency of 5500 for $2 \times 10$ seconds. Samples were subsequently centrifuged at $13.000 \mathrm{rpm}$ at $4{ }^{\circ} \mathrm{C}$ and the supernatant was collected to reduce undissolved tissue and cellular nuclei. The protein concentration of the supernatant was determined in triplicates using the BCA protein assay kit (Pierce).

For quality control $0.5 \mu \mathrm{g}$ protein of each sample were loaded on a gel and SDS-PAGE with subsequent silver staining of the gel to detect protein band (Blum et al., 1987; Mortz et al., 2001). Samples were then submitted to the proteome facility of the Max-Planck-Institute of Experimental Medicine headed by Dr. Olaf Jahn and processed as described previously (Ambrozkiewicz et al., 2018). Two digestion replicated of four optic nerves lysates per group and condition were measured by liquid chromatography coupled to electrospray mass spectrometry (LC-MS). Post identification analysis and peptide identification was performed by Dr. Olaf Jahn using ISOQuant (http://www.isoquant.net). Statistical evaluation of significant changes in protein abundance was performed by Lars Piepkorn using moderated t-statistics based on calculation of false discovery rates for multiple testing correction using the bioconductor packages "limma" and "q-value" in the Rstudio environment (Storey and Tibshirani, 2003; Ritchie et al., 2015). For heatmap visualization both digestion replicate measurements were averaged to receive 4 biological replicates per condition and time point then. $\log _{2}$ fold changes between control and knock-out were 
calculated and visualized in heatmaps where red represents increased and blue decreased protein abundance.

Go-Term enrichment analysis was performed using the functional profiling feature (g:GO St) of g-profiler (https://biit.cs.ut.ee/gprofiler/). Proteins were sorted by abundance and separated into increased $(>0)$ and decreased $(<0)$ abundance relative to control ( $\log _{2}$ Foldchange). Only proteins with a significantly changed abundance were used for enrichment (cut off threshold q-value $<0.05$ ). Protein Database accession IDs were used as identifiers and compared to the mus musculus background. The gSCS threshold was used to describe the significance of enrichment together with an additional used defined 0.05 threshold. The top 20 enriched pathways of upregulated gene obtained from the KEGG database were visualized including the adjusted enrichment p-values (Kanehisa and Goto, 2000; Kanehisa et al., 2017, 2019).

\subsubsection{Lipidome analysis}

For lipid analysis, mice $8 \mathrm{~W}$ pti and $16 \mathrm{~W}$ pti were sacrifice by cervical dislocation followed by extraction of optic nerve and brain. Complete optic nerves were directly transferred in $0.5 \mathrm{ml}$ Precellys tubes filled with Teflon beads and placed on dry ice. Brains were sectioned in $300 \mu \mathrm{m}$ slices in ice cold PBS using a vibratome (VT1200S, Leica, Vienna, Austria) and four $1.5 \mathrm{~mm}$ diameter biopsy punches (Harries Unicore $1.5 \mathrm{~mm}$, GE Healthcare, United Kingdom) of caudal corpus callosum from 3 consecutive sections were transferred to $0.5 \mathrm{ml}$ Precellys tubes and immediately placed on dry ice. Mass spectrometry measurements were performed by Prof. Britta Brügger (Biochemistry center at the university of Heidelberg (BZH) as described previously (Özbalci et al., 2013) using a QTRAP 6500 LC-MS/MS System. A lipidome profile was obtained for optic nerve and corpus callosum of 4 animals per condition at $8 \mathrm{~W}$ and $16 \mathrm{~W}$ pti.

\subsubsection{Isolation of oligodendrocytes by magnetic cell isolation (MACS)}

Isolation of oligodendrocytes from mice $16 \mathrm{~W}$ pti was performed in collaboration with Lena Spieth, Stefan Berghoff and Tim Düking (Group of Gesine Saher, Max-Planck-Institut of Experimental Medicine). Oligodendrocytes were isolated by Lena Spieth according to the manufacturers protocol (Adult Brain Dissociation protocol, Miltenyi Biotec GmbH, Bergisch Gladbach, Germany). Corpus callosum was dissected using a brain matrix, manually reduced to small pieces using a scalpel and transferred to round bottom tubes with appropriate amount of dissociation enzymes mix 1 on ice. After dissection enzyme mix 2 was added and samples were dissociated at $37^{\circ} \mathrm{C}$ for $30 \mathrm{~min}$. Cells were then washed with PBS and passed through a $70^{\circ} \mu \mathrm{m}$ strainer and centrifuged (Heraeus Biofuge Pico table centrifuge) at $300 \mathrm{~g}$ for $10 \mathrm{~min}$ at $4^{\circ} \mathrm{C}$. The pellet was resuspended in PBS + Debris Removal Solution and centrifuged at $3000 \mathrm{~g}$ for $10 \mathrm{~min}$ at $4^{\circ} \mathrm{C}$. The upper two phases were removed washed with PBS and centrifuged at $1200 \mathrm{~g}$ for $10 \mathrm{~min}$. The pellet was then incubated in $1 \mathrm{x}$ Red blood cell removal solution and after $10 \mathrm{~min}$ washed with PBS and centrifuged at $3000 \mathrm{~g}$ for $10 \mathrm{~min}$ at $4^{\circ} \mathrm{C}$. The cell pellet was resuspended in appropriate amounts according to the antibody Microbead kit protocol. To label the oligodendrocyte fraction the pellet was incubated with $2.5 \mu \mathrm{l}$ anti-04 Microbeads in $97.5 \mu \mathrm{l}$ PBS for $15 \mathrm{~min}$. For removal of unbound 
Microbeads $1 \mathrm{ml}$ PBS was added followed by centrifuged at $300 \mathrm{~g}$ for $10 \mathrm{~min}$ at $4^{\circ} \mathrm{C}$. Cells were then resuspened in $0.5 \mathrm{ml}$ PBS. MS Columns used for magnetic isolation were attached to the Octomacs magnet, equilibrated with $0.5 \mathrm{ml}$ PBs and loaded with microbead cell suspension. The columns were washed three times with PBS and detached from the magnet. Purified oligodendrocytes were then eluted in $0.5 \mathrm{ml}$ of RLT buffer for mRNA isolation and stored at $-80^{\circ} \mathrm{C}$. Due to low RNA yield, cDNA amplification by single primer isothermal amplification (SPIA) from NuGEN was performed according to the manufacturers protocol.

\subsection{5 mRNA analysis}

\subsubsection{RNA-Isolation and cDNA synthesis}

Tissue was homogenized in ice cold $4 \mathrm{ml}$ QIAzol Lysis Reagent (Qiagen, Hilden, Germany) using a T-10 basic Ultra-Turrax (IKA, IKA®-Werke GmbH \& CO. KG, Staufen Germany) for 20-30 sec on speed setting 3-4 according the manufacturers guidelines. The Ultra-Turrax was cleaned with $70 \%$ EtOH and $\mathrm{H}_{2} \mathrm{O}$ between every homogenization. Corpus callosum samples were homogenized in $1 \mathrm{ml}$ QIAzol using teflon beads and a Precellys 24 tissue homogenizer. Homogenisation was carried out at a frequency of 5500 for $2 \times 10$ seconds. Samples homogenized in QIAzol are stable at RT. Of each sample $1 \mathrm{ml}$ was collected, mixed with $200 \mu \mathrm{l}$ chloroform, briefly vortexed and incubated for 2-3 min at RT. Samples were then centrifuged at $13.000 \mathrm{rpm}$ for $15 \mathrm{~min}$ (Heraeus Biofuge Pico table centrifuge). The transparent upper aqueous phase of approximately $700 \mu \mathrm{l}$ was transferred to a new tube and an equal volume of fresh $70 \% \mathrm{EtOH}$ was added. The volume was well mixed by pipetting and transferred to a RNeasy Mini column (RNeasy Mini Kit, Quiagen, Hilden, Germany). RNA was then isolated according to the manufactures protocol and resuspended in 30-100 $\mu \mathrm{l}$ Rnase free water. Quality and amount the isolated RNA were determined by using the Agilent RNA 6000 Nano KIT and the Agilent 2100 Bioanalyzer following the company's guidelines. Only RNA with an integrity index of at least 8.5 was used for further experiments.

\subsubsection{2 cDNA synthesis}

To quantify the amount of RNA transcripts via qRT-PCR the isolated RNA was converted into single stranded cDNA. First-strand cDNA synthesis was carried out via a reverse transcription reaction catalyzed by the RNA dependent DNA polymerase SuperScript III (Invitrogen). In a first step, primer and random nonamers are added to the RNA. Mix 1: $800 \mathrm{ng}$ RNA in $8 \mu \mathrm{l}$ Rnase free water, $2 \mu \mathrm{ldT}$ mix Primer $(0.6 \mathrm{pmol} / \mu \mathrm{l}$ ) and $2 \mu \mathrm{l} 9$ (random nonamers $120 \mathrm{pmol} / \mu \mathrm{l}$ ). This first mix was then incubated for $10 \mathrm{~min}$ at $70^{\circ} \mathrm{C}$ to denature RNA and primers followed by a 1 min incubation on ice.

Then, $4 \mu \mathrm{l} 5 \mathrm{x}$ first strand buffer, $1 \mu \mathrm{l}$ dNTP $(10 \mathrm{mM}), 2 \mu \mathrm{LDT}(100 \mathrm{mM})$ and $1 \mu \mathrm{l}$ SuperScript III reverse transcriptase $(200 \mathrm{U} / \mu \mathrm{l})$ were added. The complete mixture was incubated in a thermocycler with the following protocol: $25^{\circ} \mathrm{C} 10 \mathrm{~min}, 50^{\circ} \mathrm{C} 45 \mathrm{~min}, 55^{\circ} \mathrm{C} 45 \mathrm{~min}$. The synthesized cDNA was then diluted to $5 \mathrm{ng}$ per $\mu \mathrm{l}$ and stored at $-20^{\circ} \mathrm{C}$.

\subsubsection{Quantitative real time PCR (qRT-PCR)}

qRT-PCR was used to determine the relative amount of the transcript of interest for each sample. Analysis was performed using SYBR Green Master Mix according to the 
manufacturer's instructions (Applied Biosystems, Darmstadt, Germany). qRT-PCR was performed with the following mixture per well: $5 \mu \mathrm{l}$ 2x SYBR Green Master Mix, 10 ng cDNA in $2 \mu \mathrm{l}$ rnase free water, $0.4 \mu \mathrm{l}$ forward $(10 \mathrm{pmol} / \mu \mathrm{l})$ and $0.4 \mu \mathrm{l}$ reverse $(10 \mathrm{pmol} / \mu \mathrm{l})$ primer and $2.8 \mu \mathrm{l}$ rnase free water.

Primers were designed using the Universal Probe Library form Roche Applied systems (https://www.roche-applied-science.com) and validated using NIH PrimerBlast (www.ncbi.nlm.nih.gov/tools/primer-blast/). Each reaction was performed in triplicates using the following qRT-PCR program: Initial $95^{\circ} \mathrm{C}$ for $10 \mathrm{sec}$ to activate the enzyme followed by 45 cycles of $95^{\circ} \mathrm{C}$ for $15 \mathrm{sec}$ and $60^{\circ} \mathrm{C}$ for $1 \mathrm{~min}$ on a LightCycler $\AA 480$ Instrument II (Roche). Relative mRNA abundance was quantified using the LightCycler ${ }^{\circledR} 480$ Instrument II software and expressed as relative fold change of control. mRNA Abundance was, if not stated otherwise, normalized to RPS13 and Ppia.

\subsubsection{Histological analysis}

\subsubsection{Tissue collection}

Animals designated for histological analysis were sedated by i.p. injection of Avertin $(0.2 \mathrm{ml}$ per $10 \mathrm{~g}$ of body weight) and after confirmed sedation prepared for transcardial perfusion. The lower abdomen was opened with surgical scissors, the diaphragm was removed and a butterfly needle (27G, Venofix) was inserted into the left ventricle of the heart while the right atrium was opened to allow blood to leave the circulatory system. A piece of tail was removed for later re-genotyping. Then, a peristaltic pump was used to perfuse the circulatory system at a speed of $1 \mathrm{~mL} / \mathrm{min}$ for $5 \mathrm{~min}$ with HBSS at RT (Invitrogen, Karlsruhe, Germany) to flush out blood followed by 10 to 15 min of perfusion with $4 \%$ PFA in $0.1 \mathrm{M} \mathrm{PB}$ to fix the tissue.

\subsubsection{Immunohistochemistry of cryostat sections}

For cryostat sectioning mice were perfused with 4\% PFA in $0.1 \mathrm{M}$ PB and the extracted organs were kept in fixative for post-fixation for $10 \mathrm{~min}$ (optic nerve) or $12 \mathrm{~h}$ (brain and spinal cord). After fixation, tissue was incubated for cryoprotection for 2-3 days in $30 \%$ sucrose at $4^{\circ} \mathrm{C}$. Tissue that was successfully infused with sucrose sank to the bottom of the used container. The tissue was then covered in Tissue-Tek ${ }^{\circledR}$ O.C.T. Compound (Sakura ${ }^{\circledR}$ Finetek) for stabilization and was frozen and stored at $-20^{\circ} \mathrm{C}$ to $-80^{\circ} \mathrm{C}$. A cryostat (Leica, Vienna, Austria) was then used to cut sections of stabilized tissue. Optic nerve sections of 8$9 \mu \mathrm{m}$ were prepared. The sections were collected on glass slides, dried at $37^{\circ} \mathrm{C}$ for $1 \mathrm{~h}$ and then stored at $4^{\circ} \mathrm{C}$ or $-20^{\circ} \mathrm{C}$. For immunofluorescent labeling, the sections were washed three times 10 min in PBS followed by permeabilization for one hour in $0.4 \%$ Triton-100 in PBS. Sections were blocked for $1 \mathrm{~h}$ in 1:2 triple-blocking solution for immunohistochemistry diluted in 1x PBS. The primary antibody was then diluted in the blocking solution and incubated overnight at $4^{\circ} \mathrm{C}$. On the next day, slides were washed thrice with $1 \mathrm{x}$ PBS for 15 min each and incubated with the fluorescently labeled secondary antibody for $1 \mathrm{~h}$ at RT. After the incubation the slides were once again washed thrice with 1x PBS for 15 min each, incubated with DAPI for $20 \mathrm{~min}$ and washed again before being mounted using AquaPoly/Mount (Polysciences). Sections where stored at $4^{\circ} \mathrm{C}$ until imaging. 


\subsubsection{Confocal and epifluorescence imaging}

Fluorescent slides were imaged on an Axio-Observer epifluorescence microscope (Zeiss) using the software ZEN. For imaging a UV-lamp and filter cubes specific for the appropriate fluorophore were used.

Fluorophore Filter Cube

Alexa 488 GFP

Alexa 555 dsRed

Alexa $633 \quad$ Cy5

Confocal images were acquired on a SP5 Leica confocal microscope. Fluorescent signals were imaged sequentially to avoid cross bleeding using an HCX PL APO lambda blue 63.0x1.20 WATER UV objective. The following laser lines were used for the specific fluorophores:

Argon Laser at $488 \mathrm{~nm}$ was used to excite Alexa 488 Argon Laser at $514 \mathrm{~nm}$ was used to excite Alexa 555

A HeliumNeon (HeNe) laser at 633nm was used to excite Alexa 633 and Alexa 647.

The confocal software LasAF was used for image acquisition and resulting images were saved as .lif and quantified using FIJI.

\subsubsection{Paraffin embedding of tissue and Immunohistochemistry}

For paraffin embedding mice were perfused with 4\% PFA in $0.1 \mathrm{M}$ PB and the extracted organs were kept in fixative for post-fixation of $12 \mathrm{~h}$. After fixation, tissue was embedded in paraffin (Paraplast, Leica, Wetzlar, Germany) using an automated embedding system (HMP 110, MICROM).

Protocol for paraffin embedding:

$\begin{array}{lll}50 \%[\mathrm{v} / \mathrm{v}] & \text { Ethanol } & 1 \times 1 \mathrm{~h} \\ 70 \%[\mathrm{v} / \mathrm{v}] & \text { Ethanol } & 2 \times 2 \mathrm{~h} \\ 96 \%[\mathrm{v} / \mathrm{v}] & \text { Ethanol } & 2 \times 2 \mathrm{~h} \\ 100 \%[\mathrm{v} / \mathrm{v}] & \text { Ethanol } & 2 \times 2 \mathrm{~h} \\ 100 \% & \text { Isopropanol } & 1 \times 1 \mathrm{~h} \\ 100 \% & \text { Xylol } & 2 \times 2 \mathrm{~h} \\ 100 \% & \text { Paraffin } & 2 \times 2 \mathrm{~h}\end{array}$

After infusion with paraffin the samples were positioned in metal forms filled with paraffin and casted into paraffin blocks. The blocks were transferred to a cooling plate to solidify and afterwards stored at RT. Blocks were then cut in $5 \mu \mathrm{m}$ thick sections using a manual microtome (HM 400, MICROM). Sections were transferred to a $37^{\circ} \mathrm{C}$ water bath to unfold and collected on glass slides. At least two sections were collected per slide as replicates for later analysis. Slides with sections were dried overnight at $37^{\circ} \mathrm{C}$ and stored at room temperature. 


\subsubsection{Immunohistochemistry of Paraffin embedded sections}

Before labeling, paraffin sections were deparaffinized by the following protocol

$\begin{array}{ll}\text { Melting of surrounding paraffin at } 60^{\circ} \mathrm{C} & 10 \mathrm{~min} \\ \text { Xylol } 100 \% & 10 \mathrm{~min} \\ \text { Xylol } 100 \% & 10 \mathrm{~min} \\ \text { Xylol } 50 \%+\text { Isopropanol } 50 \% & 10 \mathrm{~min} \\ \text { Ethanol } 100 \%[\mathrm{v} / \mathrm{v}] & 5 \mathrm{~min} \\ \text { Ethanol } 90 \%[\mathrm{v} / \mathrm{v}] & 5 \mathrm{~min} \\ \text { Ethanol } 70 \%[\mathrm{v} / \mathrm{v}] & 5 \mathrm{~min} \\ \text { Ethanol } 50 \%[\mathrm{v} / \mathrm{v}] & 5 \mathrm{~min} \\ \text { ddH2O } & 5 \mathrm{~min}\end{array}$

Deparaffinized $5 \mu \mathrm{m}$ section were then used to label the histopathological markers GFAP, Mac3, CD3 and APP using chromogenic detection, Carboanhydrase II, Olig2 and PDGFRA were labeled using antibodies conjugated to fluorophores.

For fluorescent or DAB labeling deparaffinized section were transferred into glass cuvettes and incubated in citrate buffer for $5 \mathrm{~min}$. Section were then transferred into a plastic cuvette filled with hot citrate buffer and boiled for $10 \mathrm{~min}(600 \mathrm{~W}$, microwave) to facilitates heat induced antigen retrieval as well as permeabilization. After boiling the section were left to cool in citrate buffer for $20 \mathrm{~min}$. Then, sections were rinsed with Tris buffer containing $2 \%$ non-fat dry[w/v] milk powder for $5 \mathrm{~min}$ and fixed into Shandon coverplates (Thermo Scientific, Cheshire, UK).

Sections used for DAB labeling were treated by applying $100 \mu \mathrm{l} 3 \%$ [v/v] hydrogen peroxide for $5 \mathrm{~min}$ endogenous peroxidase activity in the section was inhibited. This is necessary to prevent endogenous peroxidases from converting the later used DAB substrate into nonspecific background signal. The sections were then rinsed three times with Tris buffer containing $2 \%[\mathrm{w} / \mathrm{v}]$ non-fat dry milk powder. Slides were then incubated in the blocking solution $(20 \%$ [v/v] goat serum in PBS/BSA [1\%]) for 10 to $30 \mathrm{~min}$ at RT. The used primary antibody was diluted in PBS/BSA [1\%], applied to the slides fixed in coverplates and incubated overnight at $4^{\circ} \mathrm{C}$. On the next day, the sections were rinsed with Tris buffer containing $2 \%[\mathrm{w} / \mathrm{v}]$ non-fat dry milk powder.

Sections used for fluorescent labeling were incubated with secondary antibody conjugated to a fluorophore (1:1000) and DAPI (1:10.000 of a $1 \mathrm{mg} / \mathrm{ml}$ Dapi stock solution) in PBS/BSA [1\%] for $1 \mathrm{~h}$ at RT. After incubation section were rinsed 3x 10 min with Tris buffer containing $2 \%[\mathrm{w} / \mathrm{v}]$ non-fat dry milk powder. For embedding sections were mounted in Aqua-Poly/Mount (Polysciences) and stored at $4^{\circ} \mathrm{C}$.

Sections used for DAB labeling were instead incubation with $100 \mu$ of biotinylated bridging antibody from the LSAB2 kit (Dako, Hamburg, Germany) for 10 min at RT and rinsed with tris buffer containing $2 \%[\mathrm{w} / \mathrm{v}]$ non-fat dry milk powder. A horseradish peroxidase (HRP) coupled secondary antibody was applied and incubated for 10 min followed by a rinsing step with Tris buffer containing $2 \%$ [w/v] non-fat dry milk powder. The HRP substrate 3,3'Diaminobenzidine (DAB) from the the DAB Zytomed Kit (Zytomed Systems GmbH, Berlin, Germany) was applied and incubated for 10 min. Brown DAB precipitate developed in the section at sites where the antigen of interest was detected by the set of primary followed by secondary antibodies with HRP. Sections were washed twice with $\mathrm{ddH}_{2} \mathrm{O}$ for $5 \mathrm{~min}$ and 
incubated for $5 \mathrm{~min}$ in $0.1 \%$ [w/v] Haematoxylin to label nuclei in blue. After two rinsing steps with $\mathrm{ddH}_{2} 0$, sections were briefly incubated in HCL-Alcohol $(0.09 \%$ [v/v] HCL, 70\% [v/v] Ethanol), rinsed in $\mathrm{ddH}_{2} \mathrm{O}$ and incubated for $5 \mathrm{~min}$ in Scott's solution $(0.2 \%[\mathrm{w} / \mathrm{v}]$ Potassiumhydrogencarbonate $2 \%[\mathrm{w} / \mathrm{v}]$ Magnesium sulphate). After a rinsed in $\mathrm{ddH}_{2} 0$, slides were incubated in $0.1 \%$ [w/v] Eosin $\left(0.1 \%\right.$ [v/v] Eosin in $\left.\mathrm{ddH}_{2} 0\right)$ for 3-5 min as counterstaining and rinsed in $\mathrm{dd}_{2} \mathrm{O}$. For embedding and longtime storage tissue was dehydrated via an increasing ethanol series with $5 \mathrm{~min}$ incubation for the following steps $(50 \%[\mathrm{w} / \mathrm{v}], 70 \%[\mathrm{w} / \mathrm{v}], 90 \%[\mathrm{w} / \mathrm{v}]$, and $100 \%[\mathrm{w} / \mathrm{v}])$, incubation in Xylol/Isopropanol (1:1) followed by two times Xylol and mounted in Eukitt (EUKITT, o-kindler, Germany)

\subsubsection{Myelin purification}

This purification protocol is based on a method developed by Norton and Poduslo to isolate a compact myelin fraction by ultracentrifugation and sucrose gradients (Norton and Poduslo, 1973; Larocca and Norton, 2007) and is performed as previously described (Jahn et al., 2013; Erwig et al., 2019). Buffers, solutions and glassware were cooled to $4^{\circ} \mathrm{C}$ and all preparation steps were performed on ice. Myelin purification was performed on a maximum of 6 samples per preparation as limited by the number of buckets fitting into the ultracentrifuge.

Using a Kinematica AG Polytron PT 3000 homogenizer, half a mouse brain was completely homogenized in 0,32 M sucrose supplemented with protease inhibitors (Roche Complete, Roche Diagnostics GmbH, Mannheim, Germany). Brain homogenates were then stored on ice until all 6 samples were homogenized. $300 \mu$ of homogenate per brain were collected and stored at $-80^{\circ} \mathrm{C}$. New Ultra-Clear centrifuge tubes (Beckman No. 344060) were filled with $0,85 \mathrm{M}$ sucrose. The brain homogenate was then carefully layered on top of the $0,85 \mathrm{M}$ sucrose using a $10 \mathrm{ml}$ pipette. For all following centrifugation steps the tubes were carefully adjusted for tara down to $0.01 \mathrm{~g}$ to avoid any imbalances. The homogenate filled tubes were then centrifuged for $30 \mathrm{~min}$ at $70000 \mathrm{~g}$ in a swing out rotor (SW40Ti Beckman rotor, $24.400 \mathrm{rpm}$ ) in a Sorvall WX ultracentrifuge (Thermo Scientific, Cheshire, UK). After centrifugation the tubes were kept on ice and the white interface between the sucrose layers was collected by using a fine glass pasteur glass pipette and transferred to a new clean tube. Water was added to perform a first osmotic shock and the tubes were centrifuge for $15 \mathrm{~min}$ at $70.000 \mathrm{~g}$. The resulting pellet was resuspended in water for $15 \mathrm{~min}$ (second osmotic shock) and centrifuged at $12000 \mathrm{~g}$ for $15 \mathrm{~min}$ (SW40Ti Beckman rotor, 9700rpm). The resulting pellet was then resuspended in $0,32 \mathrm{M}$ sucrose. A new tube was filled with $0,85 \mathrm{M}$ sucrose and the resuspended pellet was layered on top as before. Tubes were then centrifuged for $30 \mathrm{~min}$ at $70000 \mathrm{~g}$ in a swing out rotor (SW40Ti Beckman rotor, $24.400 \mathrm{rpm}$ ). The resulting interphase was collected by using a fine glass pasteur glass pipette and transferred to a new clean tube. Water was added to the tube to wash the interphase followed by $15 \mathrm{~min}$ centrifugation at $70000 \mathrm{~g}$ (SW40Ti Beckman rotor, $24.400 \mathrm{rpm}$ ). The resulting pellet contained a purified myelin fraction and was resuspended in $200 \mu \mathrm{l} 1 \mathrm{x}$ TBS containing protease inhibitors and stored at $-80^{\circ} \mathrm{C}$ (Roche Complete, Roche Diagnostics GmbH, Mannheim, Germany). 


\subsubsection{EdU labeling of proliferating cells in vivo}

To assess the proliferation of oligodendrocytes after induction of MBP knock-out animals were supplied with 5-ethynyl-2'-deoxyuridine (EdU) in drinking water. This thymidine analog incorporates into the DNA of dividing cells and allows the visualization of cell that divided during the feeding period. Male mice 32 and 40 weeks after tamoxifen injection were supplied with $0.2 \mathrm{mg} / \mathrm{ml} \mathrm{EdU} \mathrm{in} \mathrm{drinking} \mathrm{water} \mathrm{for} 3$ weeks. Water was prepared freshly every 2-3 days and animals consumed between 6 and $9 \mathrm{ml}$ per mouse per day. Animals were transcardially perfused for histological analysis 3 weeks after EdU administration was finished to allow for differentiation of the labeled cells. Paraffin sections of coronal brain slices were prepared as described previously. Brain slices were deparaffinized and labeled for EdU using the Click-iT EdU Alexa Fluor 633 (Thermo Scientific, Cheshire, UK) according to the manufacturer's protocol. After EdU labeling the paraffin sections were labeled for CAII, Olig2 or PDGFRA as described previously (2.2.6.4) .

\subsubsection{Electron microscopy}

\subsubsection{Conventional Fixation}

Animals designated for ultrastructural analysis were transcardially perfused for 5 min with HBSS followed by 10-15 min perfusion with Karlsson-Schultz fixative (see 2.2.6.1). The perfused tissue was post-fixed in Karlsson-Schultz fixative for at least $12 \mathrm{~h}$.

In case only parts of the animal were collected for ultrastructural analysis, animals were sacrificed by cervical dislocation. Optic nerves were removed from the brain and directly immersed in Karlsson-Schultz fixative. Larger tissues like brain or spinal cord were collected, immersed in cold PBS and cut on a vibratome (VT1200S, Leica, Vienna, Austria). Brain was cut into $200 \mu \mathrm{m}$ thick sagittal sections, spinal cord was coronally cut into $100 \mu \mathrm{m}$ sections and immersion fixed in Karlsson-Schultz fixative for at least $12 \mathrm{~h}$ before embedding.

\subsubsection{Conventional embedding}

Karlsson-Schultz fixed samples were transferred to $0.1 \mathrm{M} \mathrm{PB}$ and the region of interest was isolated by using a biopsy punch (Harries Unicore $1.5 \mathrm{~mm}$, GE Healthcare, United Kingdom). Punches or whole optic nerves were then wrapped in tea filter paper and sorted into segmented plastic baskets (Science Services, Munich, Germany), transferred into a Lynx Tissue Processor II (Science Services, Munich, Germany) and prepared for embedding using the following protocol: 
Table 7 Conventional embedding for EM

\begin{tabular}{|c|c|c|}
\hline Reagent & Time & Temperature \\
\hline $0.1 \mathrm{M} \mathrm{PB}$ & $\begin{array}{l}3 \times 10 \\
\min \end{array}$ & $4^{\circ} \mathrm{C}$ \\
\hline $2 \% 0 s 04$ & $4 \mathrm{~h}$ & $4^{\circ} \mathrm{C}$ \\
\hline $0.1 \mathrm{M}$ PB & $\begin{array}{l}3 \times 10 \\
\min \end{array}$ & $4^{\circ} \mathrm{C}$ \\
\hline $30 \%$ acetone & $20 \mathrm{~min}$ & $\mathrm{RT}$ \\
\hline $50 \%$ acetone & $20 \mathrm{~min}$ & RT \\
\hline $70 \%$ acetone & $20 \mathrm{~min}$ & RT \\
\hline $90 \%$ acetone & $20 \mathrm{~min}$ & RT \\
\hline $100 \%$ acetone & $10 \mathrm{~min}$ & $\mathrm{RT}$ \\
\hline $100 \%$ acetone & $10 \mathrm{~min}$ & RT \\
\hline $100 \%$ acetone & $10 \mathrm{~min}$ & RT \\
\hline $100 \%$ acetone & $10 \mathrm{~min}$ & RT \\
\hline acetone /Epon 2:1 & $2 \mathrm{~h}$ & RT \\
\hline acetone /Epon 1:1 & $2 \mathrm{~h}$ & $\mathrm{RT}$ \\
\hline acetone /Epon 1:2 & $4 \mathrm{~h}$ & RT \\
\hline Epon $100 \%$ & $4 \mathrm{~h}$ & RT \\
\hline
\end{tabular}

After dehydration and Epon infiltration optic nerves were transferred into embedding molds and covered with Epon resin. Punches from spinal cord and brain were placed flat on parafilm-covered glass slides and covered with Epon-filled gelatin capsules. All blocks were polymerized for $24 \mathrm{~h}$ at $60^{\circ} \mathrm{C}$.

\subsubsection{Modified ROTO embedding}

Samples used for FIB-SEM imaging fixed with Karlsson-Schultz fixative were embedded using a high heavy metal protocol to enhance contrast and signal of membrane in the SEM. The reduced Osmium/TCH/Osmium protocol (ROTO) is based on Mikula and colleagues (Mikula et al., 2012) and was modified for improved myelin preservation and visualization in the FIB-SEM. Optic nerves were wrapped in tea filter paper and transferred into segmented plastic baskets (Science Services, Munich, Germany) Baskets were mounted on a metal rod for handling during embedding and the following protocol was performed by hand:

\section{Reagents:}

Reduced 0sO ${ }_{4}: \quad 50 \%$ PB $(0,2 \mathrm{M})+45 \%$ OsO4 $(4 \%)+5 \% \mathrm{~K} 3[\mathrm{Fe}(\mathrm{CN}) 6](10 \%)$

TCH: $\quad 0.1 \mathrm{~g}$ thiocarbohydrazide in $10 \mathrm{ml} \mathrm{ddH_{2 } \mathrm { O }}$

Os0 $4: \quad 50 \%$ PB $(0,2 \mathrm{M})+50 \%$ OsO4 (4\%) 
Table 8 Modified ROTO embedding

\begin{tabular}{|c|c|c|}
\hline Reagent & Time & Temperature \\
\hline $0.1 \mathrm{M}$ PB & $3 \times 15 \min$ & $4^{\circ} \mathrm{C}$ \\
\hline $\mathrm{OsO}_{4} 2 \%+\mathrm{KFECN}$ & $3 \mathrm{~h}$ & $4^{\circ} \mathrm{C}$ \\
\hline $\mathrm{ddH}_{2} \mathrm{O}$ & $3 \times 15$ min & $4^{\circ} \mathrm{C}$ \\
\hline thiocarbohydrazide & $1 \mathrm{~h}$ & RT \\
\hline $\mathrm{ddH}_{2} \mathrm{O}$ & $15 \mathrm{~min}$ & RT \\
\hline $\mathrm{OsO}_{4} 2 \%$ & $1.5 \mathrm{~h}$ & RT \\
\hline $\mathrm{ddH}_{2} \mathrm{O}$ & $15 \mathrm{~min}$ & RT \\
\hline $2 \%$ uranylacetate & over night & $4^{\circ} \mathrm{C}$ \\
\hline- & & \\
\hline $\mathrm{ddH}_{2} \mathrm{O}$ & $3 \times 15 \min$ & RT \\
\hline $30 \%$ acetone & $15 \mathrm{~min}$ & RT \\
\hline $50 \%$ acetone & $15 \mathrm{~min}$ & RT \\
\hline $75 \%$ acetone & $15 \mathrm{~min}$ & RT \\
\hline $90 \%$ acetone & $15 \mathrm{~min}$ & RT \\
\hline $100 \%$ acetone & $45 \mathrm{~min}$ & RT \\
\hline $25 \%$ Durcupan in acetone & $2 \mathrm{~h}$ & RT \\
\hline $50 \%$ Durcupan in acetone & $2 \mathrm{~h}$ & $\mathrm{RT}$ \\
\hline $75 \%$ Durcupan in acetone & $2 \mathrm{~h}$ & RT \\
\hline $100 \%$ Durcupan in acetone & over night & RT \\
\hline $\begin{array}{l}100 \% \text { Durcupan in acetone + } \\
\text { accelerator }\end{array}$ & $5 \mathrm{~h}$ & RT \\
\hline
\end{tabular}

After Durcupan infiltration optic nerves were transferred into embedding molds filled with Durcupan and polymerized for $48 \mathrm{~h}$ at $60^{\circ} \mathrm{C}$. 


\subsubsection{High pressure freezing and Automated freeze substitution}

High pressure freezing (HPF) is a state-of-the-art method of electron microscopy sample preparation that uses high pressure and rapid cooling with liquid nitrogen. It allows the quasi instantaneous cryo immobilization of tissue which results in structural preservation that is very close to the natural state (Vanhecke et al., 2008) During freezing the water within the tissue turns into amorphous ice without forming crystals that would damage the ultrastructure. During freeze substitution the ice in the sample is exchanged with acetone while fixing and contrasting the samples at low temperatures. This exchange at very low temperatures avoids dehydration and degradation artefacts often found in conventionally fixed tissue (Korogod et al., 2015) and yields excellent structural preservation.

Successful High pressure freezing requires specism to be $200 \mu \mathrm{m}$ or thinner to allow high quality cryo fixation. To achieve this, mice are sacrificed by cervical dislocation and the tissue of interest is immediately collected. To obtain high pressure freezing samples of the optic nerve it was quickly separated from the brain and immersed in ice-cold PBS as preparation for freezing. One arm of the optic nerve was cut into two $1.5 \mathrm{~mm}$ pieces for HPF. For corpus callosum samples one brain hemisphere was immersed in ice cold PBS and $200 \mu \mathrm{m}$ thick sagittal sections were obtained using a vibratome (VT1200S, Leica, Vienna, Austria). A biopsy punch (Harries Unicore $1.5 \mathrm{~mm}$, GE Healthcare, United Kingdom) was used to extract a region of interest in the rostral or caudal corpus callosum of a medial vibratome slice.

The respective sample was then transferred into a drop of $20 \%[\mathrm{w} / \mathrm{v}]$ PVP $(20 \%$ Polyvinylpyrrolidon, Sigma) in PBS and carefully positioned in the HPF A carrier (specimen carrier, Type A and B, (Leica, Vienna, Austria)) avoiding artefacts like tearing. Excessive liquid is removed with a Whatmann filter paper. A carrier B was wetted with hexadecene and added. The resulting carrier sandwich was inserted into a plastic carrier and frozen using a HPM100 high pressure freezer (Leica, Vienna, Austria). The frozen samples were transferred to cryovials and stored in liquid nitrogen until automated freeze substitution (AFS). AFS was carried out as described using a Leica AFS 2. Samples were substituted using 0.1\% Tannic acid (Sigma Aldrich, Munich, Germany) in glass-distilled acetone (Science Services, Munich, Germany) and 2\% osmium tetroxide (Sciences Services, Munich,Germany) (Möbius et al., 2010). 


\begin{tabular}{lll} 
Reagent & Time & Temperature \\
\hline $0.1 \%$ tannic acid in glass distilled acetone & $24 \mathrm{~h}$ & $-90^{\circ} \mathrm{C}$ \\
$-90^{\circ} \mathrm{C}$ cold acetone (pre cooled in the AFS) & $4 \times 30 \mathrm{~min}$ & $-90^{\circ} \mathrm{C}$ \\
$2 \% \mathrm{OsO}_{4}+0.1 \%$ uranylacetate in acetone & $7 \mathrm{~h}$ & $-90^{\circ} \mathrm{C}$ \\
$2 \% \mathrm{OsO}_{4}+0.1 \%$ uranylacetate in acetone & $14 \mathrm{~h}$ & $\begin{array}{l}\text { warm up to }-20^{\circ} \mathrm{C} \\
\text { in } 5^{\circ} \mathrm{C} \text { steps per } \mathrm{h}\end{array}$ \\
$2 \% \mathrm{OsO}_{4}+0.1 \%$ uranylacetate in acetone & $16 \mathrm{~h}$ & $-20^{\circ} \mathrm{C}$ \\
$2 \% \mathrm{OsO}_{4}+0.1 \%$ uranylacetate in acetone & $2.4 \mathrm{~h}$ & warm up to $4^{\circ} \mathrm{C}$ in \\
& & $10^{\circ} \mathrm{C} \mathrm{per} \mathrm{h} \mathrm{increment}$ \\
$2 \% \mathrm{OsO}_{4}+0.1 \%$ uranylacetate in acetone & $1 \mathrm{~h}$ & $4^{\circ} \mathrm{C}$ \\
Temperature matched glass distilled acetone & $4 \times 30 \mathrm{~min}$ & $4^{\circ} \mathrm{C}$ \\
Temperature matched glass distilled acetone & $1 \mathrm{~h}$ & $\mathrm{RT}$ \\
$33 \%$ Epon in Acetone & $2 \mathrm{~h}$ & $\mathrm{RT}$ \\
$50 \%$ Epon in Acetone & $2 \mathrm{~h}$ & $\mathrm{RT}$ \\
$66 \%$ Epon in Acetone & $2 \mathrm{~h}$ & $\mathrm{RT}$ \\
$90 \%$ Epon in Acetone & over night & $\mathrm{RT}$ \\
$100 \%$ Epon & $4 \mathrm{~h}$ & $\mathrm{RT}$
\end{tabular}

Following freeze substitution, the samples were removed from the sample carrier and transferred into embedding molds filled with Epon. Blocks were polymerized at $60^{\circ} \mathrm{C}$ for $24 \mathrm{~h}$.

\subsubsection{Fixation for immunoelectron microscopy (IEM)}

For immunoelectron microscopy (IEM) optic nerves were collected fresh and immersion fixed in Immuno-Karlsson-Schultz fixative overnight at $4^{\circ} \mathrm{C}$ and then stored in $1 \%[\mathrm{w} / \mathrm{v}] \mathrm{Pfa}$ in $0.1 \mathrm{M}$ PB. 


\subsubsection{Tokuyashu sectioning and Immuno electron microscopy}

To visualize the ultrastructural localization of myelin proteins in the optic nerve cyroimmuno electron microscopy on cryosections was performed as described previously (Werner et al., 2007; Möbius et al., 2010). Optic nerves were dissected and immersion fixed in immuno Karlsson-Schultz fixative overnight. Nerves were then cut in 1-2 mm short pieces, stained with toluidine blue, embedded in $10 \%$ gelatin as cubes and infiltrated overnight in $2.3 \mathrm{M}$ sucrose in $0.1 \mathrm{M} \mathrm{PB}$ as cryoprotectant. The cubes were then mounted onto aluminum pins for cryo-sectioning, and frozen in liquid nitrogen. Ultrathin 50-80 nm Tokuyashu sections were cut with a DiATOME Diamond knife, cryo-immuno 2,0 mm (Diatome, Biel, Switzerland) using a Leica UC6 ultramicrotome with a FC6 cryochamber (Leica, Vienna, Austria).The sections were picked up in a 1 to 1 mixture of $2 \%$ methylcellulose and 2.3 M sucrose using a perfect loop (Diatome, Biel, Switzerland), transferred to carbon coated EM grids and stored at $4^{\circ} \mathrm{C}$ (Liou et al., 1996). For labeling, EM grids with sections were placed on PBS droplets and washed $3 \times 2$ min to remove the pickup-solution followed by $3 \times 2$ min washing steps with $0,1 \%$ glycine (Serva, Heidelberg, Germany) in PBS to quench aldehydes. Sections were then blocked for 3 min using 1\% BSA in PBS and incubated with rabbit anti PLP (1:250 A431, custom made K.A.-Nave) or rabbit anti MBP (1:250, custom made (Kathrin Kusch), 1:250) in 1\% BSA in PBS for $1 \mathrm{~h}$. Sections were then washed in $0.1 \%$ BSA in PBS for $5 \times 2$ min followed by 30 min incubation with protein A-gold 1:50 (10 nm, (CMC, Utrecht) in 1\% BSA in PBS. Sections were washed $5 \mathrm{x}$ 2 min with PBS followed by fixation of antibodies by $5 \mathrm{~min} 1 \%$ glutaraldehyde in PBS incubation.

To reduce phosphate that could interfere with contrasting the sections were washed $10 \mathrm{x}$ $1 \mathrm{~min}$ in $\mathrm{ddH}_{2} \mathrm{O}$ and contrasted for $5 \mathrm{~min}$ in neutral uranyl acetate $(2 \%$ in $1.5 \mathrm{~m}$ oxalic acid, $\mathrm{pH}$ adjusted to 7.0 with ammonium hydroxide). For embedding grids were transferred to $1.8 \%$ methylcellulose containing $0.4 \%$ uranyl acetate on ice, incubated for $5 \mathrm{~min}$, and picked up with a custom-made wire loop. Excess liquid was removed from the loop via Whatman filter paper and grids were air-dried.

\subsubsection{Ultrathin sectioning and Electron microscopy}

Epon or Durcupan embedded tissues were cut using a Leica UC7 ultramicrotome (Leica, Vienna, Austria) and a DiATOME diamond knife, ultra $35^{\circ} 3,0 \mathrm{~mm}$. (Diatome, Biel, Switzerland). Blocks were trimmed and $500 \mathrm{~nm}$ semi-thin sections were cut and transferred to glass slides. Semi-thin sections were then stained with methylene blue / Azure II blue for 30-45 sec to visualize lipid rich areas in the tissue to confirm the region of interest. Ultrathin sections of 50-60 nm were cut and transferred to copper mesh grids (Science Services, Munich, Germany) and air dried. The section were contrasted with Uranyless for 30 min (Electron Microscopy Sciences, Hatfield, U.S.A), washed 5 times with $\mathrm{ddH}_{2} \mathrm{O}$ and dried for 30 min. Sections were analyzed with a LEO EM912 Omega (Zeiss, Oberkochen, Germany), and digital electron micrographs were obtained with a wide-angle dual speed 2K-CCD-camera (TRS, Moorenweis, Germany) using the microscope software ImageSP (Sys-Prog, Minsk, Belarus) or iTem (Olympus,Tokyo, Japan). 


\subsubsection{Focussed ion beam scanning electron microscopy}

Focussed ion beam scanning electron microscopy (FIB-SEM) allows to visualize the ultrastructure of tissue not only as 2D sections but as 3D volumes. This enables the analysis of ultrastructural morphology of three-dimensional myelin structures like outfoldings or myelin channels. 3D analysis of this kind was previously possible by serial sectioning but very labor intensive or limited to very small volumes by using tomography. Answering these questions is now becoming more feasible and distinctly faster using FIB-SEM.

To analyze the loss of myelin basic protein in 3D, optic nerves were prepared for electron microscopy by ROTO embedding (see 2.2.9.3) or HPF (see 2.2.9.4) and prepared for volume acquisition in the FIB-SEM. After infiltration in Durcupan optic nerves were minimal embedded by removing as much resin as possible. To achieve this, nerves were cut in 1-2 $\mathrm{mm}$ long pieces and moved on a piece of aclar (ACLAR® Fluoropolymer, Science Services, Munich) until no visible Durcupan layer was left on the nerve (Schieber et al., 2017). This technique allows easy targeting and faster sample preparation of a region of interest (ROI) inside the FIB-SEM. The minimal embedded nerves were polymerized on aclar for $72 \mathrm{~h}$ at $60^{\circ} \mathrm{C}$ and mounted on SEM stubs (12.7x3.1 mm Science Services, Munich, Germany) using conductive silver epoxy (Electron Microscopy Sciences, Hatfield, U.S.A), covered in silver paint (Plano, Wetzlar, Germany) and polymerized overnight at $60^{\circ} \mathrm{C}$. Samples were then sputter coated (Leica Ace 600, Vienna, Austria) with 5-8 nm platinum to limit charging and destruction of the surface during milling and imaging. The samples were then transferred into a Zeiss Crossbeam 540 (Zeiss, Oberkochen, Germany) and stored at vacuum overnight. On the next day the sample was positioned at an angle $54^{\circ}$ to allow orthogonal cutting. Using the software SmartFIB (Zeiss, Oberkochen, Germany) a platinum pad of about $500 \mathrm{~nm}$ was deposited on the area of interest using the FIB at $3 \mathrm{nA}$ current. This is done to protect the surface of the sample and support even milling. A coarse trench was milled to access the region of interest using a $15 \mathrm{nA}$ FIB milling beam followed by polishing of the block face with $7 \mathrm{nA}$. For imaging, the FIB was used at $1.5 \mathrm{nA}$ with a destined milling depth of $30 \mu \mathrm{m}$. FIB milling and SEM imaging were performed simultaneously (Narayan et al., 2014) and images were acquired at $1.5 \mathrm{kV}$ using the software Atlas 5 (Atlas 3D package, Fibics, Ottawa, Canada). For the acquisition an energy selective backscattered (EsB) detector was used at a grid voltage of $1100 \mathrm{~V}$, analytical mode $1000 \mathrm{pA}$. Keyframes were acquired every $400 \mathrm{~nm}$ at $10 \mathrm{~nm}$ xy pixel size and a pixel dwell time of $2 \mu \mathrm{s}$. ROI imaging was performed at 3 or $5 \mathrm{~nm}$ xy pixel size and FIB milling was done every 10 or $50 \mathrm{~nm}$. The total imaging time was set to be less than $2 \mathrm{~min}$. 


\subsubsection{FIB-SEM post processing}

Images were inverted, cropped, aligned (Linear Stack Alignment with SIFT) and smoothed (smoothing function, 3x3 average) using FIJI (Schindelin et al., 2012). The 3D reconstruction was performed manually using Imod (University of Colorado Boulder, Boulder, U.S.A.). Counting of events in image stacks and quantification of object length were done in Microscopy Image browser (Belevich et al., 2016). 


\section{Chapter 3 Results}

\subsection{Generation of an inducible MBP knock-out mouse model}

To investigate the role of myelin maintenance and turnover in the adult CNS a conditional myelin basic protein knock-out mouse line ( $M b p^{f / f f}$, internal line nomenclature MBPFLX) was generated. By selective knock-out of $M b p$ after developmental myelination the MBP potentially required for maintenance of the myelin sheath will be no longer synthesized. The lack of MBP could then be visualized by absence of compact myelin. New myelin membrane formed after knock-out should be MBP negative and not compacted. Mice harbor a floxed Exon 1 of the classical $M b p$ locus and were crossbred to mice heterozygotes for an inducible CreERT2 under the Plp promotor (internal line nomenclature PCET) (Leone et al., 2003) (Figure 1-1, A). At the age of 8 weeks, a time point where myelination is considered mostly complete (Baumann and Pham-Dinh, 2001), mice were injected with $1 \mathrm{mg}$ tamoxifen daily for 10 days with a two day break after 5 days to induce efficient recombination as described for this Cre driver line (Leone et al., 2003). The time point 0 weeks post tamoxifen injection (pti) was defined as the first day after the treatment was completed and mice were analyzed 8 to 52 weeks pti (Figure 3-1, B). Recombination of exon 1 was confirmed on DNA extracted from brain lysate $26 \mathrm{~W}$ pti by polymerase chain reaction (PCR) with primer specific for the site of recombination (Figure 3-1, C).

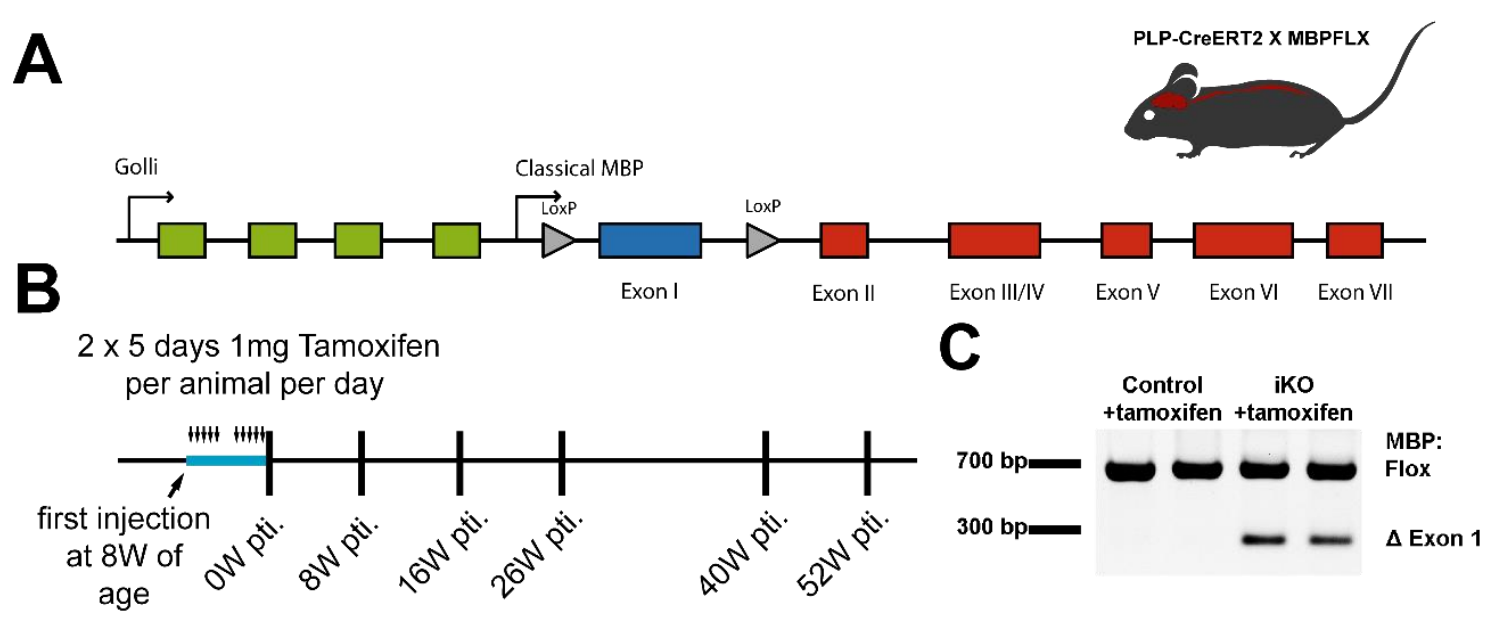

Figure 3-1 Tamoxifen-inducible mouse model of Mbp ablation in adult mice

A) The MBPFLX mouse line was generated from embryonic stem cells obtained from EUCOMM that harbor a floxed Exon 1 of the classical Mbp locus. Mice were crossed to PLP-CreERT2 mice to allow inducible knock-out of MBP in adult oligodendrocytes. B) To induce knock-out of Exon 1, mice were injected for 10 days with $1 \mathrm{mg}$ tamoxifen daily beginning at an age of 8 weeks. C) PCR of brain lysate confirms knock-out of MBP Exon 1 after cre-recombination. 


\subsection{Decrease of Mbp mRNA after knock-out induction}

$M b p$ mRNA is packed into RNA granules and transported to the myelin sheath to be locally translated (White et al., 2008; Torvund-Jensen et al., 2014). Therefore, we expected a longer half-life compared to other myelin genes. To determine the knock-out efficiency, $M b p$ mRNA was quantified in total brain and total spinal cord lysate on 4 time points between 24 hours to 20 days after the first tamoxifen injection of the injection regime (Figure 3-2). Controls

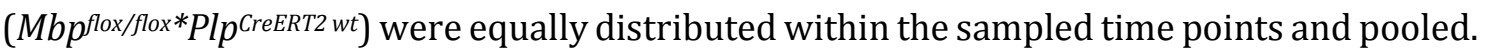
Induced conditional knock-out mice (Mbpflox/flox*PIpCreERT2+, termed iKO) show a tendency towards an increase in mRNA levels $24 \mathrm{~h}$ after the first injection. A similar effect was observed by Koenning and colleagues (Koenning et al., 2012) however the reason for this is unclear. Within 10 days the level of $M b p$ mRNA was decreased to $70 \%$ in brain and $50 \%$ in spinal cord compared to control. 20 days after first injection a reduction to $43 \%$ in brain and $23 \%$ in spinal cord was determined which was similar to expression levels detected at $8 \mathrm{~W}$ with $42 \%$ in brain and $14 \%$ in spinal cord (Figure 3-355, A, B). This indicates that 20 days after the first tamoxifen injection the maximal reduction in $M b p$ mRNA levels was achieved and that the remaining transcripts could originate from non-recombined cells.
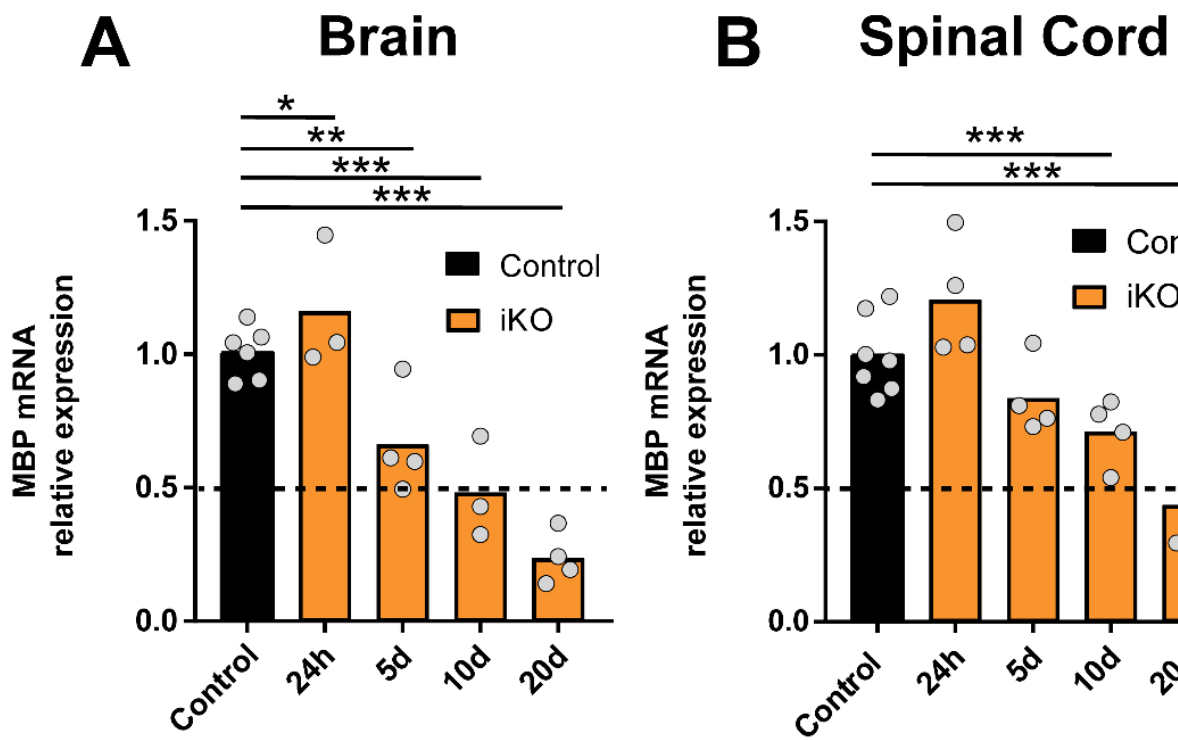

[Time since first tamoxifen injection]

Figure 3-2 MBP mRNA decreases within 20 days after first day of tamoxifen injections

Tamoxifen was injected for 10 days and mice were sacrificed at the indicated time points after first tamoxifen injection. A) mRNA extracted from brain and (B) spinal cord. N=3-4 animals, controls were time point matched and later on pooled. 20 days after first tamoxifen injection the levels of MBP mRNA reached baseline (Figure 3-36) in brain and spinal cord. Each time point was compared to the pooled controls, expression was normalized to Rps13 and Ppia, two-tailed unpaired t-test; p <.05 (*), p <.01 (**), p <.001 (**)) Ctrl= control (Mbp flox/flox);iKO = induced conditional knock-out (Mbp flox/flox*PlpCreERT2) both conditions received tamoxifen. 


\subsection{Ongoing expression of major myelin genes}

To determine whether the expression of other myelin genes is affected by the $M b p$ knockout, qPCR analysis of Golli, Plp1, Mag, Mog and Cnp was performed on brain lysate from animals 8, 16 and 26 weeks pti. None of these myelin gene transcripts were decreased in abundance. Instead, a trend towards increased mRNA abundance was seen in Plp1, Mag, Mog and Cnp at 26 weeks pti (Figure 3-3). Hence, the loss of Mbp expression in adult oligodendrocytes in the brain does not result in reduced expression of the other major myelin proteins.
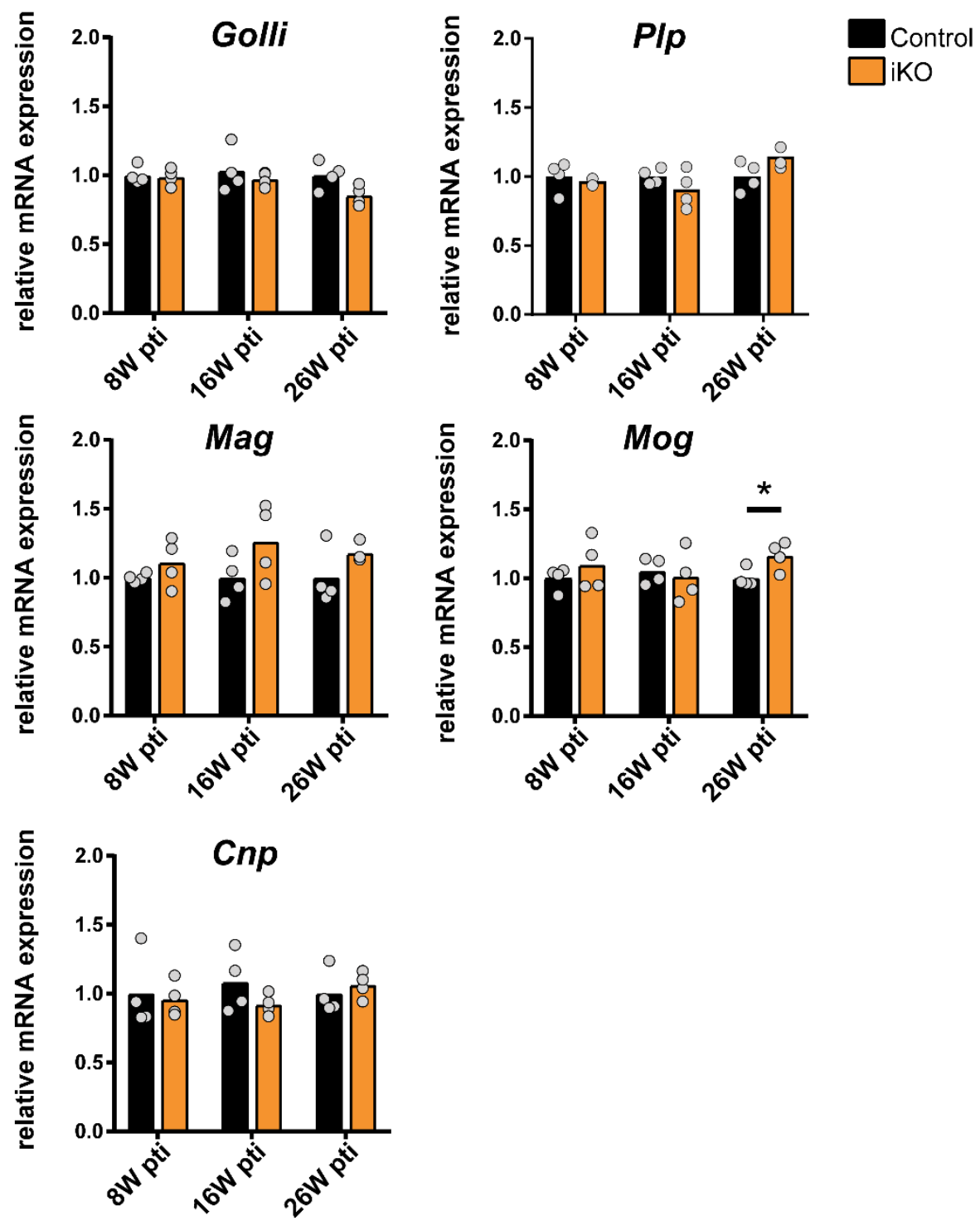

Figure 3-3 The expression of PLP, Golli, MAG, MOG and CNP is not affected after MBP knock-out

Despite knock-out of $\mathrm{Mbp}$ the expression of Golli transcripts, compact ( $\mathrm{Mag}, \mathrm{Mog}$ ) and non-compact (Cnp) myelin genes are not decreased. A tendency for minor increase in mRNA abundance can be seen in Mag, Mog and Cnp at 26W pti. mRNA extracted from total brain lysate, $\mathrm{N}=4$ animals per condition, expression was normalized to the housekeeping genes Rps13 and Ppia, two-tailed unpaired t-test, $\mathrm{p}<.05\left(^{*}\right)$. 


\subsection{Reduced abundance of major myelin proteins}

To address the effects of $M b p$ knock-out on the abundance of myelin proteins, immunoblot analysis was performed on total brain lysate of animal 8, 16 and 26W pti (Figure 3-4). At $8 \mathrm{~W}$ pti (Figure 3-4, A) a reduction in the abundance of MBP by $40 \%$, PLP (20\%) and MOG (35\%) was observed (Figure 3-4, C). A further decrease in abundance at $16 \mathrm{~W}$ pti of MBP by $60 \%$, PLP (40\%), MOG (25\%) and 26W pti of MBP by 75\%, PLP (30\%), MOG (30\%) and MAG (35\%) was found.

Quantification of MBP showed that at $8 \mathrm{~W}$ MBP was reduced to $62 \%$, on $16 \mathrm{~W}$ to $41 \%$ and on $26 \mathrm{~W}$ to $26 \%$. A fitted exponential curve with one phase decay to model protein loss (Lüders et al., 2019) was calculated for the three time points $\left(Y=(0,9669-0,08798) * \mathrm{e}^{-}\right.$ $\left.0,06276^{*} \mathrm{x}\right)+0,08798$ ). Based on this equation the reduction of MBP by $50 \%$ was reached by approximately 11 weeks or 77 days. This is within range of half replacement times for MBP from Fornaserio et al. which ranged from 70 days in brain in a long pulse experiment to 82 days in cortex and 101 days in cerebellum in shorter pulse experiments (Fornasiero et al., 2018). In contrast to MBP the abundance of PLP, MAG and MOG were consistently decreased to about $70-80 \%$ of control at $8 \mathrm{~W}, 16 \mathrm{~W}$ and $26 \mathrm{~W}$ pti.

This shows that MBP is a very stable protein that persists many weeks after its synthesis in mature oligodendrocyte was abolished by the induced knock-out. The concomitant loss of the other compact myelin proteins indicates that knock-out of MBP affects myelin and results in a reduction of the whole compact myelin compartment. Fluorescent immunohistochemistry of MBP on brain sections at 26W pti showed reduced MBP signal in different CNS regions like the corpus callosum, the fimbria, the cerebellum and optic nerve, indicating that the loss of MBP occurs in all observed brain regions (Figure 3-5, $A^{\prime}-C^{\prime}$ ). 


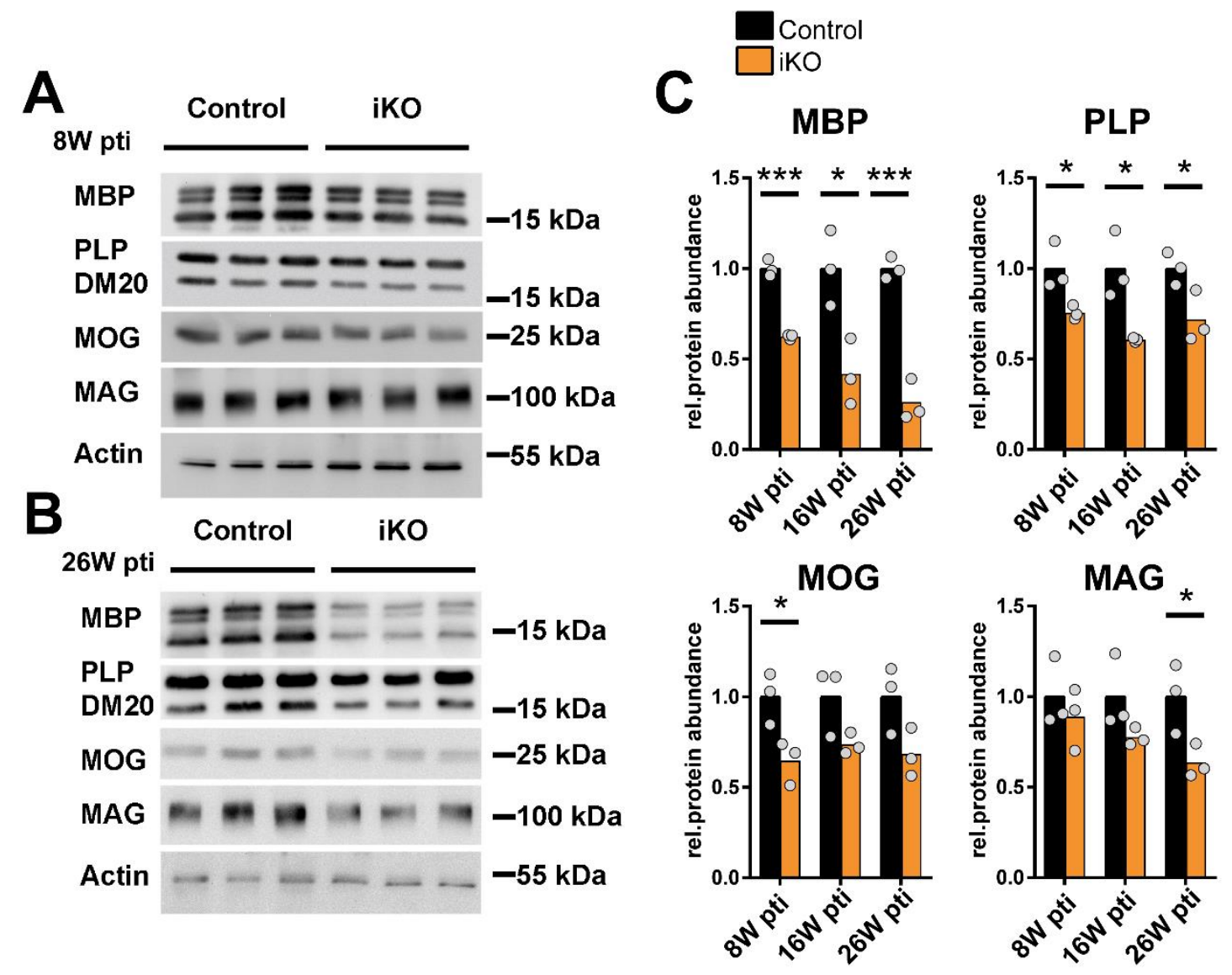

Figure 3-4 Protein abundance of myelin proteins is decreases after MBP knock-out

A-B) Immunoblot analysis of myelin proteins $8 \mathrm{~W}$ pti (A) and $26 \mathrm{~W}$ pti (B) shows a strong reduction in MBP protein abundance after $26 \mathrm{~W}$ pti and to a lesser extent reduction in PLP, MAG and MOG levels. The loss of these myelin proteins is indicative for loss of the compact myelin compartment. Quantification is shown in (C). Total brain lysate, normalized to actin, $\mathrm{N}=3$ animals, two-tailed unpaired t-test; $\mathrm{p}<.05\left(^{*}\right), \mathrm{p}<.01\left(^{* *}\right), \mathrm{p}<.001\left({ }^{* * *}\right)$. 


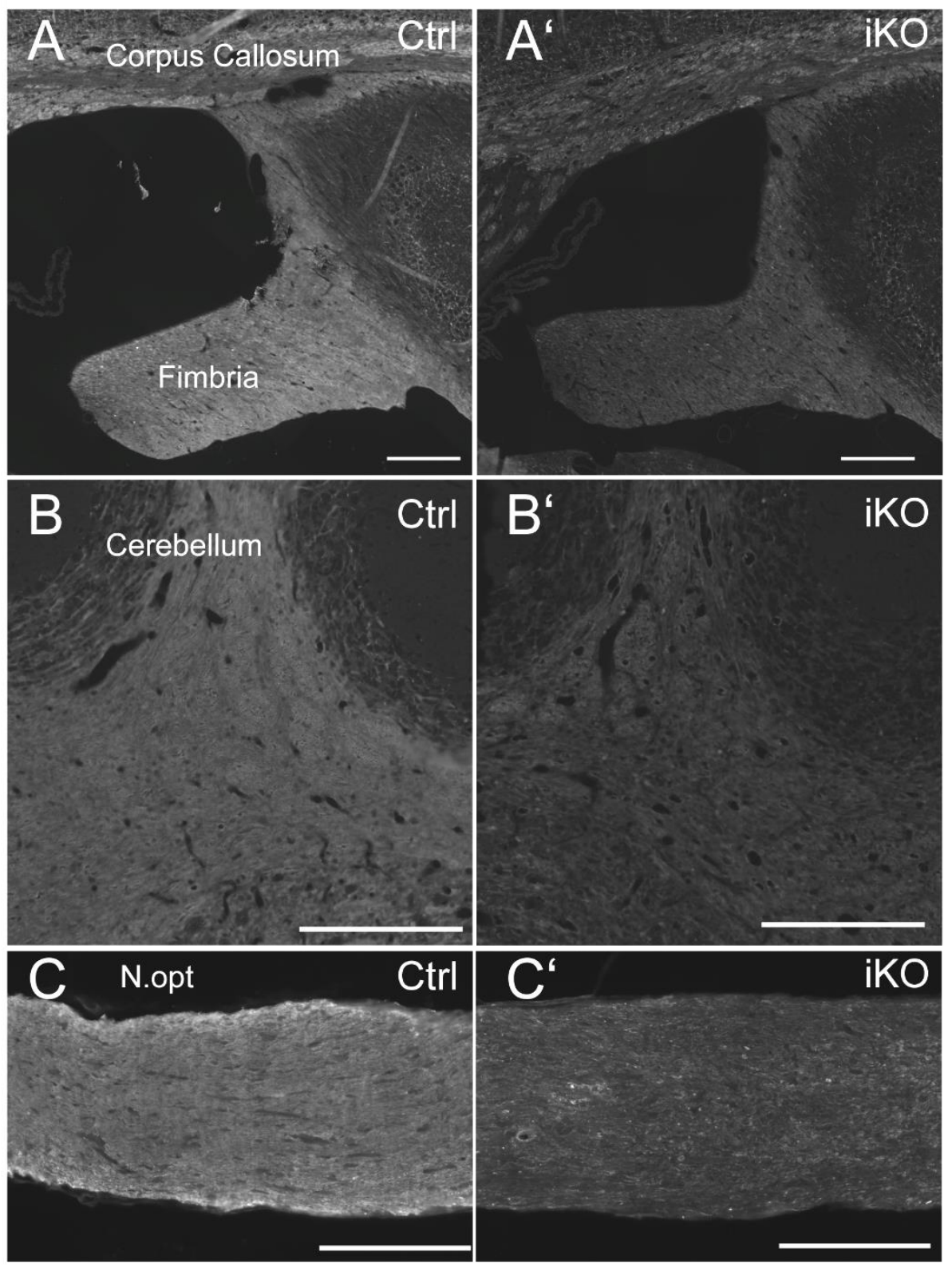

Figure 3-5 Immunofluorescence staining for MBP in brain and optic nerve

Corpus callosum, fimbria (A), cerebellum (B) and optic nerve (C) in control and iKO mice 26W pti were fluorescently labeled for MBP and show less MBP signal in the iKO, scale bar $200 \mu \mathrm{m}$. 


\subsection{Tremor and motor impairments occur months after MBP ablation}

The loss of MBP is accompanied by a slowly progressive development of a motor phenotype in male and female knock-out mice (Figure 3-6). Knock-out mice develop a tremor around $20 \mathrm{~W}$ pti that begins with subtle shivering during movement (tremor score 1), progressively increases between 30 and $35 \mathrm{~W}$ pti towards tremor during resting (tremor score 2) and reaches its maximum with pronounced shivering (tremor score 3) around 40W pti. Later the tremor ceases as the mice age (Figure 3-6, B). The development of the tremor is accompanied by loss of body weight (Figure 3-6, A) and impaired motor functions (Figure $3-4$, C) as quantified by the capability of the mouse to leave a small box placed in the cage (Figure 3-4, D). IKO mice at 26W pti can leave the box with some difficulties due to hind limb weakness and therefore need more time for this task. Moreover, they are not able to jump out of the box like control mice and instead need to climb using their front legs. However, iKO mice are not as severely affected as the MBP mutant shiverer mice which suffer from a severe tremor and die prematurely by seizures within 10-12 weeks of age (Chernoff, 1981) 

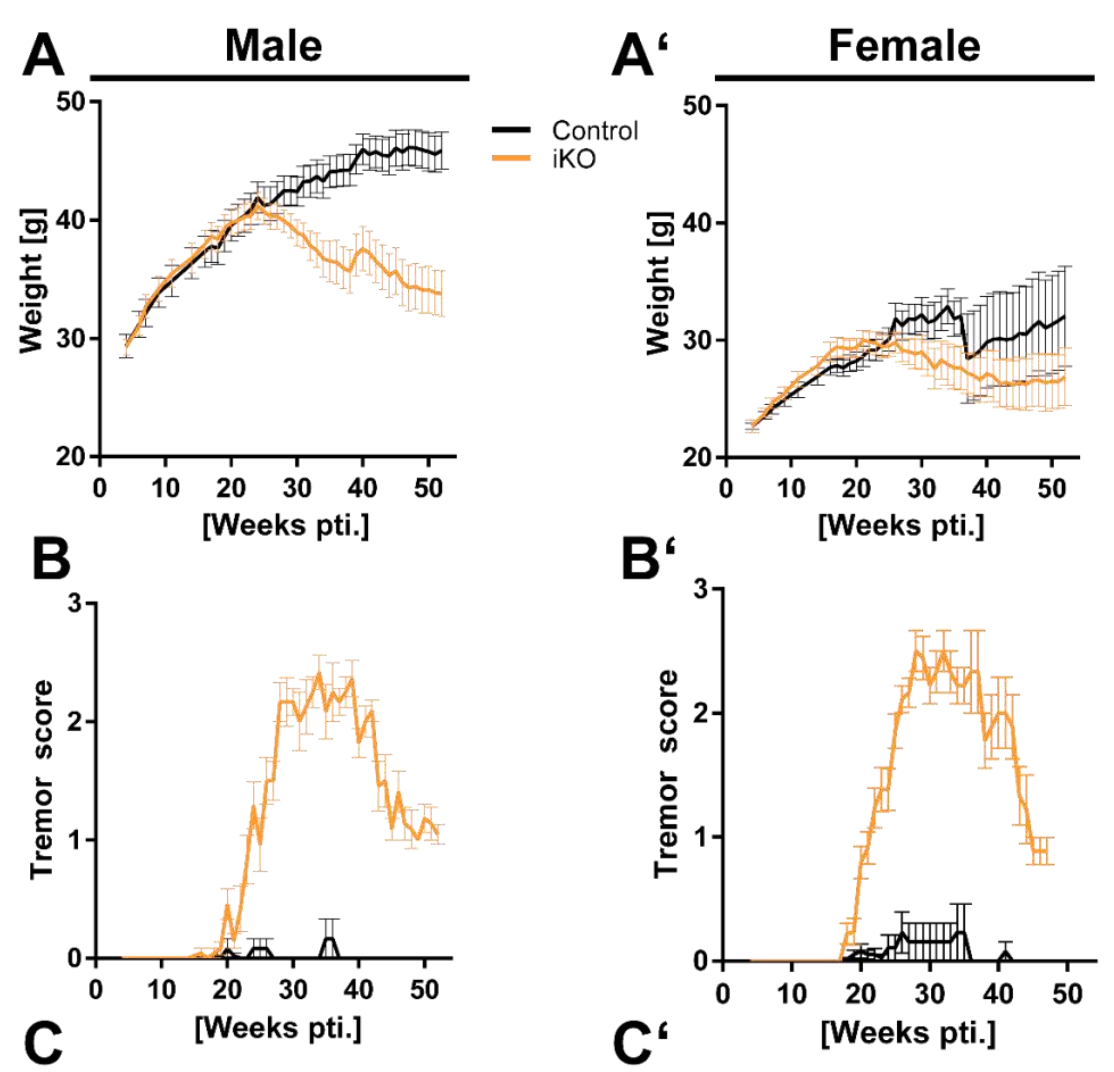

$\mathrm{B}^{\prime}$
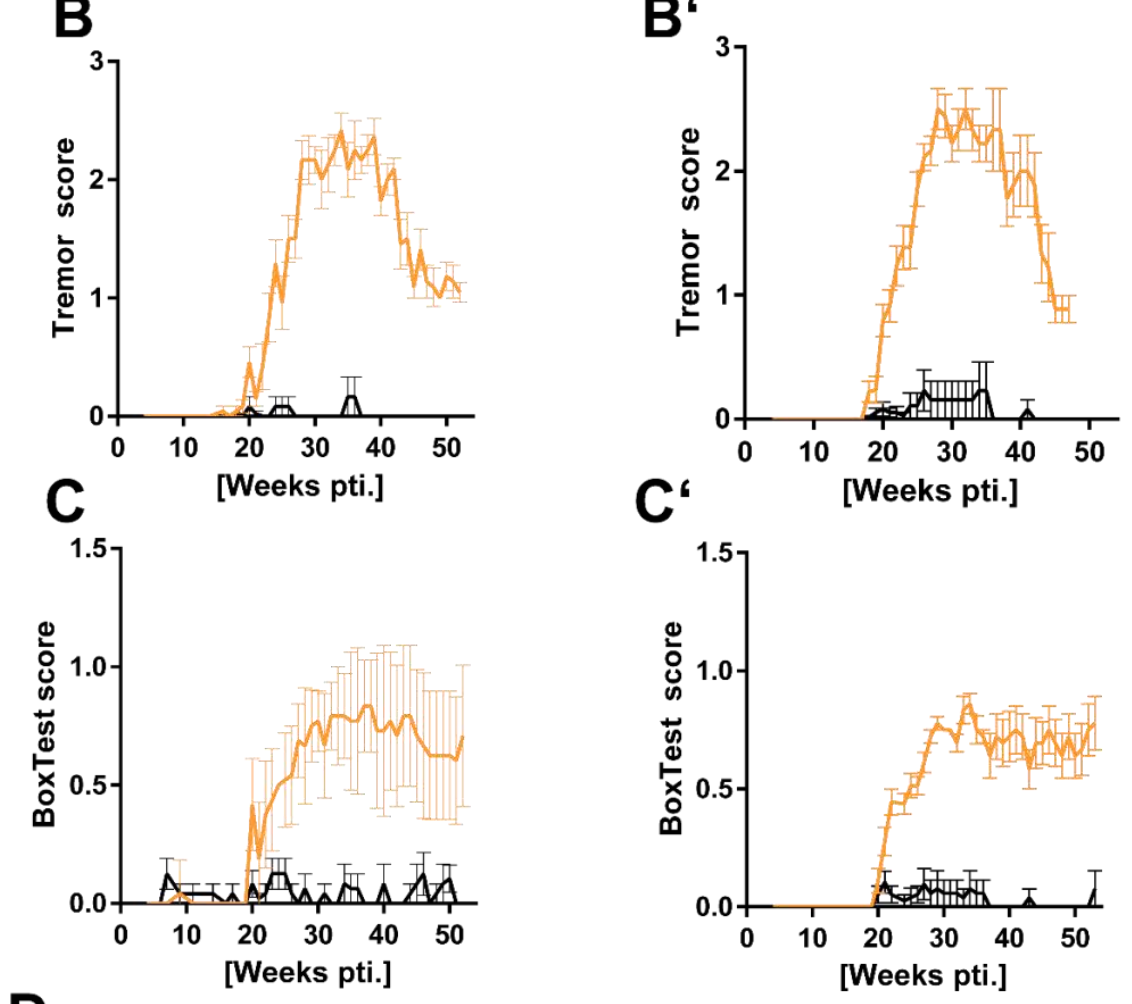

D
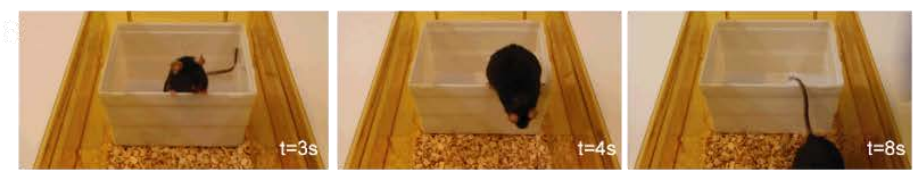

Figure 3-6 Tremor assessment and development of body weight after knockout induction

(A-C')Male and female knock-out mice show normal weight increase until 20-30 weeks pti. After that mice progressively lose weight $\left(A, A^{\prime}\right)$, develop tremor $\left(B, B^{\prime}\right)$ and show difficulties in leaving a plastic box which requires motor coordination and hind limb strength ( $C, C^{\prime}$ and $\left.D, D^{\prime}\right)$. Tremor score: No tremor (0), tremor during movement like walking or climbing (1), tremor during resting (2). Box test: Leaving the box within 3 min with ease (0), leaving the box within 3 min with coordination problems (hanging on and falling over the edge), not leaving the box within $3 \mathrm{~min}$ (1) ( $\mathrm{Ctrl}=\mathrm{control}(\mathrm{Mbp}$ flox/flox); iKO=induced conditional knock-out $\left(M_{b p}\right.$ flox/flox* $^{*}$ Plp $\left.{ }^{\text {CreERT2 }}\right)$ both conditions received tamoxifen. $\mathrm{N}=12$ vs 12 male and 11 vs 11 female mice were scored bi-weekly, error bar represents SEM. 


\subsection{Emergence and origin of non-compact membrane processes at the inner tongue}

The optic nerve is clearly affected by loss of MBP (Figure 3-5, C') and was chosen as model tissue as it is a well-defined highly myelinated CNS white matter tract with the axons running mostly parallel and containing a limited amount of cell types. It can be dissected as intact fresh tissue and processed by HPF for excellent structural preservation or other sample preparation procedures for different analyses. To visualize structural changes of the compact myelin sheath induced by the abolishment of MBP synthesis, optic nerve samples were prepared for fine structural analysis by electron microscopy using high pressure freezing (HPF) and freeze substitution (FS).
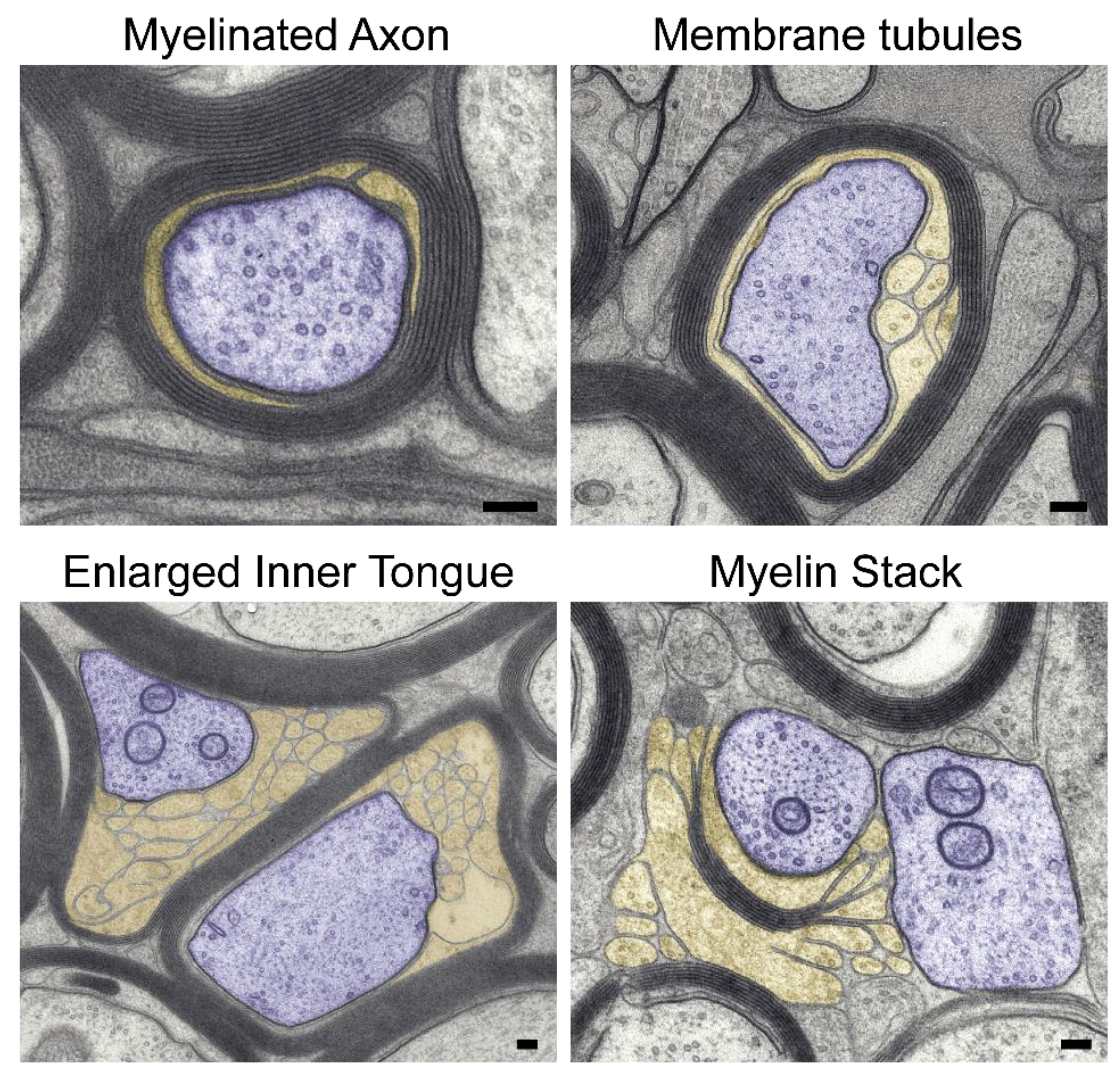

Figure 3-7 Ablation of MBP results in formation of non-compact membrane tubules

Analysis of myelin morphology 16W after MBP knock-out induction on an optic nerve cross section from a region close to the retina. The loss of MBP results in the formation of membrane tubules at the inner tongue. Where most of the compact myelin is lost membrane tubules loop out and form "myelin stacks" leaving parts of the axon demyelinated. The non-compact myelin tubules can be seen disconnected from compact myelin in the vicinity of myelinated and non-myelinated axons (purple: axon, orange: membrane tubules). TEM micrographs of high pressure frozen (HPF) freeze substituted (FS) optic nerve 16W pti, scale bar $100 \mathrm{~nm}$. 
When analyzing optic nerves $16 \mathrm{~W}$ pti the following structural changes could be observed: At the adaxonal myelin site membrane structures emerge that massively enlarge the inner tongue (Figure 3-7). Occasionally, remnants of short myelin stretches with loop-like tubulations can be observed, which we called myelin-stacks (Figure 3-7, Myelin stack) that lay in close apposition to the axon covering it incompletely. These membrane processes have striking similarity to non-compact membrane processes seen in dysmyelinated tracks of shiverer mice (Figure 1-3) suggesting that they are oligodendrocyte derived membrane.
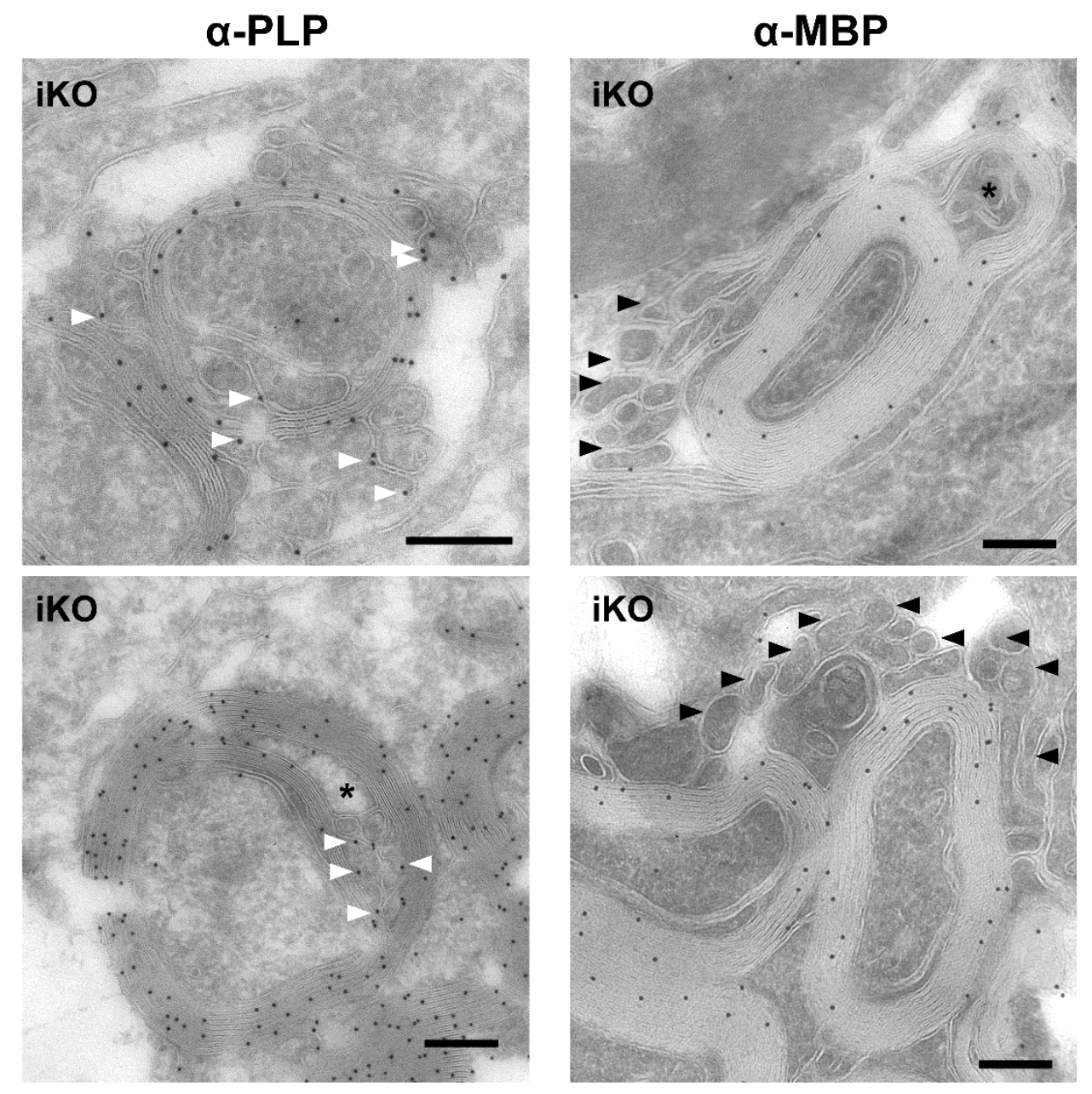

Figure 3-8 Myelin tubulations are oligodendrocyte-derived membrane

Membrane tubules found at the inner tongue and in the surrounding of axons of MBP iKO mice are PLP positive (white arrow head) but lack MBP labeling (black arrow head) suggesting an oligodendrocytic origin. Optic nerve at $26 \mathrm{~W}$ pti, $10 \mathrm{~nm}$ protein-A gold particles bound to primary antibody against PLP (custom made antibody A431) and MBP (Custom made antibody, Dr. Kathrin Kusch), scale bar $200 \mathrm{~nm}$.

To investigate whether these emerging membrane processes are myelin derived, immunogold labeling of the major myelin proteins MBP and PLP was performed. The membrane processes as well as the compact myelin are PLP positive as indicated by $10 \mathrm{~nm}$ gold particles (Figure 3-8, white arrowhead). The MBP labeling reveals that the compact myelin is clearly MBP positive, the observed membrane tubules at the inner tongue and also outside the axon are MBP negative (Figure 3-7, black arrow heads). From the clear presence of the myelin marker PLP it can be concluded that the membrane tubules are myelin-related membranes derived from oligodendrocytes. The absence of MBP indicates that this membrane is either aged myelin where MBP has been removed or myelin membrane that never contained MBP since it was newly synthesized after MBP ablation. 


\section{Tubules at inner tongue}

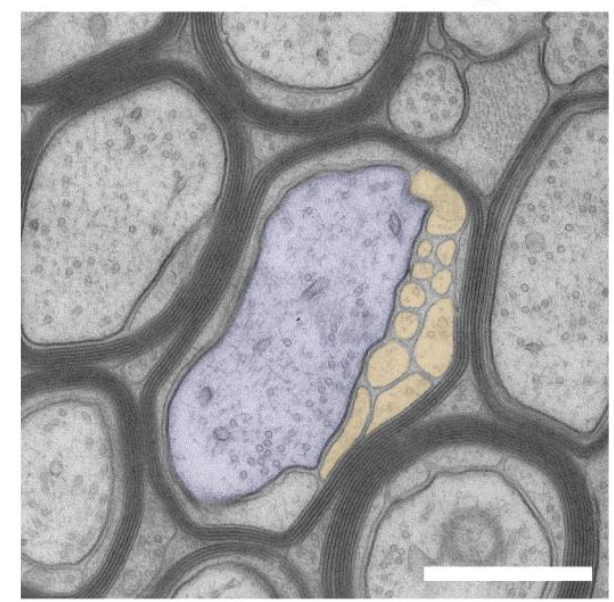

Inner and outer tongue

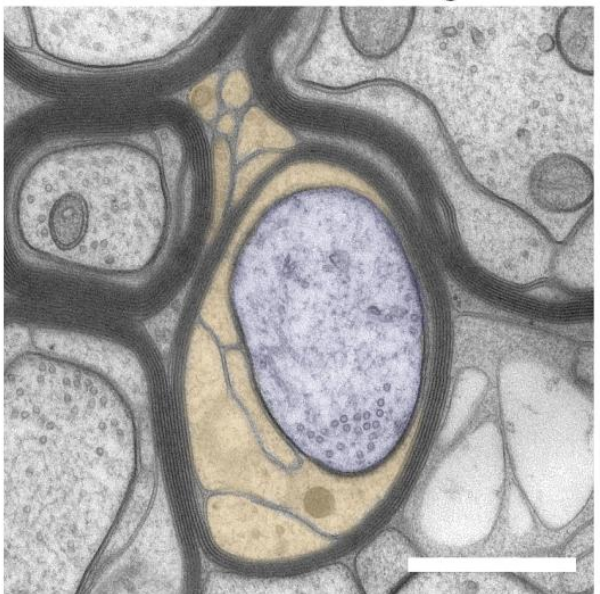

\section{Tubules adjacent to axon}
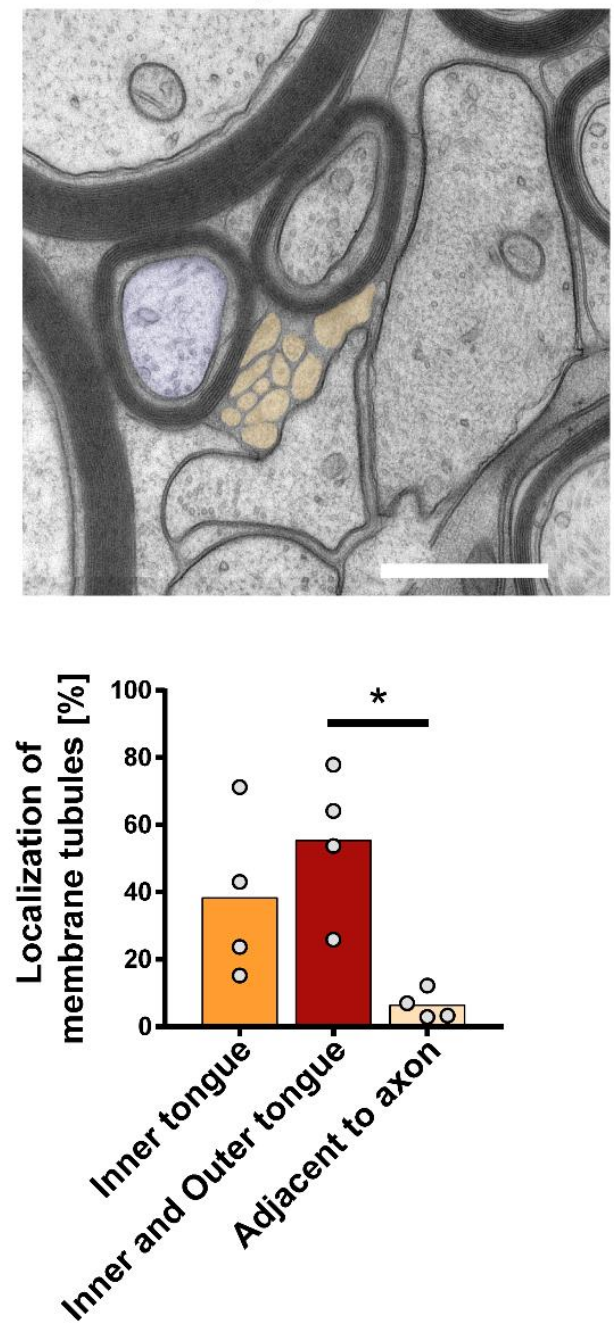

Figure 3-9 Membrane tubules occur predominantly at the inner tongue

Non-compact membrane tubules can be observed first at the inner tongue of the oligodendrocyte in MBP iKO mice. Quantification of tubule localization reveals that tubulation in the outer tongue is mostly associated with tubulation at the inner tongue. High pressure frozen and freeze substituted optic nerve, $16 \mathrm{~W}$ pti. $\mathrm{N}=4 \mathrm{iKO}$ animal with at least 4 random sampled electron micrographs, One-Way Anova with Tukey's multiple comparisons test, scale bar $500 \mathrm{~nm}$. 
To assess whether the observed phenotype first occurs at the inner or the outer tongue the occurrence of non-compact myelin membrane tubules was assigned to either inner tongue, outer tongue or both locations and quantified in high pressure frozen optic nerve at $16 \mathrm{~W}$ pti (Figure 3-9). This assessment showed that myelin membrane tubules occur mostly at the inner tongue. Some tubules were localized adjacent to the axon but due to limitations of the 2D image representation those are indistinguishable from the outer tongue. In many cases myelinated profiles showed myelin tubules at the inner tongue and similar membrane structures outside of the myelin sheath (termed inner and outer tongue) (Figure 3-9). Since outer tongue membrane tubules have not been observed independently of an inner tongue phenotype, we consider the inner tongue as site of first emergence of these myelin tubules. The observed outer tongue phenotype is considered a secondary effect following the initial emergence of inner tongue membrane structures. 


\subsection{Loss of myelinated profiles and decreased myelin thickness}

To investigate the formation of these observed inner tongue tubules in more detail (Figure 3-7) electron micrographs of high pressure frozen optic nerve samples at $8 \mathrm{~W}, 16 \mathrm{~W}$ and $26 \mathrm{~W}$ pti were analyzed and showed the progressive phenotype of myelin loss, increase in unmyelinated axons as well as phenotypic axons with inner tongue membrane processes or myelin stacks (Figure 3-10, A). Sample preparation by high pressure freezing is an excellent tool for close to native tissue preservation and assessment of myelin phenotypes (Möbius et al., 2016) Here this technique facilitated the identification of the phenotype and differentiation between non-myelinated axons and other cellular processes from astrocytes or microglia (Möbius et al., 2016). In this case HPF material was used to validate that the observed larger round processes are non-myelinated axons.

For statistical analysis chemically fixed material was used to allow a representative sampling over the total cross section of an optic nerve, which is not possible in HPFprepared samples due to freezing artifacts in some areas of the tissue. In micrographs of the optic nerve at $8 \mathrm{~W}$ pti approximately $60 \%$ of all axons per field of view are normally myelinated, $20 \%$ show membrane processes at the inner tongue and $10 \%$ are nonmyelinated. In comparison in control animals approximately $90 \%$ of axons are normally myelinated and 7\% are non-myelinated (Figure 3-10, B). The number of myelinated fibers further decreases at $16 \mathrm{~W}$ and $26 \mathrm{~W}$ pti with a concomitant increase in the number of nonmyelinated fibers. The percentage of axons that show a phenotype including enlarged membrane tubules at the inner tongue or presence of myelin stacks deceased from approximately $25 \%$ ( $8 \mathrm{~W}$ pti) to $5 \%(52 \mathrm{~W}$ pti). Interestingly, axonal loss was not observed in any analyzed time point (Figure 3-10, B).

Loss of myelin was also observed in other myelinated tracts of the CNS. At 26W pti the density of myelinated fibers was decreased in optic nerve, caudal corpus callosum (cCC) and the corticospinal tract of spinal cord (SC CST). The relative reduction of myelinated fiber density was highest in optic nerve (55\% reduction) followed by SC CST (40\%) and the least affected caudal corpus callosum (30\%) (Figure 3-11, A). When analyzing the progression of the demyelination in the SC CST the myelinated axon density showed a high variation and no significant reduction at $16 \mathrm{~W}$ pti, but a decreased density at $26 \mathrm{~W}$ pti ( $40 \%$ reduction) which was further decreased at $52 \mathrm{~W}$ (70\% reduction) compared to control. 

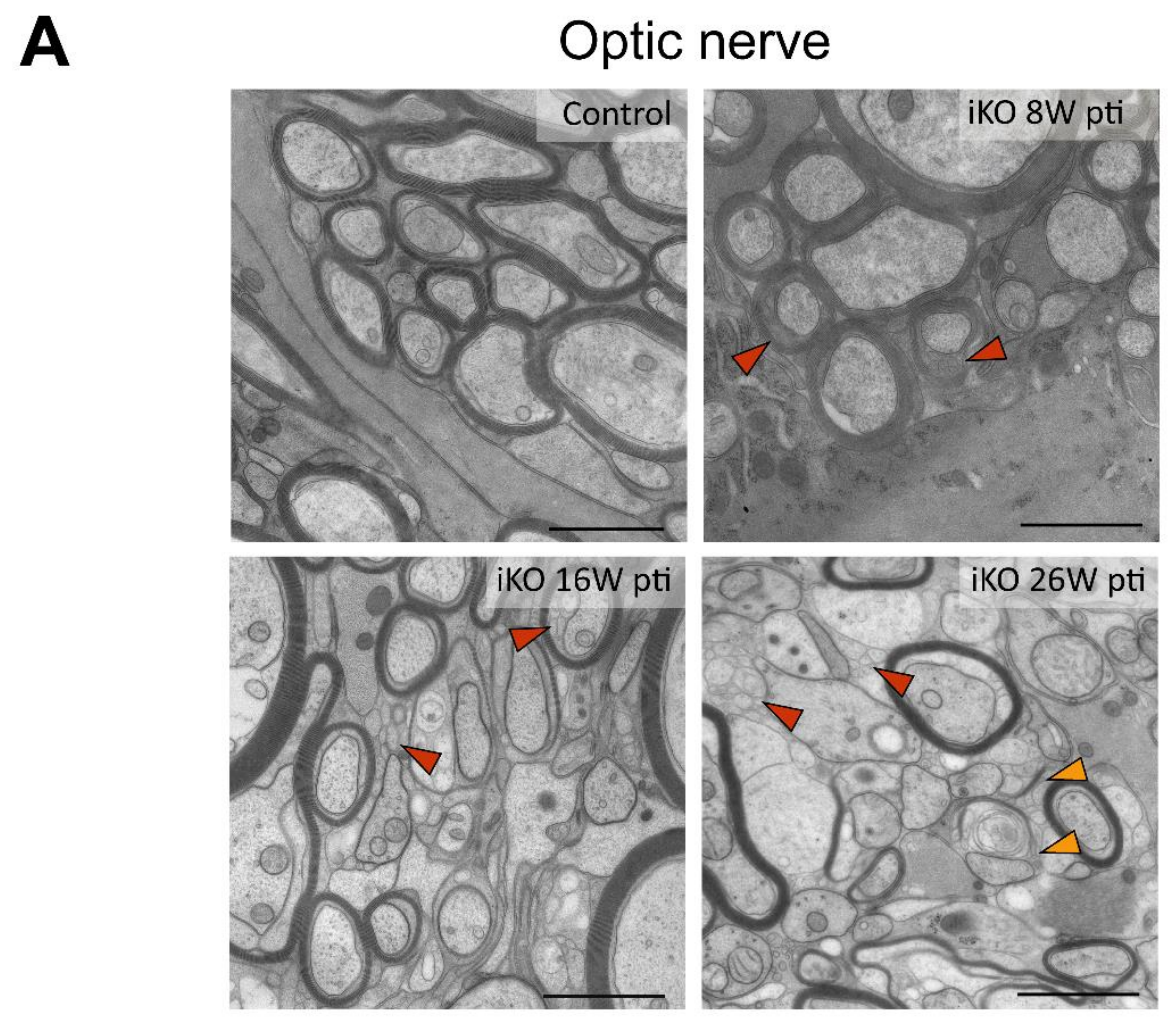

\section{B}

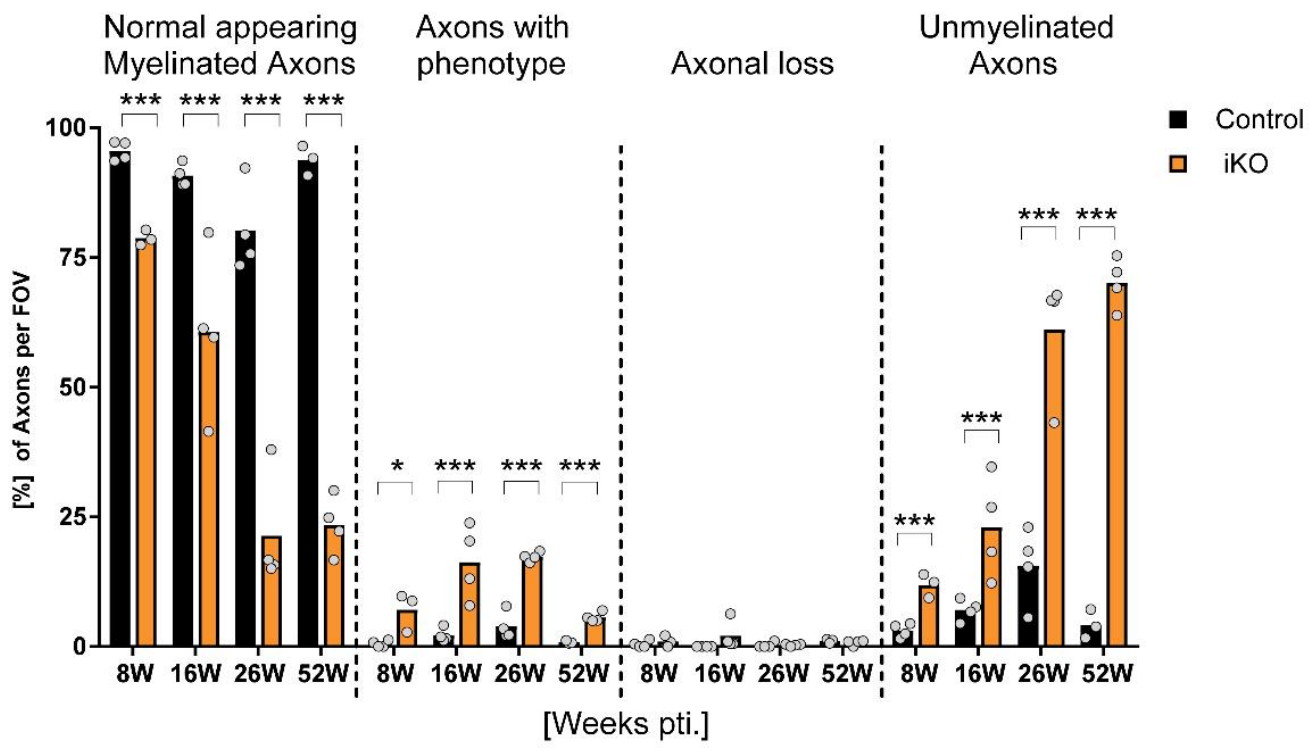

Figure 3-10 Progressive loss of myelinated and increase in unmyelinated axons after ablation of MBP

(A) Transmission electron micrographs of optic nerve (HPF) reveal the emergence of non-compact myelin structures (red arrow head), loss of compact myelin (orange arrow head) and an increase in non-myelinated axons. (B) Quantification of all axons per field of view (FOV) reveal that the percentage of normally myelinated axons is progressively decreasing while axons with phenotypic membrane processes and membrane stacks as well as unmyelinated axons are proportionally increasing. No axonal loss of was observed. Analysis was performed on chemically fixed (K\&S) cross sections in the retinal part of the optic nerve of at least 5 random sampled micrographs with a total area of $>330 \mu \mathrm{m}^{2}$ and $>200$ axons per animal. $\mathrm{N}=3-4$ animals, two-tailed unpaired $t$-test, $p<.05\left(^{*}\right), p<.01\left({ }^{* *}\right), p<.001\left(^{* * *}\right)$ scale bar $1 \mu \mathrm{m}$. 

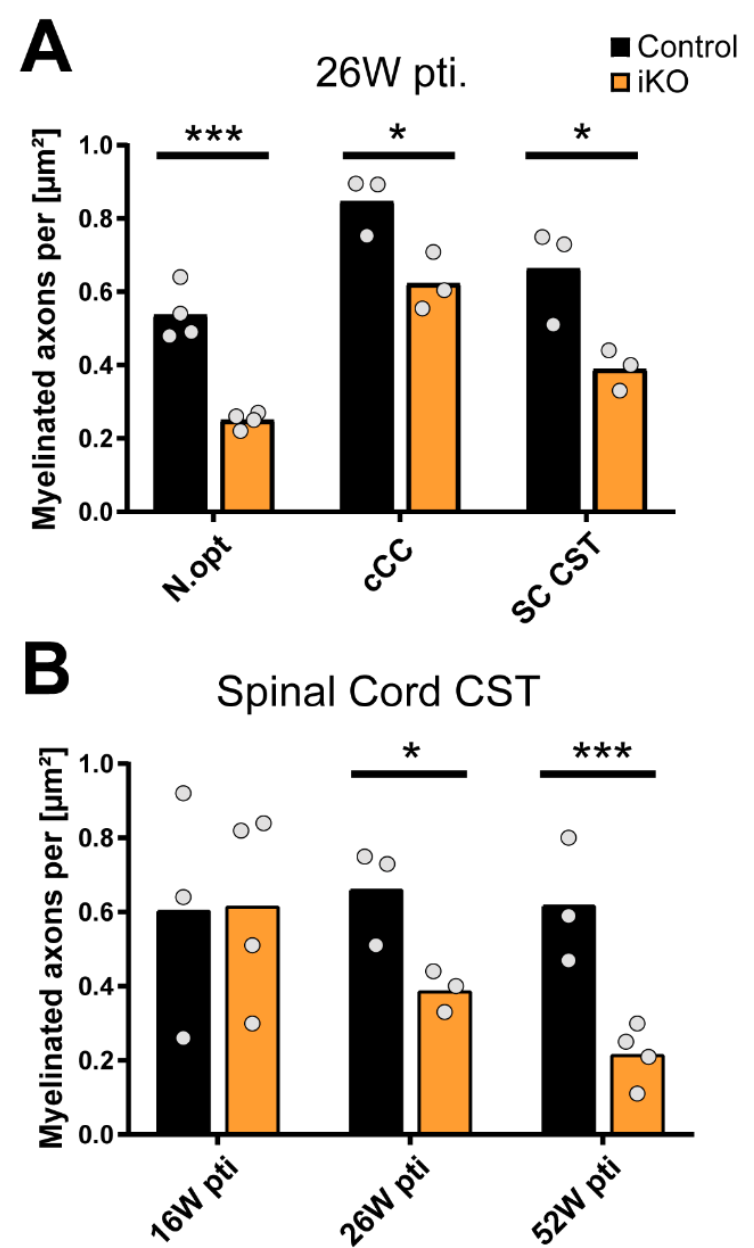

Figure 3-11 Loss of compact myelin is observed in optic nerve, corpus callosum and spinal cord

Decreased density of myelinated axons was observed in different parts of the CNS including optic nerve (n.opt), caudal corpus callosum (cCC) and the corticospinal tract of the spinal cord (CST) (A). Heterogeneity in the loss of myelinated profiles can be observed between regions with the strongest reduction seen in N.opt followed by SC CST and cCC. (B) Density of myelinated axons is decreased in SC CST 26W pti and 52W pti. Analysis was performed on cross sections in the retinal part of the optic nerve, the caudal corpus callosum and the CST of the spinal cord of at least 5 random sampled micrographs with a total area of $>330 \mu \mathrm{m}^{2}$ and $>200$ axons. N=3-4 animals, two-tailed unpaired $t$-test, $p<.05\left(^{*}\right), p<.01\left(^{* *}\right), p<.001(* * *)$.

To asses not only effects of MBP iKO on the number of myelinated profiles, but also on the thickness of the myelin sheath, measurements of a corrected g-ratio were performed. Due to the previously described enlargement of the inner tongue, a conventional g-ratio assessment would result in a skewed representation of compact myelin thickness since inner tongue and compact myelin are usually not considered separately. To avoid this, the area of the inner tongue was subtracted from the complete fiber area before deriving the diameter assuming a circular area. The corrected g-ratio was calculated without the inner tongue by dividing the calculated diameter of the compact myelin by the calculated diameter of the axon. (Figure 3-12, A). 

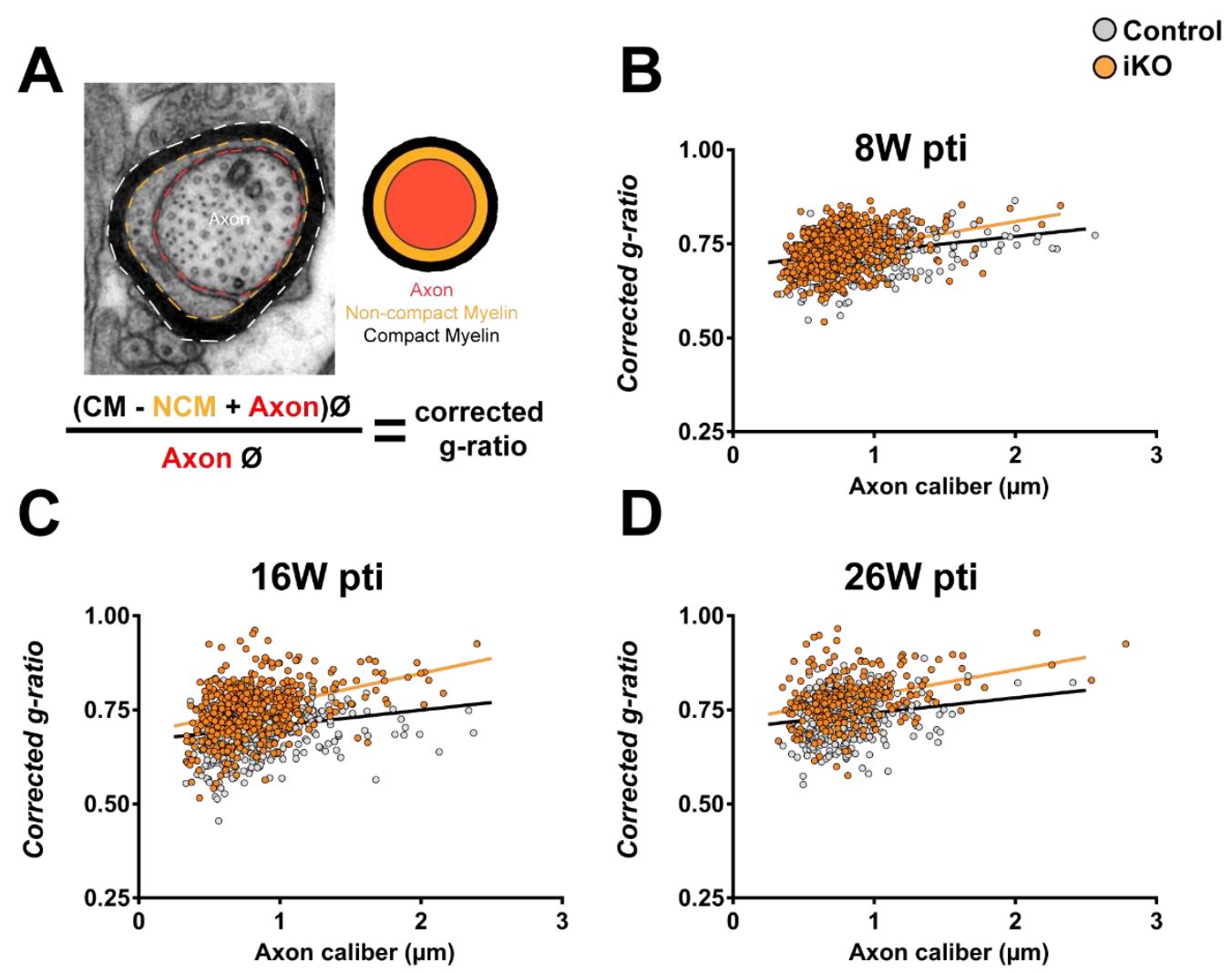

Figure 3-12 Increased corrected g-ratio in optic nerve

Corrected g-ratio in optic nerve 8W pti (A), 16W pti (B) and 26W pti (C) was increased over the whole range of axonal calibers. At least 150 axons per animal and $\mathrm{N}=3-4$ animals were analyzed. Linear regression line (black control, orange iKO) To correct for non-compact myelin enlargement the inner tongue area was subtracted from the total fiber area and a corrected diameter was calculated. The resulting diameter was then divided by the axonal diameter to obtain a corrected g-ratio. Unmyelinated axons were excluded from the analysis.

A cloud upshift for the corrected g-ratio was observed for axons in the optic nerve $8 \mathrm{~W}, 16 \mathrm{~W}$ and 26W pti (Figure 3-12, B-D). This showed that in addition to in the reduced number of myelinated axon profiles a reduction in myelin thickness can be observed after ablation of MBP. In contrast, no reduction in corrected g-ratio has been observed in axons of the spinal cord (Figure 3-12, A) or the corpus callosum (Figure 3-12, B) 26W pti. 

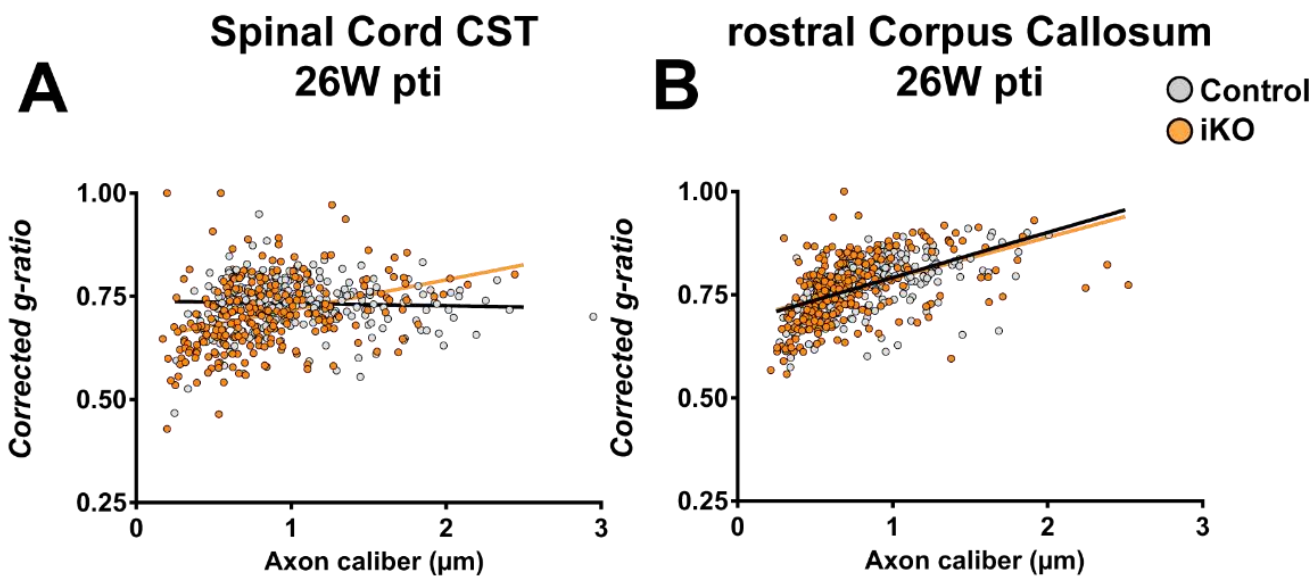

Figure 3-13 Corrected g-ratio in spinal cord and rostral corpus callosum

Corrected g-ratio in CST of spinal cord (A), and rostral corpus callosum (B) 26W pti. In the CST (B) and corpus callosum (B) no change in corrected g-ratio is observed. At least 150 axons per animal and $\mathrm{N}=3-4$ animals were analyzed and a linear regression was plotted (black control, orange iKO). 


\subsection{No obvious ultrastructural changes in PNS and in the absence of tamoxifen}

To test whether the motor phenotype described earlier (Figure 3-6, B,C) is a consequence of demyelination in the peripheral nervous system, electron micrographs of sciatic nerve 16W pti were analyzed. Obvious myelin abnormalities could not be observed. (Figure 3-14) This is in accordance with previously published work showing that shiverer PNS myelin shows minor alterations such as a few layers of uncompact myelin in the inner tongue and an increased number of Schmidt-Lantermann incisures (Rosenbluth, 1980; Gould et al., 1995), but is otherwise functional (Privat et al., 1979; Uschkureit et al., 2000).

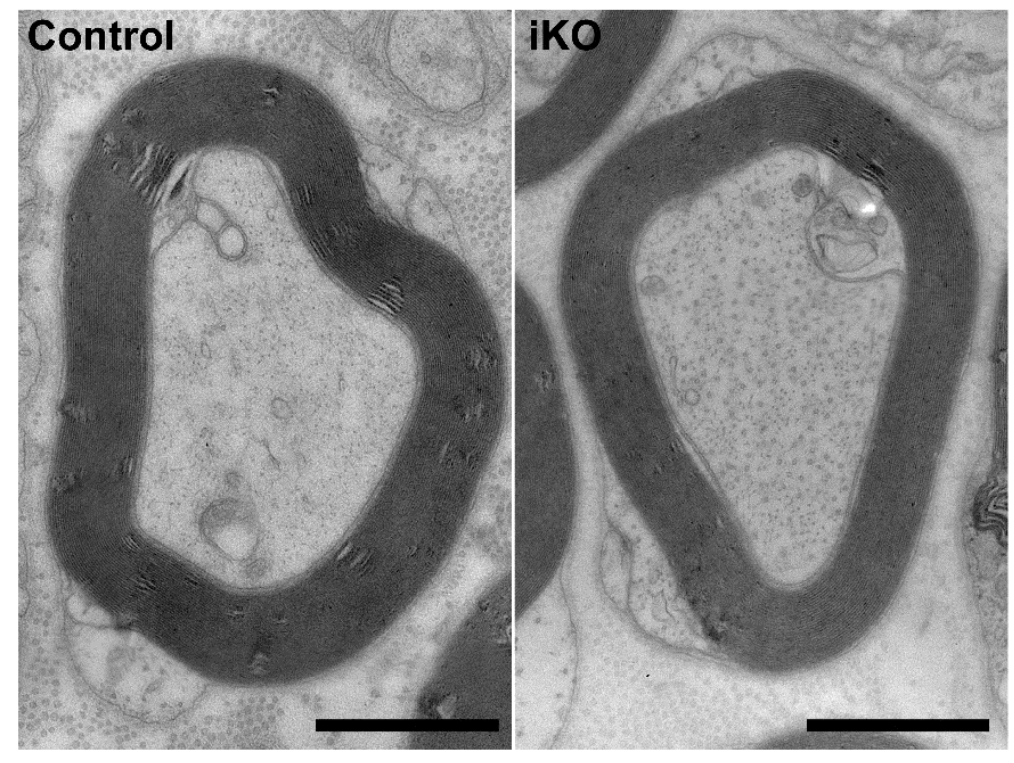

Figure 3-14 No obvious abnormalities in PNS myelin after MBP knock-out

Ablation of MBP at 8 weeks of age does not result in obvious myelin abnormalities at 16W pti. Transmission electron micrograph of sciatic nerve, scale bar $1 \mu \mathrm{m}$. 


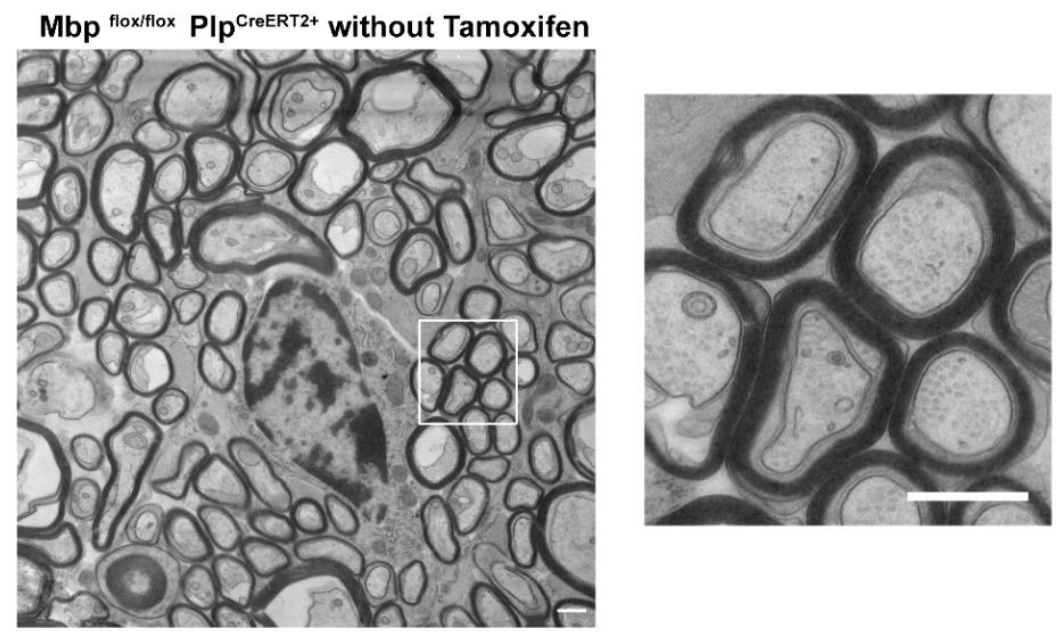

Figure 3-15 No myelin abnormalities in the absence of tamoxifen induced recombination

Myelin appears normal in animals that harbor Mbpflox/flox*PIpCreERT2 but have not received tamoxifen. Electron micrograph of high pressure frozen (HPF) and freeze substituted (AFS) optic nerve at an age of 36W, scale bar $500 \mathrm{~nm}$.

To verify that recombination and resulting structural changes do not appear without

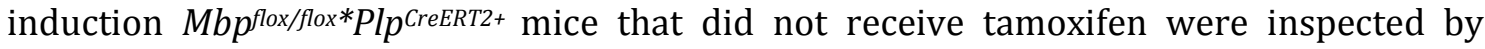
electron microscopy. The optic nerve of mice at $36 \mathrm{~W}$ of age, which is the matching age for mice at $26 \mathrm{~W}$ post tamoxifen induction showed no myelin abnormalities and or increased membrane tubules at the inner tongue (Figure 3-15). From this we concluded that the observed motor phenotype can be attributed most likely to the loss of MBP in the CNS and the resulting demyelination.

\subsection{MBP has little lateral mobility within the myelin sheath}

Next, we addressed the question whether MBP is selectively lost from the myelin sheath or the compact myelin compartment itself is shrinking. Immunoblot analysis at $26 \mathrm{~W}$ pti from brain lysate (Figure 3-16, A) and the isolated myelin from the same lysate (Figure 3-16, D) showed that MBP and PLP abundance was decreased only in the lysate but not in the myelin fraction (Figure 3-16, E). The reduced levels of MBP and PLP in the lysate indicate a reduced myelin yield from iKO brains (Figure 3-16, B, C). From these data we concluded that the abundance of the compact myelin compartment per se is reduced in knock-out animals and that the enriched compact myelin fraction is defined by and dependent on presence of MBP. This finding also implies that MBP has little lateral mobility, since the composition of the remaining myelin is unchanged regarding MBP and PLP abundance. To test this hypothesis at the ultrastructural level, MBP immunogold labeling was performed on optic nerve cryosections from mice at $26 \mathrm{~W}$ pti. The area covered by compact myelin was significantly reduced by $50 \%$ (Figure $3-17, A$ ) which is in accordance with the previously described decrease in myelinated profiles in the optic nerve (Figure 3-10, B). The density of MBP labeling gold particles was unchanged on compact myelin in knock-out animals in comparison to controls. This is indicating that the abundance of MBP within the compact myelin was indeed unchanged as expected (Figure 3-17, B). From these experiments we conclude that MBP has limited lateral mobility and is not diluted out of the compact myelin after Mbp ablation. 


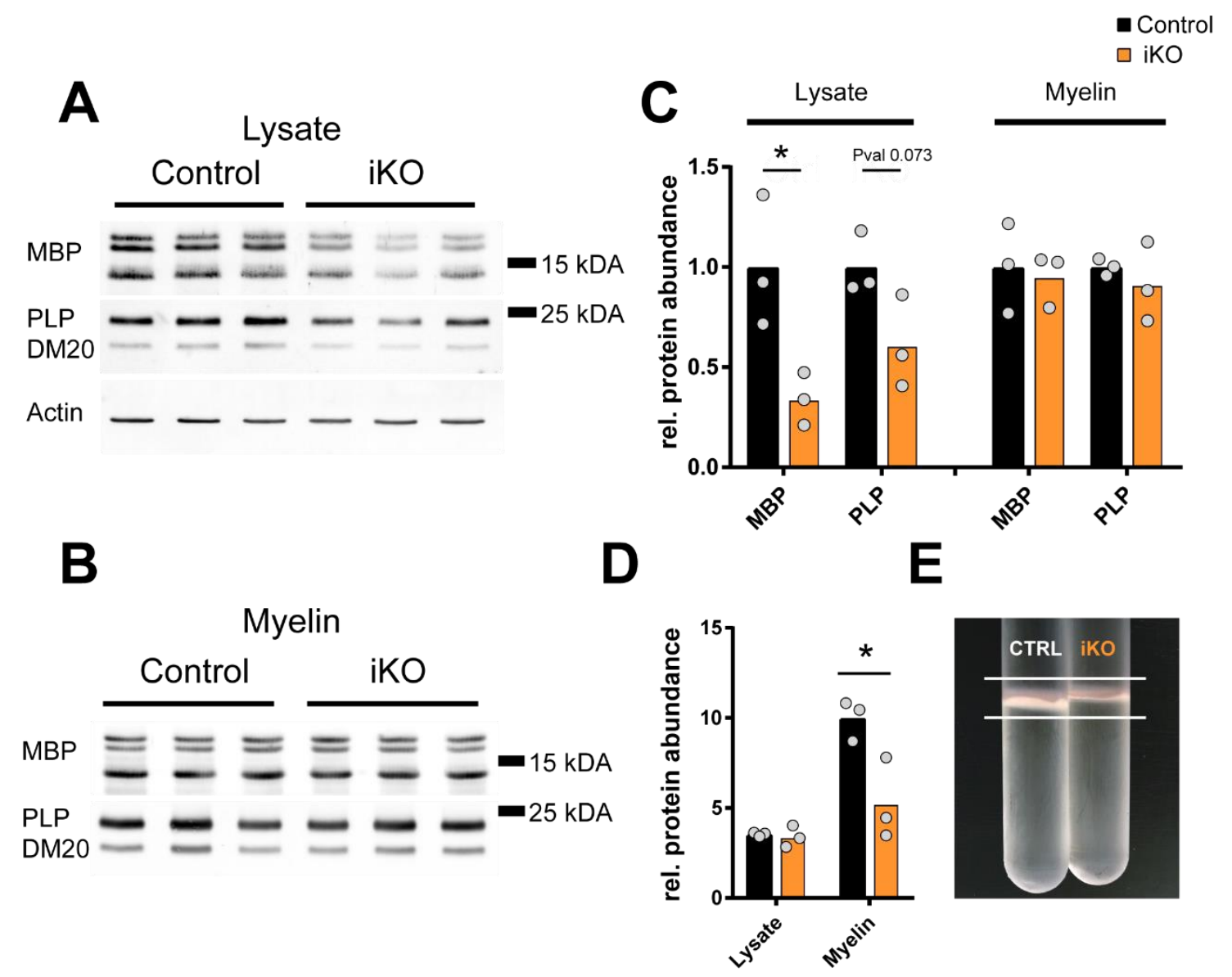

Figure 3-16 MBP and PLP abundance in isolated myelin is not changed

MBP and PLP levels are diminished in immuno-blot of total brain lysate from mice 26W pti (A) changes in abundance are not found in the isolated myelin fraction $(B, C)$. The amount of compact myelin is decreased by approximately $50 \%$ in iKO compared to control mice as measured by Bradford protein assay (Bio-Rad) (D) and myelin mass is reduced in the second sucrose gradient step in the myelin isolation protocol (E). Brain lysate from one hemisphere was used for myelin isolation, two-tailed unpaired $t$-test; $p<.05\left(^{*}\right)$. 

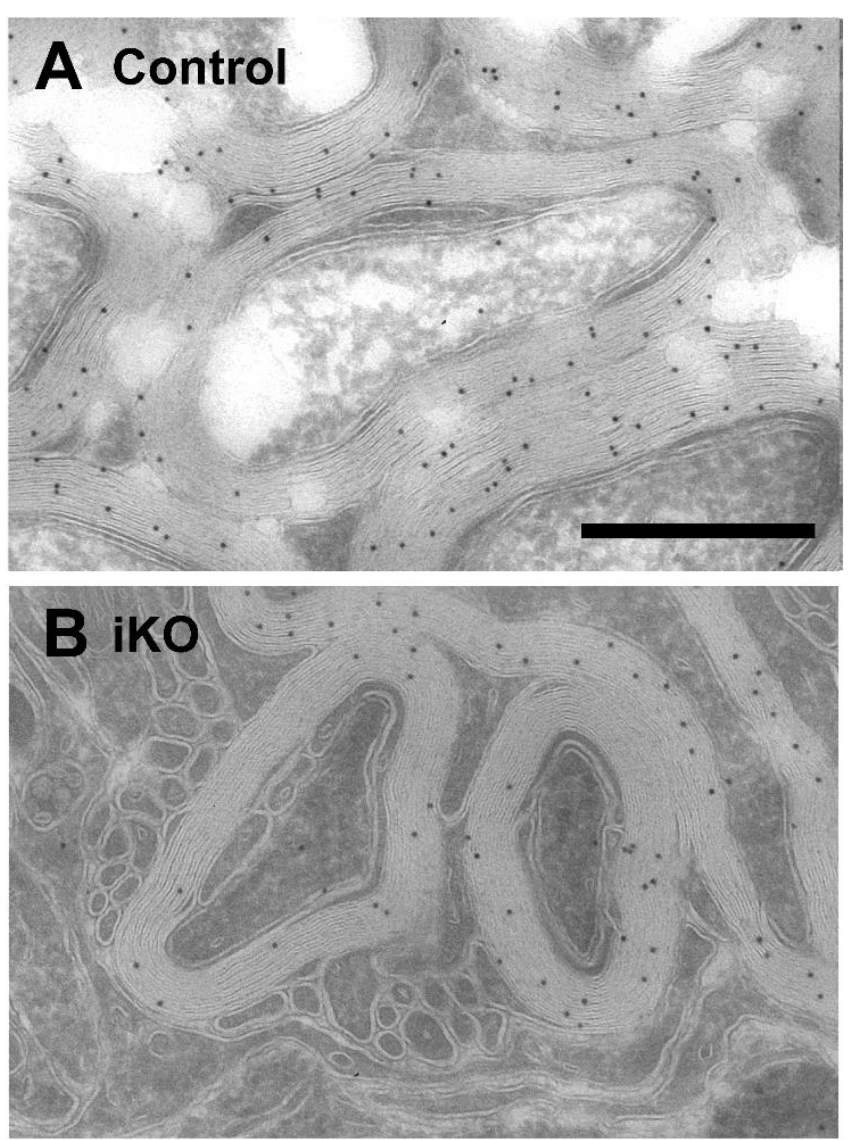

C
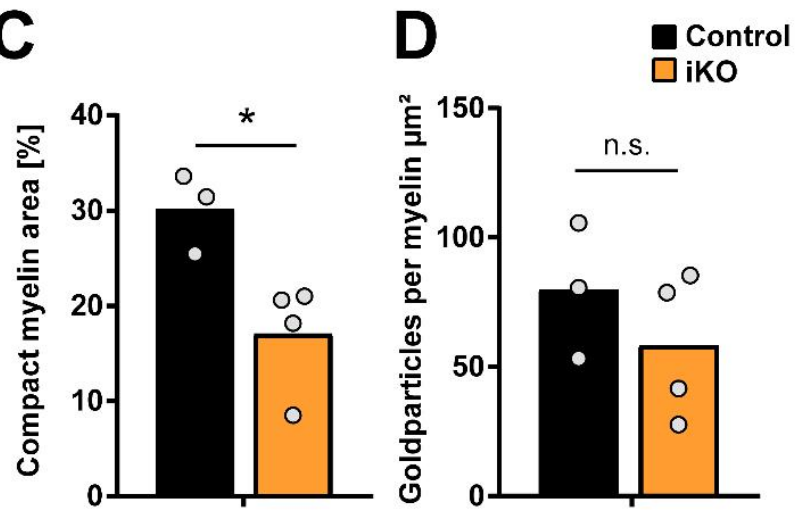

$26 \mathrm{~W}$ pti.

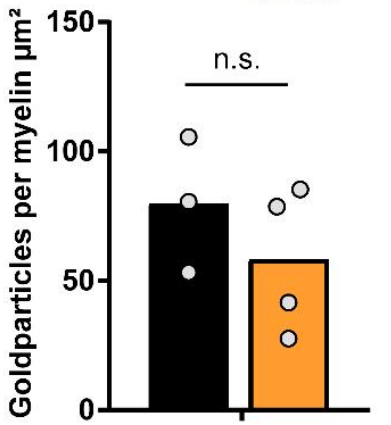

26W pti.

Figure 3-17 MBP has limited lateral mobility within the myelin sheath

The labeling density of MBP in control myelin (A) is comparable to iKO myelin (B) suggesting little reduction in MBP content within the compact myelin of iKOs (D). The section area occupied by myelin is significantly decreased after MBP iKO (C). Labeling of MBP with a custom-made antibody (Kathrin Kusch), 10nm protein-Agold particles. At least 4 sections with in total $300 \mu \mathrm{m}^{2}$ per animal were quantified using a $2 \mu \mathrm{m}$ grid to randomly select axons, $\mathrm{N}=3-4$ animals, two-tailed unpaired $t$-test; $p<.05\left(^{*}\right)$ scale bar $500 \mathrm{~nm}$. 


\subsection{Proteom analysis of the optic nerve after MBP iKO}

To study changes in myelin composition and systemic responses after ablation of MBP, mass-spectrometry measurements and statistical evaluation were performed by the team of Dr. Olaf Jahn (Proteom Facility, Max-Planck-Institute for Experimental Medicine). Since the myelin fraction would mostly enrich compact parts of the myelin but, judging from the discussed unchanged MBP content in the enriched myelin fraction, probably not the observed non-compact membrane tubules (Figure 3-16) the optic nerve lysate was chosen as the model of choice. The optic nerve can be isolated easily and reproducibly in toto and represents a model-white matter tract with a limited amount of cell types. Whole optic nerve lysates from MBP iKO and control animals $8 \mathrm{~W}, 16 \mathrm{~W}$ and $40 \mathrm{~W}$ pti as well as shiverer and control mice at an age of 10 weeks were analyzed. We included shiverer optic nerves into the analysis to be able to assess similarities and differences between the demyelinating model of the induced MBP ablation and the model that never possessed MBP.

A

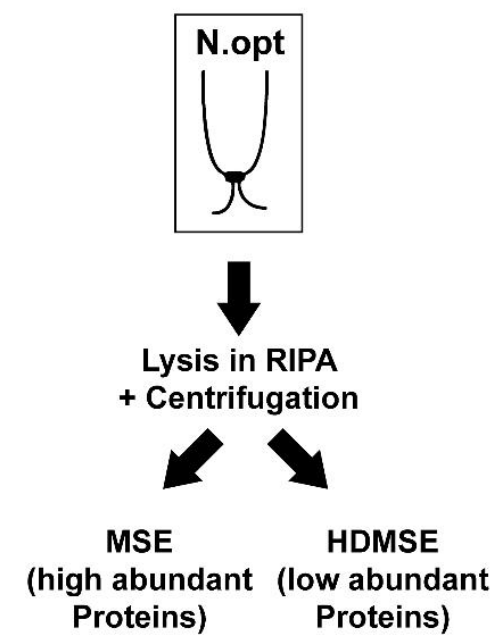

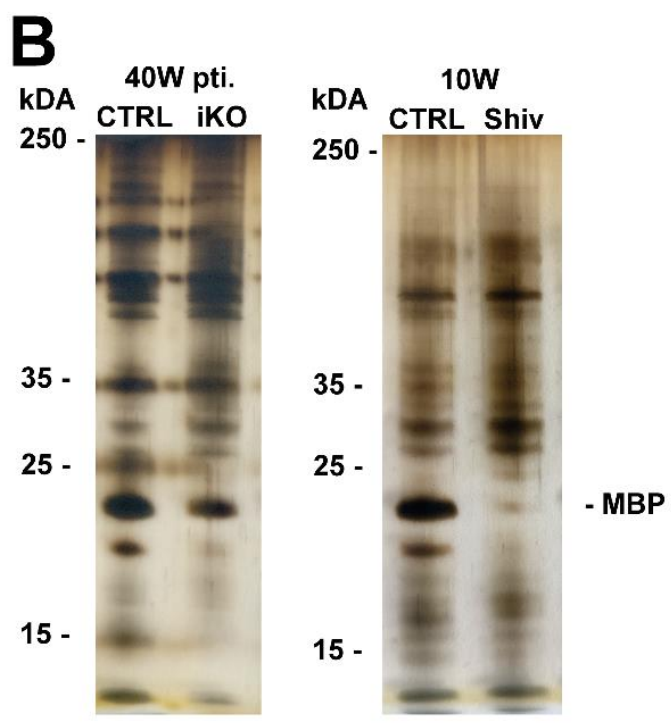

Figure 3-18 Proteom analysis of optic nerve lysate

Optic nerve lysate from male MBP iKO at and 10W old shiverer were subjected to mass spectrometry $(\mathrm{N}=4)$. High abundant proteins such as MBP and PLP were quantified using the MSE approach, for less abundant proteins the more sensitive HDMSE method was used. Mass-spectrometric measurements were performed by the team of Dr. Olaf Jahn at the Max-Planck-Institute of Experimental Medicine as previously described (A). Silver staining of proteins in polyacrylamide gels of optic nerve lysate $40 \mathrm{~W}$ pti and $10 \mathrm{~W}$ old shiverer was performed as quality control and reveals decreased levels of MBP (B). 
To quantify the top abundant myelin proteins such as MBP, PLP or CNP measurements were performed in the MSE mode, which allows precise quantification of highly abundant proteins. For quantification of lower abundant proteins samples were measured in high definition mass spectrometry (HDMSE) acquisition mode which enables the detection of low abundant peptides but yields less precise abundance measurements for higher abundant peptides (Figure 3-18, A). To verify sample quality silver staining of proteins in polyacrylamide gels were performed for every sample exemplary shown for $40 \mathrm{~W}$ pti and shiverer. (Figure 3-18, B). Vulcano plots of $\log _{2}$ fold changes and q-values showed many proteins significantly changed in abundance and revealed an expected distribution validating the quality of the data (Figure 3-19). The relative protein abundance as measured by MS ${ }^{\mathrm{E}}$ showed a progressive shift in lysate composition. Major myelin proteins like MBP, PLP, and CNP were progressively less represented in the complete protein amount reflecting a reduction of the compact myelin compartment (Figure 3-20).
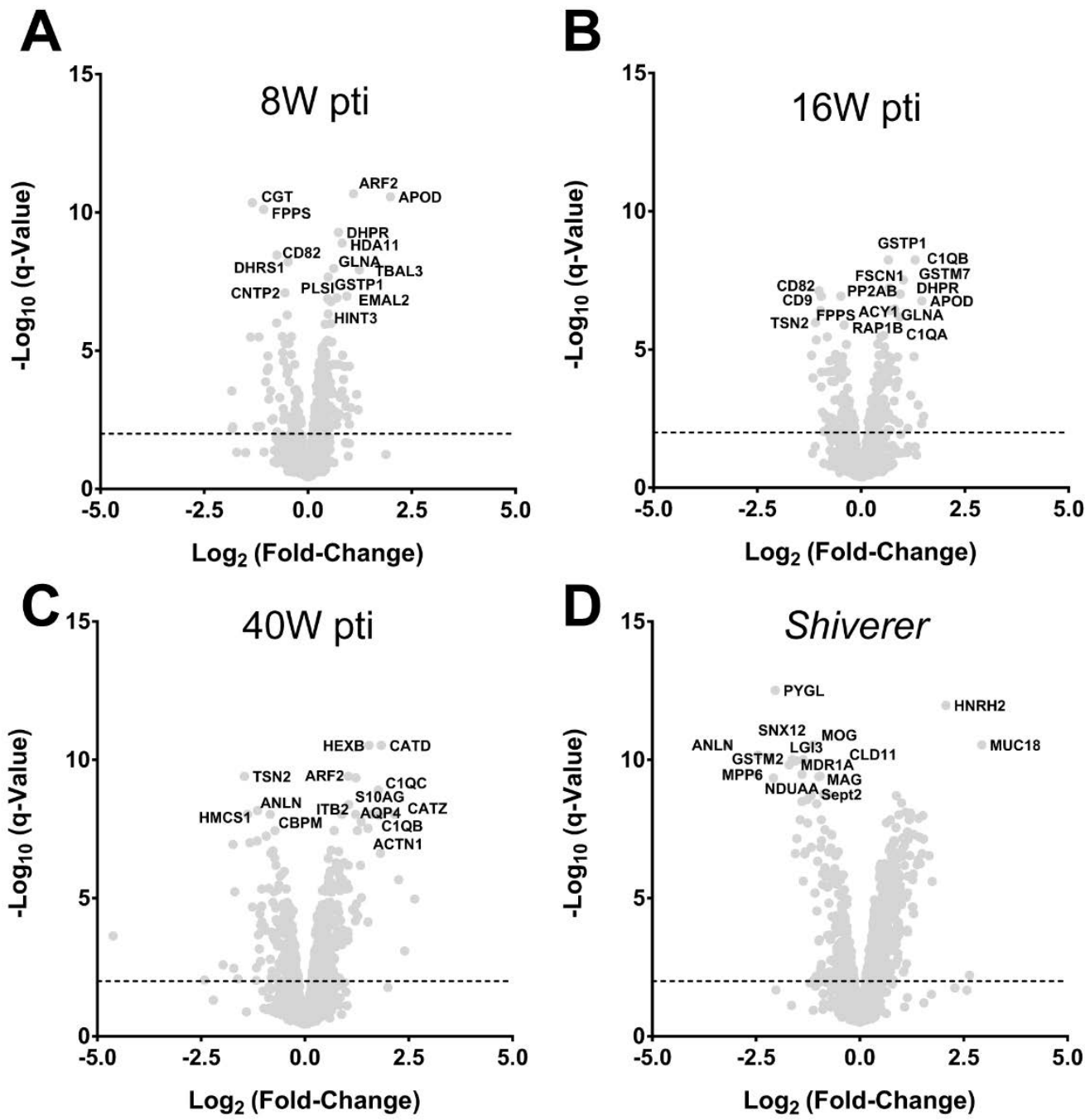

Figure 3-19 Vulcano plots of optic nerve proteome

Vulcano plots of HDMSE measured with Top 15 most significantly regulated genes labeled. Number of detected proteins 8W (1500), 16W (1447), 40W (1644) and shiverer (1677). Dashed line: q-Value = 0.05. q-Value was calculated using the LIMMA package and performed by Lars Piepkorn (Proteom facility, Max-Planck institute for Experimental Medicine) 


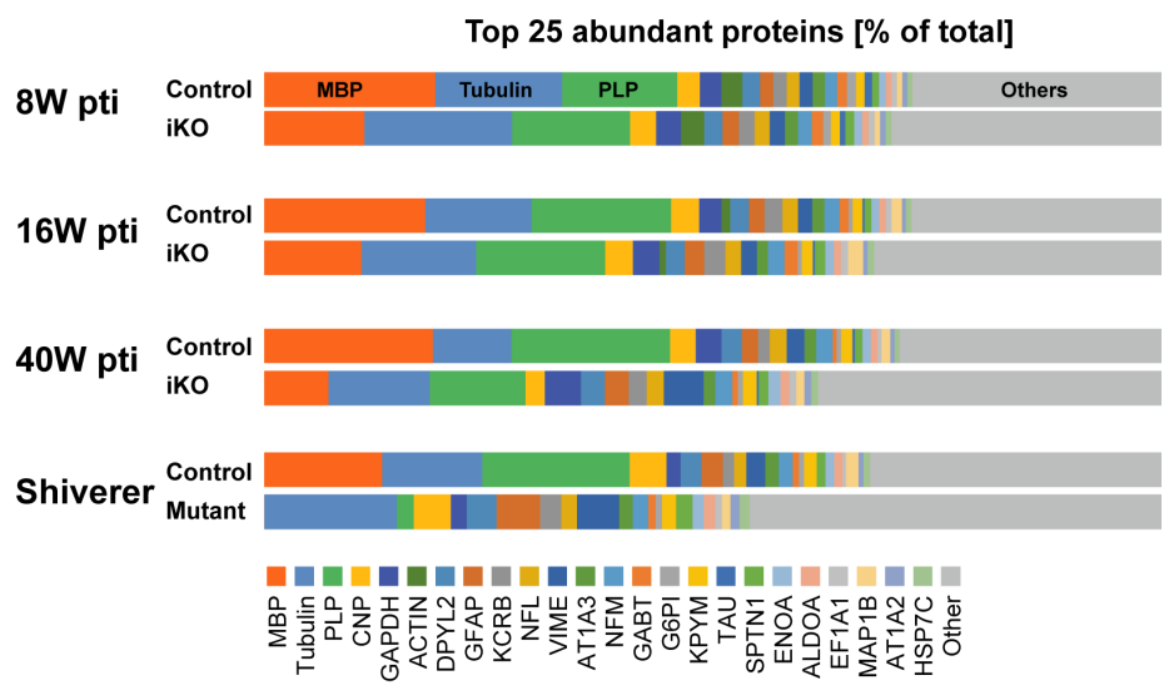

Figure 3-20 Relative protein composition shows reduction in major myelin proteins

Top 25 most abundant proteins as found in control at 8W pti. Data obtained by MSE and plotted as percent of total protein amount as quantified by Isoquant. Tubulin isoforms could not reliably be distinguished and were pooled. Spectrins were excluded due to unreliable quantification for technical reasons.

The loss of MBP by ablation in the adult myelinated animal in Mbpflox/flox*PlpCreERT2 + or the complete absence of MBP in the shiverer mice results in major changes in protein composition. A q-value threshold of $<0.05$ was set to determine significant changes resulting in an increase of abundance for 176 proteins and a decrease for 565 proteins in nerve lysate $8 \mathrm{~W}$ pti. At $16 \mathrm{~W}$ pti 250 proteins were found to be increased and 234 were decreased followed by 878 increased and 351 decreased proteins in optic nerve lysate $40 \mathrm{~W}$ pti. The highest number of changed proteins was found in shiverer optic nerve lysate with 389 proteins decreased and 1034 significantly increased in abundance compared to the control.

The proteins most significantly decreased in abundance for $8 \mathrm{~W}$ and $16 \mathrm{~W}$ pti (Figure 3-21, A ,B) include for example myelin proteins such as MBP, CD9 and Opalin, proteins associated to lipid synthesis such as farnesyl diphosphate synthase (FPPS) or UDP glycosyltransferase 8 (CGT) and adhesion proteins like protein 4.1, CD81 or neural cell adhesion molecule 1 (NCAM1). Increased in abundance are proteins associated with lipid transport such as APOD and APOE, proteins of the cytoskeleton like ADP-ribosylation factor 2 and 4 (ARF2/4), fascin (FSCN1) or tubulin (TBAL3) and proteins associated with stress response such as glutathione S-transferase P 1 (GSTP1) or alpha-crystallin B chain (CRYAB).

For $40 \mathrm{~W}$ pti the top proteins significantly reduced in abundance include myelin proteins like MBP, PLP (MPYR), MOG, MAG, CN37 (CNP) and Opalin and cell adhesion molecules like tetraspanin 2 (TSN2) and CD82. The proteins most increased in abundance are microglial and degradative proteins such as hexokinase B (HEXB), cathepsin D/Z/S (CATD, CATZ,CATS), components of the complement system (C1QC; C1QB) and components of the cytoskeleton such as vimentin (VIM) alpha actinin-1 (ACTN1) and actin-related protein $2 / 3$ complex subunit 1B (ARC1B) (Figure 3-21, C).

In shiverer the top 25 proteins significantly reduced in abundance are mostly myelin proteins such as MOG, PLP (MYPR), claudin11 (CLD11), MAG and cytoskeletal proteins like anilin (ANLN), septin 2 and 4 (SEPT2/4). Increased in abundance are ribosomal proteins such as 60S ribosomal protein L4/L7a/L5 (RPL4/7a/5) lipid associated proteins such as 
APOE, long-chain-fatty-acid--CoA ligase (ACSBG1), fatty acid-binding protein (FABP7) and energy metabolism related proteins such as phosphoglycerate mutase 2 (PGAM2), creatine kinase B-type (KCRB) and ATPase subunit alpha-1 (AT1A1) (Figure 3-21, D)

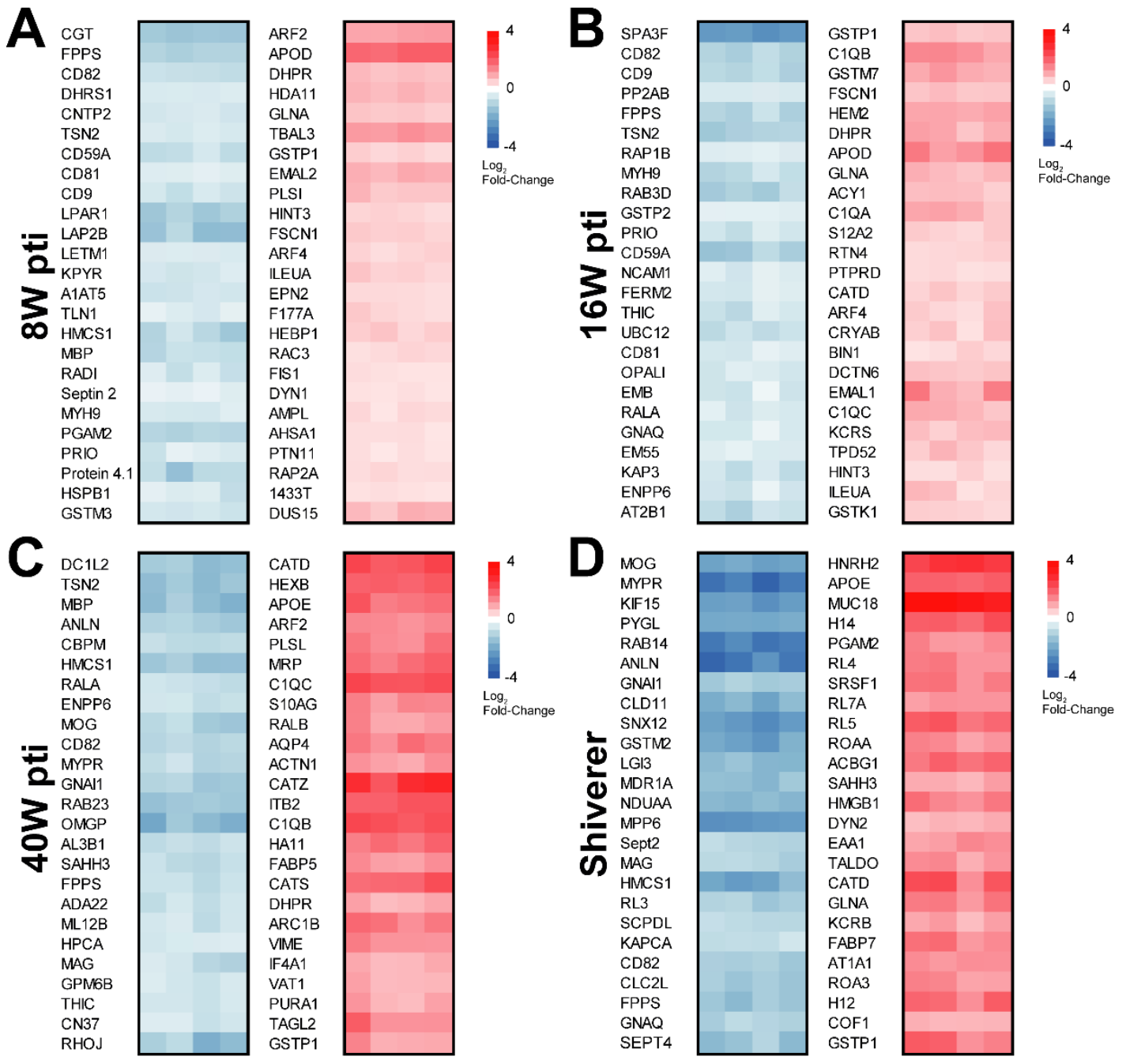

Figure 3-21 Top 25 significantly increased and decreased proteins in optic nerve

(A-D) Top 25 significantly increased and decreased proteins in optic nerve lysate from iKO and control mice 8W, $16 \mathrm{~W}$ and $40 \mathrm{~W}$ pti and from shiverer and control at the age of 10W. Proteins with reduced abundance include for example myelin proteins (MBP, MPYR (PLP), MAG, MOG), proteins of the cytoskeleton (ANLN, SEPT), cell adhesion molecules (tetraspanin 2, (TSPAN2), CD9, NCAM1) and lipid synthesis (farnesyl diphosphate synthase (FPPS), UDP glycosyltransferase 8 (CGT). Proteins with increased abundance include for example lipid transport proteins (APOD, APOE), degradative enzymes (cathepsin D (CATD) and Z (CATZ)), cytoskeleton associated proteins (ARF2, ARF4) and marker for microglia (hexokinase B (HEXB)) and astrocytes (aquaporin 4(AQP4)). Mean of Log2 Fold change of 4 technical replicates per animal, plotted on a color scale with red represening enrichment and blue deminishment in iKO compared to control. Protein identifies represent Uniprot protein IDs of mus musculus. 
Pathway enrichment analysis was performed on proteins significantly increased in abundance with a q-Value threshold of less than 0.05 . Analysis of the early time point $8 \mathrm{~W}$ pti, the late time point 40W pti and shiverer animals was performed and mapped on KEGG pathways using the freely available web tool g-profiler (https://biit.cs.ut.ee/gprofiler). KEGG pathway analysis revealed an enrichment of proteins assigned to mitochondrial processes such as oxidative phosphorylation or the TCA cycle and for proteins associated with ribosomes (Figure 3-22, A-C). In samples of 40W pti and shiverer an enrichment was found for proteins associated with glycolysis and degradative processes such as the proteasome and the phagosome (Figure 3-21 B,C).

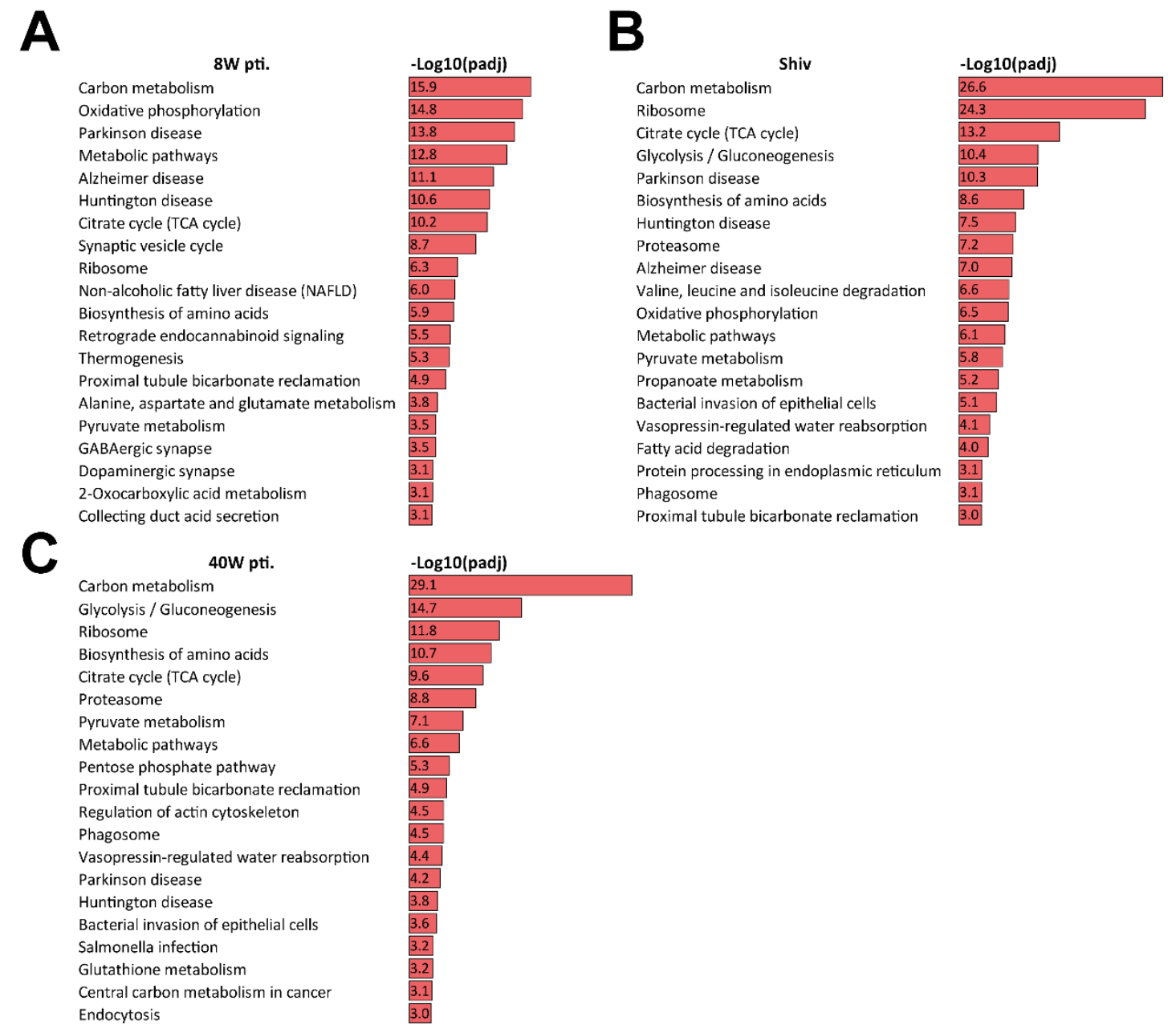

Figure 3-22 Pathways enrichment of proteins with significantly increased abundance

(A-C) Top 20 enriched KEGG pathways in optic nerve lysate $8 \mathrm{~W}$ pti, 40W pti and 10W old shiverer reveals enrichment in mitochondrial processes (Oxidative phosphorylation, TCA cycle) and proteins associated to the ribosome (A, B, C). For 40W pti and shiverer enrichment of glycolysis related proteins and proteasome pathway are found (B, C). Enrichment was statistically corrected by the g:SCS threshold as given by g-profiler. Enrichment analysis was performed against the mus musculus background provided in g-profiler.

In summary the proteome analysis reveals a large number of proteins significantly changed in abundance of which the most interesting ones belonging to the group of stress related proteins, the increase in mitochondrial proteins and cell adhesion as well as cytoskeletal proteins across all 4 conditions. The datasets are available as a resource for future analysis. 


\subsection{Lipid analysis of optic nerve after MBP iKO}

Changes in lipid composition were reported in white matter of an experimental autoimmune encephalomyelitis model of marmosets (Ohler et al., 2004). White matter lesions contained increased levels of phosphatidylserine and unsaturated fatty acids including cholesterol esters. The levels of sphingomyelin, phosphatidylcholine, and cerebroside sulfates were decreased. These changes were hypothesized to increase membrane fluidity and curvature resulting in reduced adhesion of MBP resulting in formation of membrane vesiculations (Ohler et al., 2004). Based on the previously published results we investigated the lipid composition after knock-out of $M b p$.

Lipid analysis by mass spectroscopy of optic nerves and corpus callosum $8 \mathrm{~W}$ and 16W pti was performed in collaboration with Prof. Dr. Britta Brügger (Biozentrum Heidelberg) to study the consequences of $M b p$ ablation on the lipid composition (Figure 3-23). A minor reduction of cholesterol (Chol) levels was observed in optic nerve 8W pti (Figure 3-21, A) and a comparable non-significant trend in corpus callosum 8W pti (Figure 3-21, C). Levels of lyso-phosphatidyl choline (LPC) were increased in corpus callosum and optic nerve 8W pti and showed a non-significant trend towards increase 16W pti (Figure 3-21. A-D). Levels of phosphatidylglycerol (PG) were increased in corpus callosum $8 \mathrm{~W}$ and $16 \mathrm{~W}$ pti and showed a strong but due to high variation non-significant increase in optic nerve $16 \mathrm{~W}$ pti. For phosphatidylserine (PS) a minor increase is observed in corpus callosum $8 \mathrm{~W}$ and $16 \mathrm{~W}$ pti. Furthermore, a strongly significant increase of phosphatidylinositol (PI) levels was observed for optic nerve $16 \mathrm{~W}$ as well as a non-significant increase in corpus callosum $16 \mathrm{~W}$ pti. The abundance of cholesteryl ester (CE) showed a trend towards decrease in corpus callosum $8 \mathrm{~W}$ pti and in optic nerve $8 \mathrm{~W}$ and $16 \mathrm{~W}$ pti. Since the lipid analysis was performed on heterogeneous tissue samples, we are not sure about the interpretation of these results. It is possible that too many cell types with their cell-type specific responses were contained in the sampled area. On the other hand, we did not expect to find differences in the myelin fraction and therefore have chosen this experimental approach. 
A

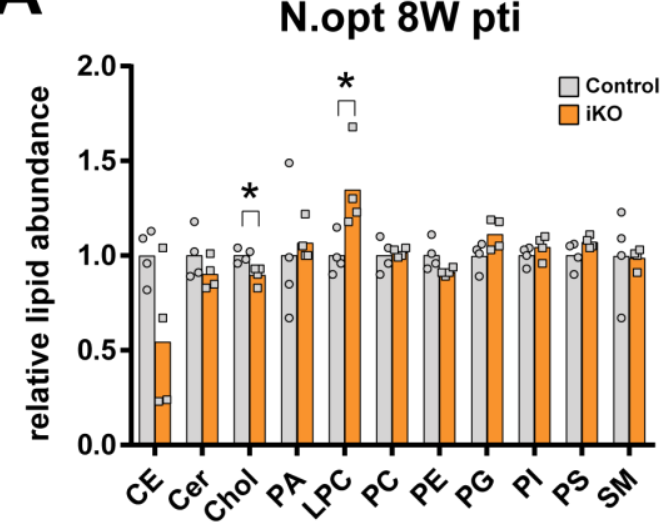

C

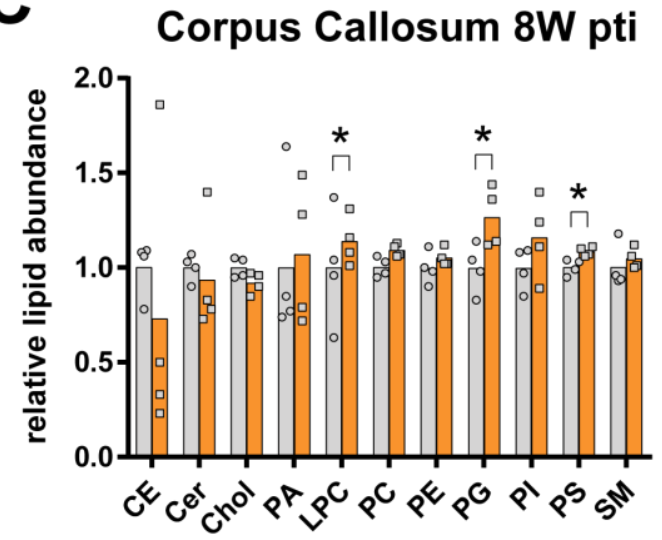

B

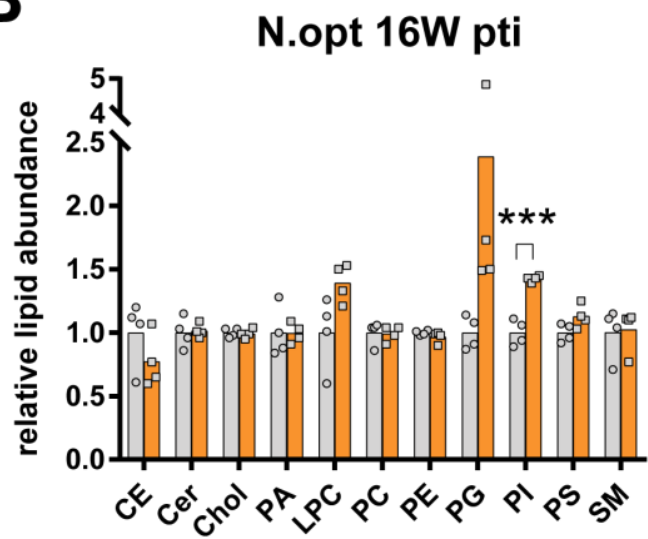

D
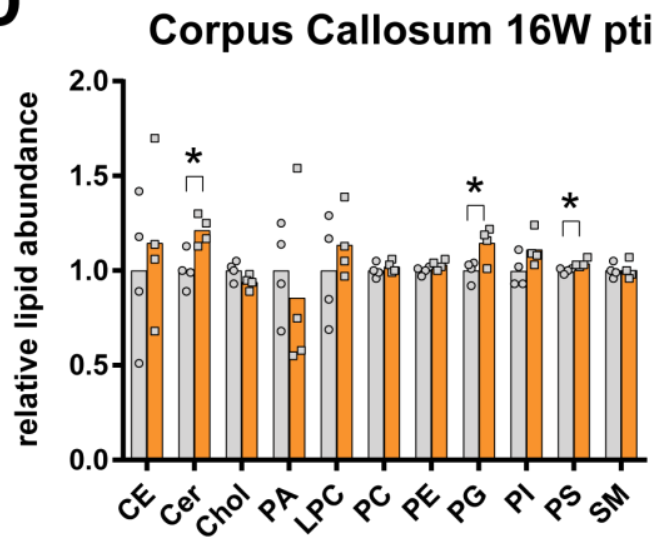

Figure 3-23 Lipidome analysis after MBP iKO

Lipid composition analysis by mass spectrometry of optic nerve 8W pti (A), 16W pti (B) and corpus callosum $8 \mathrm{~W}$ pti (C) and 16W pti (D) reveal minor changes in lipid composition after MBP knock-out. Lipids: Cholesteryl ester (CE), Ceramide (Cer), Cholesterol (Chol), Lyso-Phosphatidyl Choline (LPC), Phophatidic acid (PA), Phosphatidylcholine (PC), Phosphatidylethanolamine (PE), Phosphatidylglycerol (PG), Phospatidylinositol (PI), Phospatidylserine(PS), Sphingomyelin (SM). N=4 samples obtained from individual animals. two-tailed unpaired $t$-test, Lipid analysis was performed by the team of Prof. Dr. Britta Brügger (Biozentrum Heidelberg).

\subsection{Microglial activation and astrogliosis upon MBP iKO}

Since demyelination is known to trigger inflammation (Peterson and Fujinami, 2007), analysis of the neuropathological markers GFAP, MAC3, APP and CD3 was performed. Immunohistochemistry on fimbria was carried out for the time points $8 \mathrm{~W}, 16 \mathrm{~W}, 26 \mathrm{~W}$ and $52 \mathrm{~W}$ pti and for the corticospinal tract (CST) of the spinal cord at the time points $8 \mathrm{~W}, 16 \mathrm{~W}$ and 26W pti. An increase in GFAP+ area indicating an astrogliosis was first observed $16 \mathrm{~W}$ pti together with an increase in CD3 positive immune cells and a minor increase in MAC3+ area indicating the onset of a microgliosis (Figure 3-24, A- C). At 26W pti the MAC3 ${ }^{+}$area is increased and APP positive spheroids are present (Figure 3-24, A- D). Astrogliosis and the number of $\mathrm{CD}^{+}$cells persist and the number of APP spheroids and $\mathrm{MAC}^{+}$area is further increased at the late time point at $52 \mathrm{~W}$ pti. 

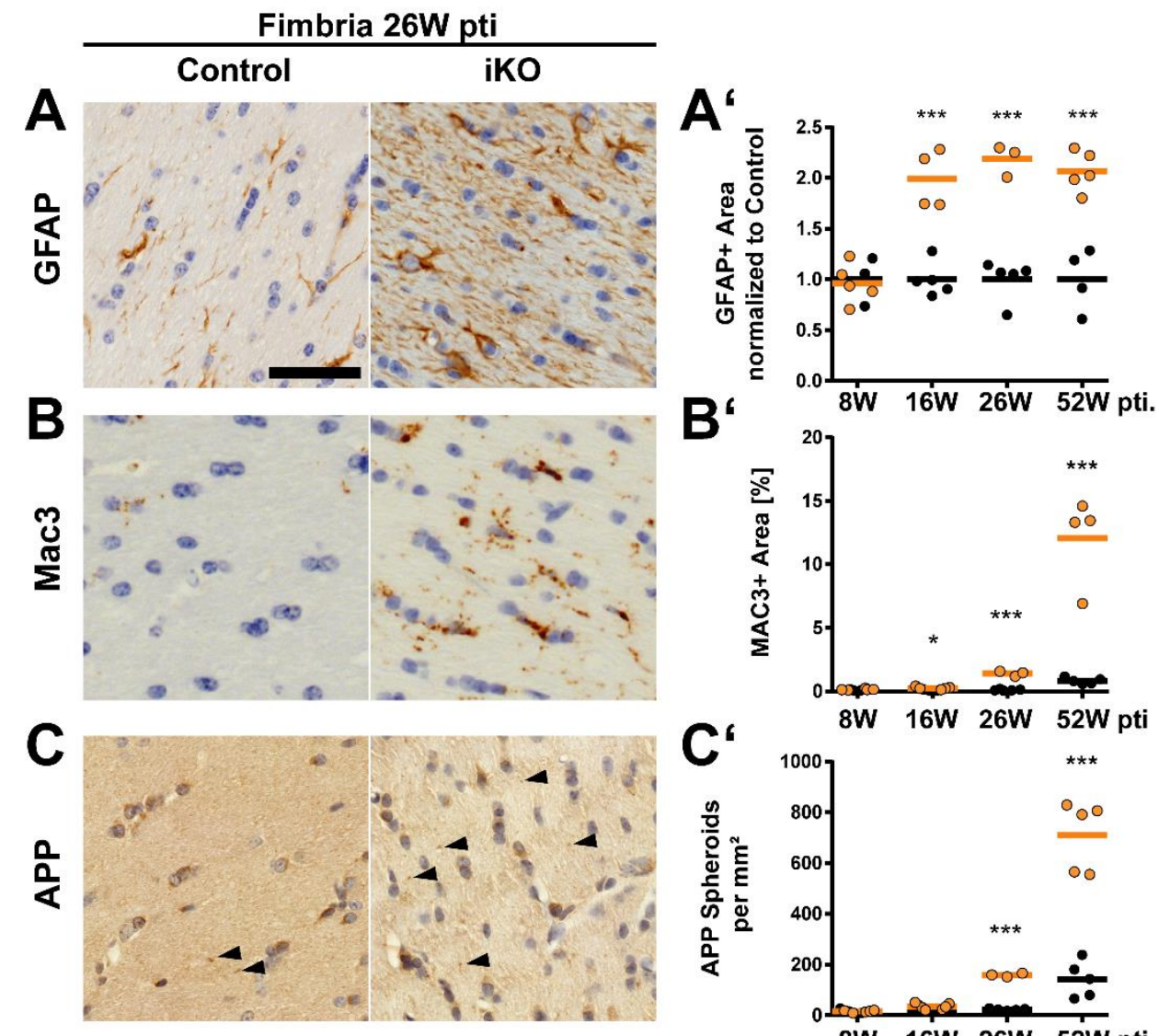

B
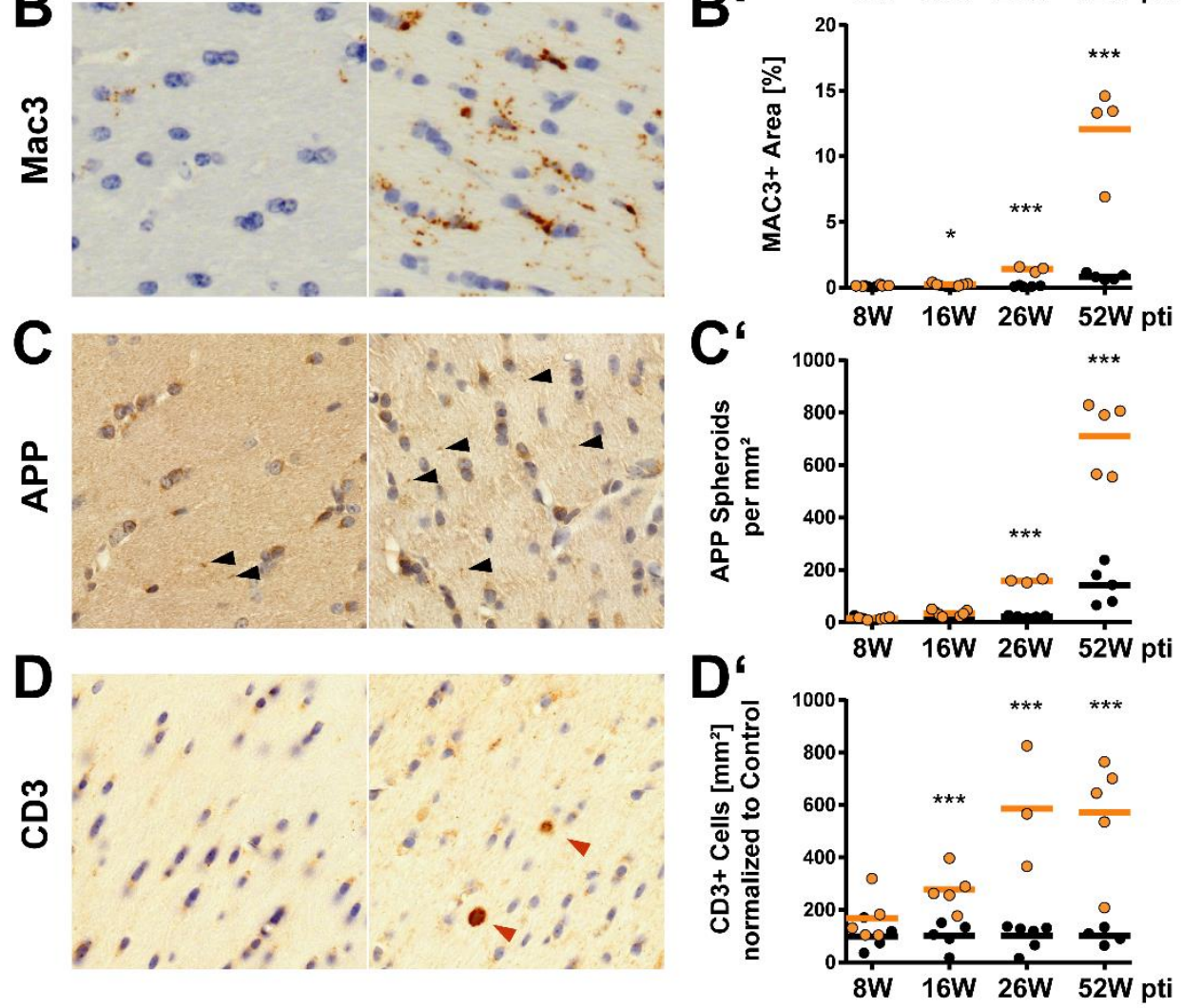

Figure 3-24 Progressive low grade astrogliosis, microgliosis and axonal pathology in Fimbria

An increase in GFAP+ area (A) indicates astrogliosis beginning between 8 and 16W pti ( $\left.\mathrm{A}^{\prime}\right)$. The increase of $\mathrm{MAC}^{+}$area (B) indicates increasing microglial activity beginning between 8 and $16 \mathrm{~W}$ pti and increasing until $52 \mathrm{~W}$ pti (B'). Axonal pathology observed by labeling of APP spheroids (C) appears late at 26W pti and increases until 52W pti ( $\left.C^{\prime}\right)$. Increased numbers of T-cells (D) can be observed already at $16 \mathrm{~W}$ pti. showing immune system activation (D'). N=3-5 animals, 2 fimbria (GFAP, MAC3, APP) or 6 (CD3) fimbria per animal, two-tailed unpaired $t$-test; $p<.05\left(^{*}\right), p<.01\left(^{* *}\right), p<.001\left(^{* * *}\right)$, scale bar $50 \mu \mathrm{m}$. 
Neuropathological analysis of the spinal cord corticospinal tract (CST) at 8W, 16W and 26W pti was performed by immunohistochemistry of GFAP, MAC3 and APP (Figure 3-25, A-C). In spinal cord a significant increase in $\mathrm{GFAP}^{+}$area was first observed $26 \mathrm{~W}$ pti while a beginning microgliosis is already detected at $16 \mathrm{~W}$ pti (Figure 3-25, A, B). In contrast to the fimbria a significant increase in APP spheroids was observed as early as $8 \mathrm{~W}$ pti and further increased at $16 \mathrm{~W}$ and $26 \mathrm{~W}$ pti. The rather late onset of astrogliosis and microgliosis with limited severity indicates that this mouse model is a suitable tool to study myelin maintenance in a relatively unperturbed physiological system with only minor inflammation.

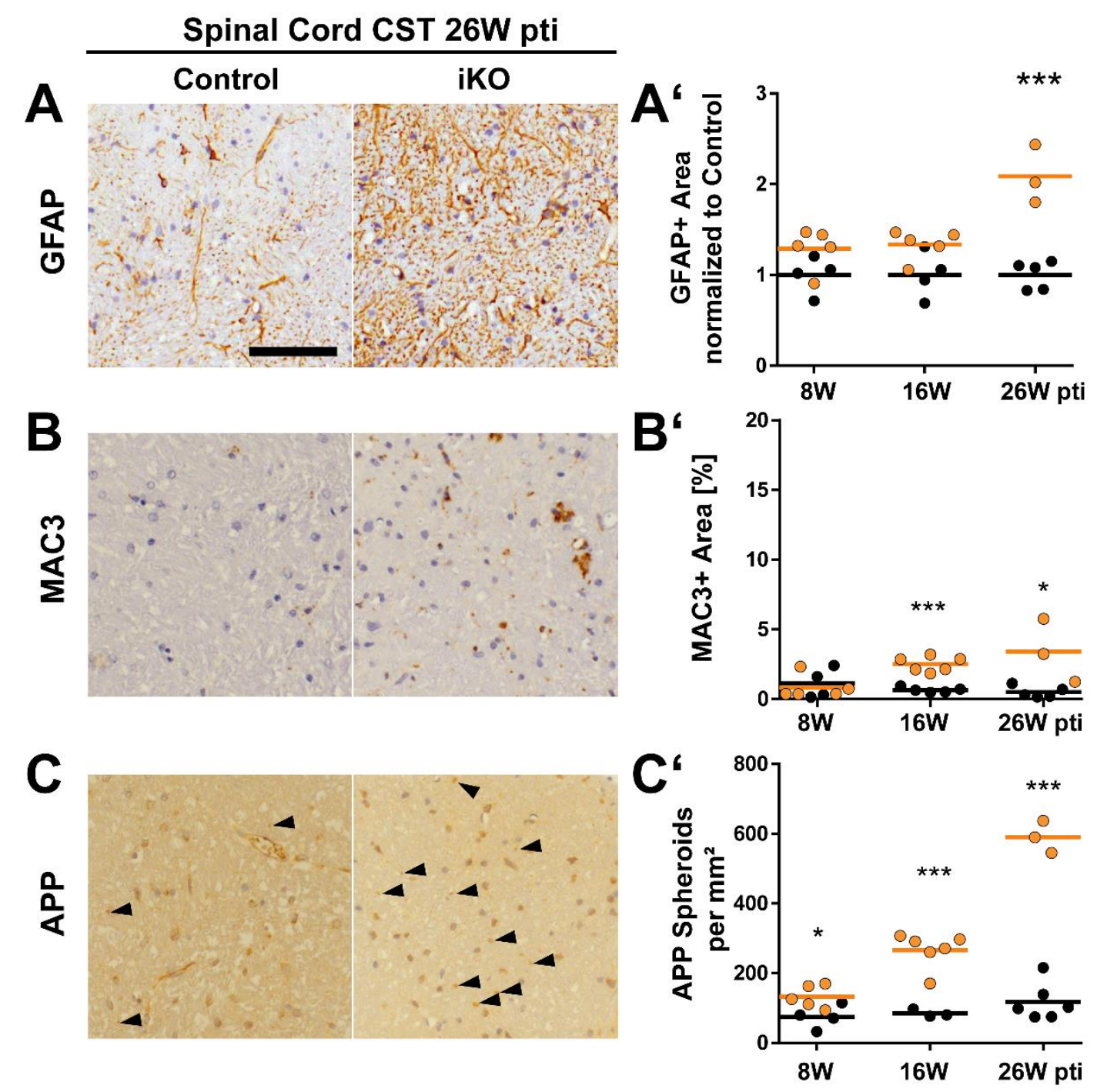

Figure 3-25 Progressive low grade astrogliosis, microgliosis and axonal pathology in the spinal cord An increase in $\mathrm{GFAP}^{+}(\mathrm{A})$ and $\mathrm{MAC3}^{+}(\mathrm{B})$ area in the corticospinal tract (CST) of spinal cord at 26W pti shows astrogliosis $\left(\mathrm{A}^{\prime}\right)$ and microgliosis ( $\left.\mathrm{B}^{\prime}\right)$ Axonal pathology as observed by labeling of APP spheroids ( $\left.\mathrm{C}^{\prime}\right)$ appears already at $16 \mathrm{~W}$ pti and is further increased at $26 \mathrm{~W}$ pti $\left(\mathrm{C}^{\prime}\right) . \mathrm{N}=3-5$ animals, analysis of CST of at least 2 sections per animal, two-tailed unpaired $t$-test; $p<.05\left(^{*}\right), p<.01\left({ }^{* *}\right), p<.001\left(^{* * *}\right)$, scale bar $50 \mu \mathrm{m}$. 


\subsection{Increased density of oligodendrocyte after MBP iKO}

To exclude that the observed inflammation and loss of myelin is caused by oligodendrocyte death, the number of oligodendrocytes and the occurrence of cell death were analyzed in the fimbria. A terminal deoxynucleotidyl transferase dUTP nick end labeling (TUNEL) assay was performed and no significant changes in the number of apoptotic cells were found in fimbria of iKO compared to control animals 40W and 52W pti (Figure 3-26).

To investigate oligodendrocyte and OPC cell numbers and proliferation in response to myelin loss we treated mice at the peak of clinical symptoms ( $40 \mathrm{~W}$ pti) with $0.2 \mathrm{mg} / \mathrm{ml}$ of the thymidine analogue 5-ethynyl-2'-deoxyuridine (EDU) in drinking water. During cell division EDU is integrated into replicating DNA, effectively tagging dividing cells and allowing the identification of cells that underwent cell division during EDU administration (Cavanagh et al., 2011). Mice were treated for three weeks and sacrificed after an additional three-week chase period to be able to observe the differentiation of EDU labeled cells. The number of oligodendrocytes after MBP iKO in the fimbria was assessed by immunohistochemistry of the oligodendrocyte linage marker Olig 2 (Figure 3-27, A), the mature oligodendrocyte marker Carboanhydrase 2 (CAII) (Figure 3-27, B) and the oligodendrocyte precursor marker PDGFR A (Figure 3-27, C). Double labeling with EDU reveals a four-fold increase in Olig $2^{+} \mathrm{EDU}^{+}$as well as in PDGFR $\mathrm{A}^{+} \mathrm{EDU}^{+}$double positive cells. A similar but non-significant trend was observed for $\mathrm{CAII}^{+} \mathrm{EDU}^{+}$positive cells (Figure 3-27, A'-C'). The density of Olig 2, CAII and PDGFR A positive cells was increased (Figure $3-27$, D) $46 \mathrm{~W}$ pti while the area of the fimbria was not significantly changed (Figure 3-27, E). The increase in CAII+ cells was consistently found in fimbria of animal $26 \mathrm{~W}, 46 \mathrm{~W}$ and $52 \mathrm{~W}$ pti (Figure 3-27, F).
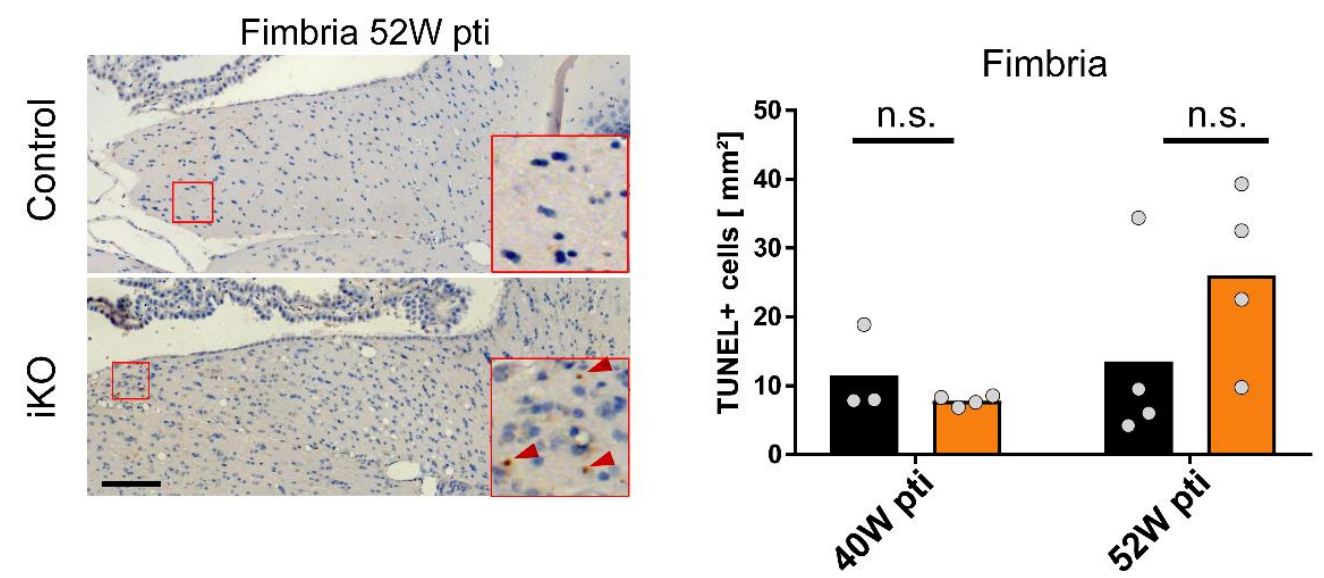

Figure 3-26 No significant increase in cell death after MBP iKO

A TUNEL cell death assay was performed to test for increased cell death in the fimbria of iKO animals showed no significant increase in tunnel positive cells $40 \mathrm{~W}$ and $52 \mathrm{~W}$ pti. Chromogenic labeling, $\mathrm{N}=3-4$ animals, 2 fimbria per animal, two-tailed unpaired $t$-test, scale bar $100 \mu \mathrm{m}$. 


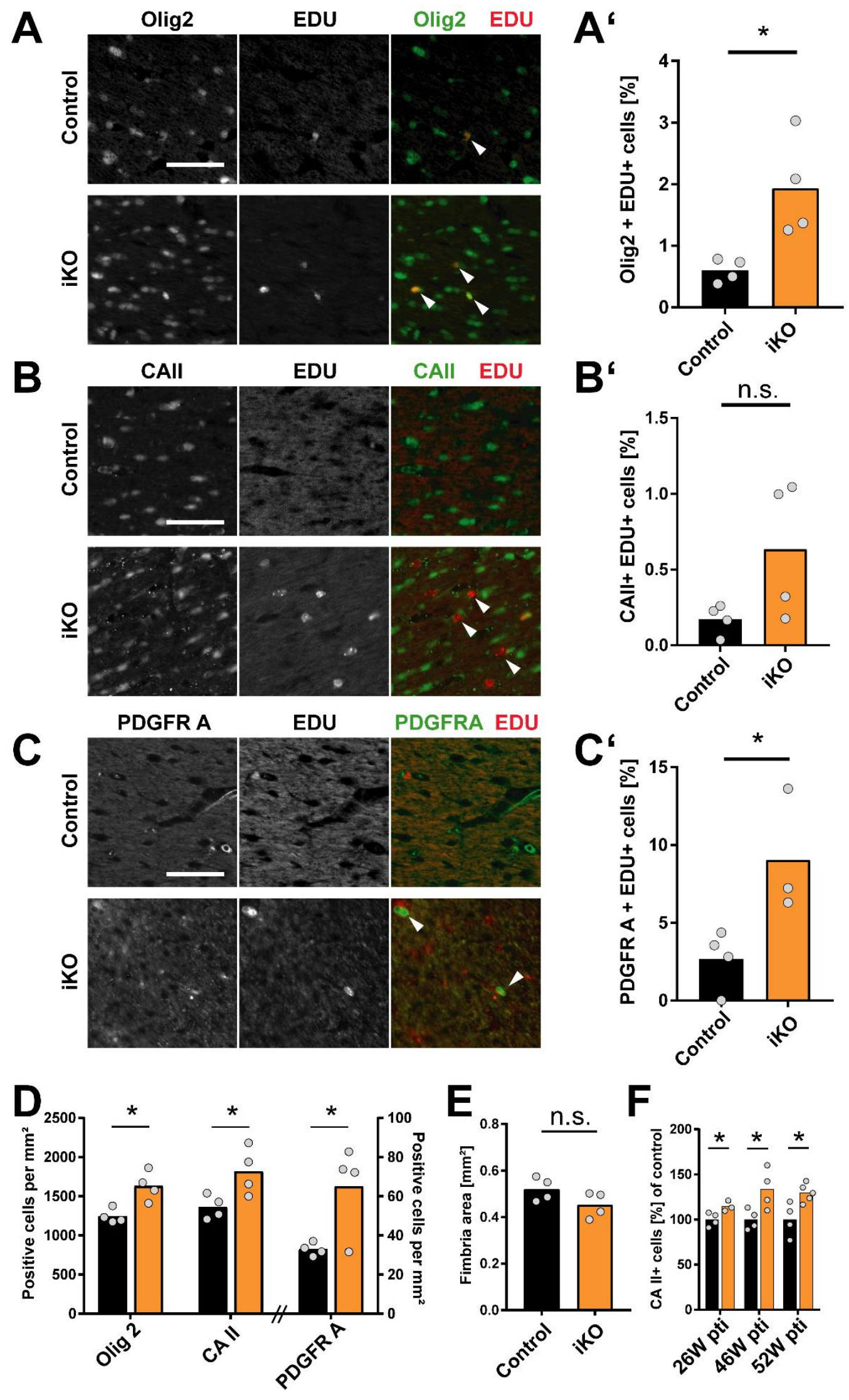


Figure 3-27 Oligodendrocyte numbers are increased after MBP ablation

A-C) To study proliferation of the oligodendrocyte linage cells, mice at $40 \mathrm{~W}$ pti were treated with $0.2 \mathrm{mg} / \mathrm{ml} \mathrm{EdU}$ in drinking water for three weeks followed by a three weeks chase period to allow cell maturation of labeled cells. Immunohistochemistry of cells positive for EDU+ and oligodendrocyte linage marker Olig 2 (A), the mature oligodendrocyte marker CAII (B) or the OPC marker PDGFR A (C) were quantified in fimbria and show an increase in $\mathrm{EDU}^{+}$Olig 2+ and EDU ${ }^{+}$PDGFR $\mathrm{A}^{+}$double positive cells $\left(\mathrm{A}^{\prime}, \mathrm{C}^{\prime}\right)$. (D-E) The densities of Olig 2, CAII and PDGFR A+ cells were increased $46 \mathrm{~W}$ pti (D) while the fimbria area was not significantly decreased (E). (F) Quantification of CAII ${ }^{+}$cells at $26 \mathrm{~W}, 46 \mathrm{~W}$ and $52 \mathrm{~W}$ pti revealed a consistent increase in $\mathrm{CAII}^{+}$cells at after adult MBP iKO. (A', B', C': Percentage of EDU positive cells from Olig 2/CAII or PDGFRA positive cells per FOV) N= 4 animals, two-tailed unpaired $t$-test; $p<.05\left(^{*}\right), p<.01\left(^{* *}\right), p<.001\left(^{* * *}\right)$, scale bar $50 \mu \mathrm{m}$.

From these data showing that oligodendrocyte numbers are not reduced, but proliferation rate and numbers are slightly increased we concluded that the induced $M b p$ ablation and the resulting loss of MBP do not cause widespread oligodendrocyte death. MBP is not required for survival in mature oligodendrocytes that differentiated in the presence of MBP before the induced knock-out.

\subsection{Cholesterol synthesis is affected on tissue level but not in oligodendrocytes}

The corpus callosum is another well-defined myelin tract in the CNS and was previously chosen for Lipidome analysis. Determination of astrocytic, microglial and oligodendrocyte genes was performed to assess demyelination and inflammation. The kinetics of myelin loss characterized by the decrease in myelinating profiles appeared slower in corpus callosum therefore late time points at $40 \mathrm{~W}$ and $52 \mathrm{~W}$ pti were chosen for this analysis. Both time points showed an increase in the microglial markers lysosomal associated membrane protein 2 (Lamp 2) and allograft inflammatory factor 1 (Aif1) as well as in astrocyte markers such as glial fibrillary acidic protein (Gfap), aquaporin 4 (Aqp4) and S100 calcium binding protein B (S100b) which are evidence for microgliosis and astrogliosis (Figure 3-28, A) A reduction of MBP expression by approximately $50 \%$ together with an increased expression of carbonic anhydrase 2 (Car2), platelet derived growth factor receptor alpha (Pdgfr $A$ ) and Plp1 is indicative for continuous presence of recombined oligodendrocytes as well as an increased number of OPCs. (Figure 3-28, B). 

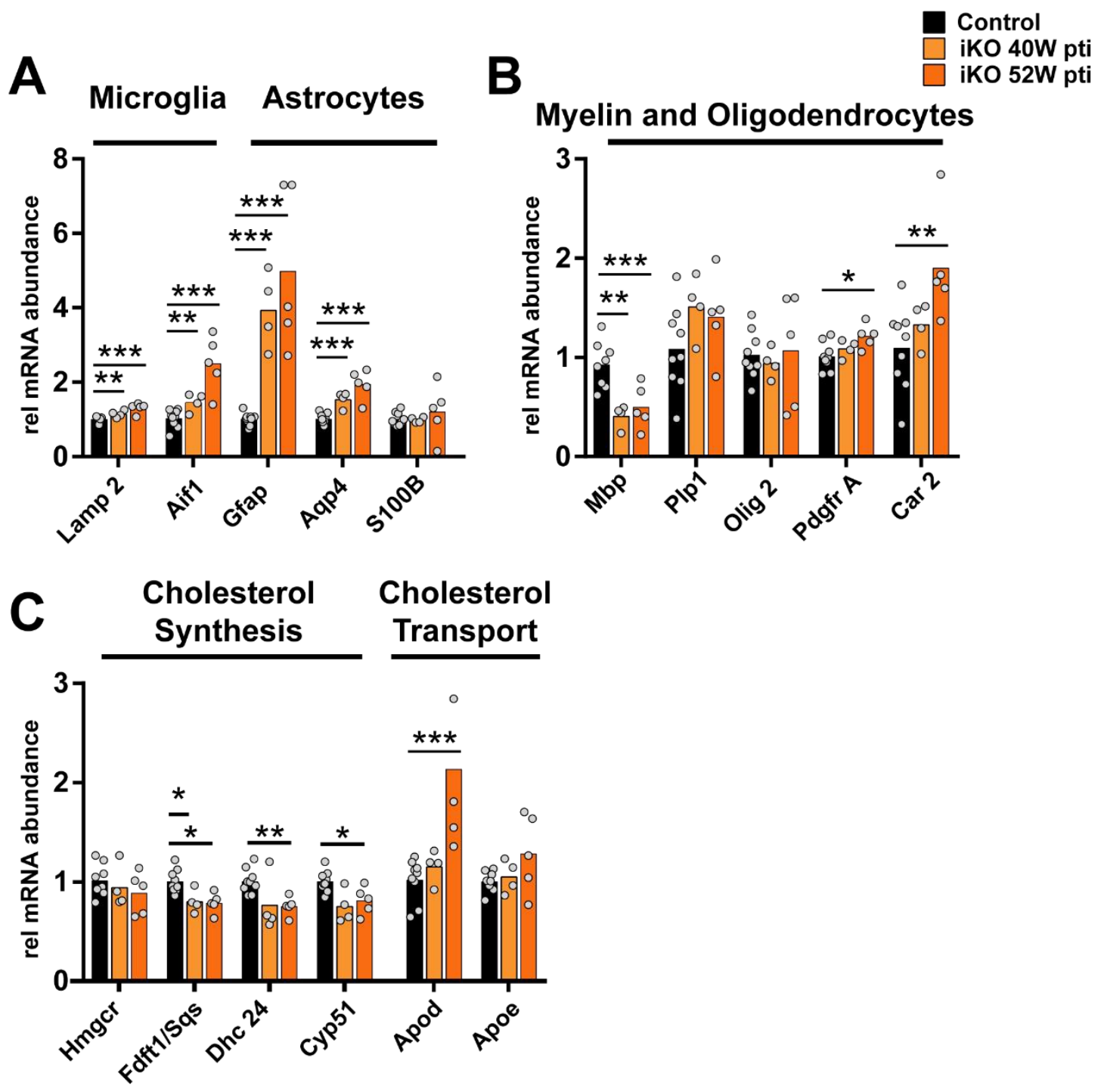

Figure 3-28 Decreased cholesterol synthesis in corpus callosum 52W pti

(A) qRT-PCR analysis of corpus callosum $40 \mathrm{~W}$ and $52 \mathrm{~W}$ pti reveals an increase in astrocyte and microglial markers (B) Expression of $M b p$ mRNA is reduced to 50\% of control while the expression of PLP and Olig 2 is unchanged. The OPC marker PDGFR A and the mature oligodendrocyte marker CAII show increased expression at 52W pti. (C) Expression of genes involved in cholesterol synthesis are decreased by approximately $20 \%$ while Apod, a gene involved in cholesterol transport is increased $52 \mathrm{~W}$ pti. Controls from $40 \mathrm{~W}$ and $52 \mathrm{~W}$ pti were pooled, expression was normalized to Rps13 and Rplp0, N=4-5 animals, two-tailed unpaired $t$-test.

The synthesis of cholesterol is an essential and rate limiting step for myelination and ablation of cholesterol synthesis in oligodendrocytes leads to delayed CNS myelination (Saher et al., 2005). Lipidome results showed only minor reduction in cholesterol abundance after ablation of $M b p$. To assess whether cholesterol synthesis was reduced potentially affecting normal steady state myelin maintenance we tested for changes in genes belonging to the cholesterol synthesis pathway. While the expression of the rate limiting enzyme 3-hydroxy-3-methylglutaryl-CoA reductase ( $\mathrm{HmgCr}$ ) was not significantly changed other genes involved in cholesterol synthesis such as squalene synthase ( $F d f t 1), 24-$ dehydrocholesterol reductase (Dhc24) and cytochrome P450 Family 51 (Cyp51) were decreased in expression by approximately $20 \%$. At the same time cholesterol transport 
genes such as apolipoprotein D (Apod) showed and increased expression 52W pti while apolipoprotein E (Apoe) was unchanged (Figure 3-28, C).

To differentiate whether the decreased expression of cholesterol synthesis genes was attributed to oligodendrocytes magnetic cell isolation (MACS) of 04 positive cells from corpus callosum 16W pti was performed in collaboration with Lena Spieth, Stefan Berghoff and Tim Düking (AG Dr. Gesine Saher, Max-Planck-Institute for Experimental Medicine). For expression analysis RNA was extracted from isolated cells and cDNA amplification by single primer isothermal amplification (SPIA) was performed and followed by qRT-PCR (Figure 3-29, A). The expression of myelin and oligodendrocyte genes other than $M b p$ was not reduced in isolated oligodendrocytes (Figure 3-28, B) with Mag showing a trend towards increased expression that is consistent with expression analysis on whole brain lysate (Figure 3-3). Analysis of genes involved in cholesterol biosynthesis revealed no significant change in expression of sterol regulatory element-binding protein 2 (Srebp2), Hmgcr, Fdft1, Shc24, Cyp51 and Fdps (Figure 3-28, C). Furthermore, genes involved in cholesterol including Apoe, Apod and low density lipoprotein receptor ( $L d l r)$ were not changed in isolated oligodendrocytes (Figure 3-28, D). From these data it can be concluded that in oligodendrocytes the expression of major cholesterol synthesis genes is not affected by $M b p$ ablation.
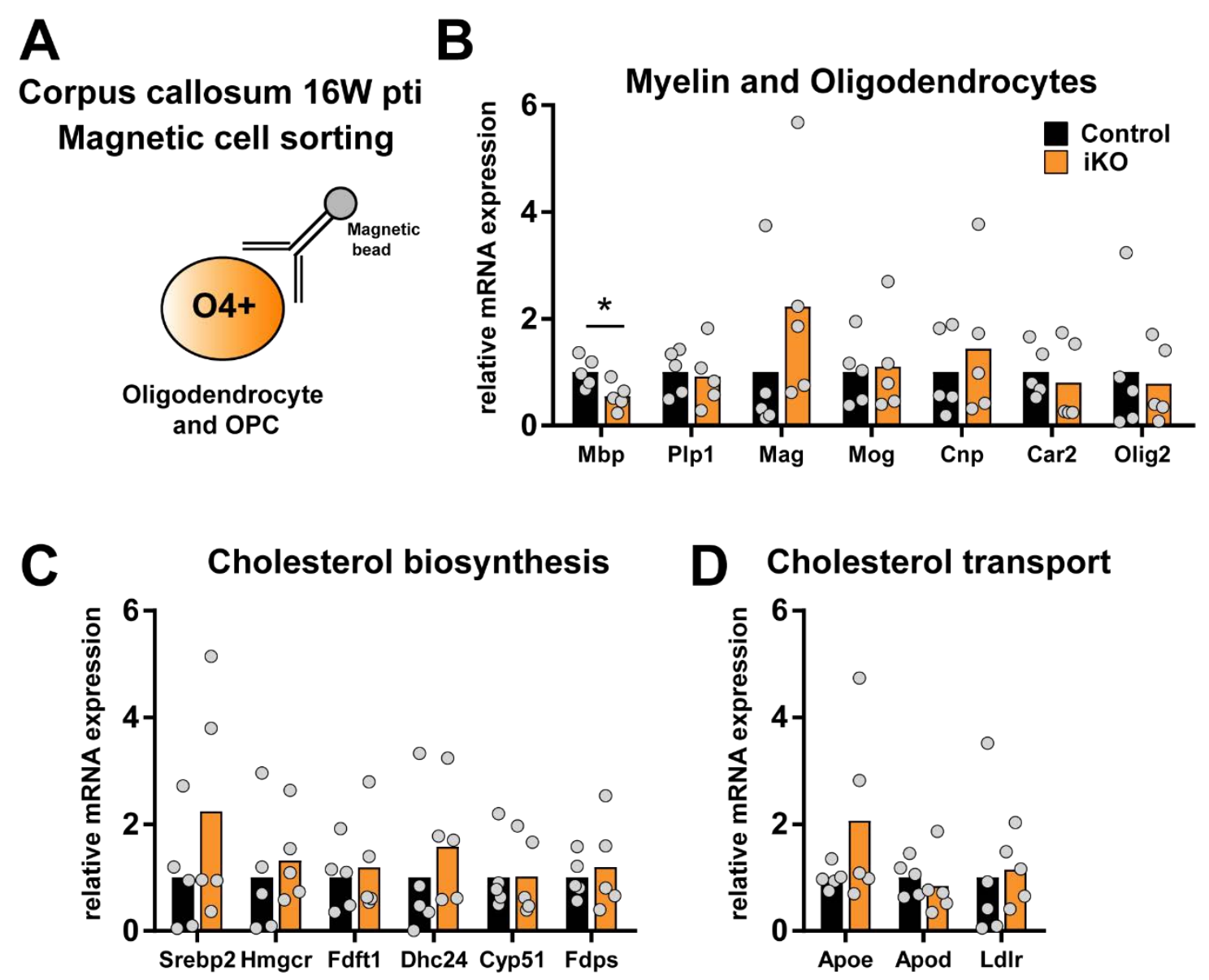

D Cholesterol transport

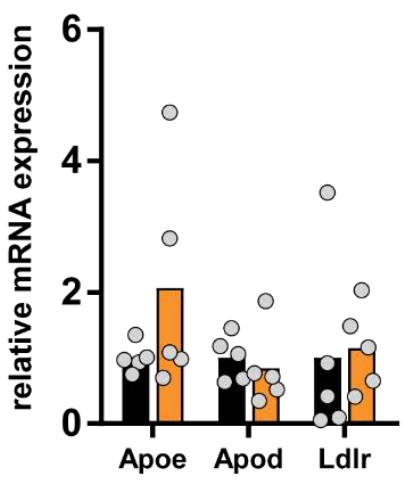

Figure 3-29 Isolated 04+ cells show no decrease in cholesterol synthesis genes

(A) Magnetic cell sorting and isolation of $\mathrm{O}^{+}$cells including oligodendrocytes and OPCs from corpus callosum of mice $16 \mathrm{~W}$ pti. Isolation was performed in collaboration with Lena Spieth, Stefan Berghoff and Tim Düking (B) qRT-PCR analysis reveals that isolated $\mathrm{O4}^{+}$cells have a reduced $\mathrm{Mbp}$ expression but not significantly altered expression of other myelin and oligodendrocyte proteins. (C) Major proteins of the cholesterol synthesis 
pathway are unchanged with a tendency to increased expression in $04^{+}$cells. (D) Cholesterol transport genes in $04+$ cells were not significantly changed. $\mathrm{N}=5$ Corpora callosa obtained from individual animals, expression was normalized to Rps13 and Rplp0 used as housekeepers , two-tailed unpaired $t$-test.

To summarize, the inducible MBP knock-out mouse model fulfills all requirements to study myelin maintenance and turnover in the adult CNS. Low levels of inflammation, absence of widespread oligodendrocyte death and ongoing synthesis of cholesterol synthesis genes as well as myelin genes allows to study ongoing myelin turnover in a mostly unperturbed state. Reductions in myelin proteins, myelinated profiles and compact myelin thickness indicated a slowly progressive loss of compact myelin.

\subsection{Internodal shortening after MBP iKO}

To understand the replacement of compact myelin by tubular membranes and the increased number of unmyelinated axons we investigated the structure of individual myelin internodes. 2D TEM cross sections allow to study changes in the axonal population by quantification of many axons and myelin profiles in nerve cross sections. However, the 2D images does not reveal at which position along the internode the axons were sectioned. The localization of the section plane within the long myelin internode as for example the distance of the section plane to the node of Ranvier cannot be determined by 2D EM. To study changes in morphology along internodes of individual axons we used 3D volume electron microscopy (FIB-SEM).

$3 \mathrm{D}$ reconstruction and visualization revealed that the observed inner tongue membrane processes are tube-like structures connected to the myelin sheath that can extend several $\mu \mathrm{m}$ away from their axon (Figure 3-30, A, yellow arrow heads). Reconstruction also revealed that the percentage of the surface of the axons covered by myelin is reduced after ablation of MBP to approximately $75 \%$ at $16 \mathrm{~W}$ pti and $30 \%$ at $26 \mathrm{~W}$ pti (Figure $3-30, \mathrm{C}$ ). This is also reflected by a progressive reduction in myelinated profiles as quantified on transmission electron micrographs of optic nerve cross sections $8 \mathrm{~W}, 16 \mathrm{~W}, 26 \mathrm{~W}, 40 \mathrm{~W}$ and $52 \mathrm{~W}$ pti (Figure $3-36, \mathrm{~B})$. These data show that myelin in the optic nerve disappears by shortening of the internodes leaving naked axons behind after ablation of MBP in mature oligodendrocytes. Using the density of myelinated axons on a cross section of the optic nerve, we estimated the time after which $50 \%$ of the myelin profiles were lost showing a shortening of the internode by half. The following calculations represent estimates based on the assumption that the shortening of myelin internodes underlies a linear reduction through a shortening or retraction process. At $8 \mathrm{~W}$ pti the number of axons between control and iKO did not change. At $16 \mathrm{~W}$ pti the number of myelinated axons was reduced by $46 \%$ compared to controls and at $26 \mathrm{~W}$ and $40 \mathrm{~W}$ pti by $80 \%$. This suggests that most myelin sheaths belonging to recombined oligodendrocytes were shortened or have vanished by $26 \mathrm{~W}$ pti. The remaining myelinated profiles likely belong mostly to non-recombined oligodendrocytes. A linear regression of the density of myelinated axons in the optic nerve for time points $8 \mathrm{~W}$ $(100 \%), 16 \mathrm{~W}(54 \%)$ and $26 \mathrm{~W}(20 \%)(\mathrm{y}=-4.604 * \mathrm{X}+137.5)$ was calculated. Extrapolating from this regression we found that a level of $50 \%$ of myelinated profiles compared to controls is reached around 19W or 133 days pti. Using FIB-SEM 3D Data we found the coverage of individual axons to be approximately $72 \%$ at $16 \mathrm{~W}$ compared to $96 \%$ in controls and $28 \%$ at $26 \mathrm{~W}$ compared to $92 \%$ in controls. Based on linear regression $\left(\mathrm{Y}=-4,3^{*} \mathrm{X}+\right.$ $141,8) 50 \%$ myelin coverage was reached approximately 21 weeks or 147 days pti. These approximations suggest that the loss of MBP synthesis results in shortening of myelin internodes to $50 \%$ within $133-147$ days after knock-out induction. 

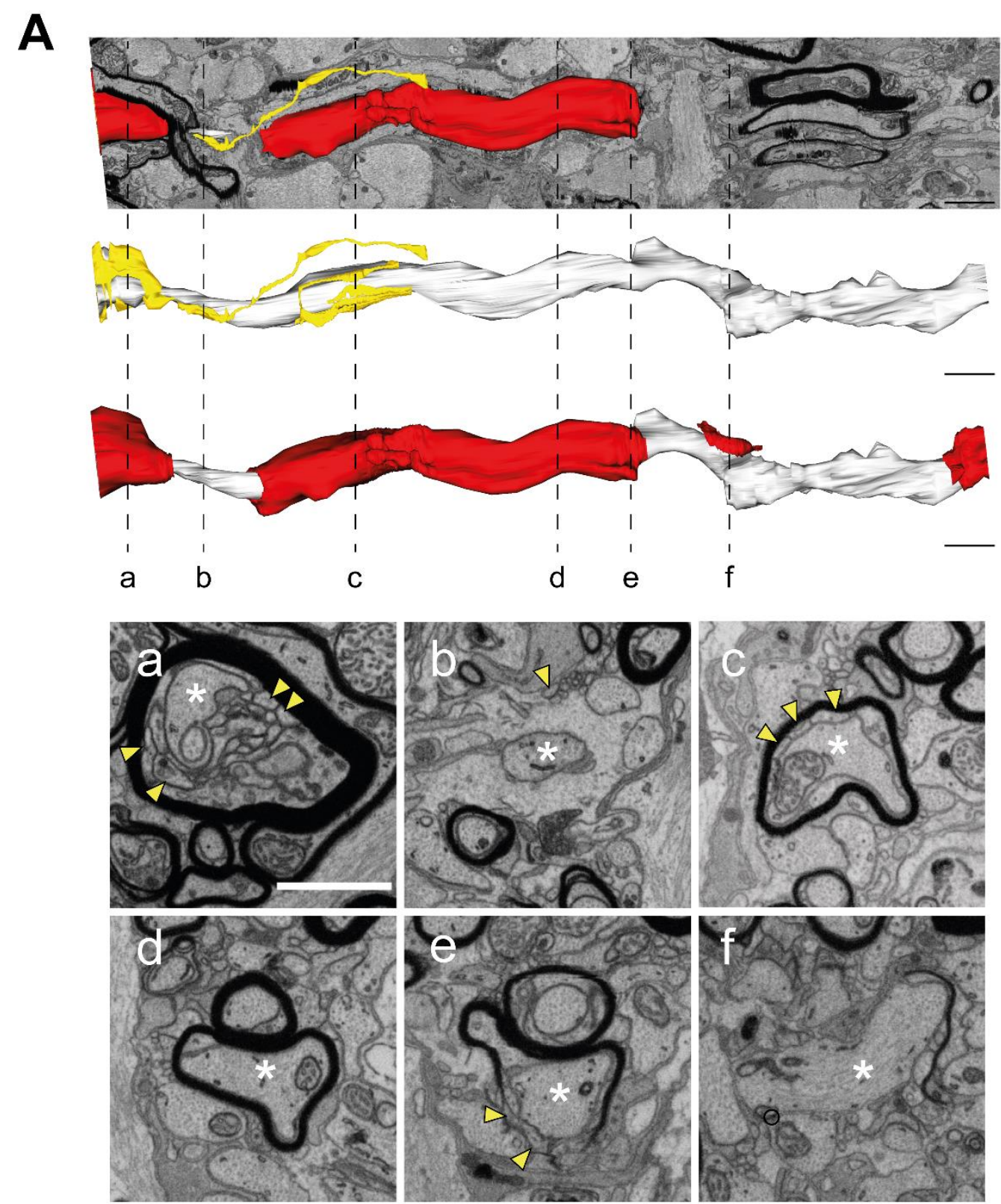

\section{B}

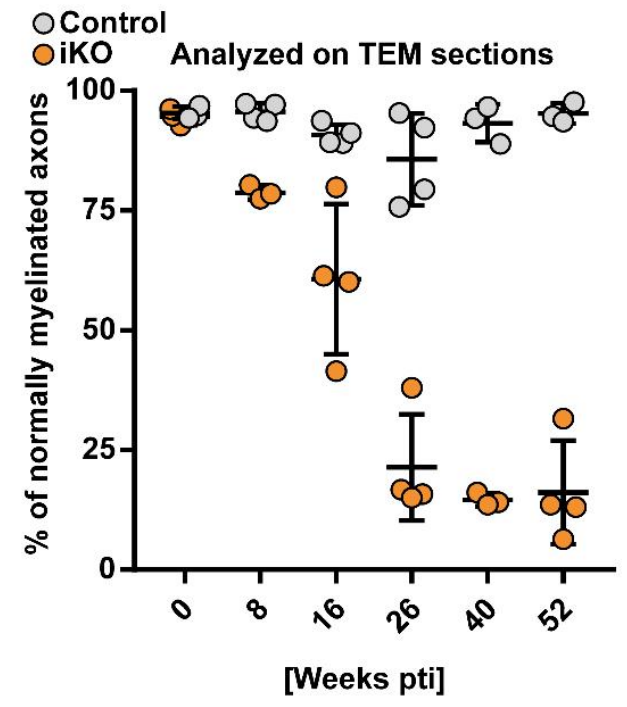

C Analyzed on 3D EM volumes

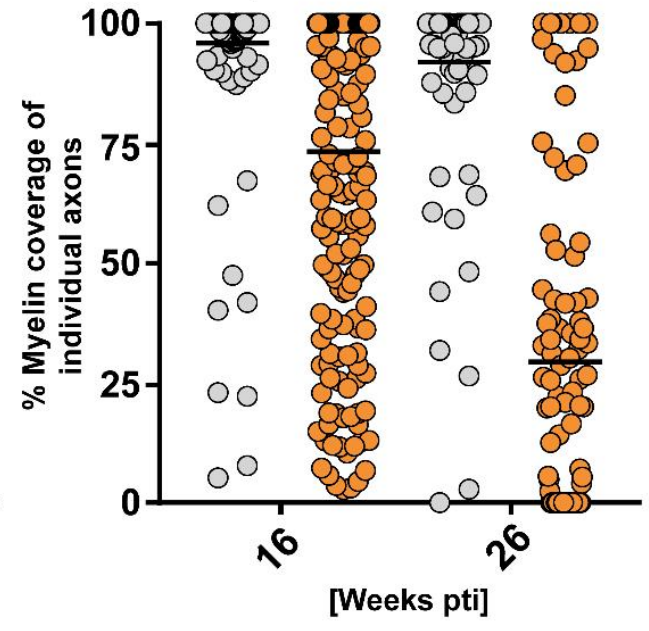


Figure 3-30 Internodal shortening after ablation of MBP

(A)3D reconstruction of a $26 \mathrm{~W}$ pti optic nerve volume acquired by focused ion beam scanning electron microscopy (FIB-SEM) reveals shortening of internodes and tubule like structures of the observed inner tongue phenotype. Non-compact myelin tubules (yellow arrowhead), reconstructed axon (white asterisk). (C) The percentage of myelin covered axonal length decreases after MBP iKO to approximately $75 \%$ at $16 \mathrm{~W}$ pti and $30 \%$ at 26W pti. Quantification of one 3D volume per animal at $16 \mathrm{~W}$ pti ( $\mathrm{N}=2$, animals) and 26W pti ( $\mathrm{N}=1$, animals) with $>90$ axons per animal. (B) Transmission electron micrograph analysis of optic nerve reveals a decrease in profiles of normally myelinated axons that reflects the internodal shortening as seen in C. Analysis was performed on cross sections in the retinal part of the optic nerve of at least 5 random sampled micrographs with a total area of $>330 \mu \mathrm{m}^{2}$ and $>200$ axons. $\mathrm{N}=3-4$ animals (parts of the data are already used in Figure 3-10), scale bar $2 \mu \mathrm{m}$. 3D data acquisition was performed by Dr. Anna Steyer, electron microscopy core unit, MaxPlanck-Institute for Experimental Medicine.

\subsection{Non-compact membrane tubules appear preferentially at the juxta-paranode}

To localize sites of myelin turnover within a myelin sheath is challenging and requires appropriate means of visualization and ultrastructural resolution. The mouse line used in this thesis allows the visualization of the absence of MBP by the lack of myelin compaction. Since we found membrane tubules at the inner tongue of the myelin sheath by thin section EM analysis, we aimed to determine the site of emergence of the membrane tubules within a myelinated internode. We assume that the tubules are the result of continued membrane synthesis of the intact oligodendrocyte and therefore indicate the site of active membrane insertion into the myelin sheath. To achieve this, 3D FIB-SEM microscopy of high pressure frozen and freeze substituted optic nerve $16 \mathrm{~W}$ pti was performed. The preparation by high pressure freezing and freeze substitution allows the visualization of the observed membrane structures close to the native state by minimizing fixation and dehydration artefacts (Schertel et al., 2013). 3D representation and reconstruction of an exemplary axon (purple) revealed an accumulation of membrane and non-compact myelin tubules (orange) (Figure 3-31, A $3.5 \mu \mathrm{m}$ ) as well as axonal deformation (Figure 3-31, A 2.5 $\mathrm{m}$ ) at the juxtaparanode close to the node of Ranvier (Figure 3-31, A $0 \mu \mathrm{m}$ ). Approximately in $10 \mu \mathrm{m}$ distance from the node of Ranvier into the internode no membrane accumulation was observed (Figure 3-31, A, $12.5 \mu \mathrm{m}$ ). This phenotype is also observed in longitudinal sections of high pressure frozen optic nerve (Figure 3-31,B). We conclude that the emergence of shiverer-like membrane tubules at the juxtaparanode induces the "myelin stack" formation, internode shortening and subsequent impairment of the nodal structure. In accordance the number of functional node of Ranvier as quantified by labeling of focally enriched NaV 1.6 ion channels flanked by contactin associated protein 1 (CASPR1) is significantly reduced in optic nerve $16 \mathrm{~W}$ and 26W pti (Figure 3-31, C). 
Results

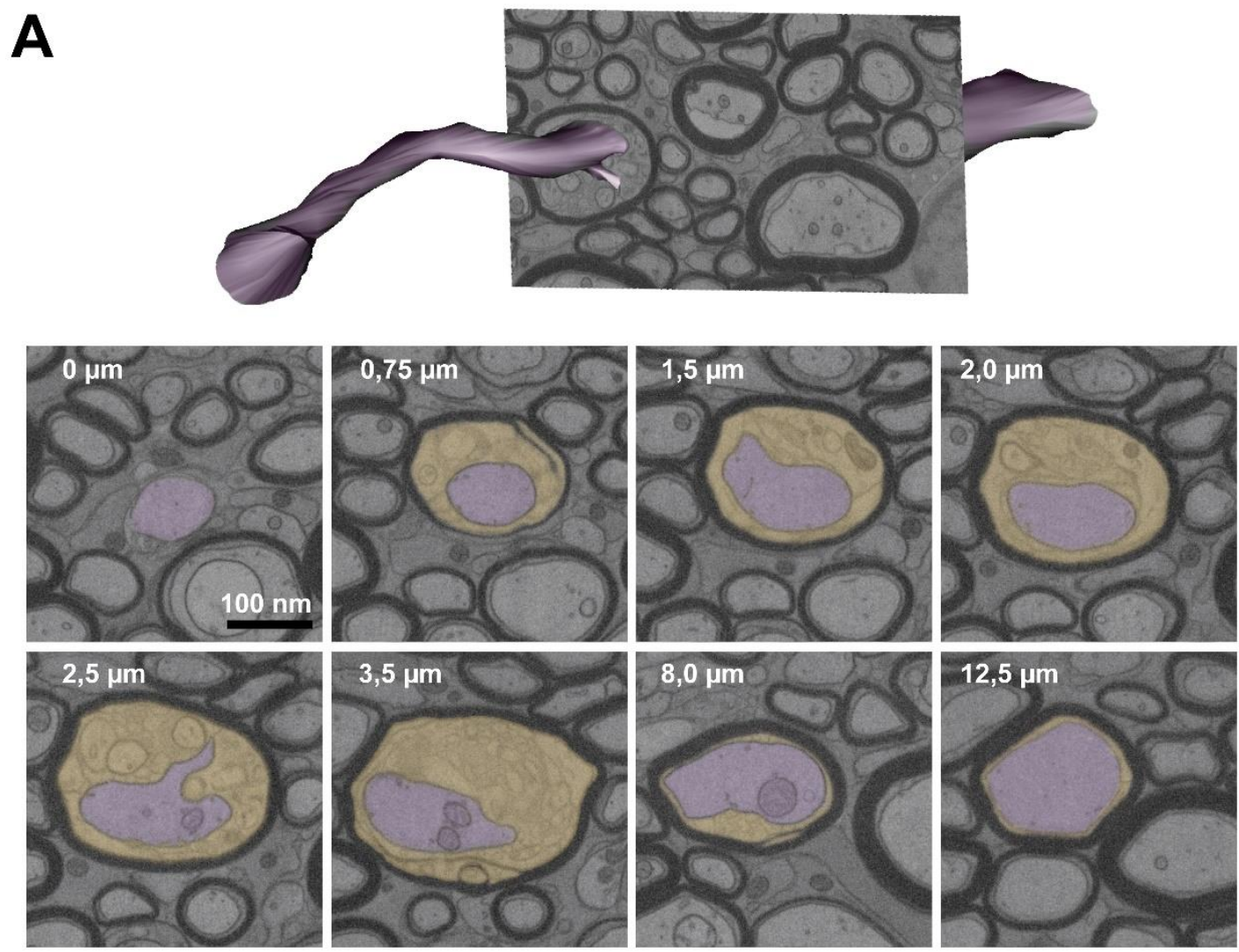

\section{B}

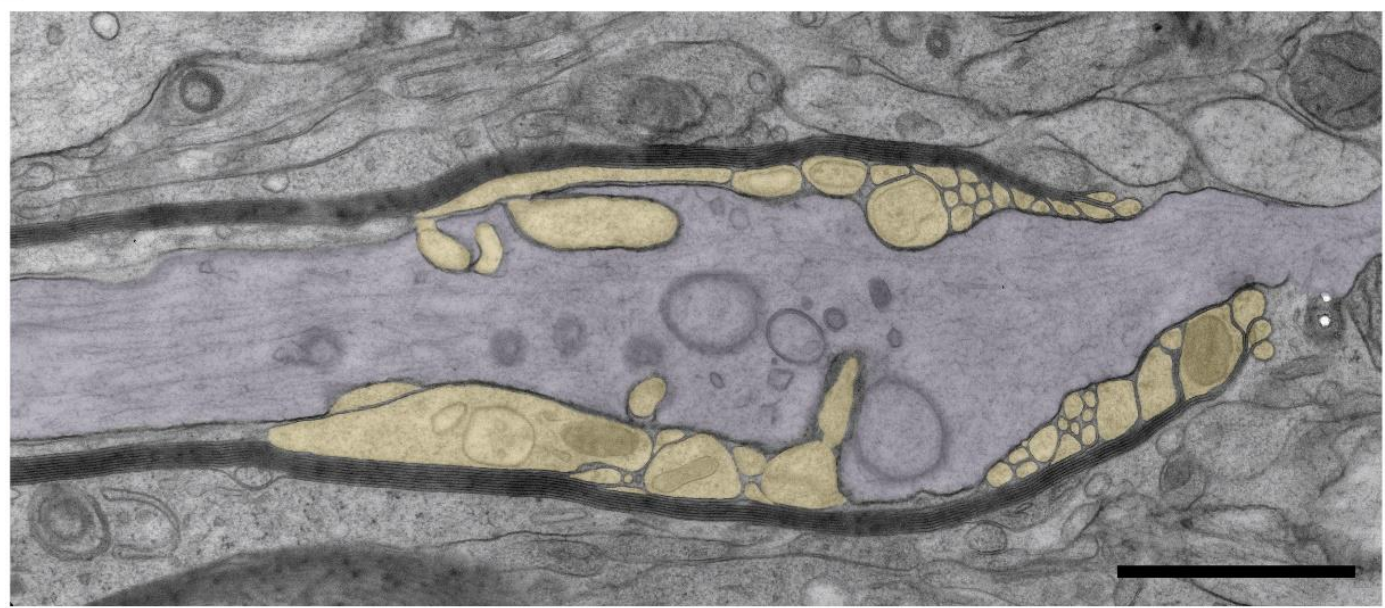

C
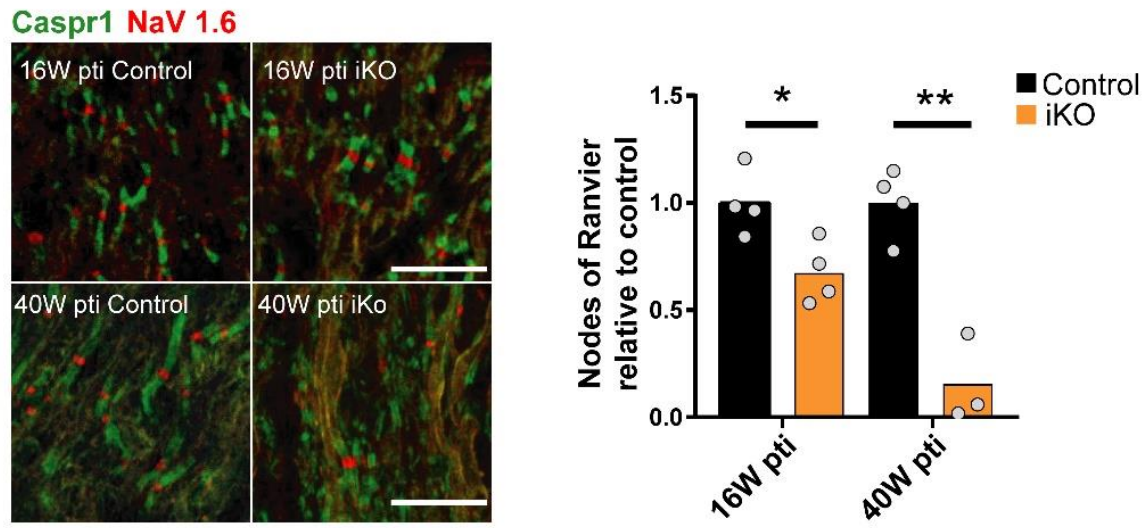
Figure 3-31 Non-compact membrane tubules appear preferentially at the juxta-paranode

(A) Segmentation of a myelin internode reveals that a massive accumulation of non-compact tubules occur predominantly at the juxta-paranode $(1,5-3,5 \mu \mathrm{m})$ while the same axons seems unaffected further along the internode $(12,5 \mu \mathrm{m})$ (High pressure frozen and freeze substituted optic nerve 16W pti.). (B) High resolution visualization of the juxta-paranodal phenotype on transmission electron micrographs of a longitudinal sectioned juxta-paranodal region reveals large amounts of non-compact membrane and detachment of paranodal loops close to the node of Ranvier (High pressure frozen and freeze substituted optic nerve 26W pti) (C) The loss of functional nodes of Ranvier as demonstrated by loss of NaV 1.6 and CASPR1 signal can be observed 16W and $40 \mathrm{~W}$ pti. (confocal images of longitudinal optic nerve cryosections $\mathrm{N}=3-4$ animals, two-tailed unpaired $t$-test.

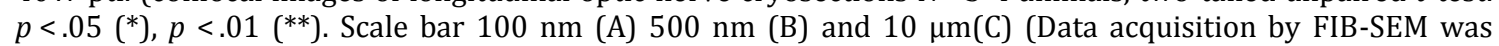
performed by Dr. Anna Steyer, Electron microscopy core unit Max-Planck-Institute for Experimental Medicine).

\subsection{Increase in myelin spheres at the inner tongue}

As shown above, the internodal shortening can be explained by the insertion of MBP-free membrane tubules and the loss of axo-glia interaction at the paranode. These tubules are representing myelin renewal. For the observed thinning of the myelin sheath removal mechanisms must play a role. Myelin removal during aging and demyelination is partially mediated by uptake trough astrocytes (Ponath et al., 2017) and microglia (Safaiyan et al., 2016). In our model of genetic demyelination we found long myelin outfoldings (Figure 3-32, A, A') and myelin debris close to and in astrocytic processes (Figure 3-32, B, B'). Also microglial are found in direct contact to myelin protrusions and non-compact myelin membrane. The presence of dark degradative compartments containing myelin debris show microglial uptake of myelin (Figure 3-32, C, C'). Beside myelin removal by astrocytes and microglia, the shedding of myelin spheres (Hill et al., 2018) or Marchi bodies (Hildebrand and Aldskogius, 1976; Persson et al., 1992) are discussed as a mechanism of myelin disposal.

When analyzing our mouse model, a progressive decrease in myelin thickness as measured by corrected g-ratio in optic nerve was observed at $16 \mathrm{~W}$ and $26 \mathrm{~W}$ pti as described above (Figure 3-12, C, D and Figure 3-33, A). At the same time an increased number of myelin spheres at the inner tongue of oligodendrocytes was found. These structures resemble compact myelin membrane that is either unconnected or only partially connected to the myelin sheath (white arrow head in panel (e)) (Figure 3-33, B). These myelin spheres are localized in the inner tongue along the axon and appear in control as well as iKO animals (Figure 3-33, C, D). Using 3D FIB-SEM volume imaging the abundance of myelin spheres per $\mu \mathrm{m}$ axonal length was quantified and found to be significantly increased in optic nerve $16 \mathrm{~W}$ and $26 \mathrm{~W}$ pti (Figure 3-33, D). Since the observed myelin spheres are a rare event and due to a limited 3D volume that did not always cover complete internodes, most analyzed axons were lacking myelin spheres. To perform a statistical analysis the data from 16W and 26W were pooled and displayed as averages of individual animals revealing a significant increase in the number of myelin spheres per $\mu \mathrm{m}$ axonal length (Figure 3-33, E). 

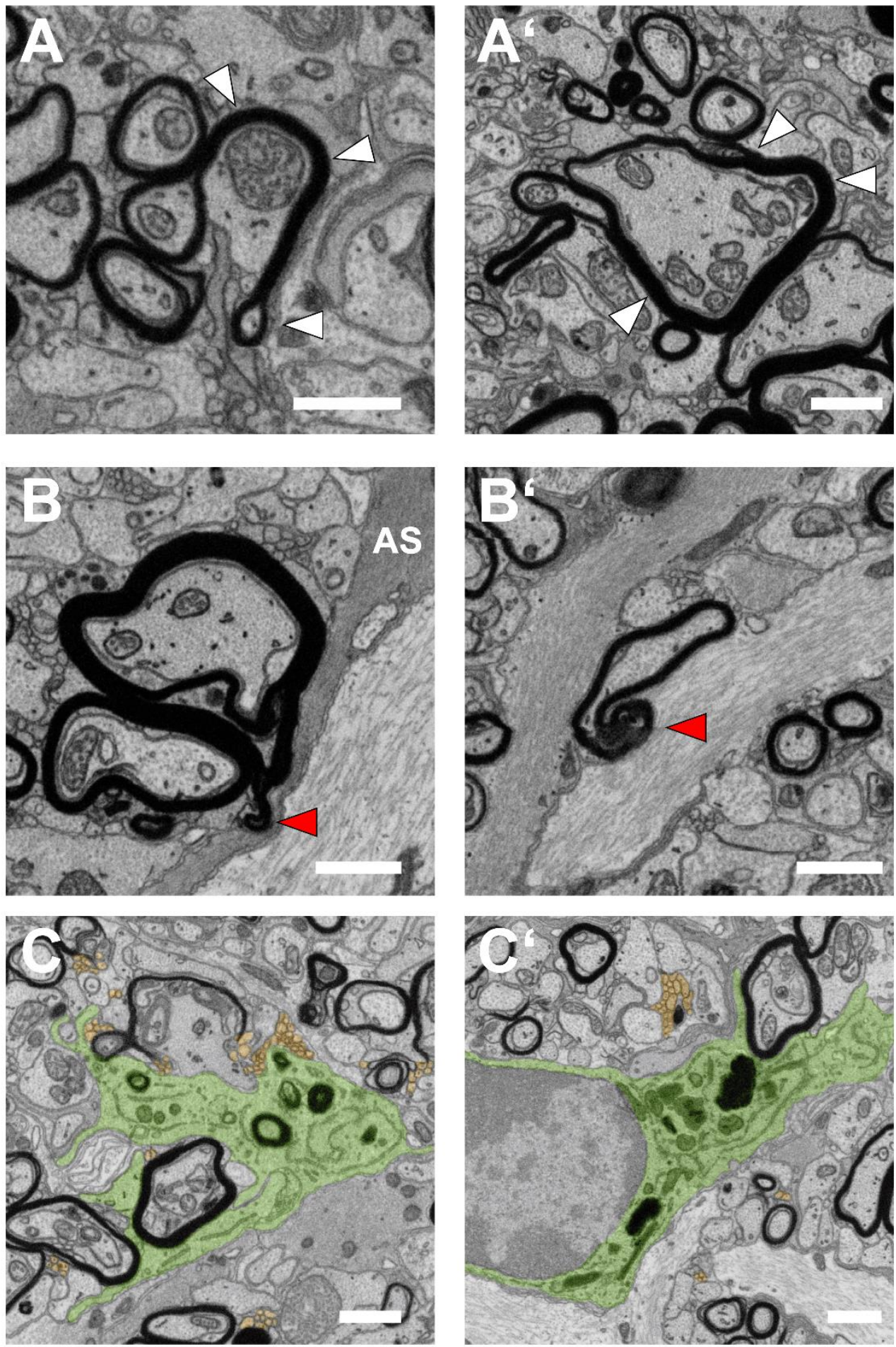

Figure 3-32 Microglia and astrocytes take up myelin

(A, A') Myelin outfoldings (white arrow head) are detectable and significantly increased. (B-B') Astrocytes (AS) are found in direct contact to myelin protrusions (red arrow head). (C-C') Microglia (false colored in green) are found close to demyelinating axons (Asterisk) and non-compact myelin membrane (false colored in orange). Presence of dark degradative compartments (Cantuti-Castelvetri et al., 2018) containing myelin debris show microglial uptake of myelin. Images were extracted from a FIB-SEM volume of optic nerve 26W pti, scale bar 1 $\mu \mathrm{m}$. 
A
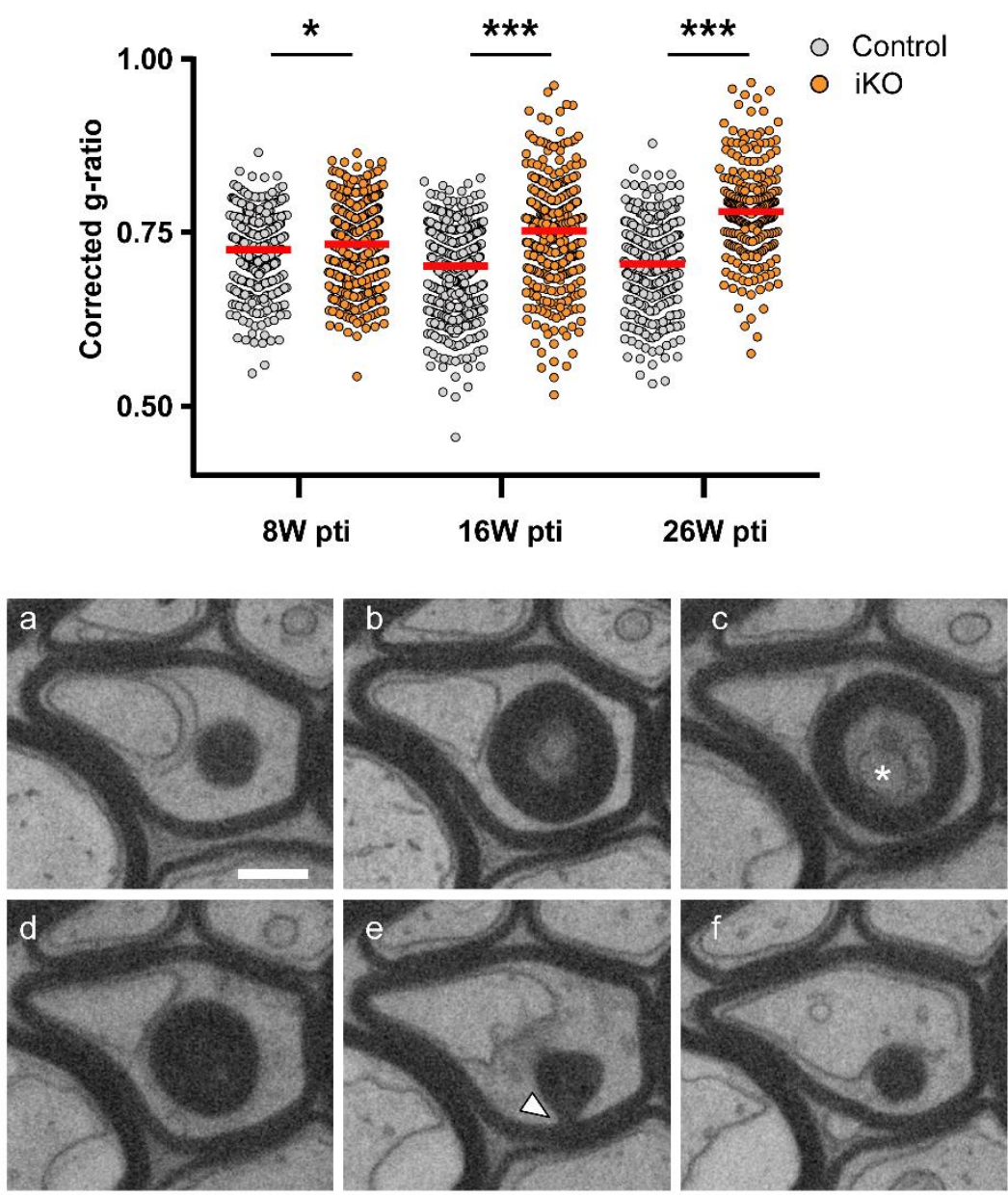

C
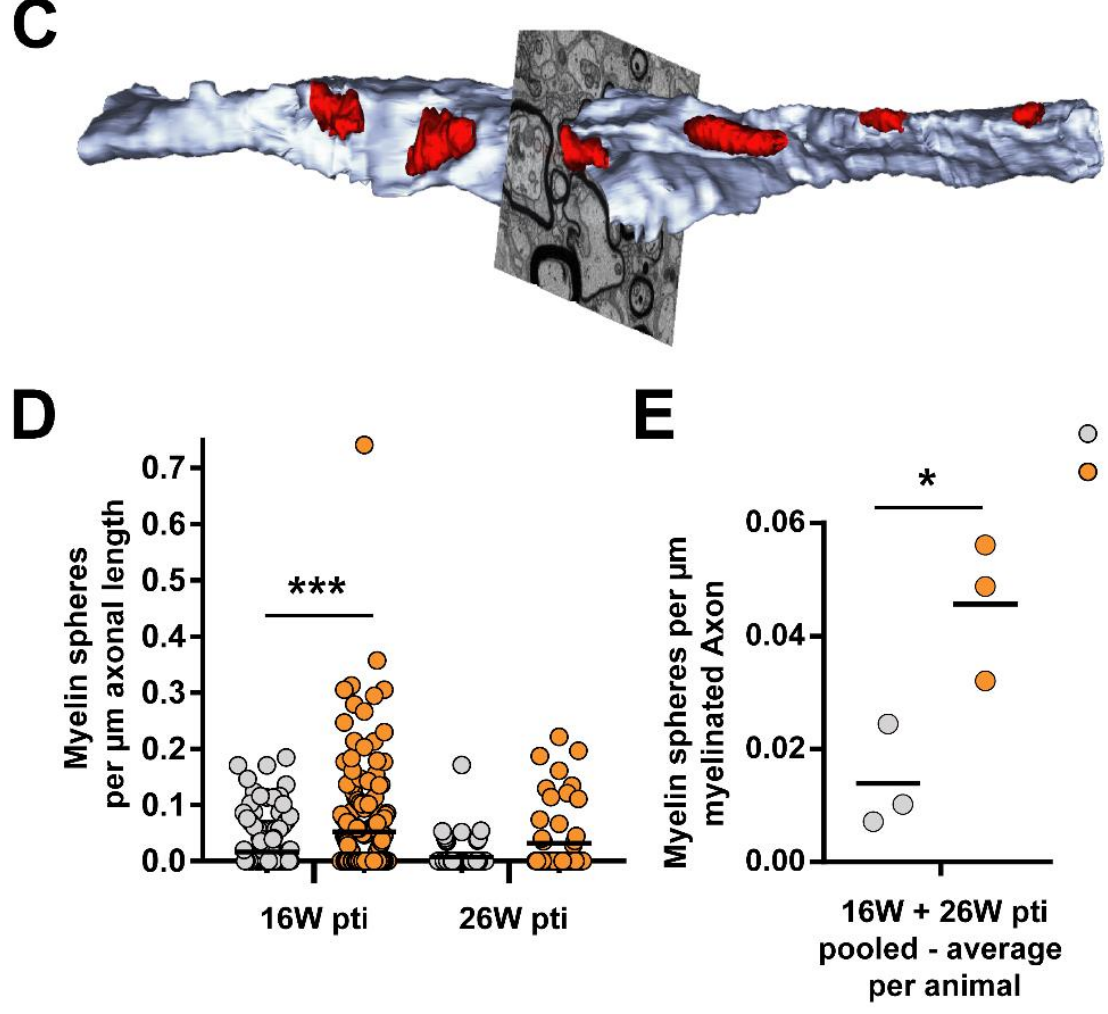
Figure 3-33 Thinning of compact myelin and increased number of myelin spheres at the inner tongue

(A) G-ratio measurements corrected for inner tongue enlargement by subtraction of inner tongue area from the complete fiber reveal progressive thinning of myelin sheaths after MBP iKO data also plotted in Figure 3-12 BD)(>100 axons per animal, N=3-4 animals). (B) 3D electron microscopy (FIB-SEM) reveals compact myelin spheres in the oligodendrocyte inner tongue. (16W pti, high pressure frozen and freeze substituted optic nerve, frame a-f cover a z-volume of $330 \mathrm{~nm}$ ) (C) Spheres of compact myelin in the inner tongue visualized along an axon (optic nerve, 26W pti, K\&S fixation (blue: axon, red: myelin spheres)). (D) The number of inner tongue myelin spheres per $\mu \mathrm{m}$ axonal length is increased in $\mathrm{iKO} 16 \mathrm{~W}$ and $26 \mathrm{~W}$ pti. on the level of individual axons (D) and by averaging the number of myelin spheres per axon per animal (E) Quantification of one 3D volume per animal at $16 \mathrm{~W}$ pti $(\mathrm{N}=2$, animals) and $26 \mathrm{~W}$ pti $(\mathrm{N}=1$ animal) with $>90$ axons per animal. Kolmogorow-SmirnowTest (A and D) and two-tailed unpaired $t$-test (D) $p<.05\left(^{*}\right), p<.01\left(^{* *}\right), p<.001\left(^{* * *}\right)$. FIB-SEM acquisition was performed with the help of Dr. Anna Steyer, Electron microscopy core unit Max-Planck-Institute of Experimental Medicine, scale bar $0.5 \mu \mathrm{m}$.

The g-ratio along an axon can vary considerably as shown by 3D electron microscopy (Giacci et al., 2018). Hence, we decided to investigate a potential link between the thinning of myelin and the localization and presence of myelin spheres along an internode. Five myelinated axons from control and iKO $26 \mathrm{~W}$ pti were chosen for analysis. The corrected gratio was measured every $\mu \mathrm{m}$ along the axon and plotted together with the occurrence of myelin spheres at the inner tongue (red circle) and myelin outfoldings (green circle) (Figure 3-34). While the corrected g-ratio is increased compared to control axons no obvious association between the number of myelin spheres and changes in corrected g-ratio were observed. From these results we conclude that the local occurrence of myelin spheres seems to be mostly independent of the myelin thickness at that position. Interestingly, most spheres were observed at the inner tongue. These spheres are not accessible to removal by microglia or astrocytes. Therefore, we assume that these myelin spheres are indications of oligodendrocyte-intrinsic mechanisms of myelin removal and recycling. 

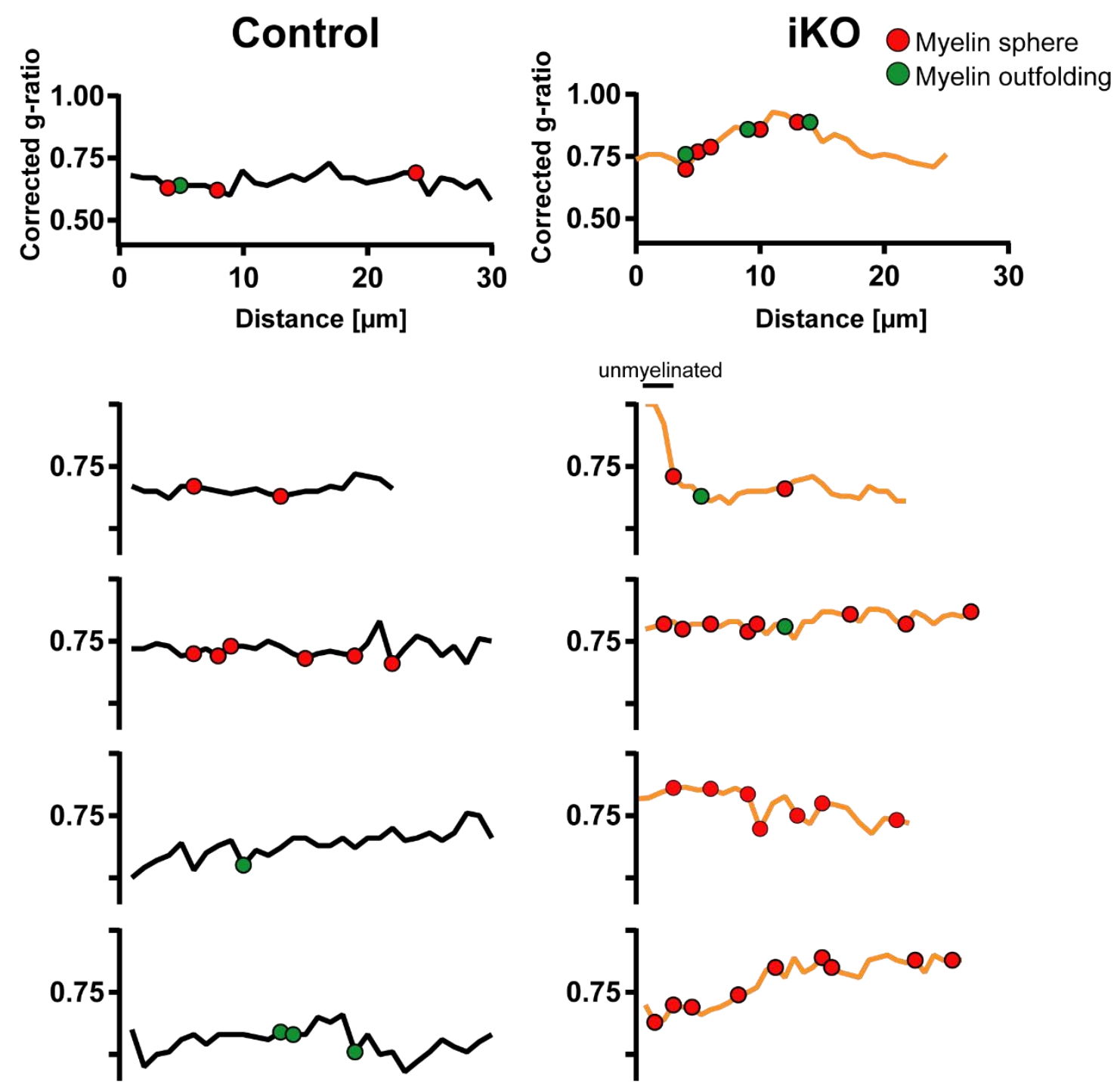

Figure 3-34 Visualization of myelin spheres at the inner tongue

The presence of myelin spheres (red circles) or myelin outfoldings (green circles) have no obvious effect on the corrected g-ratio. Corrected g-ratio was sampled every $\mu \mathrm{m}$. Five axons from control and iKO were measured within one FIB volume per condition in optic nerve $26 \mathrm{~W}$ pti. 


\subsection{Signs of remyelination 52 weeks after knock-out induction}

Since the tremor of knock-out mice showed a peak in severity at $40 \mathrm{~W}$ pti followed by a decline in tremor score we aimed to test for signs of remyelination that could explain the decreasing motor impairment. qPCR analysis of $M b p$ and Plp transcripts at 5 time points for brain and 4 time points for spinal cord from $8 \mathrm{~W}$ to $52 \mathrm{~W}$ pti were performed and revealed significant changes in Mbp and Plp1 mRNA expression. While expression of Mbp mRNA was significantly reduced in iKO between $8 \mathrm{~W}$ and $26 \mathrm{~W}$ pti it showed a progressive trend to increase in both tissues. Comparing knock-out animals $8 \mathrm{~W}$ pti to knock-out animals $52 \mathrm{~W}$ pti revealed increased $M b p$ mRNA levels in brain and spinal cord that were no longer significantly different from control (Figure 3-35, A, B). For Plp1 the relative expression in iKO brain is unchanged between $8 \mathrm{~W}, 16 \mathrm{~W}, 26 \mathrm{~W}$ and $40 \mathrm{~W}$ pti and shows a significant increase at $52 \mathrm{~W}$ pti. In contrast the abundance of Plp1 in the spinal cord compared to control is significantly decreased at $8 \mathrm{~W}, 16 \mathrm{~W}$ and $26 \mathrm{~W}$ and unchanged with a tendency to an increase at $52 \mathrm{~W}$ pti. From the increased $M b p$ and Plp1 transcript abundance in brain and spinal cord at $52 \mathrm{~W}$ compared to $8 \mathrm{~W}$ pti we conclude that proliferation of non-recombined OPCs and differentiation to oligodendrocytes as well as remyelination has occurred.

Next, we assessed sings of remyelination in the optic nerve of animals $52 \mathrm{~W}$ pti. Optic nerves were separated in a proximal/chiasmal and a distal/retinal part and corrected g-ratios were obtained (Figure 3-36). It is known that remyelinated axons in the CNS have thinner myelin which is reflected in an increased g-ratio (Blakemore, 1974). We found increased corrected g-ratios in proximal (Figure 3-36, D) and distal (Figure 3-36, C) optic nerve as reflected in an upshift in the cloud of corrected g-ratios compared to control. We compared corrected g-ratio change to control and found it to be higher in the distal part compared to the proximal part of the optic nerve. Hence, we concluded that remyelination was going on in the optic nerve $52 \mathrm{~W}$ pti and due to the presence of compact myelin and $M b p$ transcript in brain and spinal cord has been generated by newly formed non-recombined oligodendrocytes at a very late time point after knock-out induction. 


\section{Brain}

\section{Spinal Cord}

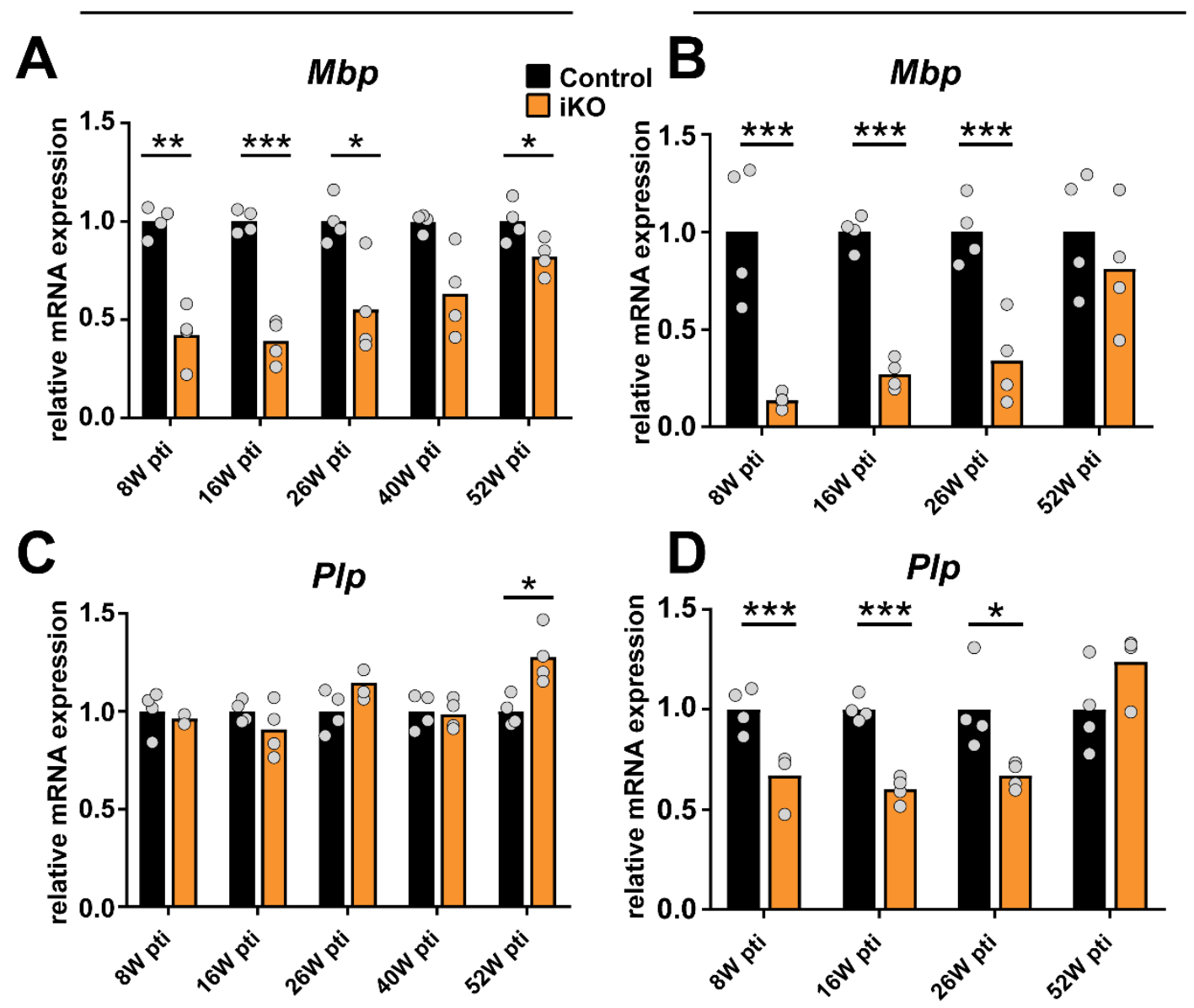

Figure 3-35 Increase in Mbp and Plp mRNA as signs of oligodendrocyte maturation and remyelination

$M b p$ mRNA is strongly reduced in brain (A) and spinal cord (B) after knock-out induction. Abundance of Plp mRNA in Brain is unchanged 8W to 40W pti. but consistently decreased in spinal cord. Despite the loss of $M b p$ mRNA in adult oligodendrocytes, the levels of mRNA increase between $26 \mathrm{~W}$ and $52 \mathrm{~W}$ pti in brain and spinal cord (A-B) which together with increased levels of PLP 52W pti is indicative for maturation of newly formed mature oligodendrocytes (C-D) q-PCR analysis of total brain and spinal cord, N=4 animals, two-tailed unpaired $t$-test; $p<.05\left(^{*}\right), p<.01\left(^{* *}\right), p<.001\left(^{* * *}\right)$. 


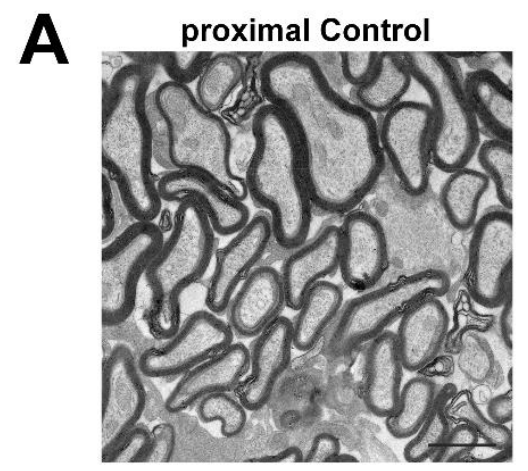

B
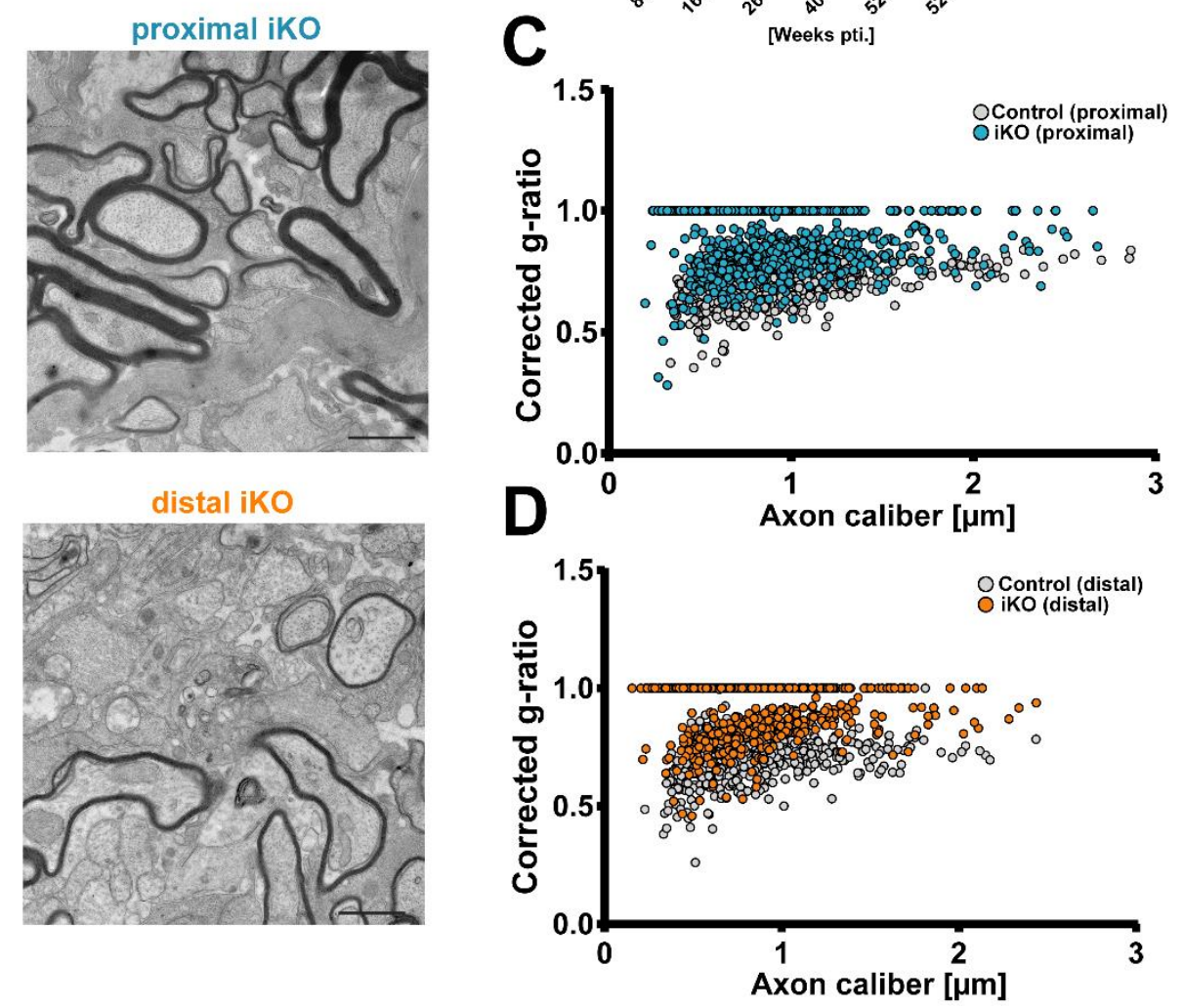

Figure 3-36 Remyelination in the optic nerve $52 \mathrm{~W}$ pti

(A) Electron micrographs show thin myelin sheaths with intact myelin in optic nerve 52W pti. (B) A continuous decrease in myelinated axons is observed between $8 \mathrm{~W}$ and $40 \mathrm{~W}$ pti while at $52 \mathrm{~W}$ pti a tendency for more myelin profiles can be observed in distal and proximal part of the optic nerve (B). N=3-4 animals, two-tailed unpaired $t$-test; $p<.001{ }^{* * *}$ ). (C, D) Corrected g-ratios for proximal (C) and distal (D) parts of the optic nerve $52 \mathrm{~W}$ pti show presence of thinner myelin and a large number of unmyelinated axons with a corrected g-ratio of 1 compared to control. The number of myelinated fibers is larger in the proximal compared to the distal part. $\mathrm{N}=$ 4-5 animals, $>150$ axons per animal. Corrected g-ratio measurements were performed by Daniela Doda as part of a labrotation, scale bar $1 \mu \mathrm{m}$. 


\section{Chapter 4 Discussion}

\subsection{Studying myelin turnover}

The central nervous system demands seemingly opposing characteristics from its cellular systems: stability and adaptation. On the one hand neurons as well as glia require a high level of plasticity to enable learning and timely adaptation to challenges like injury. On the other hand neurons (Morrison and Hof, 1997; Magrassi et al., 2013) as well as oligodendrocytes (Yeung et al., 2014; Tripathi et al., 2017) are very long lived and need to provide stable pathways that facilitate memory formation and connectivity. For oligodendrocytes this means consistent myelination, inhibition of axonal sprouting (Huang, 2005) and metabolic support to axons (Fünfschilling et al., 2012; Saab et al., 2016). The fact that myelin itself is a highly stable structure is reflected in the long protein life-time of the major myelin proteins (Fornasiero et al., 2018) and lipids (Ando et al., 2003).

However, despite the life time of these proteins in the range of weeks to months, the myelin sheath requires maintenance by replacement of aged and dysfunctional proteins. Studies of human oligodendrocytes have shown that the cells survive up to 50 years but that the myelin compartment is undergoing a much faster turnover (Yeung et al., 2014). Due to the dense structure of the compact myelin which excludes most cytosolic proteins (Aggarwal et al., 2011b) it is difficult to conceive how proteins are removed and replaced to allow turnover.

To study myelin maintenance and turnover in the adult animal an inducible model system is required that allows to investigate integration and removal of myelin components at an age when developmental myelination is completed and the transition to adult levels of myelin turnover and maintenance has happened. Necessary requirements for such a model system include survival of oligodendrocytes, ongoing steady-state synthesis of myelin genes and limited levels of inflammation.

Currently available models to study myelin turnover and maintenance are based on rather invasive manipulation of oligodendrocytes like inducible ablation of the myelin transcription factor MYRF (Koenning et al., 2012) or inducible expression of diphtheria toxin (Traka et al., 2010) which are both so impairing that they lead to oligodendrocyte loss. These models only allow to study changes in oligodendrocyte population but are less informative in understanding the steady state turnover within living oligodendrocytes. Other models that affect myelin are toxin-induced demyelination models like the application of cuprizone (Torkildsen et al., 2008) or lysolecithin (Woodruff and Franklin, 1999; Plemel et al., 2018) and immune mediated demyelination models such as EAE (Kornek et al., 2000). These also lead to oligodendrocyte death and massive inflammation and therefore model acute demyelination rather than the steady state turnover.

Another model used to study myelin turnover is an inducible adult PLP knock-out which was recently published (Lüders et al., 2019). The ablation of Plp synthesis in the adult animal resulted in increased number of myelin outfoldings, lamellae splitting and axonal spheroids 10 months after knock-out induction. This showed that continuous PLP expression is required for preservation of white matter integrity (Lüders et al., 2019). However, despite the appearance of myelin pathology due to lack of proper myelin maintenance by loss of PLP expression, this model does not allow to differentiate between aged and newly synthesized myelin by localized ultrastructural changes. In comparison to 
this, the Mbpflox/flox*PIp ${ }^{\text {CreERT2 }}$ model allows the visualization of myelin turnover at the age of up to a year.

Moreover, new advanced imaging tools like volume imaging by focused-ion-beam scanning electron microscopy (FIB-SEM) allow now to study myelin structure not only on thin section level, but also in three dimensions. Such an analytical approach, together with a mouse model that meets our criteria provided all the tools we need to gain a deeper understanding of such a basic but also enigmatic process like myelin maintenance.

\subsection{A new mouse model to study adult myelin turnover}

To address aspects of myelin turnover by fine structural analysis a mouse model was developed to visualize evidence for turnover in adulthood. The Mbpflox/flox*PlpCreERT2 tamoxifen-inducible mouse line described in this thesis makes use of the compacting function of myelin basic protein (MBP) to determine the sites of membrane insertion within individual mature myelin sheaths. We assumed that newly synthetized myelin membrane after the induced knock out of MBP will lack compaction. The localization of such structural changes along the internode was then achieved by 3D FIB-SEM volume imaging and highresolution transmission electron microscopy.

Ablation of MBP synthesis was performed at 8 weeks in young adult animals when developmental myelination in the brain is considered to be mostly complete (Baumann and Pham-Dinh, 2001) and MBP levels stabilized (Delassalle et al., 1981). The time window for the completion of myelin is currently under debate. Recent studies have questioned the completion of myelination at that the age of 8 weeks (Young et al., 2013; Tripathi et al., 2017). Young and colleagues found oligodendrocyte proliferation rates in the nearly fully myelinated optic nerve at 4 months of age that exceeded the rate expected for first-time myelination of naked axons (Young et al., 2013). They suggested that newly differentiated cells might be involved in replacing aged oligodendrocytes. A recent study by Tripathi and colleagues measured oligodendrocyte densities in different CNS tracts between P70 and P605. They found an increased density of $\mathrm{CC} 1^{+}$cells in the corpus callosum between P150 and P605 while the density in spinal cord decreased. In the optic nerve the cell number decreased between P70 and P90, then increased between P180 and P365 before decreasing again between P425 and P605 (Tripathi et al., 2017). These data show ongoing oligodendrogenesis and remodeling in the different CNS tracts on the level of oligodendrocyte population after P60.

However, the induced ablation of MBP in our model takes place only on the level of mature oligodendrocytes and not OPCs. This is validated by the presence of remyelination within one year after knock-out induction. We therefore argue that we only targeted cells that actively express myelin genes including Plp and have already laid down their myelin sheaths during the first 60 days of life while cells differentiated after tamoxifen injection are not affected. It currently remains unknown whether the myelin produced until P60 is different from myelin produced at e.g. one year. Also, ongoing maturation and changes of the myelin sheath remain possible. Our own proteome data in the department (personal communication Dr. Hauke Werner) reveal that age dependent changes in myelin composition are likely. Validation of these age-related differences are of interest and currently under investigation. 
To understand the kinetics of MBP and myelin decay in our mouse model it is important to assess the mRNA half-life of $M b p$ transcript. It is known that $M b p$ mRNA is packed into RNA granules and that these are enriched in the compact myelin fraction (Colman et al., 1982; Kristensson et al., 1986; Ruskamo et al., 2014) where local translation is triggered by phosphorylation of ribonucleoprotein A2 by Fyn kinase (White et al., 2008; Torvund-Jensen et al., 2014). Due to the packaging into granules the $M b p$ mRNA is most likely more stable compared to the mRNA of other myelin genes that are expressed in the soma and transported as proteins. Studies of Plp and $M b p$ mRNA stability in brain slices have shown that the mRNA half-life of Plp is around 5 hours while the $M b p$ mRNA is stable for more than $12 \mathrm{~h}$ (Mathisen et al., 1997). We therefore investigated the MBP decay after knock-out induction. After tamoxifen treatment the abundance of $M b p$ mRNA reached the level of approximately $40 \%$ of control within 20 days after the first injection. This was the maximal achieved reduction showing that most $M b p$ mRNA has decayed in recombined cells, since at later time points comparable expression levels were detected. Remaining mRNA can most likely be attributed to non-recombined oligodendrocytes. This shows that already the MBP mRNA is stable for several days but reached the baseline level at a time point which we consider 0 weeks after tamoxifen.

Other myelin genes such as Mag, Mog or Cnp were not affected by the loss of Mbp expression which is evident for ongoing myelin synthesis. Increased oligodendrocyte density and low levels of apoptotic cells in fimbria together with persistent low levels of $M b p$ transcript in the brain are indicative for the survival of the recombined oligodendrocyte. Widespread loss of oligodendrocytes would reduce the levels of myelin gene transcripts as for example described after adult ablation of myelin regulatory factor (Koenning et al., 2012). Immunohistochemistry of neuropathological markers in brain and spinal cord at 8 and $16 \mathrm{~W}$ pti has shown low levels of astrogliosis and microgliosis compared to demyelinating models characterized by strong inflammation such as cuprizone (Hibbits et al., 2012) or EAE (Benveniste, 1997). While the levels of inflammation are low, ultrastructural changes of the myelin sheath are observed already at $8 \mathrm{~W}$ pti. These observed membrane structures at the inner tongue appear to be similar to oligodendrocyte processes seen in shiverer mice (Rosenbluth, 1980; Möbius et al., 2016). The emergence of this phenotype at such an early time point before the onset of gliosis allows us to study aspects of myelin turnover in a relatively unperturbed system. 


\subsection{Adult myelin turnover - Insertion of new membrane}

Turnover implies the exchange of old material by newly synthetized molecules, which integrate into the existing structure. We could visualize by structural analysis that after $M b p$ ablation the fully developed myelin sheath is slowly and progressively transformed into a shiverer-like phenotype. We hypothesize that this transition happens from inside-out by adaxonal insertion of newly synthetized non-compacted membranes predominantly at the juxtaparanode. The inner tongue at the juxtaparanodal region was enlarged and filled with numerous membrane tubules. The increased occurrence of tubules towards the juxtaparanode implies that the differing amounts of membrane tubules and inner tongue enlargement previously observed by thin section analysis depends on the distance to the node of Ranvier. Only electron microscopic volume imaging and 3D analysis provided the context for fully understanding the spatial characteristics of this phenotype.

It is also conceivable that MBP is selectively removed from myelin membranes at these sites of observed structural changes and that this tubulation is induced by local MBP loss as described in acute demyelination (Weil et al., 2016). Several findings in our study speak against this possibility: In models of acute demyelination oligodendrocyte death and the concomitant rise in intracellular Ca2+ levels induce a conformational change of MBP that causes dramatic myelin decompaction. Such a scenario could be excluded in our mouse model by the lack of according pathology and the very slow development of the membrane phenotype over weeks and months. Furthermore, ongoing synthesis of Plp, Mag, Mog and Cnp in the brain as well as isolated oligodendrocytes indicates ongoing myelin gene expression in recombined oligodendrocytes. The expression of genes associated with cholesterol synthesis are not reduced in isolated oligodendrocytes including the rate limiting enzyme for cholesterol synthesis (Hmgcr) and the enzyme responsible for the first committed step of cholesterol synthesis (Fdft1). On the tissue level, some of the analyzed cholesterol synthesis genes are reduced likely because of a reduction of cholesterol synthesis in other cell types such as neurons, microglia or astrocytes. Astrocytes are believed to be main producers of cholesterol in the brain (Pfrieger and Ungerer, 2011) and could potentially reduce their de novo cholesterol synthesis in response to recycling of myelin membrane under demyelinating conditions. While a slight reduction in cholesterol lipid abundance is observed in the optic nerve $8 \mathrm{~W}$ pti. This minor decrease is probably reflecting the loss of the compact myelin compartment and not a reduction in cholesterol synthesis. However, this evidence for continuous myelin protein and lipid synthesis by recombined oligodendrocytes supports the idea that the emerging tubules represent newly integrated myelin membranes and not a phenotype of local removal of aged MBP.

Since the shiverer-like membrane tubules appear predominantly at the inner tongue and the juxtaparanode we propose that these are metabolically active sites within the myelin sheath where myelin turnover takes place. Myelin membrane integration during development also takes place at the inner tongue (Snaidero et al., 2014) and membrane turnover has been hypothesized to happen at the paranode (Arancibia-Carcamo and Attwell, 2014). It is therefore conceivable that myelin integration during turnover takes place at the same sites as during development.

We propose that non-compact membranes observed at the inner tongue and the juxtaparanode are newly formed myelin membranes that in the absence of MBP are not able to compact and become part of the myelin sheath. Unequivocal direct evidence for the local 
integration of new myelin proteins at these sites into the adult myelin sheath could be obtained by pulse chase isotopic labeling followed by Nanoscale Secondary Ion Mass Spectrometry (NanoSIMS). Such experiments are currently ongoing.

\subsection{MBP loss and the half-life on an internode}

Turnover also implies removal of aged material. In our model the MBP which was synthetized and integrated into the myelin sheath during development is slowly lost from the system. To investigate whether MBP is diluted out of the myelin sheath we analyzed its lateral mobility within compact myelin. The lack of MBP mobility was determined by biochemical as well as immunoelectron microscopic evidence. We showed that in the iKO the mass of the isolated myelin fraction from brain lysate was reduced but contained wildtype levels of MBP. Accordingly, the labeling density of MBP on thin cryosections of iKO animals was the same on compact myelin compared to controls, but the total area of compact myelin was reduced. This evidence confirms that the compact myelin compartment is strictly defined by the presence of MBP (Kimura et al., 1989; Readhead and Hood, 1990). Furthermore, this result implies that due to the lack of lateral mobility MBP is not removed by dilution out of the myelin sheath. However, in contrast to MBP, myelin lipids have a high lateral mobility (Ando et al., 2003).

How is MBP then removed and replaced within compact myelin? Possibilities include increased levels of calcium influx leading to conformational changes of MBP and local opening of myelinic channels (Weil et al., 2016) followed by calcium dependent proteolysis by cathepsin D, as suggested by in vitro experiments (Banik et al., 1985). Another proposed mechanism involves citrullination of MBP by the myelin localized peptidyl arginine deiminase 2 (PADI2) which induces a reduction in MBP charge and conformational changes resulting in detaching from membrane, local decompaction and subsequent recycling (Moscarello et al., 2007; Wood et al., 2008). It is possible that these mechanisms of selective MBP removal are involved in the myelin turnover happening at the edges of compact myelin which are facing the non-compact myelin compartment. These questions remain to be addressed.

In our model we see direct consequences of MBP depletion in the form emergence of myelin tubules while at the same time physiological processes of myelin removal are continuing to take place. The emergence of non-compact myelin membrane is a direct result of lacking MBP and correlates with progressive loss of myelinated profiles in different CNS fiber tracts. Decreased density of myelinated axons in the optic nerve, caudal corpus callosum and the corticospinal tract of the spinal cord show that the loss of compact myelin takes place in all analyzed parts of the CNS. The kinetics of myelin loss as measured by the density of myelinated axons per $\mu \mathrm{m}^{2}$ was not uniform in all these tracts. For example, the density of myelinated axons reached a minimum at $26 \mathrm{~W}$ pti in the optic nerve compared to $52 \mathrm{~W}$ pti in the spinal cord. This might be due to differences in internodal length between CNS fiber tracts (Hildebrand et al., 1993; Bechler et al., 2015). Assuming that long internodes in the spinal cord (Friede et al., 1981; Bechler et al., 2015) are lost with a comparable speed than shorter internodes in the optic nerve, a decrease in myelinated profiles could appear later or be less pronounced in the spinal cord.

Quantification of myelinated and unmyelinated axons as fractions of total axon number in the optic nerve revealed a progressive decrease in myelinated axons and a corresponding increase in unmyelinated axon in the absence of axonal degeneration. To better understand 
the loss of myelinated profiles we performed 3D FIB-SEM analysis and found that many axons had reduced myelin coverage after ablation of MBP synthesis. Although the imaged internodes were mostly not completely contained in the volume, we obtained direct evidence for shortening of internodes. The 3D visualization revealed non-compact myelin membranes as well as detachment of paranodal loops at the edge of these internodes suggesting shortening by retraction from the node of Ranvier (Figure 4-1).

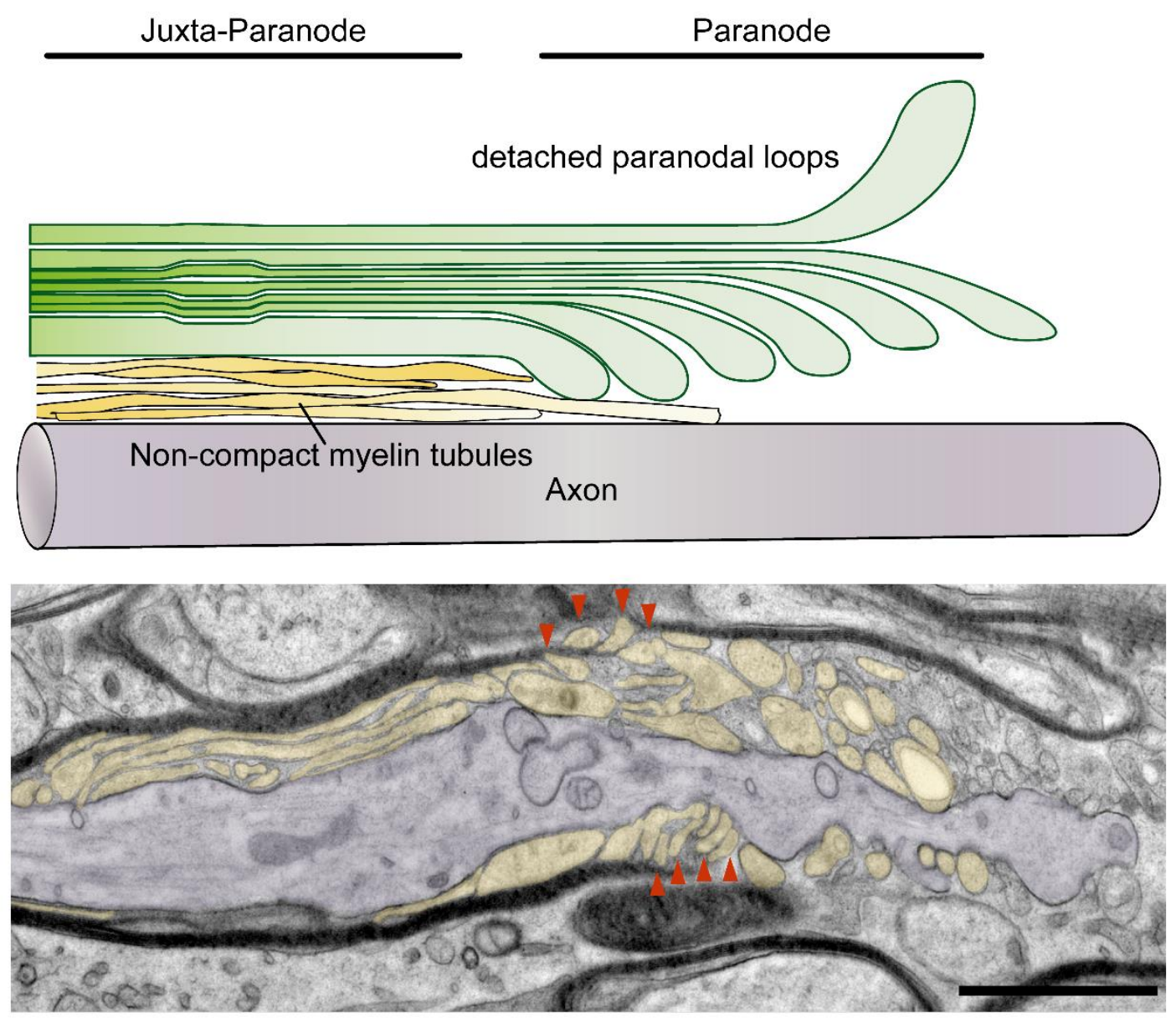

Figure 4-1 Indications for internodal shortening including paranode retraction

Presence of non-compact membrane tubules (orange) and detachment of paranodal loops (red arrow heads) from the axon (purple). HPF, Transmission electron micrograph of longitudinal optic nerve $26 \mathrm{~W}$ pti, scale bar $1 \mu \mathrm{m}$.

The comparison of reduced myelin coverage determined by 3D analysis and the reduced number of myelinated profiles on cross-sections yielded similar results. Therefore, the counting of myelinated profiles on cross-sections provides a valid estimate to quantify internodal shortening. Using the density of myelinated axons on a cross section of the optic nerve, we estimated that it takes 19-21 weeks (133-147 days) until 50\% of the myelin profiles were lost. This also corresponds to the time it takes to shorten an internode by half, thereby describing the half-life of an internode in our model in the optic nerve. We found that the myelin sheath is a highly stable structure with a half-life of 4 to 5 months, which we have determined by preventing the renewal of compact myelin and measuring its decay. With this genetic tool we could observe the half-life of a complete structure which can deviate from the half-life of its components. 
The stability of myelin has been addressed in several studies that quantified the half-life of individual myelin proteins in the brain (Sabri et al., 1974; Savas et al., 2012; Toyama et al., 2013; Fornasiero et al., 2018). The components of the myelin sheath were frequently found belonging to the most long-lived proteins beside histones and nuclear pore in brain (Toyama et al., 2013; Fornasiero et al., 2018). The most recent publication by Fornaserio and colleagues used sophisticated reutilization models to calculate precise half-lives of myelin proteins from different brain regions. MBP has been found to have a half-life between 82 days in cortex and 70 days in a special long pulse experiment designed for proteins with a long half-life. Also other myelin proteins have very long half-lives like PLP (80 days), tetraspanin-2 (90 days), MOG (164 days) and claudin-11 (139 days) (Fornasiero et al., 2018). In contrast CNP has a half-life of around 53 days being a rather short-lived myelin protein and the only non-compact myelin protein the authors selected for comparison. The average half-life of the compact myelin proteins listed here is about 109 days reflecting the high stability of the myelin sheath. We calculated an MBP half-life of approximately 77 days which is in close agreement with the previously published 70-82 days (Fornasiero et al., 2018) A study by Toyama and colleagues found that after a chase of 6 months 20\% of initially labeled MBP was still present (Toyama et al., 2013). This is comparable to the 25\% remaining MBP that was found in our model 26 weeks post tamoxifen induction. The remaining difference might be explained by presence of MBP from non-recombined and newly differentiated oligodendrocytes.

However, looking at the structure of the myelin sheath we have found that the half-lives for PLP, MBP and tetraspanin-2 in the brain are shorter than our estimation of 133-147 days as half-life of the complete myelin sheath in the optic nerve. An explanation could be that $25 \%$ expression of $M b p$ mRNA is sufficient to form compact myelin (Readhead et al., 1987), also heterozygous shiverer mice that have $50-70 \%$ of MBP protein compared to control have functional and normal appearing compact myelin (Poggi et al., 2016). Even with decreased levels of MBP in compact myelin, for example by MBP removal due to limited lateral mobility, the myelin membrane could still be compact and functional. Additionally, the observed thinning of compact myelin could also contribute to MBP loss without necessarily affecting internodal length.

The observed changes in internodal length in our model could partially reflect a physiological mechanism of myelin remodeling. Life imaging experiments on zebrafish have shown sheath elongation and retraction as physiological process during development (Czopka et al., 2013) and chronic imaging of myelin internode in the mouse cortex shows changes in internode length (Hill et al., 2018). Growth and shrinkage of internodes albeit without the observed loss of paranodal adhesion to the axon in this model could be a way for mature oligodendrocytes to remodel their myelin sheath.

In conclusion, we find that continuous synthesis of MBP is required to maintain myelin internodes as well as nodes of Ranvier. Absence of MBP results in shortening of internodes and loss of node structure by progressive demyelination. Here, we identify the paranodal and juxta-paranodal region, first affected by loss of MBP synthesis, as an important site for myelin rearrangements and renewal. 


\subsection{Myelin removal by oligodendrocytes}

As described above the decrease in MBP abundance in brain lysates suggests a faster loss of MBP than expected from internode shortening alone. The increase in corrected g-ratio in the optic nerve at $16 \mathrm{~W}$ and $26 \mathrm{~W}$ pti indicates also a reduction in myelin thickness. This loss of compact myelin by shortening of internodes together with thinning of compact myelin might reflect the consequences of the observed loss of MBP protein abundance more closely.

Since MBP-dilution was shown to be less plausible as a mechanism of MBP turnover due to lacking lateral mobility, more likely compact myelin as a whole undergoes removal. In principle two different routes of removal could be followed. Intracellular removal by the oligodendrocyte or extracellular removal by other cells like astrocytes and microglia. Microglia (Safaiyan et al., 2016) as well as astrocytes (Mills et al., 2015; Ponath et al., 2017) are implicated in removal of myelin during aging or demyelination. Especially astrocytes that can form direct contact at the node of Ranvier (Butt et al., 1994) and might be involved in active uptake of myelin. Accordingly, we have observed microglial uptake of myelin debris as well as myelin protrusions into astrocyte processes in our 3D data.

In addition to these events, the 3D FIB-SEM data of control and iKO mice revealed the presence of myelin membrane structures at the inner tongue and outside of compact myelin. These compact myelin spheres have previously been described as myelin spheroids in aged brains of rhesus monkeys (Peters and Sethares, 2002) or as Marchi Bodies found in normal white matter (Hildebrand and Aldskogius, 1976) often localized close to the paranode (Corneliuson et al., 1987). Compact myelin spheres at the inner tongue called myelinosomes were also recently described as an early stage of myelin damage in EAE lesions where they were actively taken up by macrophages (Romanelli et al., 2016). A recent life imaging study found increased numbers of myelin spheres during aging and could follow the formation and degradation of these structures showing that generation and removal can take several weeks (Hill et al., 2018).

We found compact myelin spheres predominantly at the inner tongue of control as well as iKO animals $16 \mathrm{~W}$ and $26 \mathrm{~W}$ pti. Using FIB-SEM acquisition with a high XY (5 nm pixel size) and $\mathrm{Z}$ (10 $\mathrm{nm}$ step size) resolution we could identify also smaller myelin spheres as infrequent evens of control animals. This finding suggests that myelin spheres are present not only during demyelination but also under normal conditions. Since these myelin spheres at the inner tongue are rare, they were probably overlooked or mistaken as an artefact by conventional electron microscopy on thin sections. Only 3D electron microscopy data analysis revealed that these spheres are connected to the myelin sheath. Myelin spheres were present independent of the direct presence of microglia or astrocytes contacting the myelin sheath and significantly increased in number in iKO mice. Although the local thinning of the myelin sheath was not found to be directly associated with the presence of myelin spheres at the inner tongue I think that they represent an oligodendrocyte-intrinsic removal mechanism of aged myelin. Since these structures are also found in normal white matter it is likely that they resemble a cell-intrinsic mechanism independent of demyelination. This is supported by knock-out of the autophagosomal marker Atg7 (Arakawa et al., 2017) in oligodendrocytes. Ablation of the autophagosomal machinery in mice results in increased myelin thickness at the age of 16 months suggesting an involvement of autophagy on myelin maintenance (Aber, 2018). 


\subsection{Systemic response to ablation of MBP synthesis}

To investigate the systemic response of MBP loss on the myelinating system we performed proteome analysis of whole optic nerve at early and late time points and from shiverer mice at an age of 10 weeks for comparison with a model completely lacking MBP. Time points $8 \mathrm{~W}$ and $16 \mathrm{~W}$ pti were included to observe the systemic response to ablation of MBP synthesis under low levels of inflammation in a mostly unperturbed system. The $40 \mathrm{~W}$ pti time point was included to investigate a late inflammatory state of ongoing compact myelin loss at the peak of symptoms.

Changes in protein abundance in optic nerve $8 \mathrm{~W}$ and $16 \mathrm{~W}$ pti included reduced levels of myelin proteins (MBP, PLP, CD9, Opalin) which probably reflected the loss of compact myelin compartment by internode shortening and reduction in myelin thickness. On a tissue level, lipid synthesis proteins such as CGT (only identified in $8 \mathrm{~W}$ pti) involved in galactocerebrosides synthesis and FPPS (FDPS) (identified in all time points) required for cholesterol synthesis were reduced in abundance. In contrast, mRNA expression data of isolated oligodendrocytes showed no overall changes in cholesterol synthesis genes including FPPS (FDPS). A similar effect was observed in a transcriptome analysis of shiverer where cholesterol synthesis genes were reduced in shiverer brain but unchanged in isolated and cultured shiverer oligodendrocytes (Aaker et al., 2016). Since FPPS (FDPS) is also expressed in astrocytes (Zhang et al., 2014) we believe that reduced levels can be attributed to changes in astrocytic cholesterol synthesis due to uptake of cholesterol rich myelin debris.

Another class of proteins reduced in abundance are tetraspanins and cell adhesion molecules (TSN2, Protein 4.1, CD81, NCAM1). Since these are also major myelin proteins (Jahn et al., 2009) their abundance could be decreased by loss of compact myelin. CD9 and CD81 are also involved in Schwann cell to axon interaction (Chernousov et al., 2013) and it could be possible that the same proteins are involved here in the loss of oligodendrocyteto-axon adhesion during internode retraction.

Proteins increased in abundance in the optic nerve $8 \mathrm{~W}$ and $16 \mathrm{~W}$ pti were associated with lipid transport (APOE, APOD) and the cytoskeleton (ARF2/3, fascin and tubulin). In the PNS Apod mRNA is upregulated up to 500 fold during de- and remyelination (Boyles et al., 1990) and was suggested to be involved in clearance and recycling of myelin debris (Muffat and Walker, 2010; García-Mateo et al., 2018). Increased abundance of cytoskeletal proteins could be explained by emergence of non-compact myelin membrane tubules. The observed structures, in comparison to compact myelin, contained microtubules as seen on the TEM images. Another possible explanation is an increased density of axonal microtubules as seen in shiverer mice (Kirkpatrick et al., 2001). Due to high levels of protein homology it was not possible to resolve individual tubulin species. We therefore cannot distinguish between isoforms specific for oligodendrocytes (beta IV tubulin) and axons (beta III tubulin). In addition to changes observed at $8 \mathrm{~W}$ and $16 \mathrm{~W}$ the $40 \mathrm{~W}$ pti time point showed a significant increase in microglial markers, degradative enzymes (HEXB, CATD, CATZ, CATS) (Zhang et al., 2014) and components of the complement system (C1QC, C1CB). This indicated immune system activation, inflammation, microgliosis (HEXB) and astrogliosis (AQP4).

Optic nerves from shiverer had a strong reduction in myelin proteins (MOG, PLP (MPYR), CLD11, MAG) as well as cytoskeletal proteins (SEPT2/4, ANLN) likely reflecting the near complete absence of compact myelin and therefore reduced levels of all proteins associated to compact myelin, as expected. Proteins strongly increased in abundance included 
ribosomal proteins (60S, RPL4/7a/5) indicating an increased protein translation that could be caused by the ongoing effort of oligodendrocytes to form compact myelin and insulate axons. Another class of proteins with increased abundance are associated with energy metabolism (PGAM2, KCRB, AT1A1). This argues for increased energy requirements in the absence of compact myelin (Andrews et al., 2006)

Complementary to the analysis of the top most affected 30 proteins, pathway enrichment analysis of all significantly increased proteins in $8 \mathrm{~W}, 40 \mathrm{~W}$ pti and shiverer was performed. Enrichment of pathways involved in energy metabolism (TCA cycle, glycolysis) in all groups suggested an increased energy requirement after the loss of compact myelin. This is supported by previously reported increase in mitochondrial size and speed of transport for axonal mitochondria after demyelination (Kiryu-Seo et al., 2010). Other enriched pathways include the proteasome pathway and phagosomes at $40 \mathrm{~W}$ pti probably caused by increased myelin debris clearing either by microglia or, as discussed, also by oligodendrocytes.

To summarize, proteome analysis revealed that myelin proteins and cell adhesion proteins are reduced while the levels of cytoskeletal proteins and lipid transport proteins are increased. As expected, the time points $8 \mathrm{~W}$ and $16 \mathrm{~W}$ pti show low levels of inflammation, while at the time point $40 \mathrm{~W}$ pti signs of stronger astrogliosis and microgliosis are detected. All observed time points including shiverer show an increase in proteins associated to energy metabolism which could reflect the loss of electrical insulation by compact myelin and the corresponding higher energy requirements for action potential conduction.

We performed lipid analysis since we expected major changes in lipid composition of noncompacted myelin membrane. This was motivated by increased levels of phosphatidylserine and cholesterol esters and decreased levels of sphingomyelin, phosphatidylcholine and cerebroside sulfates found in white matter lesions of an EAE model in marmosets (Ohler et al., 2004) Ohler and colleagues hypothesized that these changes lead to increased membrane fluidity and curvature resulting in reduced MBP adhesion and destabilization of the myelin sheath (Ohler et al., 2004).

We investigate changes in lipid composition after emergence of non-compact membrane tubules by mass spectrometry analysis in optic nerve and corpus callosum. Focusing on the optic nerve $16 \mathrm{~W}$ pti we found significant increase in phosphatidylinositol (PI) levels. Changes in PI signaling have been previously been reported in an pathway enrichment analysis in transcriptome of shiverer brain (Aaker et al., 2016). The phospholipid phosphatidylinositol 4,5-bisphosphate facilitates stable association between MBP and cellular membranes (Nawaz et al., 2013) and phosphorylation of phosphoinositide regulates the ability of MBP to bind actin filaments to the membrane (Boggs et al., 2012). PI levels are also known to have immunoregulatory effects by limiting proinflammatory cytokines and inhibiting the inflammatory $\mathrm{T}$-cell response in a model of chronic inflammation (van Dieren et al., 2011). This suggests that a beginning inflammatory gliosis could also be responsible for the increased levels of PI. The performed analysis did not allow distinction between PIP2 and other PIP forms. I speculate that increased levels of PI could be a consequence of either the emergence of non-compact membrane tubules or an inflammatory response. Additional analysis of PI derivates and lipid composition is required to draw further conclusions.

The lipids phosphatidylglycerol (PG) and lysophosphatidyl choline (LPC) showed a tendency for increased abundance without passing the significance threshold in optic nerve $16 \mathrm{~W}$ pti but did so in other analyzed time points. Elevated levels of endogenous LPC occur after LPC induced demyelination (Plemel et al., 2018). Based on cell culture experiments the 
authors hypothesized that LPC has a lipid buffer function to ameliorate toxic effects of demyelination (Plemel et al., 2018). Elevated levels of PG are associated with inhibition of inflammation in a macrophage cell culture model (Chen et al., 2018). Based on these results we hypothesize that elevated levels of LPC and PG could be due to a beginning inflammatory response to the ongoing loss of compact myelin after ablation of MBP synthesis. We did not find comparable changes in lipid composition as reported for whiter matter lesions in EAE (Ohler et al., 2004). This further suggests that our model of genetic demyelination is not comparable to the immune mediated breakdown of myelin in the EAE mode.

\subsection{Late remyelination after MBP ablation}

The observed motor phenotype including tremor and motor impairment as assessed by the performed "box test" emerges at 16 to $26 \mathrm{~W}$ pti. At $26 \mathrm{~W}$ pti the density of myelinated axons is decreased in different myelinating tracts including the spinal cord, but not in the PNS indicating CNS pathology. The tremor phenotype peaks between $30 \mathrm{~W}$ and $40 \mathrm{~W}$ pti and thereafter decreases in severity. Also, the loss of weight decreases in speed, especially in female mice. This partial amelioration suggested a compensatory mechanism.

By analysis of protein expression and morphological assessment of remyelination in the optic nerve we found indications of remyelination by newly differentiated OPC which occur alongside with recombined oligodendrocytes. This was possible since the used PLP-Cre ${ }^{\text {ERT2 }}$ driver line allows for recombination of mature oligodendrocytes without affecting OPCs (Leone et al., 2003).

We conclude that remyelination has taken place after ablation of MBP synthesis in adult oligodendrocytes. It is however puzzling why remyelination occurred at such a late time point. Other models of adult demyelination such as cuprizone (Torkildsen et al., 2008; Pfeifenbring et al., 2015) or LPC (Woodruff and Franklin, 1999; Ghasemlou et al., 2007) are showing signs of remyelination after days to weeks. Major differences between these models and the model used here is the lack of widespread oligodendrocyte death, relatively low levels of inflammation and the ongoing presence of myelin debris in form of noncompact myelin membrane. In multiple sclerosis, the presence of OPCs in a demyelinating lesion is not sufficient to induce remyelination, if the environment is inhibitory to OPC maturation (Chang et al., 2002). Known inhibitors for OPC differentiation and negative regulators of myelination include the presence of PSA-NCAM on axons (Charles et al., 2002) the astrocyte generated glycosaminoglycan hyaluron (Back et al., 2005) LINGO-1 as expressed on oligodendrocytes (Mi et al., 2005, 2007) and the presence of myelin debris(Kotter et al., 2006).

The survival of recombined oligodendrocytes, which might express inhibitory factors such as LINGO-1 (Mi et al., 2009), could prevent maturation of new oligodendrocytes. However, in our model the number of CAII+ cells is still increased in the fimbria even at $52 \mathrm{~W}$ pti due to the lack of widespread oligodendrocyte death even at that late time point when remyelination has occurred in the brain. 


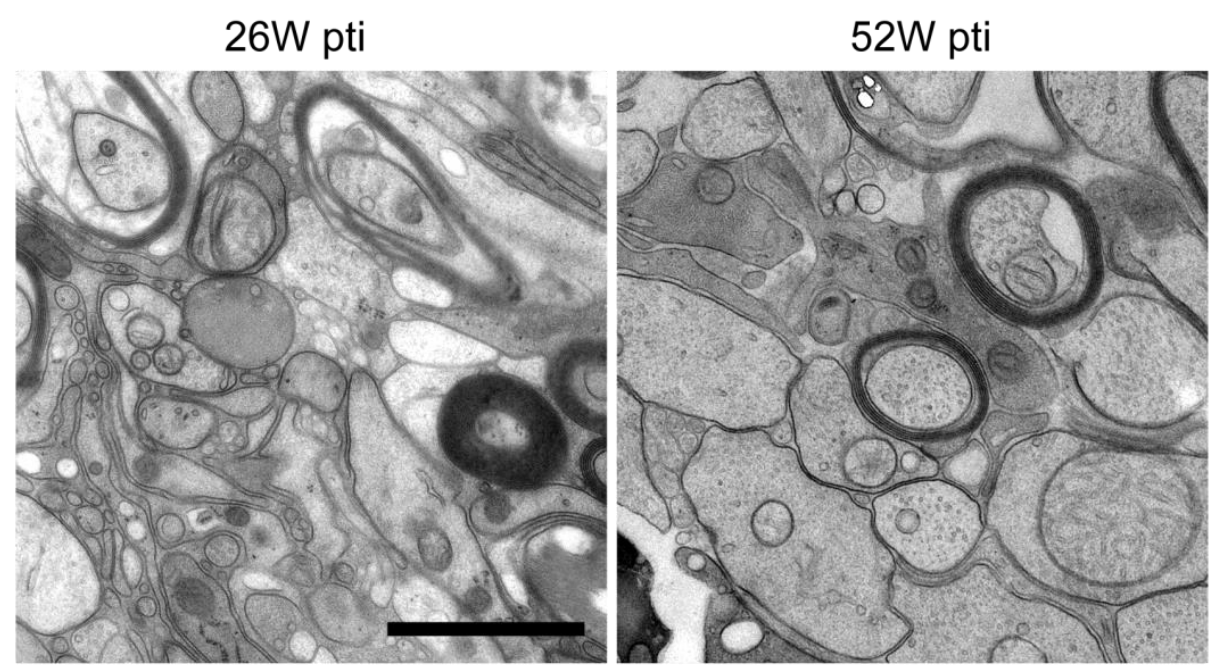

Figure 4-2 Presence of non-compact myelin tubules and myelin debris may inhibit remyelination

Increased presence of non-compact myelin tubules and myelin debris at $26 \mathrm{~W}$ pti might inhibit maturation of OPC and remyelination. iKO optic nerve, HPF, scale bar $1 \mu \mathrm{m}$.

Another factor known to inhibit remyelination is the presence of myelin debris. Injection of myelin after a demyelinating event arrests differentiation of OPCs at a premyelinating state (Kotter et al., 2006). It is suggested that phagocytic clearance of myelin debris is affecting remyelination (Kotter et al., 2006). Within the first $26 \mathrm{~W}$ pti we have observed minor levels of microgliosis in brain. At $52 \mathrm{~W}$ pti we saw a striking increase in MAC- $3^{+}$cells in the fimbria which is indicative for phagocytic activity of microglia probably mediating the uptake of myelin debris. Electron micrographs of optic nerve show that at $26 \mathrm{~W}$ at the beginning of remyelination high levels of non-compact myelin membrane are found. In contrast, the levels of non-compact myelin membrane (Figure 4-2) are reduced at $52 \mathrm{~W}$ pti when distinct remyelination has taken place. We therefore speculate that the presence of non-compact myelin tubules as well as myelin debris might inhibit remyelination and that this inhibition is finally resolved after microglial activation and clearance of myelin debris. 


\subsection{Summary}

Myelin turnover in the adult animal is a crucial process to allow stability and maintenance of the myelin sheath once it has been wrapped around the axon. The aim of this thesis was to investigate myelin maintenance and turnover by ablating synthesis of MBP in mature myelinating oligodendrocytes after completion of developmental myelination. For this, the inducible $M b p^{f l o x}$ fllox*PlpCreERT2 mouse model was introduced and characterized. Due to its low levels of inflammation, the survival of oligodendrocytes after knock-out induction and ongoing synthesis of myelin transcripts it fulfills the requirements needed to study myelin turnover in the adult mouse. Membrane tubules formed in the absence of MBP and emerged at the inner tongue primarily in the juxtaparanodal region, hence this being the likely site of myelin membrane insertion and turnover within the myelin internode. Ongoing synthesis of MBP was shown to be essential for maintenance of compact myelin. In the absence of MBP de novo synthesis the levels of MBP and other compact myelin proteins, the length of myelin internodes and myelin thickness decreased progressively. Approximate half replacement times of myelin internodes in the optic nerve of 19-21 weeks after ablation of MBP synthesis support the assumption that myelin and its components are stable and long lived but require replacement within weeks to months. Identification of compact myelin spheres at the inner tongue suggests an additional oligodendrocyte-specific mechanism for removal of aged myelin besides microglial or astrocytic uptake. This supports the hypothesis that myelin turnover is mediated by extracellular as well as intracellular mechanisms. The occurrence of remyelination in brain and optic nerve provided evidence that mainly adult oligodendrocytes were targeted by tamoxifen-induced recombination while OPCs were not. The late onset of remyelination after beginning of the shortening of internodes suggests that presence of myelin debris as well as surviving oligodendrocytes inhibit remyelination.

This thesis improves the understanding of myelin maintenance and turnover in the adult mouse CNS by providing a new mouse model, which made it possible to localize sites of myelin membrane integration and removal within the myelin internode by advanced electron microscopic techniques this thesis improves the understanding of myelin maintenance and turnover in the adult mouse CNS. The Determination of the mechanisms of myelin turnover will increase the understanding of physiological maintenance processes as well as pathological changes in diseases. 


\section{Bibliography}

Aaker JD, Elbaz B, Wu Y, Looney TJ, Zhang L, Lahn BT, Popko B (2016) Transcriptional Fingerprint of Hypomyelination in Zfp191 null and Shiverer ( Mbp shi ) Mice. ASN Neuro 8:175909141667074 Available at:

http://www.ncbi.nlm.nih.gov/pubmed/27683878 [Accessed April 6, 2019].

Aber E (2018) Myelin is remodeled cell-autonomously by oligodendroglial macroautophagy. Available at:

https://academiccommons.columbia.edu/doi/10.7916/D8V99M16.

Aggarwal S, Yurlova L, Simons M (2011a) Central nervous system myelin: structure, synthesis and assembly. Trends Cell Biol 21:585-593 Available at: http://www.ncbi.nlm.nih.gov/pubmed/21763137 [Accessed February 27, 2019].

Aggarwal S, Yurlova L, Snaidero N, Reetz C, Frey S, Zimmermann J, Pähler G, Janshoff A, Friedrichs J, Müller DJ, Goebel C, Simons M (2011b) A Size Barrier Limits Protein Diffusion at the Cell Surface to Generate Lipid-Rich Myelin-Membrane Sheets. Dev Cell 21:445-456 Available at: https://www.sciencedirect.com/science/article/pii/S1534580711003133 [Accessed March 4, 2019].

Akiyama K, Ichinose S, Omori A, Sakurai Y, Asou H (2002) Study of expression of myelin basic proteins (MBPs) in developing rat brain using a novel antibody reacting with four major isoforms of MBP. J Neurosci Res 68:19-28 Available at: http://doi.wiley.com/10.1002/jnr.10188 [Accessed March 8, 2019].

Allen NJ, Barres BA (2009) Neuroscience: Glia - more than just brain glue. Nature 457:675-677 Available at: http://dx.doi.org/10.1038/457675a [Accessed February $27,2019]$.

Allen NJ, Eroglu C (2017) Cell Biology of Astrocyte-Synapse Interactions. Neuron 96:697708 Available at: https://linkinghub.elsevier.com/retrieve/pii/S089662731730925X.

Allinquant B, Staugaitis SM, D'Urso D, Colman DR (1991) The ectopic expression of myelin basic protein isoforms in Shiverer oligodendrocytes: implications for myelinogenesis. J Cell Biol 113:393-403 Available at: http://www.ncbi.nlm.nih.gov/pubmed/1707056 [Accessed March 5, 2019].

Almeida A, Almeida J, Bolaños JP, Moncada S (2001) Different responses of astrocytes and neurons to nitric oxide: the role of glycolytically generated ATP in astrocyte protection. Proc Natl Acad Sci U S A 98:15294-15299 Available at: http://www.pnas.org/cgi/doi/10.1073/pnas.261560998 [Accessed February 27, 2019].

Ambrozkiewicz MC et al. (2018) Polarity Acquisition in Cortical Neurons Is Driven by Synergistic Action of Sox9-Regulated Wwp1 and Wwp2 E3 Ubiquitin Ligases and Intronic miR-140. Neuron 100:1097-1115.e15 Available at: http://www.ncbi.nlm.nih.gov/pubmed/30392800 [Accessed March 17, 2019].

Ando S, Tanaka Y, Toyoda Y, Kon K (2003) Turnover of myelin lipids in aging brain. Neurochem Res 28:5-13 Available at: http://www.ncbi.nlm.nih.gov/pubmed/12587659 [Accessed February 27, 2019].

Andrews H, White K, Thomson C, Edgar J, Bates D, Griffiths I, Turnbull D, Nichols P (2006) Increased axonal mitochondrial activity as an adaptation to myelin deficiency in the 
Shiverer mouse. J Neurosci Res 83:1533-1539 Available at: http://doi.wiley.com/10.1002/jnr.20842 [Accessed March 5, 2019].

Arakawa S, Honda S, Yamaguchi H, Shimizu S (2017) Molecular mechanisms and physiological roles of Atg5/Atg7-independent alternative autophagy. Proc Jpn Acad Ser B Phys Biol Sci 93:378-385 Available at: http://www.ncbi.nlm.nih.gov/pubmed/28603209.

Arancibia-Carcamo IL, Attwell D (2014) The node of Ranvier in CNS pathology. Acta Neuropathol 128:161-175 Available at: http://link.springer.com/10.1007/s00401014-1305-z [Accessed April 5, 2019].

Arandjelovic S, Ravichandran KS (2015) Phagocytosis of apoptotic cells in homeostasis. Nat Immunol 16:907-917 Available at: http://www.ncbi.nlm.nih.gov/pubmed/26287597 [Accessed February 27, 2019].

Arguedas D, Batchelor J (2009) 18q Deletion syndrome: A neuropsychological case study. Neurocase 15:101-109 Available at: http://www.tandfonline.com/doi/abs/10.1080/13554790802631928 [Accessed March 5, 2019].

Arroyo EJ, Xu T, Poliak S, Watson M, Peles E, Scherer SS (2001) Internodal specializations of myelinated axons in the central nervous system. Cell Tissue Res 305:53-66 Available at: http://link.springer.com/10.1007/s004410100403 [Accessed March 3, 2019].

Back SA, Tuohy TMF, Chen H, Wallingford N, Craig A, Struve J, Luo NL, Banine F, Liu Y, Chang A, Trapp BD, Bebo, BF, Rao MS, Sherman LS (2005) Hyaluronan accumulates in demyelinated lesions and inhibits oligodendrocyte progenitor maturation. Nat Med 11:966-972 Available at: http://www.ncbi.nlm.nih.gov/pubmed/16086023 [Accessed April 4, 2019].

Bamm V V, De Avila M, Smith GST, Ahmed MAM, Harauz G (2011) Structured functional domains of myelin basic protein: cross talk between actin polymerization and $\mathrm{Ca}(2+)$-dependent calmodulin interaction. Biophys J 101:1248-1256 Available at: http://www.pubmedcentral.nih.gov/articlerender.fcgi?artid=3164172\&tool=pmcent rez\&rendertype=abstract.

Banik NL, McAlhaney WW, Hogan EL (1985) Calcium-stimulated proteolysis in myelin: evidence for a Ca2+-activated neutral proteinase associated with purified myelin of rat CNS. J Neurochem 45:581-588 Available at:

http://www.ncbi.nlm.nih.gov/pubmed/2409235 [Accessed April 4, 2019].

Barbarese E, Braun PE, Carson JH (1977) Identification of prelarge and presmall basic proteins in mouse myelin and their structural relationship to large and small basic proteins. Proc Natl Acad Sci U S A 74:3360-3364 Available at: http://www.ncbi.nlm.nih.gov/pubmed/71732 [Accessed March 4, 2019].

Battefeld A, Tran BT, Gavrilis J, Cooper EC, Kole MHP (2014) Heteromeric Kv7.2/7.3 Channels Differentially Regulate Action Potential Initiation and Conduction in Neocortical Myelinated Axons. J Neurosci 34:3719-3732 Available at: http://www.ncbi.nlm.nih.gov/pubmed/24599470 [Accessed March 3, 2019].

Baumann N, Pham-Dinh D (2001) Biology of oligodendrocyte and myelin in the mammalian central nervous system. Physiol Rev 81:871-927 Available at: http://www.physiology.org/doi/10.1152/physrev.2001.81.2.871 [Accessed February 27, 2019]. 
Bechler ME, Byrne L, Ffrench-Constant C (2015) CNS Myelin Sheath Lengths Are an Intrinsic Property of Oligodendrocytes. Curr Biol 25:2411-2416 Available at: http://www.ncbi.nlm.nih.gov/pubmed/26320951 [Accessed April 5, 2019].

Belevich I, Joensuu M, Kumar D, Vihinen H, Jokitalo E (2016) Microscopy Image Browser: A Platform for Segmentation and Analysis of Multidimensional Datasets. PLOS Biol 14:e1002340 Available at: http://dx.plos.org/10.1371/journal.pbio.1002340 [Accessed March 17, 2019].

Beniac DR, Wood DD, Palaniyar N, Ottensmeyer FP, Moscarello MA, Harauz G (2000) Cryoelectron Microscopy of Protein-Lipid Complexes of Human Myelin Basic Protein Charge Isomers Differing in Degree of Citrullination. J Struct Biol 129:80-95 Available at: http://www.ncbi.nlm.nih.gov/pubmed/10675299 [Accessed March 5, 2019].

Benveniste EN (1997) Role of macrophages/microglia in multiple sclerosis and experimental allergic encephalomyelitis. J Mol Med (Berl) 75:165-173 Available at: http://www.ncbi.nlm.nih.gov/pubmed/9106073.

Berger T, Reindl M (2000) Immunopathogenic and clinical relevance of antibodies against myelin oligodendrocyte glycoprotein (MOG) in Multiple Sclerosis. In: Advances in Research on Neurodegeneration, pp 351-360. Vienna: Springer Vienna. Available at: http://www.springerlink.com/index/10.1007/978-3-7091-6301-6_25 [Accessed March 6, 2019].

Berghs S, Aggujaro D, Dirkx R, Maksimova E, Stabach P, Hermel JM, Zhang JP, Philbrick W, Slepnev V, Ort T, Solimena M, Solimena M (2000) betaIV spectrin, a new spectrin localized at axon initial segments and nodes of ranvier in the central and peripheral nervous system. J Cell Biol 151:985-1002 Available at: http://www.ncbi.nlm.nih.gov/pubmed/11086001 [Accessed March 3, 2019].

Biffiger K, Bartsch S, Montag D, Aguzzi A, Schachner M, Bartsch U (2000) Severe Hypomyelination of the Murine CNS in the Absence of Myelin-Associated Glycoprotein and Fyn Tyrosine Kinase. J Neurosci 20:7430-7437 Available at: http://www.ncbi.nlm.nih.gov/pubmed/11007902 [Accessed April 12, 2019].

Bird TD, Farrell DF, Sumi SM (1978) Brain lipid composition of the shiverer mouse: (genetic defect in myelin development). J Neurochem 31:387-391 Available at: http://doi.wiley.com/10.1111/j.1471-4159.1978.tb12479.x [Accessed March 5, 2019].

Black JA, Waxman SG (1988) The perinodal astrocyte. Glia 1:169-183 Available at: http://www.ncbi.nlm.nih.gov/pubmed/2976037 [Accessed March 3, 2019].

Blakemore WF (1974) Pattern of remyelination in the CNS. Nature 249:577-578 Available at: http://www.nature.com/articles/249577a0 [Accessed March 24, 2019].

Blum H, Beier H, Gross HJ (1987) Improved silver staining of plant proteins, RNA and DNA in polyacrylamide gels. Electrophoresis 8:93-99 Available at: http://doi.wiley.com/10.1002/elps.1150080203 [Accessed March 17, 2019].

Boggs JM, Rangaraj G, Dicko A (2012) Effect of phosphorylation of phosphatidylinositol on myelin basic protein-mediated binding of actin filaments to lipid bilayers in vitro. Biochim Biophys Acta - Biomembr 1818:2217-2227 Available at: https://www.sciencedirect.com/science/article/pii/S0005273612001289?via\%3Di hub\#f0005 [Accessed April 7, 2019].

Boggs JM, Wood DD, Moscarello MA, Papahadjopoulos D (1977) Lipid phase separation induced by a hydrophobic protein in phosphatidylserine--phosphatidylcholine 
vesicles. Biochemistry 16:2325-2329 Available at:

http://www.ncbi.nlm.nih.gov/pubmed/577185 [Accessed March 4, 2019].

Boiko T, Rasband MN, Levinson SR, Caldwell JH, Mandel G, Trimmer JS, Matthews G (2001) Compact myelin dictates the differential targeting of two sodium channel isoforms in the same axon. Neuron 30:91-104 Available at: http://www.ncbi.nlm.nih.gov/pubmed/11343647 [Accessed March 3, 2019].

Boullerne AI (2016) The history of myelin. Exp Neurol 283:431-445 Available at: http://www.ncbi.nlm.nih.gov/pubmed/27288241 [Accessed February 27, 2019].

Boyles JK, Notterpek LM, Anderson LJ (1990) Accumulation of apolipoproteins in the regenerating and remyelinating mammalian peripheral nerve. Identification of apolipoprotein D, apolipoprotein A-IV, apolipoprotein E, and apolipoprotein A-I. J Biol Chem 265:17805-17815 Available at: http://www.ncbi.nlm.nih.gov/pubmed/2120218 [Accessed April 6, 2019].

Brunner C, Lassmann H, Waehneldt T V., Matthieu J-M, Linington C (1989) Differential Ultrastructural Localization of Myelin Basic Protein, Myelin/Oligodendroglial Glycoprotein, and 2',3'-Cyclic Nucleotide 3'-Phosphodiesterase in the CNS of Adult Rats. J Neurochem 52:296-304 Available at: http://doi.wiley.com/10.1111/j.14714159.1989.tb10930.x [Accessed March 6, 2019].

Bu J, Banki A, Wu Q, Nishiyama A (2004) Increased NG2+ glial cell proliferation and oligodendrocyte generation in the hypomyelinating mutant shiverer. Glia 48:51-63 Available at: http://doi.wiley.com/10.1002/glia.20055 [Accessed March 5, 2019].

Butt A, Verkhratsky A (2013) Neuroglia: Definition, Classification, Evolution, Numbers, Development. In: Glial Physiology and Pathophysiology, pp 73-104. Chichester, UK: John Wiley \& Sons, Ltd. Available at: http://doi.wiley.com/10.1002/9781118402061.ch3 [Accessed March 22, 2019].

Butt AM, Duncan A, Berry M (1994) Astrocyte associations with nodes of Ranvier: ultrastructural analysis of HRP-filled astrocytes in the mouse optic nerve. J Neurocytol 23:486-499 Available at: http://www.ncbi.nlm.nih.gov/pubmed/7983475 [Accessed March 3, 2019].

Butt AM, Duncan A, Hornby MF, Kirvell SL, Hunter A, Levine JM, Berry M (1999) Cells expressing the NG2 antigen contact nodes of Ranvier in adult CNS white matter. Glia 26:84-91 Available at: http://www.ncbi.nlm.nih.gov/pubmed/10088675 [Accessed March 3, 2019].

Campagnoni AT, Pribyl TM, Campagnoni CW, Kampf K, Amur-Umarjee S, Landry CF, Handley VW, Newman SL, Garbay B, Kitamura K (1993) Structure and developmental regulation of Golli-mbp, a 105-kilobase gene that encompasses the myelin basic protein gene and is expressed in cells in the oligodendrocyte lineage in the brain. J Biol Chem 268:4930-4938 Available at:

http://www.ncbi.nlm.nih.gov/pubmed/7680345 [Accessed March 4, 2019].

Campbell GR, Mahad DJ (2014) Metabolic support of axons by oligodendrocytes: Implications for multiple sclerosis. Mult Scler Relat Disord 3:28-30 Available at: http://www.ncbi.nlm.nih.gov/pubmed/25877969 [Accessed February 27, 2019].

Cantoni C, Bollman B, Licastro D, Xie M, Mikesell R, Schmidt R, Yuede CM, Galimberti D, Olivecrona G, Klein RS, Cross AH, Otero K, Piccio L (2015) TREM2 regulates microglial cell activation in response to demyelination in vivo. Acta Neuropathol 129:429-447 Available at: http://www.ncbi.nlm.nih.gov/pubmed/25631124 [Accessed February $27,2019]$. 
Cantuti-Castelvetri L, Fitzner D, Bosch-Queralt M, Weil M-T, Su M, Sen P, Ruhwedel T, Mitkovski M, Trendelenburg G, Lütjohann D, Möbius W, Simons M (2018) Defective cholesterol clearance limits remyelination in the aged central nervous system. Science (80-) 359:684-688 Available at: http://www.ncbi.nlm.nih.gov/pubmed/29301957 [Accessed March 6, 2019].

Cao L, Goodin R, Wood D, Moscarello MA, Whitaker JN (1999) Rapid Release and Unusual Stability of Immunodominant Peptide 45-89 from Citrullinated Myelin Basic Protein †. Biochemistry 38:6157-6163 Available at: http://www.ncbi.nlm.nih.gov/pubmed/10320343 [Accessed March 5, 2019].

Caprariello A V, Rogers JA, Morgan ML, Hoghooghi V, Plemel JR, Koebel A, Tsutsui S, Dunn JF, Kotra LP, Ousman SS, Wee Yong V, Stys PK (2018) Biochemically altered myelin triggers autoimmune demyelination. Proc Natl Acad Sci U S A 115:5528-5533 Available at: http://www.ncbi.nlm.nih.gov/pubmed/29728463 [Accessed March 5, 2019].

Cavanagh BL, Walker T, Norazit A, Meedeniya ACB (2011) Thymidine analogues for tracking DNA synthesis. Molecules 16:7980-7993 Available at: http://www.ncbi.nlm.nih.gov/pubmed/21921870 [Accessed March 27, 2019].

Chambers JS, Perrone-Bizzozero NI (2004) Altered myelination of the hippocampal formation in subjects with schizophrenia and bipolar disorder. Neurochem Res 29:2293-2302 Available at: http://www.ncbi.nlm.nih.gov/pubmed/15672553 [Accessed March 5, 2019].

Chang A, Tourtellotte WW, Rudick R, Trapp BD (2002) Premyelinating Oligodendrocytes in Chronic Lesions of Multiple Sclerosis. N Engl J Med 346:165-173 Available at: http://www.ncbi.nlm.nih.gov/pubmed/11796850 [Accessed April 4, 2019].

Chang K-J, Rasband MN (2013) Excitable Domains of Myelinated Nerves. In: Current topics in membranes, pp 159-192 Available at: http://www.ncbi.nlm.nih.gov/pubmed/24210430 [Accessed March 3, 2019].

Charles P, Reynolds R, Seilhean D, Rougon G, Aigrot MS, Niezgoda A, Zalc B, Lubetzki C (2002) Re-expression of PSA-NCAM by demyelinated axons: an inhibitor of remyelination in multiple sclerosis? Brain 125:1972-1979 Available at: http://www.ncbi.nlm.nih.gov/pubmed/12183343 [Accessed April 4, 2019].

Chen W-W, Chao Y-J, Chang W-H, Chan J-F, Hsu Y-HH (2018) Phosphatidylglycerol Incorporates into Cardiolipin to Improve Mitochondrial Activity and Inhibits Inflammation. Sci Rep 8:4919 Available at: http://www.nature.com/articles/s41598018-23190-z [Accessed April 7, 2019].

Chernoff GF (1981) Shiverer: an autosomal recessive mutant mouse with myelin deficiency. J Hered 72:128 Available at:

http://www.ncbi.nlm.nih.gov/pubmed/6168677 [Accessed March 5, 2019].

Chernousov MA, Stahl RC, Carey DJ (2013) Tetraspanins are involved in Schwann cell-axon interaction. J Neurosci Res 91:1419-1428 Available at: http://www.ncbi.nlm.nih.gov/pubmed/24038174 [Accessed April 6, 2019].

Chiu SY, Ritchie JM (1980) Potassium channels in nodal and internodal axonal membrane of mammalian myelinated fibres. Nature 284:170-171 Available at: http://www.ncbi.nlm.nih.gov/pubmed/6244497 [Accessed March 3, 2019].

Clarner T, Diederichs F, Berger K, Denecke B, Gan L, van der Valk P, Beyer C, Amor S, Kipp $M$ (2012) Myelin debris regulates inflammatory responses in an experimental 
demyelination animal model and multiple sclerosis lesions. Glia 60:1468-1480 Available at: http://www.ncbi.nlm.nih.gov/pubmed/22689449 [Accessed March 6, 2019].

Coetzee T, Fujita N, Dupree J, Shi R, Blight A, Suzuki K, Suzuki K, Popko B (1996) Myelination in the absence of galactocerebroside and sulfatide: normal structure with abnormal function and regional instability. Cell 86:209-219 Available at: http://www.ncbi.nlm.nih.gov/pubmed/8706126 [Accessed March 6, 2019].

Colman DR, Kreibich G, Frey AB, Sabatini DD (1982) Synthesis and incorporation of myelin polypeptides into CNS myelin. J Cell Biol 95:598-608 Available at: http://www.ncbi.nlm.nih.gov/pubmed/6183276 [Accessed March 4, 2019].

Corneliuson O, Berthold CH, Fredman P (1987) Isolation of myelinoid Marchi-positive bodies from normal rabbit spinal cord. Brain Res 416:43-53 Available at: http://www.ncbi.nlm.nih.gov/pubmed/2441811.

Czopka T, Ffrench-Constant C, Lyons DA (2013) Individual oligodendrocytes have only a few hours in which to generate new myelin sheaths in vivo. Dev Cell 25:599-609 Available at: http://www.ncbi.nlm.nih.gov/pubmed/23806617 [Accessed April 6, 2019].

Dawson RM, Gould RM (1976) Renewal of phospholipids in the myelin sheath. Adv Exp Med Biol 72:95-113 Available at: http://www.ncbi.nlm.nih.gov/pubmed/60049 [Accessed February 27, 2019].

Delassalle A, Zalc B, Lachapelle F, Raoul M, Collier P, Jacque C (1981) Regional distribution of myelin basic protein in the central nervous system of quaking, jimpy, and normal mice during development and aging. J Neurosci Res 6:303-313 Available at: http://www.ncbi.nlm.nih.gov/pubmed/6170761 [Accessed April 5, 2019].

Devaux JJ, Kleopa KA, Cooper EC, Scherer SS (2004) KCNQ2 Is a Nodal K+ Channel. J Neurosci 24:1236-1244 Available at:

http://www.ncbi.nlm.nih.gov/pubmed/14762142 [Accessed March 3, 2019].

Dimou L, Simon C, Kirchhoff F, Takebayashi H, Götz M (2008) Progeny of Olig2-expressing progenitors in the gray and white matter of the adult mouse cerebral cortex. J Neurosci 28:10434-10442 Available at:

http://www.ncbi.nlm.nih.gov/pubmed/18842903 [Accessed March 6, 2019].

Doolittle DP, Schweikart KM (1977) Myelin deficient, a new neurological mutant in the mouse. J Hered 68:331-332 Available at:

http://www.ncbi.nlm.nih.gov/pubmed/599283 [Accessed March 5, 2019].

Duflocq A, Le Bras B, Bullier E, Couraud F, Davenne M (2008) Nav1.1 is predominantly expressed in nodes of Ranvier and axon initial segments. Mol Cell Neurosci 39:180192 Available at: http://www.ncbi.nlm.nih.gov/pubmed/18621130 [Accessed March 3, 2019].

Duncan ID, Marik RL, Broman AT, Heidari M (2017) Thin myelin sheaths as the hallmark of remyelination persist over time and preserve axon function. Proc Natl Acad Sci 114:E9685-E9691 Available at: http://www.ncbi.nlm.nih.gov/pubmed/29078396 [Accessed March 6, 2019].

Dupree JL, Coetzee T, Blight A, Suzuki K, Popko B (1998) Myelin galactolipids are essential for proper node of Ranvier formation in the CNS. J Neurosci 18:1642-1649 Available at: http://www.ncbi.nlm.nih.gov/pubmed/9464989 [Accessed March 3, 2019].

Dyer CA, Philibotte TM, Wolf MK, Billings-Gagliardi S (1994) Myelin basic protein mediates 
extracellular signals that regulate microtubule stability in oligodendrocyte membrane sheets. J Neurosci Res 39:97-107 Available at: http://doi.wiley.com/10.1002/jnr.490390112 [Accessed March 6, 2019].

Elazar N, Vainshtein A, Golan N, Vijayaragavan B, Schaeren-Wiemers N, Eshed-Eisenbach Y, Peles E (2019) Axoglial Adhesion by Cadm4 Regulates CNS Myelination. Neuron 101:224-231.e5 Available at: http://www.ncbi.nlm.nih.gov/pubmed/30551998 [Accessed March 3, 2019].

Erwig MS, Hesse D, Jung RB, Uecker M, Kusch K, Tenzer S, Jahn O, Werner HB (2019) Myelin: Methods for Purification and Proteome Analysis. Methods Mol Biol 1936:3763 Available at: http://link.springer.com/10.1007/978-1-4939-9072-6_3 [Accessed March 17, 2019].

Fjell J, Hjelmström P, Hormuzdiar W, Milenkovic M, Aglieco F, Tyrrell L, Dib-Hajj S, Waxman SG, Black JA (2000) Localization of the tetrodotoxin-resistant sodium channel NaN in nociceptors. Neuroreport 11:199-202 Available at: http://www.ncbi.nlm.nih.gov/pubmed/10683857 [Accessed March 3, 2019].

Fornasiero EF et al. (2018) Precisely measured protein lifetimes in the mouse brain reveal differences across tissues and subcellular fractions. Nat Commun 9:4230 Available at: http://www.nature.com/articles/s41467-018-06519-0 [Accessed February 27, 2019].

Friede RL, Meier T, Diem M (1981) How is the exact length of an internode determined? J Neurol Sci 50:217-228 Available at: https://www.sciencedirect.com/science/article/pii/0022510X81901684 [Accessed April 5, 2019].

Fünfschilling U, Supplie LM, Mahad D, Boretius S, Saab AS, Edgar J, Brinkmann BG, Kassmann CM, Tzvetanova ID, Möbius W, Diaz F, Meijer D, Suter U, Hamprecht B, Sereda MW, Moraes CT, Frahm J, Goebbels S, Nave K-A (2012) Glycolytic oligodendrocytes maintain myelin and long-term axonal integrity. Nature 485:517521 Available at: http://www.ncbi.nlm.nih.gov/pubmed/22622581 [Accessed February 27, 2019].

García-Mateo N, Pascua-Maestro R, Pérez-Castellanos A, Lillo C, Sanchez D, Ganfornina MD (2018) Myelin extracellular leaflet compaction requires apolipoprotein D membrane management to optimize lysosomal-dependent recycling and glycocalyx removal. Glia 66:670-687 Available at: http://doi.wiley.com/10.1002/glia.23274 [Accessed April $6,2019]$.

Gaultier A, Wu X, Le Moan N, Takimoto S, Mukandala G, Akassoglou K, Campana WM, Gonias SL (2009) Low-density lipoprotein receptor-related protein 1 is an essential receptor for myelin phagocytosis. J Cell Sci 122:1155-1162 Available at: http://jcs.biologists.org/cgi/doi/10.1242/jcs.040717 [Accessed February 27, 2019].

Ghasemlou N, Jeong SY, Lacroix S, David S (2007) T cells contribute to lysophosphatidylcholine-induced macrophage activation and demyelination in the CNS. Glia 55:294-302 Available at: http://www.ncbi.nlm.nih.gov/pubmed/17096403 [Accessed April 5, 2019].

Giacci MK, Bartlett CA, Huynh M, Kilburn MR, Dunlop SA, Fitzgerald M (2018) Three dimensional electron microscopy reveals changing axonal and myelin morphology along normal and partially injured optic nerves. Sci Rep 8:3979 Available at: http://www.nature.com/articles/s41598-018-22361-2 [Accessed March 26, 2019].

Gledhill RF, McDonald WI (1977) Morphological characteristics of central demyelination 
and remyelination: A single-fiber study. Ann Neurol 1:552-560 Available at: http://doi.wiley.com/10.1002/ana.410010607 [Accessed March 6, 2019].

Gould RM, Byrd AL, Barbarese E (1995) The number of Schmidt-Lanterman incisures is more than doubled in shiverer PNS myelin sheaths. J Neurocytol 24:85-98 Available at: http://www.ncbi.nlm.nih.gov/pubmed/7745445 [Accessed March 5, 2019].

Graziano ACE, Cardile V (2015) History, genetic, and recent advances on Krabbe disease. Gene 555:2-13 Available at: https://www.sciencedirect.com/science/article/pii/S0378111914010841?via\%253 Dihub [Accessed March 7, 2019].

Griffiths I, Klugmann M, Anderson T, Yool D, Thomson C, Schwab MH, Schneider A, Zimmermann F, McCulloch M, Nadon N, Nave KA (1998) Axonal swellings and degeneration in mice lacking the major proteolipid of myelin. Science (80-) 280:1610-1613 Available at: http://www.ncbi.nlm.nih.gov/pubmed/9616125.

Harauz G, Libich DS (2009) The classic basic protein of myelin--conserved structural motifs and the dynamic molecular barcode involved in membrane adhesion and protein-protein interactions. Curr Protein Pept Sci 10:196-215 Available at: http://www.ncbi.nlm.nih.gov/pubmed/19519451 [Accessed March 4, 2019].

Harrison BM, McDonald WI, Ochoa J (1972) Remyelination in the central diphtheria toxin lesion. J Neurol Sci 17:293-302 Available at:

https://www.sciencedirect.com/science/article/pii/0022510X72900342 [Accessed March 6, 2019].

Hartline DK, Colman DR (2007) Rapid conduction and the evolution of giant axons and myelinated fibers. Curr Biol 17:R29--35 Available at: http://www.ncbi.nlm.nih.gov/pubmed/17208176.

Hibbits N, Yoshino J, Le TQ, Armstrong RC (2012) Astrogliosis during acute and chronic cuprizone demyelination and implications for remyelination. ASN Neuro 4:393-408 Available at: http://www.ncbi.nlm.nih.gov/pubmed/23025787.

Hildebrand C, Aldskogius H (1976) Electron-microscopic identification of Marchi-positive bodies and argyrophilic granules in the spinal cord white matter of the guinea pig. J Comp Neurol 170:191-203 Available at: http://doi.wiley.com/10.1002/cne.901700205 [Accessed March 22, 2019].

Hildebrand C, Bowe CM, Remahl IN (1994) Myelination and myelin sheath remodelling in normal and pathological PNS nerve fibres. Prog Neurobiol 43:85-141 Available at: http://www.ncbi.nlm.nih.gov/pubmed/7972854 [Accessed February 27, 2019].

Hildebrand C, Remahl S, Persson H, Bjartmar C (1993) Myelinated nerve fibres in the CNS. Prog Neurobiol 40:319-384 Available at: http://www.ncbi.nlm.nih.gov/pubmed/8441812 [Accessed February 27, 2019].

Hill CM, Bates IR, White GF, Hallett FR, Harauz G (2002) Effects of the osmolyte trimethylamine-N-oxide on conformation, self-association, and two-dimensional crystallization of myelin basic protein. J Struct Biol 139:13-26 Available at: http://www.ncbi.nlm.nih.gov/pubmed/12372316 [Accessed March 4, 2019].

Hill RA, Li AM, Grutzendler J (2018) Lifelong cortical myelin plasticity and age-related degeneration in the live mammalian brain. Nat Neurosci 21:683-695 Available at: http://www.ncbi.nlm.nih.gov/pubmed/29556031 [Accessed March 22, 2019].

Hobson GM, Kamholz J (1993) PLP1-Related Disorders. University of Washington, Seattle. Available at: http://www.ncbi.nlm.nih.gov/pubmed/20301361 [Accessed March 5, 
2019].

Huang JK (2005) Glial Membranes at the Node of Ranvier Prevent Neurite Outgrowth. Science (80- ) 310:1813-1817 Available at: http://www.sciencemag.org/cgi/doi/10.1126/science.1118313 [Accessed April 14, 2019].

Iglesias A, Bauer J, Litzenburger T, Schubart A, Linington C (2001) T- and B-cell responses to myelin oligodendrocyte glycoprotein in experimental autoimmune encephalomyelitis and multiple sclerosis. Glia 36:220-234 Available at: http://doi.wiley.com/10.1002/glia.1111 [Accessed March 6, 2019].

Isbister GK, Kiernan MC (2005) Neurotoxic marine poisoning. Lancet Neurol 4:219-228 Available at: http://www.ncbi.nlm.nih.gov/pubmed/15778101 [Accessed March 3, 2019].

Jahn O, Tenzer S, Bartsch N, Patzig J, Werner HB (2013) Myelin Proteome Analysis: Methods and Implications for the Myelin Cytoskeleton. In, pp 335-353. Springer Science + Business Media. Available at: http://link.springer.com/10.1007/978-162703-266-7_15 [Accessed March 17, 2019].

Jahn O, Tenzer S, Werner HB (2009) Myelin proteomics: molecular anatomy of an insulating sheath. Mol Neurobiol 40:55-72 Available at: http://www.ncbi.nlm.nih.gov/pubmed/19452287 [Accessed March 4, 2019].

Jäkel S, Agirre E, Mendanha Falcão A, van Bruggen D, Lee KW, Knuesel I, Malhotra D, ffrench-Constant C, Williams A, Castelo-Branco G (2019) Altered human oligodendrocyte heterogeneity in multiple sclerosis. Nature 566:543-547 Available at: http://www.nature.com/articles/s41586-019-0903-2 [Accessed March 4, 2019].

Jay TR et al. (2015) TREM2 deficiency eliminates TREM2 + inflammatory macrophages and ameliorates pathology in Alzheimer's disease mouse models. J Exp Med 212:287-295 Available at: http://www.ncbi.nlm.nih.gov/pubmed/25732305 [Accessed February $27,2019]$.

Johns TG, Bernard CCA (1999) The Structure and Function of Myelin Oligodendrocyte Glycoprotein. J Neurochem 72:1-9 Available at:

http://doi.wiley.com/10.1046/j.1471-4159.1999.0720001.x [Accessed March 6, 2019].

Kamholz J, de Ferra F, Puckett C, Lazzarini R (1986) Identification of three forms of human myelin basic protein by cDNA cloning. Proc Natl Acad Sci U S A 83:4962-4966 Available at: http://www.ncbi.nlm.nih.gov/pubmed/2425357 [Accessed March 4, 2019].

Kandel ER, Schwartz JH, Jessell TM (2012) Principles of Neural Science, Fifth Edition 2012. McGraw-Hill Education.

Kanehisa M, Furumichi M, Tanabe M, Sato Y, Morishima K (2017) KEGG: new perspectives on genomes, pathways, diseases and drugs. Nucleic Acids Res 45:D353-D361 Available at: http://www.ncbi.nlm.nih.gov/pubmed/27899662 [Accessed March 17, 2019].

Kanehisa M, Goto S (2000) KEGG: kyoto encyclopedia of genes and genomes. Nucleic Acids Res 28:27-30 Available at: http://www.ncbi.nlm.nih.gov/pubmed/10592173 [Accessed March 17, 2019].

Kanehisa M, Sato Y, Furumichi M, Morishima K, Tanabe M (2019) New approach for understanding genome variations in KEGG. Nucleic Acids Res 47:D590-D595 
Available at: http://www.ncbi.nlm.nih.gov/pubmed/30321428 [Accessed March 17, 2019].

Kang SH, Fukaya M, Yang JK, Rothstein JD, Bergles DE (2010) NG2+ CNS Glial Progenitors Remain Committed to the Oligodendrocyte Lineage in Postnatal Life and following Neurodegeneration. Neuron 68:668-681 Available at: http://www.ncbi.nlm.nih.gov/pubmed/21092857 [Accessed March 6, 2019].

Katsuki M, Sato M, Kimura M, Yokoyama M, Kobayashi K, Nomura T (1988) Conversion of normal behavior to shiverer by myelin basic protein antisense cDNA in transgenic mice. Science 241:593-595 Available at: http://www.ncbi.nlm.nih.gov/pubmed/2456614 [Accessed March 5, 2019].

Kessaris N, Fogarty M, Iannarelli P, Grist M, Wegner M, Richardson WD (2006) Competing waves of oligodendrocytes in the forebrain and postnatal elimination of an embryonic lineage. Nat Neurosci 9:173-179 Available at: http://www.nature.com/articles/nn1620 [Accessed March 7, 2019].

Kimura M, Sato M, Akatsuka A, Nozawa-Kimura S, Takahashi R, Yokoyama M, Nomura T, Katsuki M (1989) Restoration of myelin formation by a single type of myelin basic protein in transgenic shiverer mice. Proc Natl Acad Sci U S A 86:5661-5665 Available at: http://www.ncbi.nlm.nih.gov/pubmed/2473474 [Accessed March 4, 2019].

King CH, Lancaster E, Salomon D, Peles E, Scherer SS (2014) Kv7.2 regulates the function of peripheral sensory neurons. J Comp Neurol 522:3262-3280 Available at: http://www.ncbi.nlm.nih.gov/pubmed/24687876 [Accessed March 3, 2019].

Kirkpatrick LL, Witt AS, Payne HR, Shine HD, Brady ST (2001) Changes in microtubule stability and density in myelin-deficient shiverer mouse CNS axons. J Neurosci 21:2288-2297 Available at: http://www.ncbi.nlm.nih.gov/pubmed/11264304 [Accessed April 6, 2019].

Kirschner DA, Ganser AL (1980) Compact myelin exists in the absence of basic protein in the shiverer mutant mouse. Nature 283:207-210 Available at: http://www.ncbi.nlm.nih.gov/pubmed/6153124 [Accessed March 4, 2019].

Kiryu-Seo S, Ohno N, Kidd GJ, Komuro H, Trapp BD (2010) Demyelination increases axonal stationary mitochondrial size and the speed of axonal mitochondrial transport. J Neurosci 30:6658-6666 Available at: http://www.ncbi.nlm.nih.gov/pubmed/20463228 [Accessed April 6, 2019].

Koenning M, Jackson S, Hay CM, Faux C, Kilpatrick TJ, Willingham M, Emery B (2012) Myelin gene regulatory factor is required for maintenance of myelin and mature oligodendrocyte identity in the adult CNS. J Neurosci 32:12528-12542 Available at: http://www.ncbi.nlm.nih.gov/pubmed/22956843 [Accessed March 4, 2019].

Kordeli E, Lambert S, Bennett V (1995) AnkyrinG. A new ankyrin gene with neural-specific isoforms localized at the axonal initial segment and node of Ranvier. J Biol Chem 270:2352-2359 Available at: http://www.ncbi.nlm.nih.gov/pubmed/7836469 [Accessed March 3, 2019].

Kornek B, Storch MK, Weissert R, Wallstroem E, Stefferl A, Olsson T, Linington C, Schmidbauer M, Lassmann H (2000) Multiple Sclerosis and Chronic Autoimmune Encephalomyelitis. Am J Pathol 157:267-276 Available at: http://www.ncbi.nlm.nih.gov/pubmed/10880396 [Accessed March 7, 2019].

Korogod N, Petersen CCH, Knott GW (2015) Ultrastructural analysis of adult mouse neocortex comparing aldehyde perfusion with cryo fixation. Elife. 
Kotter MR, Li W-W, Zhao C, Franklin RJM (2006) Myelin Impairs CNS Remyelination by Inhibiting Oligodendrocyte Precursor Cell Differentiation. J Neurosci 26:328-332 Available at: http://www.ncbi.nlm.nih.gov/pubmed/16399703 [Accessed February 27, 2019].

Kristensson K, Zeller NK, Dubois-Dalcq ME, Lazzarini RA (1986) Expression of myelin basic protein gene in the developing rat brain as revealed by in situ hybridization. J Histochem Cytochem 34:467-473 Available at: http://www.ncbi.nlm.nih.gov/pubmed/2419396 [Accessed March 4, 2019].

Landry CF, Ellison J, Skinner E, Campagnoni AT (1997) Golli-MBP proteins mark the earliest stages of fiber extension and terminal arboration in the mouse peripheral nervous system. J Neurosci Res 50:265-271 Available at: http://www.ncbi.nlm.nih.gov/pubmed/9373036 [Accessed March 4, 2019].

Lappe-Siefke C, Goebbels S, Gravel M, Nicksch E, Lee J, Braun PE, Griffiths IR, Nave K-A (2003) Disruption of Cnp1 uncouples oligodendroglial functions in axonal support and myelination. Nat Genet 33:366-374 Available at: http://www.nature.com/articles/ng1095 [Accessed March 6, 2019].

Larocca JN, Norton WT (2007) Isolation of myelin. Curr Protoc Cell Biol Chapter 3:Unit3.25 Available at: http://www.ncbi.nlm.nih.gov/pubmed/18228513.

Lee Y, Morrison BM, Li Y, Lengacher S, Farah MH, Hoffman PN, Liu Y, Tsingalia A, Jin L, Zhang P-W, Pellerin L, Magistretti PJ, Rothstein JD (2012) Oligodendroglia metabolically support axons and contribute to neurodegeneration. Nature 487:443448 Available at: http://www.ncbi.nlm.nih.gov/pubmed/22801498 [Accessed February 27, 2019].

Leone DP, Genoud S, Atanasoski S, Grausenburger R, Berger P, Metzger D, Macklin WB, Chambon P, Suter U (2003) Tamoxifen-inducible glia-specific Cre mice for somatic mutagenesis in oligodendrocytes and Schwann cells. Mol Cell Neurosci 22:430-440 Available at: http://www.ncbi.nlm.nih.gov/pubmed/12727441 [Accessed March 20, 2019].

Li C, Tropak MB, Gerlai R, Clapoff S, Abramow-Newerly W, Trapp B, Peterson A, Roder J (1994) Myelination in the absence of myelin-associated glycoprotein. Nature 369:747-750 Available at: http://www.ncbi.nlm.nih.gov/pubmed/7516497 [Accessed March 6, 2019].

Libich DS, Harauz G (2008) Backbone Dynamics of the 18.5kDa Isoform of Myelin Basic Protein Reveals Transient $\alpha$-Helices and a Calmodulin-Binding Site. Biophys J 94:4847-4866 Available at: http://www.ncbi.nlm.nih.gov/pubmed/18326633 [Accessed March 4, 2019].

Liou W, Geuze HJ, Slot JW (1996) Improving structural integrity of cryosections for immunogold labeling. Histochem Cell Biol.

Lüders KA, Nessler S, Kusch K, Patzig J, Jung RB, Möbius W, Nave K-A, Werner HB (2019) Maintenance of high proteolipid protein level in adult central nervous system myelin is required to preserve the integrity of myelin and axons. Glia 67:634-649 Available at: http://doi.wiley.com/10.1002/glia.23549 [Accessed March 6, 2019].

Lüders KA, Patzig J, Simons M, Nave K-A, Werner HB (2017) Genetic dissection of oligodendroglial and neuronal Plp1 function in a novel mouse model of spastic paraplegia type 2. Glia 65:1762-1776 Available at: http://doi.wiley.com/10.1002/glia.23193 [Accessed April 8, 2019]. 
Magrassi L, Leto K, Rossi F (2013) Lifespan of neurons is uncoupled from organismal lifespan. Proc Natl Acad Sci U S A 110:4374-4379 Available at:

http://www.ncbi.nlm.nih.gov/pubmed/23440189.

Majava V, Wang C, Myllykoski M, Kangas SM, Kang SU, Hayashi N, Baumgärtel P, Heape AM, Lubec G, Kursula P (2010) Structural analysis of the complex between calmodulin and full-length myelin basic protein, an intrinsically disordered molecule. Amino Acids 39:59-71 Available at: http://www.ncbi.nlm.nih.gov/pubmed/19855925 [Accessed March 4, 2019].

Martini R, Mohajeri MH, Kasper S, Giese KP, Schachner M (1995) Mice doubly deficient in the genes for $\mathrm{P} 0$ and myelin basic protein show that both proteins contribute to the formation of the major dense line in peripheral nerve myelin. J Neurosci 15:44884495 Available at: http://www.ncbi.nlm.nih.gov/pubmed/7540676 [Accessed March 4, 2019].

Mathews ES, Mawdsley DJ, Walker M, Hines JH, Pozzoli M, Appel B (2014) Mutation of 3Hydroxy-3-Methylglutaryl CoA Synthase I Reveals Requirements for Isoprenoid and Cholesterol Synthesis in Oligodendrocyte Migration Arrest, Axon Wrapping, and Myelin Gene Expression. J Neurosci 34:3402-3412 Available at: http://www.ncbi.nlm.nih.gov/pubmed/24573296 [Accessed February 27, 2019].

Mathisen PM, Johnson JM, Kawczak JA (1997) Stabilization of myelin mRNAs as measured in a brain slice system. J Neurosci Res 50:1030-1039 Available at:

http://doi.wiley.com/10.1002/\%28SICI\%2910974547\%2819971215\%2950\%3A6\%3C1030\%3A\%3AAID-JNR14\%3E3.0.C0\%3B2-8 [Accessed April 15, 2019].

Matsushima GK, Morell P (2001) The neurotoxicant, cuprizone, as a model to study demyelination and remyelination in the central nervous system. Brain Pathol 11:107-116 Available at: http://www.ncbi.nlm.nih.gov/pubmed/11145196 [Accessed March 7, 2019].

Maxfield FR, van Meer G (2010) Cholesterol, the central lipid of mammalian cells. Curr Opin Cell Biol 22:422-429 Available at: http://www.ncbi.nlm.nih.gov/pubmed/20627678 [Accessed February 27, 2019].

McKenzie IA, Ohayon D, Li H, Paes de Faria J, Emery B, Tohyama K, Richardson WD (2014) Motor skill learning requires active central myelination. Science (80- ) 346:318-322 Available at: http://www.ncbi.nlm.nih.gov/pubmed/25324381 [Accessed March 4, 2019].

Mi S et al. (2007) LINGO-1 antagonist promotes spinal cord remyelination and axonal integrity in MOG-induced experimental autoimmune encephalomyelitis. Nat Med 13:1228-1233 Available at: http://www.ncbi.nlm.nih.gov/pubmed/17906634 [Accessed April 4, 2019].

Mi S et al. (2009) Promotion of central nervous system remyelination by induced differentiation of oligodendrocyte precursor cells. Ann Neurol 65:304-315 Available at: http://doi.wiley.com/10.1002/ana.21581 [Accessed April 4, 2019].

Mi S, Miller RH, Lee X, Scott ML, Shulag-Morskaya S, Shao Z, Chang J, Thill G, Levesque M, Zhang M, Hession C, Sah D, Trapp B, He Z, Jung V, McCoy JM, Pepinsky RB (2005) LINGO-1 negatively regulates myelination by oligodendrocytes. Nat Neurosci 8:745751 Available at: http://www.ncbi.nlm.nih.gov/pubmed/15895088 [Accessed April $4,2019]$.

Mikula S, Binding J, Denk W (2012) Staining and embedding the whole mouse brain for 
electron microscopy. Nat Methods.

Mills EA, Davis CO, Bushong EA, Boassa D, Kim K-Y, Ellisman MH, Marsh-Armstrong N (2015) Astrocytes phagocytose focal dystrophies from shortening myelin segments in the optic nerve of Xenopus laevis at metamorphosis. Proc Natl Acad Sci U S A 112:10509-10514 Available at: http://www.pnas.org/lookup/doi/10.1073/pnas.1506486112 [Accessed April 5, 2019].

Min Y, Kristiansen K, Boggs JM, Husted C, Zasadzinski JA, Israelachvili J (2009) Interaction forces and adhesion of supported myelin lipid bilayers modulated by myelin basic protein. Proc Natl Acad Sci 106:3154-3159 Available at: http://www.ncbi.nlm.nih.gov/pubmed/19218452 [Accessed March 4, 2019].

Mizuhira B, Ozawa H (1967) On the fine structure of nerve myelin by means of glutaraldehyde fixation. J Electron Microsc (Tokyo) 16:169-172 Available at: http://www.ncbi.nlm.nih.gov/pubmed/6079082 [Accessed March 4, 2019].

Möbius W, Cooper B, Kaufmann WA, Imig C, Ruhwedel T, Snaidero N, Saab AS, Varoqueaux F (2010) Electron microscopy of the mouse central nervous system.

Möbius W, Nave K-A, Werner HB (2016) Electron microscopy of myelin: Structure preservation by high-pressure freezing. Brain Res 1641:92-100 Available at: http://www.ncbi.nlm.nih.gov/pubmed/26920467.

Morell P, Quarles RH (1999) Characteristic Composition of Myelin. Available at: https://www.ncbi.nlm.nih.gov/books/NBK28221/ [Accessed March 5, 2019].

Morizawa YM, Hirayama Y, Ohno N, Shibata S, Shigetomi E, Sui Y, Nabekura J, Sato K, Okajima F, Takebayashi H, Okano H, Koizumi S (2017) Reactive astrocytes function as phagocytes after brain ischemia via ABCA1-mediated pathway. Nat Commun 8:28 Available at: http://www.ncbi.nlm.nih.gov/pubmed/28642575 [Accessed February $27,2019]$.

Morrison JH, Hof PR (1997) Life and death of neurons in the aging brain. Science 278:412419 Available at: http://www.ncbi.nlm.nih.gov/pubmed/9334292.

Mortz E, Krogh TN, Vorum H, Görg A (2001) Improved silver staining protocols for high sensitivity protein identification using matrix-assisted laser desorption/ionizationtime of flight analysis. Proteomics 1:1359-1363 Available at: http://www.ncbi.nlm.nih.gov/pubmed/11922595 [Accessed March 17, 2019].

Moscarello MA, Mastronardi FG, Wood DD (2007) The role of citrullinated proteins suggests a novel mechanism in the pathogenesis of multiple sclerosis. Neurochem Res 32:251-256 Available at: http://www.ncbi.nlm.nih.gov/pubmed/17031564 [Accessed April 4, 2019].

Moscarello MA, Wood DD, Ackerley C, Boulias C (1994) Myelin in multiple sclerosis is developmentally immature. J Clin Invest 94:146-154 Available at: http://www.ncbi.nlm.nih.gov/pubmed/7518827 [Accessed March 5, 2019].

Muffat J, Walker DW (2010) Apolipoprotein D: an overview of its role in aging and agerelated diseases. Cell Cycle 9:269-273 Available at: http://www.ncbi.nlm.nih.gov/pubmed/20023409 [Accessed April 6, 2019].

Napoli I, Neumann H (2010) Protective effects of microglia in multiple sclerosis. Exp Neurol 225:24-28 Available at: http://www.ncbi.nlm.nih.gov/pubmed/19409897 [Accessed February 27, 2019]. 
Narayan K, Danielson CM, Lagarec K, Lowekamp BC, Coffman P, Laquerre A, Phaneuf MW, Hope TJ, Subramaniam S (2014) Multi-resolution correlative focused ion beam scanning electron microscopy: Applications to cell biology. J Struct Biol.

Nave K-A, Werner HB (2014) Myelination of the Nervous System: Mechanisms and Functions. Annu Rev Cell Dev Biol 30:503-533 Available at: http://www.ncbi.nlm.nih.gov/pubmed/25288117 [Accessed February 27, 2019].

Nawaz S, Sánchez P, Schmitt S, Snaidero N, Mitkovski M, Velte C, Brückner BR, Alexopoulos I, Czopka T, Jung SY, Rhee JS, Janshoff A, Witke W, Schaap IAT, Lyons DA, Simons M (2015) Actin filament turnover drives leading edge growth during myelin sheath formation in the central nervous system. Dev Cell 34:139-151 Available at: http://www.ncbi.nlm.nih.gov/pubmed/26166299 [Accessed March 3, 2019].

Nawaz S, Schweitzer J, Jahn O, Werner HB (2013) Molecular evolution of myelin basic protein, an abundant structural myelin component. Glia 61:1364-1377 Available at: http://www.ncbi.nlm.nih.gov/pubmed/24040667.

Neumann H, Kotter MR, Franklin RJM (2009) Debris clearance by microglia: an essential link between degeneration and regeneration. Brain 132:288-295 Available at: http://www.ncbi.nlm.nih.gov/pubmed/18567623 [Accessed March 3, 2019].

Noebels JL, Marcom PK, Jalilian-Tehrani MH (1991) Sodium channel density in hypomyelinated brain increased by myelin basic protein gene deletion. Nature 352:431-434 Available at: http://www.ncbi.nlm.nih.gov/pubmed/1713650 [Accessed March 5, 2019].

Norton WT, Autili LA (1965) The chemical composition of Bovine. Ann N Y Acad Sci 122:77-85 Available at: http://www.ncbi.nlm.nih.gov/pubmed/14313516 [Accessed March 5, 2019].

Norton WT, Poduslo SE (1973) Myelination in rat brain: changes in myelin composition during brain maturation. J Neurochem 21:759-773 Available at: http://www.ncbi.nlm.nih.gov/pubmed/4754856 [Accessed February 27, 2019].

Ohler B, Graf K, Bragg R, Lemons T, Coe R, Genain C, Israelachvili J, Husted C (2004) Role of lipid interactions in autoimmune demyelination. Biochim Biophys Acta - Mol Basis Dis 1688:10-17 Available at:

https://www.sciencedirect.com/science/article/pii/S0925443903001650 [Accessed April 12, 2019].

Okano H, Miura M, Moriguchi A, Ikenaka K, Tsukada Y, Mikoshiba K (1987) Inefficient transcription of the myelin basic protein gene possibly causes hypomyelination in myelin-deficient mutant mice. J Neurochem 48:470-476 Available at: http://www.ncbi.nlm.nih.gov/pubmed/2432182 [Accessed March 5, 2019].

Ono J, Harada K, Yamamoto T, Onoe S, Okada S (1994) Delayed myelination in a patient with 18q- syndrome. Pediatr Neurol 11:64-67 Available at: https://www.sciencedirect.com/science/article/pii/0887899494900957 [Accessed March 5, 2019].

Özbalci C, Sachsenheimer T, Brügger B (2013) Quantitative Analysis of Cellular Lipids by Nano-Electrospray Ionization Mass Spectrometry. In: Methods in molecular biology (Clifton, N.J.), pp 3-20 Available at: http://www.ncbi.nlm.nih.gov/pubmed/23996167 [Accessed March 17, 2019].

Paez PM, Spreuer V, Handley V, Feng J-M, Campagnoni C, Campagnoni AT (2007) Increased Expression of Golli Myelin Basic Proteins Enhances Calcium Influx into 
Oligodendroglial Cells. J Neurosci 27:12690-12699 Available at:

http://www.ncbi.nlm.nih.gov/pubmed/18003849 [Accessed March 4, 2019].

Papadopoulos MC, Verkman A (2012) Aquaporin 4 and neuromyelitis optica. Lancet Neurol 11:535-544 Available at:

https://www.sciencedirect.com/science/article/pii/S1474442212701333?via\%253

Dihub [Accessed March 7, 2019].

Parlapani E, Schmitt A, Erdmann A, Bernstein H-G, Breunig B, Gruber O, Petroianu G, von Wilmsdorff M, Schneider-Axmann T, Honer W, Falkai P (2009) Association between myelin basic protein expression and left entorhinal cortex pre-alpha cell layer disorganization in schizophrenia. Brain Res 1301:126-134 Available at: http://www.ncbi.nlm.nih.gov/pubmed/19747901 [Accessed March 5, 2019].

Patzig J, Erwig MS, Tenzer S, Kusch K, Dibaj P, Möbius W, Goebbels S, Schaeren-Wiemers N, Nave K-A, Werner HB (2016) Septin/anillin filaments scaffold central nervous system myelin to accelerate nerve conduction. Elife 5 Available at: http://www.ncbi.nlm.nih.gov/pubmed/27504968 [Accessed March 6, 2019].

Pedraza L (1997) Nuclear transport of myelin basic protein. J Neurosci Res 50:258-264 Available at: http://www.ncbi.nlm.nih.gov/pubmed/9373035 [Accessed March 4, 2019].

Pellerin L, Magistretti PJ (1994) Glutamate uptake into astrocytes stimulates aerobic glycolysis: a mechanism coupling neuronal activity to glucose utilization. Proc Natl Acad Sci U S A 91:10625-10629 Available at: http://www.ncbi.nlm.nih.gov/pubmed/7938003.

Pelvig DP, Pakkenberg H, Stark AK, Pakkenberg B (2008) Neocortical glial cell numbers in human brains. Neurobiol Aging 29:1754-1762 Available at: http://www.ncbi.nlm.nih.gov/pubmed/17544173 [Accessed March 22, 2019].

Perge JA, Koch K, Miller R, Sterling P, Balasubramanian V (2009) How the optic nerve allocates space, energy capacity, and information. J Neurosci 29:7917-7928 Available at: http://www.ncbi.nlm.nih.gov/pubmed/19535603 [Accessed February 28, 2019].

Persson H, Berthold C-H (1991) Cellular relationships of paranodal Marchi-positive bodies studied with monoclonal antibodies against partially degraded CNS myelin fragments. J Neurocytol 20:277-289 Available at: http://link.springer.com/10.1007/BF01235545 [Accessed March 22, 2019].

Persson H, Berthold C-H, Rydmark M, Fabricius C (1992) Metabolic relationships between proteins of myelin and paranodally shedded, partially degraded myelin fragments in the rabbit CNS. J Neurosci Res 33:310-318 Available at:

http://www.ncbi.nlm.nih.gov/pubmed/1453493 [Accessed March 22, 2019].

Peters A, Sethares C (2002) Aging and the myelinated fibers in prefrontal cortex and corpus callosum of the monkey. J Comp Neurol 442:277-291 Available at: http://www.ncbi.nlm.nih.gov/pubmed/11774342.

Peterson LK, Fujinami RS (2007) Inflammation, demyelination, neurodegeneration and neuroprotection in the pathogenesis of multiple sclerosis. J Neuroimmunol 184:3744 Available at: http://www.ncbi.nlm.nih.gov/pubmed/17196667 [Accessed March 26, 2019].

Pfeifenbring S, Nessler S, Wegner C, Stadelmann C, Brück W (2015) Remyelination After Cuprizone-Induced Demyelination Is Accelerated in Juvenile Mice. J Neuropathol Exp Neurol 74:756-766 Available at: http://www.ncbi.nlm.nih.gov/pubmed/26115190 
[Accessed April 4, 2019].

Pfeiffer SE, Warrington AE, Bansal R (1993) The oligodendrocyte and its many cellular processes. Trends Cell Biol 3:191-197 Available at: http://www.ncbi.nlm.nih.gov/pubmed/14731493 [Accessed February 27, 2019].

Pfrieger FW, Ungerer N (2011) Cholesterol metabolism in neurons and astrocytes. Prog Lipid Res 50:357-371 Available at: http://www.ncbi.nlm.nih.gov/pubmed/21741992 [Accessed April 4, 2019].

Philips T, Rothstein JD (2017) Oligodendroglia: metabolic supporters of neurons. J Clin Invest 127:3271-3280 Available at: http://www.ncbi.nlm.nih.gov/pubmed/28862639 [Accessed February 27, 2019].

Plemel JR, Michaels NJ, Weishaupt N, Caprariello A V., Keough MB, Rogers JA, Yukseloglu A, Lim J, Patel V V., Rawji KS, Jensen SK, Teo W, Heyne B, Whitehead SN, Stys PK, Yong VW (2018) Mechanisms of lysophosphatidylcholine-induced demyelination: A primary lipid disrupting myelinopathy. Glia 66:327-347 Available at: http://doi.wiley.com/10.1002/glia.23245 [Accessed March 7, 2019].

Poggi G, Boretius S, Möbius W, Moschny N, Baudewig J, Ruhwedel T, Hassouna I, Wieser GL, Werner HB, Goebbels S, Nave K-A, Ehrenreich H (2016) Cortical network dysfunction caused by a subtle defect of myelination. Glia 64:2025-2040 Available at: http://www.ncbi.nlm.nih.gov/pubmed/27470661 [Accessed March 5, 2019].

Poliani PL, Wang Y, Fontana E, Robinette ML, Yamanishi Y, Gilfillan S, Colonna M (2015) TREM2 sustains microglial expansion during aging and response to demyelination. J Clin Invest 125:2161-2170 Available at: http://www.jci.org/articles/view/77983 [Accessed February 27, 2019].

Pöllinger B, Krishnamoorthy G, Berer K, Lassmann H, Bösl MR, Dunn R, Domingues HS, Holz A, Kurschus FC, Wekerle H (2009) Spontaneous relapsing-remitting EAE in the SJL/J mouse: MOG-reactive transgenic T cells recruit endogenous MOG-specific B cells. J Exp Med 206:1303-1316 Available at: http://www.ncbi.nlm.nih.gov/pubmed/19487416 [Accessed March 6, 2019].

Ponath G, Ramanan S, Mubarak M, Housley W, Lee S, Sahinkaya FR, Vortmeyer A, Raine CS, Pitt D (2017) Myelin phagocytosis by astrocytes after myelin damage promotes lesion pathology. Brain 140:399-413 Available at:

http://www.ncbi.nlm.nih.gov/pubmed/28007993 [Accessed April 5, 2019].

Pribyl TM, Campagnoni CW, Kampf K, Kashima T, Handley VW, McMahon J, Campagnoni AT (1993) The human myelin basic protein gene is included within a 179-kilobase transcription unit: expression in the immune and central nervous systems. Proc Natl Acad Sci U S A 90:10695-10699 Available at:

http://www.ncbi.nlm.nih.gov/pubmed/7504278 [Accessed March 4, 2019].

Price JC, Guan S, Burlingame A, Prusiner SB, Ghaemmaghami S (2010) Analysis of proteome dynamics in the mouse brain. Proc Natl Acad Sci U S A 107:14508-14513 Available at: http://www.ncbi.nlm.nih.gov/pubmed/20699386 [Accessed February $27,2019]$.

Privat A, Jacque C, Bourre JM, Dupouey P, Baumann N (1979) Absence of the major dense line in myelin of the mutant mouse \&quot;shiverer\&quot;. Neurosci Lett 12:107-112 Available at: http://www.ncbi.nlm.nih.gov/pubmed/460693 [Accessed March 5, 2019].

Pronker MF, Lemstra S, Snijder J, Heck AJR, Thies-Weesie DME, Pasterkamp RJ, Janssen BJC 
(2016) Structural basis of myelin-associated glycoprotein adhesion and signalling. Nat Commun 7:13584 Available at: http://www.nature.com/articles/ncomms13584 [Accessed April 8, 2019].

Psachoulia K, Jamen F, Young KM, Richardson WD (2009) Cell cycle dynamics of NG2 cells in the postnatal and ageing brain. Neuron Glia Biol 5:57 Available at: http://www.ncbi.nlm.nih.gov/pubmed/20346197 [Accessed March 6, 2019].

Quarles RH (2007) Myelin-associated glycoprotein (MAG): past, present and beyond. J Neurochem 0:070214184024009-??? Available at: http://doi.wiley.com/10.1111/j.1471-4159.2006.04319.x [Accessed March 6, 2019].

Raasakka A, Ruskamo S, Kowal J, Barker R, Baumann A, Martel A, Tuusa J, Myllykoski M, Bürck J, Ulrich AS, Stahlberg H, Kursula P (2017) Membrane Association Landscape of Myelin Basic Protein Portrays Formation of the Myelin Major Dense Line. Sci Rep 7:4974 Available at: http://www.nature.com/articles/s41598-017-05364-3 [Accessed March 4, 2019].

Rasband MN, Peles E (2015) The Nodes of Ranvier: Molecular Assembly and Maintenance. Cold Spring Harb Perspect Biol 8:a020495 Available at: http://www.ncbi.nlm.nih.gov/pubmed/26354894 [Accessed March 3, 2019].

Rasband MN, Peles E, Trimmer JS, Levinson SR, Lux SE, Shrager P (1999) Dependence of nodal sodium channel clustering on paranodal axoglial contact in the developing CNS. J Neurosci.

Rasband MN, Shrager P (2000) Ion channel sequestration in central nervous system axons. J Physiol 525 Pt 1:63-73 Available at: http://www.ncbi.nlm.nih.gov/pubmed/10811725 [Accessed March 5, 2019].

Readhead C, Hood L (1990) The dysmyelinating mouse mutations shiverer (shi) and myelin deficient (shimld). Behav Genet 20:213-234 Available at: http://www.ncbi.nlm.nih.gov/pubmed/1693848 [Accessed March 5, 2019].

Readhead C, Popko B, Takahashi N, Shine HD, Saavedra RA, Sidman RL, Hood L (1987) Expression of a myelin basic protein gene in transgenic shiverer mice: correction of the dysmyelinating phenotype. Cell 48:703-712 Available at: http://www.ncbi.nlm.nih.gov/pubmed/2434242 [Accessed April 16, 2019].

Remahl S, Hildebrand C (1982) Changing relation between onset of myelination and axon diameter range in developing feline white matter. J Neurol Sci 54:33-45 Available at: http://www.ncbi.nlm.nih.gov/pubmed/7077354 [Accessed March 3, 2019].

Reyes SD, Campagnoni AT (2002) Two separate domains in the golli myelin basic proteins are responsible for nuclear targeting and process extension in transfected cells. J Neurosci Res 69:587-596 Available at: http://www.ncbi.nlm.nih.gov/pubmed/12210824 [Accessed March 4, 2019].

Ridsdale RA, Beniac DR, Tompkins TA, Moscarello MA, Harauz G (1997) Threedimensional structure of myelin basic protein. II. Molecular modeling and considerations of predicted structures in multiple sclerosis. J Biol Chem 272:42694275 Available at: http://www.ncbi.nlm.nih.gov/pubmed/9020143 [Accessed March $4,2019]$.

Rinholm JE, Vervaeke K, Tadross MR, Tkachuk AN, Kopek BG, Brown TA, Bergersen LH, Clayton DA (2016) Movement and structure of mitochondria in oligodendrocytes and their myelin sheaths. Glia 64:810-825 Available at: http://www.ncbi.nlm.nih.gov/pubmed/26775288 [Accessed March 6, 2019]. 
Ritchie ME, Phipson B, Wu D, Hu Y, Law CW, Shi W, Smyth GK (2015) limma powers differential expression analyses for RNA-sequencing and microarray studies. Nucleic Acids Res 43:e47-e47 Available at: http://www.ncbi.nlm.nih.gov/pubmed/25605792 [Accessed March 17, 2019].

Rivers LE, Young KM, Rizzi M, Jamen F, Psachoulia K, Wade A, Kessaris N, Richardson WD (2008) PDGFRA/NG2 glia generate myelinating oligodendrocytes and piriform projection neurons in adult mice. Nat Neurosci 11:1392-1401 Available at: http://www.ncbi.nlm.nih.gov/pubmed/18849983 [Accessed March 6, 2019].

Romanelli E, Merkler D, Mezydlo A, Weil M-T, Weber MS, Nikić I, Potz S, Meinl E, Matznick FEH, Kreutzfeldt M, Ghanem A, Conzelmann K-K, Metz I, Brück W, Routh M, Simons M, Bishop D, Misgeld T, Kerschensteiner M (2016) Myelinosome formation represents an early stage of oligodendrocyte damage in multiple sclerosis and its animal model. Nat Commun 7:13275 Available at: http://www.nature.com/articles/ncomms13275 [Accessed February 19, 2019].

Rosenbluth J (1980) Peripheral myelin in the mouse mutant Shiverer. J Comp Neurol 193:729-739 Available at: http://www.ncbi.nlm.nih.gov/pubmed/7440788 [Accessed March 5, 2019].

Rosenbluth J (2009) Multiple functions of the paranodal junction of myelinated nerve fibers. J Neurosci Res 87:3250-3258 Available at: http://www.ncbi.nlm.nih.gov/pubmed/19224642 [Accessed March 3, 2019].

Rosetti CM, Maggio B, Oliveira RG (2008) The self-organization of lipids and proteins of myelin at the membrane interface. Molecular factors underlying the microheterogeneity of domain segregation. Biochim Biophys Acta - Biomembr 1778:1665-1675 Available at: http://www.ncbi.nlm.nih.gov/pubmed/18342620 [Accessed February 27, 2019].

Rushton W a h (1951) A theory of the effects of fibre size in medullated nerve. J Physiol 115:101-122 Available at: http://www.ncbi.nlm.nih.gov/pubmed/14889433 [Accessed February 27, 2019].

Ruskamo S, Yadav RP, Sharma S, Lehtimäki M, Laulumaa S, Aggarwal S, Simons M, Bürck J, Ulrich AS, Juffer AH, Kursula I, Kursula P (2014) Atomic resolution view into the structure-function relationships of the human myelin peripheral membrane protein P2. Acta Crystallogr Sect D Biol Crystallogr 70:165-176 Available at: http://www.ncbi.nlm.nih.gov/pubmed/24419389 [Accessed March 4, 2019].

Saab AS et al. (2016) Oligodendroglial NMDA Receptors Regulate Glucose Import and Axonal Energy Metabolism. Neuron 91:119-132 Available at: http://www.ncbi.nlm.nih.gov/pubmed/27292539 [Accessed March 22, 2019].

Saab AS, Tzvetanova ID, Nave K-A (2013) The role of myelin and oligodendrocytes in axonal energy metabolism. Curr Opin Neurobiol 23:1065-1072 Available at: http://www.ncbi.nlm.nih.gov/pubmed/24094633 [Accessed February 27, 2019].

Sabri MI, Bone AH, Davison AN (1974) Turnover of myelin and other structural proteins in the developing rat brain. Biochem J 142:499-507 Available at:

http://www.ncbi.nlm.nih.gov/pubmed/4142927 [Accessed February 27, 2019].

Saez I, Duran J, Sinadinos C, Beltran A, Yanes O, Tevy MF, Martínez-Pons C, Milán M, Guinovart JJ (2014) Neurons have an active glycogen metabolism that contributes to tolerance to hypoxia. J Cereb Blood Flow Metab 34:945-955 Available at: http://journals.sagepub.com/doi/10.1038/jcbfm.2014.33 [Accessed February 27, 2019]. 
Safaiyan S, Kannaiyan N, Snaidero N, Brioschi S, Biber K, Yona S, Edinger AL, Jung S, Rossner MJ, Simons M (2016) Age-related myelin degradation burdens the clearance function of microglia during aging. Nat Neurosci 19:995-998 Available at: http://www.ncbi.nlm.nih.gov/pubmed/27294511.

Saher G, Brügger B, Lappe-Siefke C, Möbius W, Tozawa R, Wehr MC, Wieland F, Ishibashi S, Nave K-A (2005) High cholesterol level is essential for myelin membrane growth. Nat Neurosci 8:468-475 Available at: http://www.ncbi.nlm.nih.gov/pubmed/15793579 [Accessed February 27, 2019].

Saher G, Quintes S, Mobius W, Wehr MC, Kramer-Albers E-M, Brugger B, Nave K-A (2009) Cholesterol Regulates the Endoplasmic Reticulum Exit of the Major Membrane Protein P0 Required for Peripheral Myelin Compaction. J Neurosci 29:6094-6104 Available at: http://www.ncbi.nlm.nih.gov/pubmed/19439587 [Accessed February 27, 2019].

Sakamoto Y, Kitamura K, Yoshimura K, Nishijima T, Uyemura K (1987) Complete amino acid sequence of PO protein in bovine peripheral nerve myelin. J Biol Chem 262:4208-4214 Available at: http://www.ncbi.nlm.nih.gov/pubmed/2435734 [Accessed March 22, 2019].

Savas JN, Toyama BH, Xu T, Yates JR, Hetzer MW (2012) Extremely Long-Lived Nuclear Pore Proteins in the Rat Brain. Science (80- ) 335:942-942 Available at: http://www.ncbi.nlm.nih.gov/pubmed/22300851 [Accessed February 27, 2019].

Schertel A, Snaidero N, Han H-M, Ruhwedel T, Laue M, Grabenbauer M, Möbius W (2013) Cryo FIB-SEM: Volume imaging of cellular ultrastructure in native frozen specimens. J Struct Biol 184:355-360 Available at: https://linkinghub.elsevier.com/retrieve/pii/S1047847713002657 [Accessed April 9, 2019].

Schieber NL, Machado P, Markert SM, Stigloher C, Schwab Y, Steyer AM (2017) Minimal resin embedding of multicellular specimens for targeted FIB-SEM imaging. Methods Cell Biol.

Schindelin J, Arganda-Carreras I, Frise E, Kaynig V, Longair M, Pietzsch T, Preibisch S, Rueden C, Saalfeld S, Schmid B, Tinevez J-Y, White DJ, Hartenstein V, Eliceiri K, Tomancak P, Cardona A (2012) Fiji: an open-source platform for biological-image analysis. Nat Methods 9:676-682 Available at: http://www.ncbi.nlm.nih.gov/pubmed/22743772 [Accessed March 17, 2019].

Schnaar RL, Collins BE, Wright LP, Kiso M, Tropak MB, Roder JC, Crocker PR (1998) Myelin-associated glycoprotein binding to gangliosides. Structural specificity and functional implications. Ann N Y Acad Sci 845:92-105 Available at: http://www.ncbi.nlm.nih.gov/pubmed/9668345 [Accessed March 6, 2019].

Sinha K, Karimi-Abdolrezaee S, Velumian AA, Fehlings MG (2006) Functional changes in genetically dysmyelinated spinal cord axons of shiverer mice: role of juxtaparanodal Kv1 family K+ channels. J Neurophysiol 95:1683-1695 Available at: http://www.physiology.org/doi/10.1152/jn.00899.2005 [Accessed March 5, 2019].

Snaidero N, Möbius W, Czopka T, Hekking LHP, Mathisen C, Verkleij D, Goebbels S, Edgar J, Merkler D, Lyons DA, Nave K-A, Simons M (2014) Myelin Membrane Wrapping of CNS Axons by PI $(3,4,5)$ P3-Dependent Polarized Growth at the Inner Tongue. Cell 156:277-290 Available at: http://www.ncbi.nlm.nih.gov/pubmed/24439382 [Accessed February 27, 2019].

Snaidero N, Velte C, Myllykoski M, Raasakka A, Ignatev A, Werner HB, Erwig MS, Möbius W, 
Kursula P, Nave K-A, Simons M (2017) Antagonistic Functions of MBP and CNP Establish Cytosolic Channels in CNS Myelin. Cell Rep 18:314-323 Available at: http://www.ncbi.nlm.nih.gov/pubmed/28076777 [Accessed March 4, 2019].

Stoffel W, Boison D, Büssow H (1997) Functional analysis in vivo of the double mutant mouse deficient in both proteolipid protein (PLP) and myelin basic protein (MBP) in the central nervous system. Cell Tissue Res 289:195-206 Available at: http://link.springer.com/10.1007/s004410050866 [Accessed March 5, 2019].

Storey JD, Tibshirani R (2003) Statistical significance for genomewide studies. Proc Natl Acad Sci 100:9440-9445 Available at: http://www.ncbi.nlm.nih.gov/pubmed/12883005 [Accessed March 17, 2019].

Suresh S, Wang C, Nanekar R, Kursula P, Edwardson JM (2010) Myelin Basic Protein and Myelin Protein 2 Act Synergistically to Cause Stacking of Lipid Bilayers. Biochemistry 49:3456-3463 Available at: http://www.ncbi.nlm.nih.gov/pubmed/20334434 [Accessed March 4, 2019].

Susuki K (2013) Node of Ranvier disruption as a cause of neurological diseases. ASN Neuro 5:209-219 Available at: http://www.ncbi.nlm.nih.gov/pubmed/23834220 [Accessed March 3, 2019].

Susuki K, Chang K-J, Zollinger DR, Liu Y, Ogawa Y, Eshed-Eisenbach Y, Dours-Zimmermann MT, Oses-Prieto JA, Burlingame AL, Seidenbecher CI, Zimmermann DR, Oohashi T, Peles E, Rasband MN (2013) Three Mechanisms Assemble Central Nervous System Nodes of Ranvier. Neuron 78:469-482 Available at: https://www.sciencedirect.com/science/article/pii/S0896627313002298?via\%253 Dihub [Accessed March 3, 2019].

Tan CS, Koralnik IJ (2010) Progressive multifocal leukoencephalopathy and other disorders caused by JC virus: clinical features and pathogenesis. Lancet Neurol 9:425-437 Available at: https://www.sciencedirect.com/science/article/pii/S1474442210700405?via\%253 Dihub [Accessed March 7, 2019].

Tanaka R, Iwasaki N, Hayashi M, Nakayama J, Ohto T, Takahashi M, Numano T, Homma K, Hamano K, Sumazaki R (2012) Abnormal brain MRI signal in 18q-syndrome not due to dysmyelination. Brain Dev 34:234-237 Available at: http://www.ncbi.nlm.nih.gov/pubmed/21669507 [Accessed March 5, 2019].

Tao-Cheng JH, Rosenbluth J (1984) Extranodal particle accumulations in the axolemma of myelinated frog optic axons. Brain Res 308:289-300 Available at: http://www.ncbi.nlm.nih.gov/pubmed/6332658 [Accessed March 3, 2019].

Thériault P, Rivest S (2016) Microglia: Senescence Impairs Clearance of Myelin Debris. Curr Biol 26:R772-R775 Available at: https://www.sciencedirect.com/science/article/pii/S0960982216307072\#fig1 [Accessed March 6, 2019].

Torkildsen $\emptyset$, Brunborg LA, Myhr K-M, Bø L (2008) The cuprizone model for demyelination. Acta Neurol Scand 117:72-76 Available at: http://www.ncbi.nlm.nih.gov/pubmed/18439226 [Accessed March 7, 2019].

Torvund-Jensen J, Steengaard J, Askebjerg LB, Kjaer-Sorensen K, Laursen LS (2018) The 3'UTRs of Myelin Basic Protein mRNAs Regulate Transport, Local Translation and Sensitivity to Neuronal Activity in Zebrafish. Front Mol Neurosci 11:185 Available at: http://www.ncbi.nlm.nih.gov/pubmed/29946237. 
Torvund-Jensen J, Steengaard J, Reimer L, Fihl LB, Laursen LS (2014) Transport and translation of MBP mRNA is regulated differently by distinct hnRNP proteins. J Cell Sci 127:1550-1564 Available at: http://www.ncbi.nlm.nih.gov/pubmed/24522184 [Accessed March 4, 2019].

Toyama BH, Savas JN, Park SK, Harris MS, Ingolia NT, Yates JR, Hetzer MW (2013) Identification of Long-Lived Proteins Reveals Exceptional Stability of Essential Cellular Structures. Cell 154:971-982 Available at: http://www.ncbi.nlm.nih.gov/pubmed/23993091 [Accessed February 27, 2019].

Traka M, Arasi K, Avila RL, Podojil JR, Christakos A, Miller SD, Soliven B, Popko B (2010) A genetic mouse model of adult-onset, pervasive central nervous system demyelination with robust remyelination. Brain 133:3017-3029 Available at: http://www.ncbi.nlm.nih.gov/pubmed/20851998 [Accessed March 7, 2019].

Tripathi RB, Jackiewicz M, McKenzie IA, Kougioumtzidou E, Grist M, Richardson WD (2017) Remarkable Stability of Myelinating Oligodendrocytes in Mice. Cell Rep 21:316-323 Available at: http://www.ncbi.nlm.nih.gov/pubmed/29020619 [Accessed March 4, 2019].

Umemori H, Satot S, Yagi T, Aizawal S, Yamamoto T (1994) Initial events of myelination involve Fyn tyrosine kinase signalling. Nature 367:572-576 Available at: http://www.ncbi.nlm.nih.gov/pubmed/7509042 [Accessed March 6, 2019].

Uschkureit T, Sporkel O, Stracke J, Bussow H, Stoffel W (2000) Early onset of axonal degeneration in double (plp-/-mag-/-) and hypomyelinosis in triple (plp-/-mbp-/mag-/-) mutant mice. J Neurosci 20:5225-5233 Available at: http://www.ncbi.nlm.nih.gov/pubmed/10884306 [Accessed March 5, 2019].

van Dieren JM, Simons-Oosterhuis Y, Raatgeep HCR, Lindenbergh-Kortleve DJ, Lambers MEH, van der Woude CJ, Kuipers EJ, Snoek GT, Potman R, Hammad H, Lambrecht BN, Samsom JN, Nieuwenhuis EES (2011) Anti-inflammatory actions of phosphatidylinositol. Eur J Immunol 41:1047-1057 Available at: http://doi.wiley.com/10.1002/eji.201040899 [Accessed April 7, 2019].

van Meer G, Voelker DR, Feigenson GW (2008) Membrane lipids: where they are and how they behave. Nat Rev Mol Cell Biol 9:112-124 Available at: http://www.ncbi.nlm.nih.gov/pubmed/18216768 [Accessed February 27, 2019].

Vanhecke D, Graber W, Studer D (2008) Chapter 9 Close-to-Native Ultrastructural Preservation by High Pressure Freezing. Methods Cell Biol.

Vassall KA, Bamm V V., Harauz G (2015) MyelStones: the executive roles of myelin basic protein in myelin assembly and destabilization in multiple sclerosis. Biochem J 472:17-32 Available at: http://www.ncbi.nlm.nih.gov/pubmed/26518750 [Accessed March 4, 2019].

Virchow R (1854) Über das ausgebreitete Vorkommen einer dem Nervenmark analogen Substanz in den tierischen Geweben. Virchows Arch Pathol Anat 6:562-572.

Visscher M, De Henau S, Wildschut MHE, van Es RM, Dhondt I, Michels H, Kemmeren P, Nollen EA, Braeckman BP, Burgering BMT, Vos HR, Dansen TB (2016) Proteome-wide Changes in Protein Turnover Rates in C. elegans Models of Longevity and Age-Related Disease. Cell Rep 16:3041-3051 Available at: http://www.ncbi.nlm.nih.gov/pubmed/27626671 [Accessed February 27, 2019].

Vogel US, Reynolds R, Thompson RJ, Wilkin GP (1988) Expression of the 2?,3?-cyclic nucleotide 3?-phosphohydrolase gene and immunoreactive protein in 
oligodendrocytes as revealed by in situ hybridization and immunofluorescence. Glia 1:184-190 Available at: http://doi.wiley.com/10.1002/glia.440010303 [Accessed March 6, 2019].

von Bartheld CS, Bahney J, Herculano-Houzel S (2016) The search for true numbers of neurons and glial cells in the human brain: A review of 150 years of cell counting. J Comp Neurol 524:3865-3895 Available at: http://www.ncbi.nlm.nih.gov/pubmed/27187682 [Accessed March 22, 2019].

Wang H, Allen ML, Grigg JJ, Noebels JL, Tempel BL (1995) Hypomyelination alters K+ channel expression in mouse mutants shiverer and Trembler. Neuron 15:1337-1347 Available at: http://www.ncbi.nlm.nih.gov/pubmed/8845157 [Accessed March 5, 2019].

Wang SS-H, Shultz JR, Burish MJ, Harrison KH, Hof PR, Towns LC, Wagers MW, Wyatt KD (2008) Functional trade-offs in white matter axonal scaling. J Neurosci 28:40474056 Available at: http://www.ncbi.nlm.nih.gov/pubmed/18400904.

Waxman SG, Bennett M V (1972) Relative conduction velocities of small myelinated and non-myelinated fibres in the central nervous system. Nat New Biol 238:217-219 Available at: http://www.ncbi.nlm.nih.gov/pubmed/4506206 [Accessed March 3, 2019].

Weil M-T, Möbius W, Winkler A, Ruhwedel T, Wrzos C, Romanelli E, Bennett JL, Enz L, Goebels N, Nave K-A, Kerschensteiner M, Schaeren-Wiemers N, Stadelmann C, Simons M (2016) Loss of Myelin Basic Protein Function Triggers Myelin Breakdown in Models of Demyelinating Diseases. Cell Rep 16:314-322 Available at: http://www.ncbi.nlm.nih.gov/pubmed/27346352 [Accessed March 6, 2019].

Werner HB, Kuhlmann K, Shen S, Uecker M, Schardt A, Dimova K, Orfaniotou F, Dhaunchak A, Brinkmann BG, Mobius W, Guarente L, Casaccia-Bonnefil P, Jahn O, Nave K-A (2007) Proteolipid Protein Is Required for Transport of Sirtuin 2 into CNS Myelin. J Neurosci.

White R, Gonsior C, Krämer-Albers E-M, Stöhr N, Hüttelmaier S, Trotter J (2008) Activation of oligodendroglial Fyn kinase enhances translation of mRNAs transported in hnRNP A2-dependent RNA granules. J Cell Biol 181:579-586 Available at: http://www.ncbi.nlm.nih.gov/pubmed/18490510 [Accessed March 4, 2019].

Windrem MS, Schanz SJ, Guo M, Tian G-F, Washco V, Stanwood N, Rasband M, Roy NS, Nedergaard M, Havton LA, Wang S, Goldman SA (2008) Neonatal chimerization with human glial progenitor cells can both remyelinate and rescue the otherwise lethally hypomyelinated shiverer mouse. Cell Stem Cell 2:553-565 Available at: https://linkinghub.elsevier.com/retrieve/pii/S1934590908001331 [Accessed March 5, 2019].

Wood DD, Ackerley CA, Brand B van den, Zhang L, Raijmakers R, Mastronardi FG, Moscarello MA (2008) Myelin localization of peptidylarginine deiminases 2 and 4: comparison of PAD2 and PAD4 activities. Lab Investig 88:354-364 Available at: http://www.ncbi.nlm.nih.gov/pubmed/18227806 [Accessed April 4, 2019].

Woodruff RH, Franklin RJ (1999) Demyelination and remyelination of the caudal cerebellar peduncle of adult rats following stereotaxic injections of lysolecithin, ethidium bromide, and complement/anti-galactocerebroside: a comparative study. Glia 25:216-228 Available at: http://www.ncbi.nlm.nih.gov/pubmed/9932868 [Accessed April 5, 2019].

Yates MA, Juraska JM (2007) Increases in size and myelination of the rat corpus callosum 
during adulthood are maintained into old age. Brain Res 1142:13-18 Available at: http://www.ncbi.nlm.nih.gov/pubmed/17300760 [Accessed March 4, 2019].

Yeung MSY, Djelloul M, Steiner E, Bernard S, Salehpour M, Possnert G, Brundin L, Frisén J (2019) Dynamics of oligodendrocyte generation in multiple sclerosis. Nature 566:538-542 Available at: http://www.ncbi.nlm.nih.gov/pubmed/30675058 [Accessed February 27, 2019].

Yeung MSY, Zdunek S, Bergmann O, Bernard S, Salehpour M, Alkass K, Perl S, Tisdale J, Possnert G, Brundin L, Druid H, Frisén J (2014) Dynamics of oligodendrocyte generation and myelination in the human brain. Cell 159:766-774 Available at: http://www.ncbi.nlm.nih.gov/pubmed/25417154 [Accessed February 27, 2019].

Yin X, Crawford TO, Griffin JW, Tu P h, Lee VM, Li C, Roder J, Trapp BD (1998) Myelinassociated glycoprotein is a myelin signal that modulates the caliber of myelinated axons. J Neurosci 18:1953-1962 Available at:

http://www.ncbi.nlm.nih.gov/pubmed/9482781 [Accessed March 6, 2019].

Yool DA, Edgar JM, Montague P, Malcolm S (2000) The proteolipid protein gene and myelin disorders in man and animal models. Hum Mol Genet 9:987-992 Available at: http://www.ncbi.nlm.nih.gov/pubmed/10767322 [Accessed March 6, 2019].

Young KM, Psachoulia K, Tripathi RB, Dunn S-J, Cossell L, Attwell D, Tohyama K, Richardson WD (2013) Oligodendrocyte dynamics in the healthy adult CNS: evidence for myelin remodeling. Neuron 77:873-885 Available at:

http://www.pubmedcentral.nih.gov/articlerender.fcgi?artid=3842597\&tool=pmcent rez\&rendertype=abstract.

Yuki N, Hartung H-P (2012) Guillain-Barré Syndrome. N Engl J Med 366:2294-2304 Available at: http://www.ncbi.nlm.nih.gov/pubmed/22694000 [Accessed March 3, 2019].

Zalc B, Goujet D, Colman D (2008) The origin of the myelination program in vertebrates. Curr Biol 18:R511-R512 Available at: http://www.ncbi.nlm.nih.gov/pubmed/18579089 [Accessed February 27, 2019].

Zamvil SS, Mitchell DJ, Moore AC, Kitamura K, Steinman L, Rothbard JB (1986) T-cell epitope of the autoantigen myelin basic protein that induces encephalomyelitis. Nature 324:258-260 Available at: http://www.ncbi.nlm.nih.gov/pubmed/2431317 [Accessed March 5, 2019].

Zhang C, Walker AK, Zand R, Moscarello MA, Yan JM, Andrews PC (2012) Myelin Basic Protein Undergoes a Broader Range of Modifications in Mammals than in Lower Vertebrates. J Proteome Res 11:4791-4802 Available at: http://www.ncbi.nlm.nih.gov/pubmed/22420465 [Accessed March 5, 2019].

Zhang Y, Chen K, Sloan SA, Bennett ML, Scholze AR, O'Keeffe S, Phatnani HP, Guarnieri P, Caneda C, Ruderisch N, Deng S, Liddelow SA, Zhang C, Daneman R, Maniatis T, Barres BA, Wu JQ (2014) An RNA-Sequencing Transcriptome and Splicing Database of Glia, Neurons, and Vascular Cells of the Cerebral Cortex. J Neurosci 34:11929-11947 Available at: http://www.ncbi.nlm.nih.gov/pubmed/25186741 [Accessed March 6, 2019].

Zhou T et al. (2019) Microvascular endothelial cells engulf myelin debris and promote macrophage recruitment and fibrosis after neural injury. Nat Neurosci 22:421-435 Available at: http://www.nature.com/articles/s41593-018-0324-9 [Accessed February 27, 2019]. 
Zuchero JB, Fu M, Sloan SA, Ibrahim A, Olson A, Zaremba A, Dugas JC, Wienbar S, Caprariello AV, Kantor C, Leonoudakis D, Lariosa-Willingham K, Kronenberg G, Gertz K, Soderling SH, Miller RH, Barres BA, Barres BA (2015) CNS Myelin Wrapping Is

Driven by Actin Disassembly. Dev Cell 34:152-167 Available at: http://www.ncbi.nlm.nih.gov/pubmed/26166300 [Accessed February 27, 2019]. 
Curriculum Vitae 